\title{
Advanced Hot Gas Filter Development
}

Final Report

September 29, 1994 to September 30, 2000

E. S. Connolly

G. D. Forsythe

September 30, 2000

Work Performed Under Contract: DE-AC21-94MC31214

DOE/NETL Project Manager: T. J. McMahon

For

U. S. Department of Energy

Office of Fossil Energy

National Energy Technology Laboratory

Morgantown, West Virginia

Prepared by

Honeywell Advanced Composites Inc.

(formerly AlliedSignal Composites Inc.)

(formerly DuPont Lanxide Composites Inc.)

400 Bellevue Road

Newark, DE 19713 


\section{DISCLAIMER}

This report was prepared as an account of work sponsored by the United States Government. Neither the United States nor the United States Department of Energy, nor any of their employees, makes any warranty, expressed or implied, or assumes any legal liability or responsibility for the accuracy, completeness, or usefulness of any information, apparatus, product, or process disclosed, or represents that its use would not infringe privately owned rights. Reference herein to any specific commercial product, process, or service by trade name, mark, manufacturer, or otherwise, does not necessarily constitute or imply its endorsement, recommendation, or favoring by the United States Government or any agency thereof. The views and opinions of authors expressed herein do not necessarily state or reflect those of the United States Government or any agency thereof.

\section{PATENT STATUS}

In July 1997, a patent was filed which covered the development of an improved surface filtration membrane for hot gas filters. This patent was filed as a "continuation-in-part" to the original Hot Gas Filter Patent $(5,460,637)$ owned by DuPont Lanxide Composites Inc. In September 1997, the United States Department of Energy granted DuPont Lanxide Composites Inc. a waiver request for the subject invention, DOE Docket No. S-88,782. This patent was granted and issued May 11, 1999 as U.S. Patent 5,902,363. A copy has been supplied as Appendix 1 Additional foreign applications were filled in Australia, China, India, Japan, South Korea, Singapore, and the European Patent Convention.

\section{TECHNICAL STATUS}

This technical report is being transmitted in advance of DOE review and no further dissemination or publication shall be made of the report without prior approval of the DOE Project/Program Manager. 
CONTRACTOR'S NOTE: Identity of Contracted Parties

Contract \#DE-AC21-94MC31214 was awarded to DuPont Lanxide Composites Inc.(DLC) in September 1994. In August 1998, DuPont Lanxide Composites Inc. was acquired by AlliedSignal Inc., and renamed AlliedSignal Composites Inc. (ACI). In December 1999, AlliedSignal Inc. merged with the Honeywell Company to form Honeywell International. As a result of this merger, AlliedSignal Composites Inc. was renamed Honeywell Advanced Composites Inc.(HACI). Novations and "change of name agreements" were performed by DCMC.

Throughout this report, the all three versions of the contractor's name are used, depending on the period of time in which the work was performed. Likewise, the contracting agency is referenced to as the Morgantown Energy Technology Center (METC), the Federal Energy Technology Center (FETC) or the National Energy Technology Laboratory (NETL) depending on when the original material was documented. Furthermore, our subcontractor is referred to as the Westinghouse Science and Technology Center (W-STC) or Siemens Westinghouse Power Corporation (SWPC), depending on whether the original reference was made before the Siemens acquisition of this Westinghouse business. 


\section{TABLE OF CONTENTS}

1. $\quad \mathrm{EXECUTIVE} S \mathrm{~S} M \mathrm{MARY}$ -

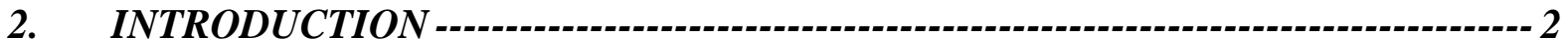

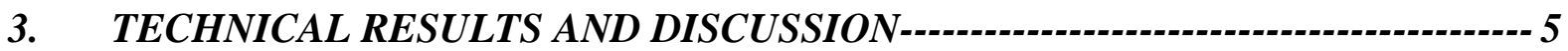

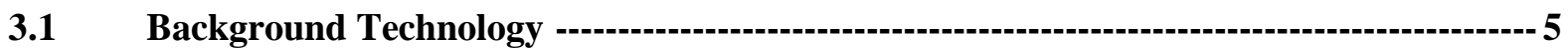

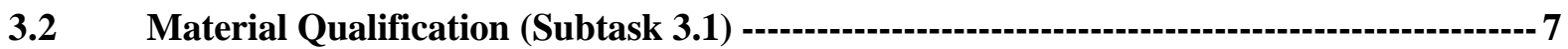

3.2.1 Improving the Surface Membrane---

3.2.2 Development of a Dual Membrane Candle Filter -- 10

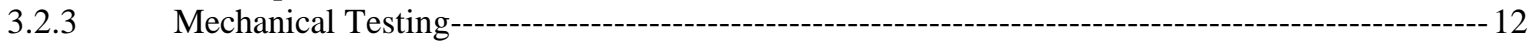

3.2.4 Strengthened Flanges-------- 15

3.2.5 Filtration and Permeability Testing ------------------------------------------- 18

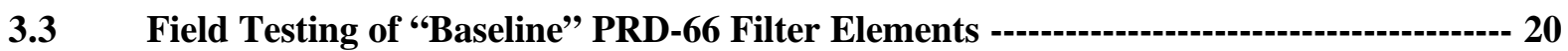

3.3.1 Tidd Test Segment 4 -- 20

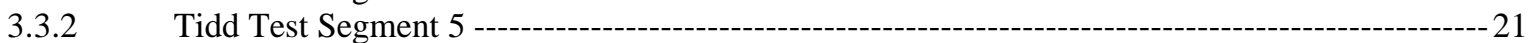

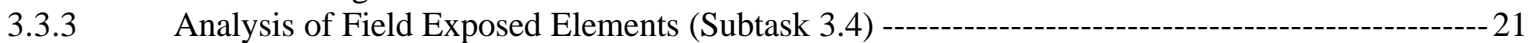

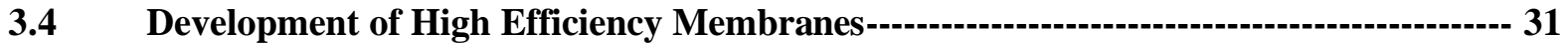

3.4.1 Membrane Development for the Outside Diameter (OD) Surface (Subtask 3.1)-----------31

3.4.2 Membrane Development for the Inside Diameter (ID) Surface (Subtask 3.1.1)----------------- 35

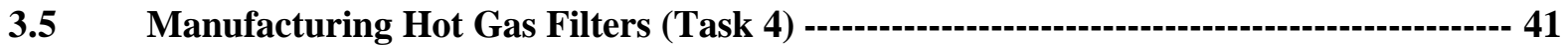

3.5.1 Raw Materials Plan (Subtask 4.1) --41

3.5.2 Process Instrumentation (Subtask 4.2) -

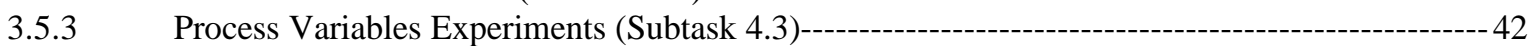

3.5.4 Process Capability Demonstration (Subtask 4.4) -

3.5.5 Equipment Analysis and Improvement (Subtask 4.5)--

3.5.6 Redesign of Prototype Winding Equipment (Subtask 4.7) ---

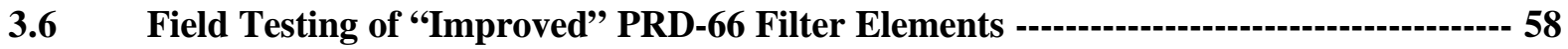

3.6.1 High Temperature High Pressure (HTHP) Testing at W-STC---

3.6.2 PCFBC Exposure at Karhula-- 63

3.7 Manufacturing 50 Filters (Task 5) -------------------------------------------------------- 77

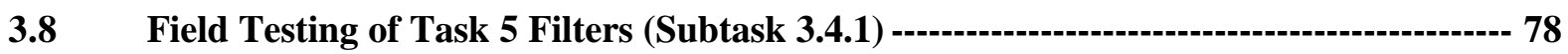

3.8.1 PSDF Exposure History-- 78

3.8.2 Failure Characterization and Investigation -- 80

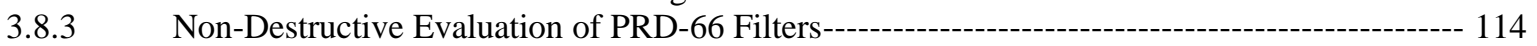

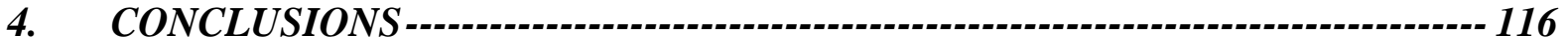

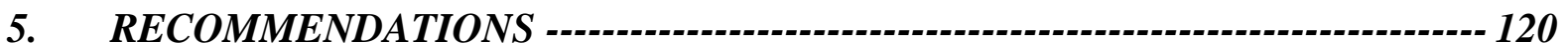

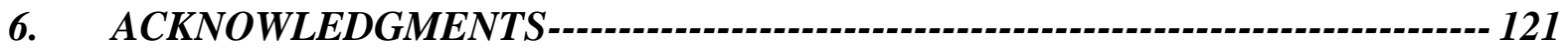

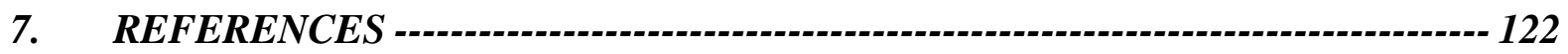




\section{LIST OF FIGURES}

Figure 1 - Schematic of the basic process for winding PRD-66 cylindrical structures. ---------------------- 6

Figure 2 - Schematic of the PRD-66 membrane winding process. ----------- 6

Figure 3 - Phase diagram including PRD-66 composition.---

Figure 4 - Impact of three PRD-66 variables on backpressure $(\Delta P)$, where " $N$ " is the number of samples. ----- 9

Figure 5 - Impact of inside diameter membrane on backpressure $(\Delta P)$, where " $N$ " is the number of samples. -11

Figure 6 - Typical Load Displacement Curve for PRD-66 filter segment. ---------------------------- 12

Figure 7 - O-rings AFTER (left) and BEFORE (right) diametrical compression testing. --------------------- 13

Figure 8 - Weibull Analysis of PRD-66 candle filter segment. --

Figure 9 - Strain rate dependence of o-ring crushing test.--

Figure 10 - O-ring diametrical compressive strength versus ring weight. --_- 16

Figure 11 - O-ring diametrical compressive strength vs. amount \& viscosity of infiltrate ---

Figure 12 - Load Displacement Curve for infiltrated PRD-66 filter segment. ---------------------------- 17

Figure 13 - W-STC Room Temperature Gas Flow Resistance measurements of 1.5-meter PRD-66 filter elements with various membranes. ${ }^{12}$-.---

Figure 14 - "Divots" in PRD-66 filter tested in Tidd Test Segment 5. -

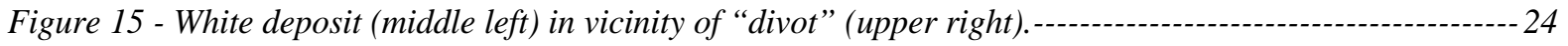

Figure 16 - Unit-cell size of magnesium sulfate versus state of hydration. ---

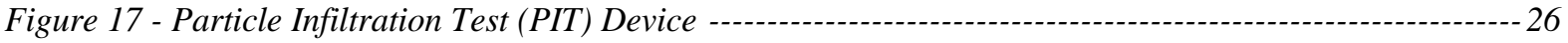

Figure 18 - PIT-exposed sample viewed in transmitted light. -

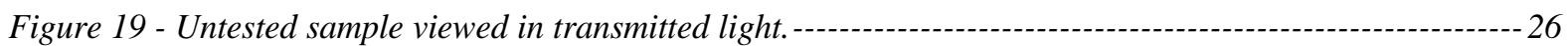

Figure 20 - Exaggerated illustration of a PRD-66 delamination ---

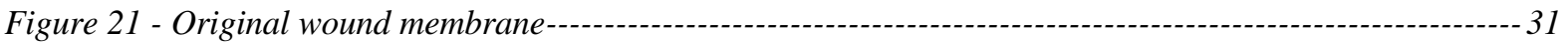

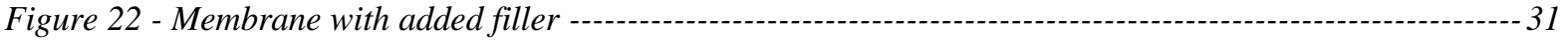

Figure 23 - Modified membrane with PIT rating of "10". -

Figure 24 - Hole in membrane, undetectable under direct light.--

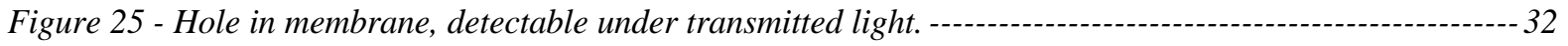

Figure 26 - Hole in membrane after 25 PIT cycles, viewed in transmitted light. -

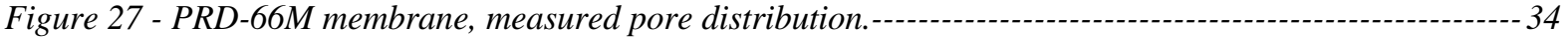

Figure 28 - PRD-66M flow resistance for 1.5-meter candles. --

Figure 29 - PRD-66C membrane, measured pore distribution. ---

Figure 30 - PRD-66C flow resistance for 1.5-meter candles. ---

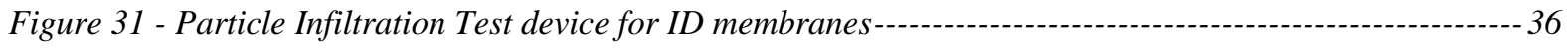

Figure 32 - ID P.I.T. evaluation of original "hoop-only dual membrane" filter (1995)----------------------- 36

Figure 33 - Standard filter support scheme during membrane-filler application. ------------------- 37

Figure 34 - Cantilevered filter support for membrane-filler application. --o

Figure 35 - ID P.I.T. evaluation of "slip-cast ID membrane" filter (no membrane on OD) --------------- 39

Figure 36 Device for removing excess membrane from the inside surface of a filter. ------------------------- 40

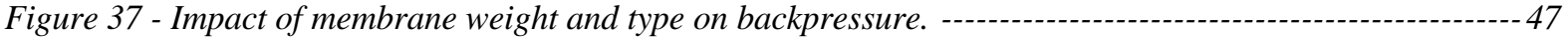


Figure 38 - PRD-66 Candle Filter dimensions --

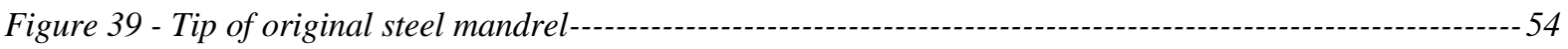

Figure 40 - Original hoop membrane ----------------------------- 57

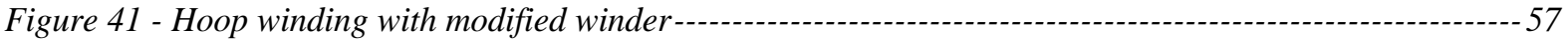

Figure 42 - PRD-66C - Room temperature gas flow resistance ${ }^{12}$--

Figure 43 - PRD-66M - Room temperature gas flow resistance ${ }^{12}$

Figure 44 - Gas flow resistance of as-manufactured and HTHP-exposed PRD-66M elements ${ }^{12}$------------- 60

Figure 45 - Gas flow resistance of as-manufactured and HTHP-exposed PRD-66C elements ${ }^{12}$--------------- 60

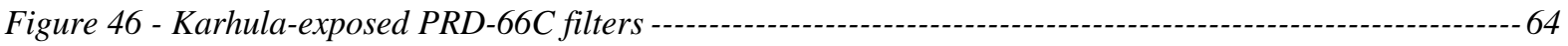

Figure 47 - Outside diameter of Karhula-exposed element before ash removal ---

Figure 48 - Inside diameter of Karhula-exposed element before ash removal --

Figure 49 - Differential pressure of Karhula filters measured by Foster Wheeler --.---_--- 66

Figure 50 - Wall interior of Karhula-exposed candle \#577------------------- 67

Figure 51 - Close-up of \#577 - OD surface and 1-2mm below --------------- 67

Figure 52 - 300X - UNEXPOSED candle surface--

Figure 53 - 300X - EXPOSED candle surface -

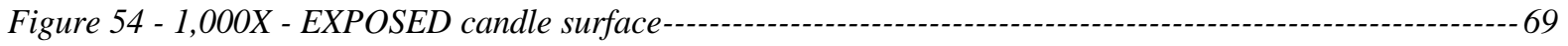

Figure 55 - UNEXPOSED CANDLE, cross-section of membrane filler (300X) --

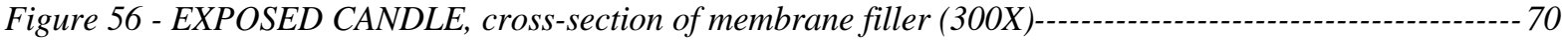

Figure 57 - 25X, fast-fracture - UNEXPOSED CANDLE, interior of support wall -

Figure 58 - 25X, fresh-fracture - EXPOSED CANDLE, interior of support wall -- 71

Figure 59 - 50X, fast-fracture - UNEXPOSED CANDLE, interior of support wall ----------------- 71

Figure 60 - 50X, fast-fracture - EXPOSED CANDLE, interior of wall support---_- 72

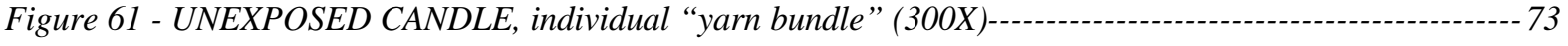

Figure 62 - EXPOSED CANDLE, individual “yarn bundle” (300X)- 73

Figure 63 - UNEXPOSED CANDLE, individual "yarn bundle” (1,000X) - 74

Figure 64 - EXPOSED CANDLE, individual “yarn bundle” $(1,000 X)$-------- 74

Figure 65 - Aftermath of Oct'98 thermal event in the lower plenum at the PSDF. ${ }^{13}$--

Figure 66 - C740 (T12), 18-19” from open end----

Figure 67 - C749 (T33), 12” from open end --

Figure 68 - Spalled area of Filter \#C740, 18"from open end, no other similar damage --

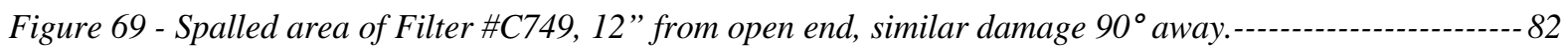

Figure 70 - Damage resulting from impact testing of Filter C633, Alumina Lot\#1, 1360 exposure hours ------ 84

Figure 71 - Damage resulting from impact testing of Filter C740, Alumina Lot\#2, 636 exposure hours-------- 84

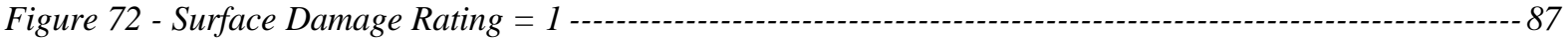

Figure 73 - Surface Damage Rating = 4 --

Figure 74 - C756 (T-31) broke during reinstallation --- 89

Figure 75 - $C 750$ (B-49) with fine crack at base of tapered region of flange ---------------------------- 89 
Figure 76 - Description of flange contours as determined by Optical Comparator

Figure 77 - PRD-66 Flange Measurement Technique --- 92

Figure 78 - Standard-shape PRD-66 flange -- 93

Figure 79 - Modified PRD-66 flange using manual techniques -- 93

Figure 80 - C750, similar to C731 and C752 - 94

Figure 81 - C749 (T33), flange end---10--- 94

Figure 82 - Broken end of Filter C752, with deep chip 11/4” below the break-- 95

Figure 83 - C745 (B-54)-- 96

Figure 84 - C633, broke removing filter-nut-- 96

Figure 85 - Box Plot of o-ring compressive strength for as-manufactured filters. --------- 97

Figure 86 - Average o-ring compressive strength for as-manufactured filters, ----------------------------- 98

Figure 87 - Box Plot of o-ring compressive data grouped by alumina lot number.------ 99

Figure 88 - X-ray Diffraction Comparison of Filter \#638 (Alumina Lot \#1) and Filter \#713 (Alumina Lot \#2) shows no differences in crystalline phases. ----

Figure 89 - Cutting plan for tensile coupons from PRD-66C “as-manufactured filters”. -----------------102

Figure 90 - Filter cross-section depicting geometry of tensile coupons. -- 102

Figure 91 - Axial Tension stress/strain curves for unexposed filters made with Alumina Lot\#1----103

Figure 92 - Axial Tension stress/strain curves for unexposed filters made with Alumina Lot\#2---103

Figure 93 - Microstructure of PRD-66C Filter \#C567, 500X -- 104

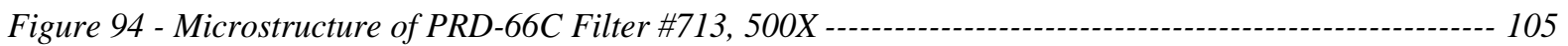

Figure 95 - Microstructure of PRD-66C Filter \#C752, 500X -- 106

Figure 96 - Fresh-fracture SEM of PRD-66C Filter \#C567 (high strength) at 500X ----107

Figure 97 - Fresh-fracture SEM of PRD-66C Filter \#C713 (low strength) at 500X ---107

Figure 98 - Fresh-fracture SEM of \#C567 in high-alumina region at 1,000X --- 108

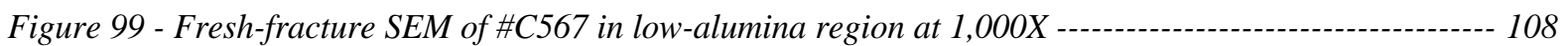

Figure 100 - Alumina content versus average o-ring diametrical compressive strength for as-manufactured PRD-66 filters made with Alumina Lot \#2 -- 109

Figure 101 - PRD-66 4-point flex coupons - cross-section -- 111

Figure 102 - PRD-66 4-point flex coupons - side-cut view -- 11

Figure 103 - 4-Point Flex Test set-up of PRD-66 coupons from regions within 4" of the flange---------111

Figure 104 - Maximum 4-Point Load for the Flex Testing of PRD-66 filters in three different regions: infiltrated zone, transition zone, and uninfiltrated zone--- 112

Figure 105 - Load-Displacement Curves for 4-point flex tests in the "transition (edge) zones". ---113

Figure 106 - Preliminary NDE results on PRD-66 hot gas filters, comparing ANL's Stress Wave Value to the oring diametrical compressive strength of the same filter. ---

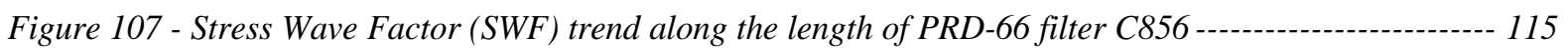




\section{LIST OF TABLES}

Table 1 - Impact of "intermediate" matrix ratio on backpressure $(\Delta P)$ at $5 \mathrm{scfm}-10$

Table 2 - Impact of dual membranes on backpressure $(\Delta P)$ at 5 scfm. -

Table 3 - Average o-ring diametrical compressive strength ( $n$ " is the number of samples). --------14

Table 4 - Comparison of test conditions in Tidd Test Segments 4 and 5. ------------- 22

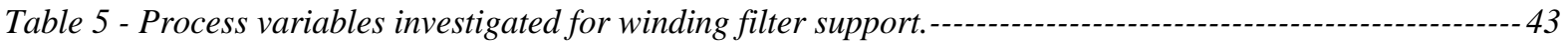

Table 6 - Observed impact of process changes on support winding. ---

Table 7 - Impact of grit-size and binder content on backpressure. ---

Table 8 - Impact of binder content on backpressure of PRD-66C. -

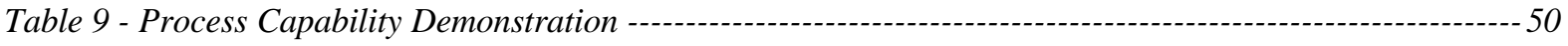

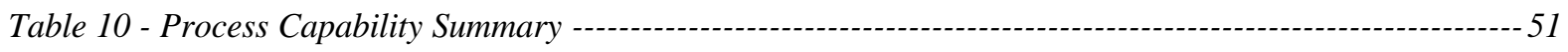

Table 11 - W-STC Room temperature and process strength of PRD-66 elements ${ }^{12}$

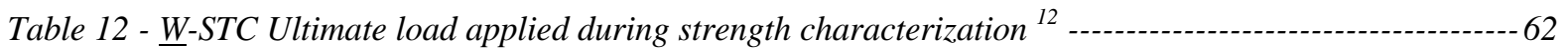

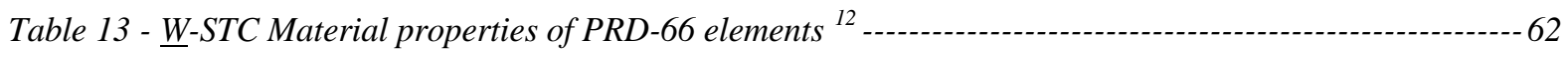

Table 14 - DLC Diametrical compression testing of HTHP-exposed \& unexposed candles ------------------- 62

Table 15 - Karhula PCFBC test conditions ---

Table 16 - O-ring diametrical compressive testing of Karhula-exposed \& unexposed candles ----------- 75

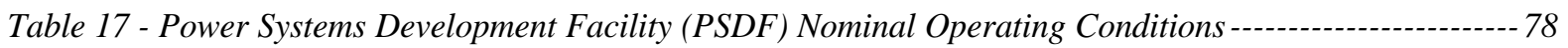

Table 18 - Damage Resulting from Impact Testing of Exposed Filters---

Table 19 - Design of Impact Damage Experiment --------------------- 85

Table 20 - Effect of Alumina Content and Lot on Surface Damage Rating ---

Table 21 - Effect of Alumina Content and Lot on Depth of Damage--

Table 22 - Nominal Crystalline Phase Composition of PRD-66 --- 100

Table 23 - O-ring Strength of As-Manufactured PRD-66 Filter -- 101 


\section{EXECUTIVE SUMMARY}

DuPont Lanxide Composites, Inc. undertook a sixty-month program, under DOE Contract DEAC21-94MC31214, in order to develop hot gas candle filters from a patented material technology know as PRD-66. The goal of this program was to extend the development of this material as a filter element and fully assess the capability of this technology to meet the needs of Pressurized Fluidized Bed Combustion (PFBC) and Integrated Gasification Combined Cycle (IGCC) power generation systems at commercial scale.

The principal objective of Task 3 was to build on the initial PRD-66 filter development, optimize its structure, and evaluate basic material properties relevant to the hot gas filter application. Initially, this consisted of an evaluation of an advanced filament-wound core structure that had been designed to produce an effective bulk filter underneath the barrier filter formed by the outer membrane. The basic material properties to be evaluated (as established by the DOE/METC materials working group) would include mechanical, thermal, and fracture toughness parameters for both new and used material, for the purpose of building a material database consistent with what is being done for the alternative candle filter systems. Task 3 was later expanded to include analysis of PRD-66 candle filters, which had been exposed to actual PFBC conditions, development of an improved membrane, and installation of equipment necessary for the processing of a modified composition.

Task 4 would address essential technical issues involving the scale-up of PRD-66 candle filter manufacturing from prototype production to commercial scale manufacturing. The focus would be on capacity (as it affects the ability to deliver commercial order quantities), process specification (as it affects yields, quality, and costs), and manufacturing systems (e.g. QA/QC, materials handling, parts flow, and cost data acquisition). Any filters fabricated during this task would be used for product qualification tests being conducted by Westinghouse at Foster-Wheeler's Pressurized Circulating Fluidized Bed (PCFBC) test facility in Karhula, Finland.

Task 5 was designed to demonstrate the improvements implemented in Task 4 by fabricating fifty 1.5-meter hot gas filters. These filters were to be made available for DOE-sponsored field trials at the Power Systems Development Facility (PSDF), operated by Southern Company Services in Wilsonville, Alabama. 


\section{INTRODUCTION}

Advanced, coal-based power plants will require durable and reliable hot gas filtration systems to remove particulate contaminants from the gas streams to protect downstream components such as turbine blades from erosion damage. It is expected that the filter elements in these systems will have to be made of ceramic materials able to withstand goal service temperatures of $1600^{\circ} \mathrm{F}$ or higher. Prior to 1994, demonstration projects and pilot plant tests indicated that the current generation of ceramic hot gas filters (cross-flow and candle configurations) fail prematurely. Two of the most promising materials that had been extensively evaluated were clay-bonded silicon carbide ${ }^{1,2}$ and alumina-mullite porous monoliths. These candidates, however, were been found to suffer progressive thermal shock/fatigue damage, because of rapid cooling/heating cycles. Such temperature changes occur regularly when the hot filters are back-pulsed with cooler gas to clean them, or in process upset conditions, where even larger gas temperature changes may occur quickly and unpredictably. ${ }^{9}$ In addition, the clay-bonded silicon carbide materials are susceptible to chemical attack of the glassy binder phase that holds the $\mathrm{SiC}$ particles together, resulting in softening, strength loss, creep, and eventual failure. ${ }^{1}$

To address these issues, Du Pont Lanxide Composites (DLC) developed a unique and innovative new candle filter made from a ceramic material called PRD-66. This material, is an extensively micro-cracked structure comprising a mixture of crystalline oxide phases (primarily mullite, cordierite, and corundum). It combines the high chemical stability inherent in the oxide ceramics with a thermal shock resistance typically found only in state-of-the art, fiber-reinforced, ceramic matrix composites. The highly micro-cracked structure provides an effective mechanism for stopping crack propagation through the material. ${ }^{7}$

An additional attribute of PRD-66 ceramic structures is that unlike many whisker-reinforced ceramic composites, they contain no respirable ceramic fibers. This makes handling, installation, and removal of the filters a simpler task, requiring no special protective equipment or record keeping, necessary to comply with the increasing health concerns and likely regulations governing personnel exposure to non-asbestos respirable fibers (NARFS). ${ }^{7}$

Based on its low-cost ingredients and relatively simple manufacturing process, commercial quantity costs of PRD-66 hot gas filters are expected to be fully competitive with the clay-bonded $\mathrm{SiC}$ and alumina-mullite monolithic filters that have been involved in earlier demonstration programs.

Prototype PRD-66 candle filters are comprised of a cleanable porous membrane structure over a core that is inherently a bulk filter. Should the membrane become locally damaged by an impact 
e.g., during installation. The exposed core structure would continue to filter out particulates, until it eventually "blinds", effectively healing the damaged section while the rest of the filter continues to perform as designed.

Early development activity included a preliminary material characterization and the demonstration of acceptable permeability and dust retention properties. One-meter working prototypes were manufactured and tested in cooperation with Westinghouse Science and Technology Center. ${ }^{2}$ Testing included short-term, high temperature, high pressure exposure to simulated Pressurized Fluidized Bed Combustion conditions under steady state and thermal transients (accelerated pulse cleaning and turbine trip simulations). Although limited, this testing was sufficiently encouraging to stimulate production of 1.5-meter prototypes with a flange configuration that was designed to allow retrofit in existing demonstration units.

Based on the initial development successes of PRD-66 hot gas candle filter prototypes, ${ }^{2}$ the goal of this program was to extend the development of PRD-66 candle filters and fully assess the capability of this technology to meet the needs of PFBC and IGCC power generation systems at commercial scale. The work emphasized optimizing the filter body and flange configurations, demonstrating goal mechanical durability in qualification testing under normal and "upset" operating conditions, and defining and addressing the key issues involved in manufacturing PRD-66 hot gas filters at commercial scale.

The scientific and engineering rationale for developing PRD-66 as a hot gas filtration media was supported by the following evidence:

- The chemical stability of these oxides in coal combustion environments is well known. ${ }^{3}$

- PRD-66 has an extended use temperature of over 1200 degrees Celsius (2200 $\mathrm{F})$. This service temperature significantly exceeds the goals of current coal combustion programs, and keeps the way open to higher temperature higher thermodynamic efficiency combustion processes in the future. ${ }^{7,8}$

- Microcracked structures such as this, in addition to being inherently porous filtering structures, are very effective at preventing crack propagation. Because of this microstructure, the thermal shock resistance of PRD-66 is outstanding. In catalyst support applications PRD-66 was subjected to multiple thermal downshocks (theoretically exceeding $10,000^{\circ} \mathrm{C} / \mathrm{second}$ ) in turbine trip simulations without damage. ${ }^{7,8}$

- By using highly developed textile and composite forming technologies, the precise location of each yarn can be controlled and structures fabricated with independent control of gas paths, porosities, and backpressure. This allows for the creation of filters having a thin, low pressure drop surface 
barrier, backed up by a bulk-filter core that acts as a secondary, backup filter to protect the turbine, should the filter surface be mechanically damaged during installation or operation. ${ }^{7,8}$

- The manufacturing process is simple, well controlled, and readily scaleable.

- The raw materials (fiberglass yarn and alumina) are inexpensive and readily available. This offers a route to advanced filters that will be price competitive with the current generation of hot gas filters. ${ }^{7,8}$

- Capacity can be readily expanded with minimal new investment. This offers a clear path to scaleup without requiring the industry to support large capital investments or wait a long time to evaluate or adopt the technology on a commercial scale. 


\section{TECHNICAL RESULTS AND DISCUSSION}

\subsection{Background Technology}

PRD-66 all-oxide ceramic materials were invented and patented by DuPont and assigned to Du Pont Lanxide Composites (DLC), a joint venture company owned by E. I du Pont de Nemours, Inc. and Lanxide, Inc. A fiberglass yarn is coated with a suspension of alumina in water, and placed by high precision fiber handling techniques, in this case, filament winding, into the net shape of the filter. This preform is allowed to dry, then fired through a proprietary firing cycle. In this firing process, the silica and magnesia in the fiberglass react with the alumina in the slurry to form mullite and cordierite. The surface of the material is unreacted alumina. It should be noted that the fiberglass is consumed in this chemical reaction, and the resulting product is not fiber reinforced. ${ }^{7}$

For several years prior to the initiation of this project, DuPont, DLC, and Westinghouse Electric Corporation cooperated in the fabrication and early testing of hot gas candle filters based on the PRD-66 technology. The result of that collaboration will, hereafter in this report, be referred to as the "baseline" PRD-66 Candle Filter.

The raw materials required to produce a "baseline" PRD-66 Candle Filter are fiberglass yarn (S-2 type, produced by Owens Corning), calcined alumina power (A-17, produced by Alcoa), fumed alumina powder (produced by Degussa), and deionized water.

The flange, body and membrane portions of the PRD-66 Candle Filter are all produced by coating the fiberglass yarn with a precise amount of alumina slurry and winding the coated filament onto a spinning mandrel. ${ }^{7}$

The first step in producing a PRD-66 Candle Filter is the fabrication of the flange segment. This operation is performed, as shown in Figure 1, on a small winder (max. unit length $=6$ inches). The slurry-coated yarn is wound onto a $46 \mathrm{~mm}$ diameter mandrel with a removable plastic sleeve. When the cylindrical structure is $60 \mathrm{~mm}$ in diameter, the winding is stopped. The "integral flange" and the plastic sleeve are then removed from the flange mandrel, and slid onto the filter mandrel, which had been previously covered with a plastic sleeve along its entire length. The integral flange is positioned at the appropriate position from the tip end of the mandrel. 


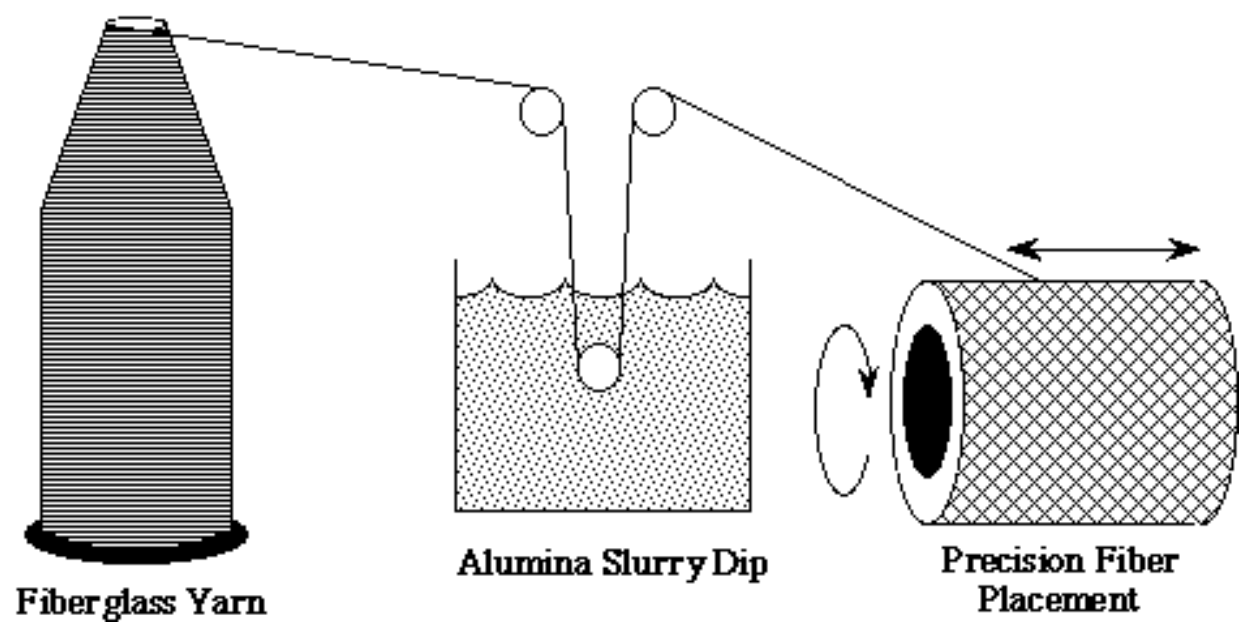

Figure 1 - Schematic of the basic process for winding PRD-66 cylindrical structures.

The winding of the filter support is then performed, as shown in Figure 1, on a winder capable of producing 65-inch long cylindrical structures. As the slurry-coated yarn is applied to the mandrel, it encases the integral flange. Winding proceeds until the outside diameter of the tube is $60 \mathrm{~mm}$, yielding a flange diameter of $74 \mathrm{~mm}$.

The winding of the membrane yarn is then performed, as shown Figure 2, on a winder which has been specially designed for laying down the yarn at approximately $90^{\circ}$ to the axis of the mandrel. The winding begins at the tip end of the candle support structure; each successive "hoop" is laid down immediately adjacent to the previous one. Winding proceeds along the straight portion of the filter, then over the flange portion of the filter, creating a single layer of membrane yarn.

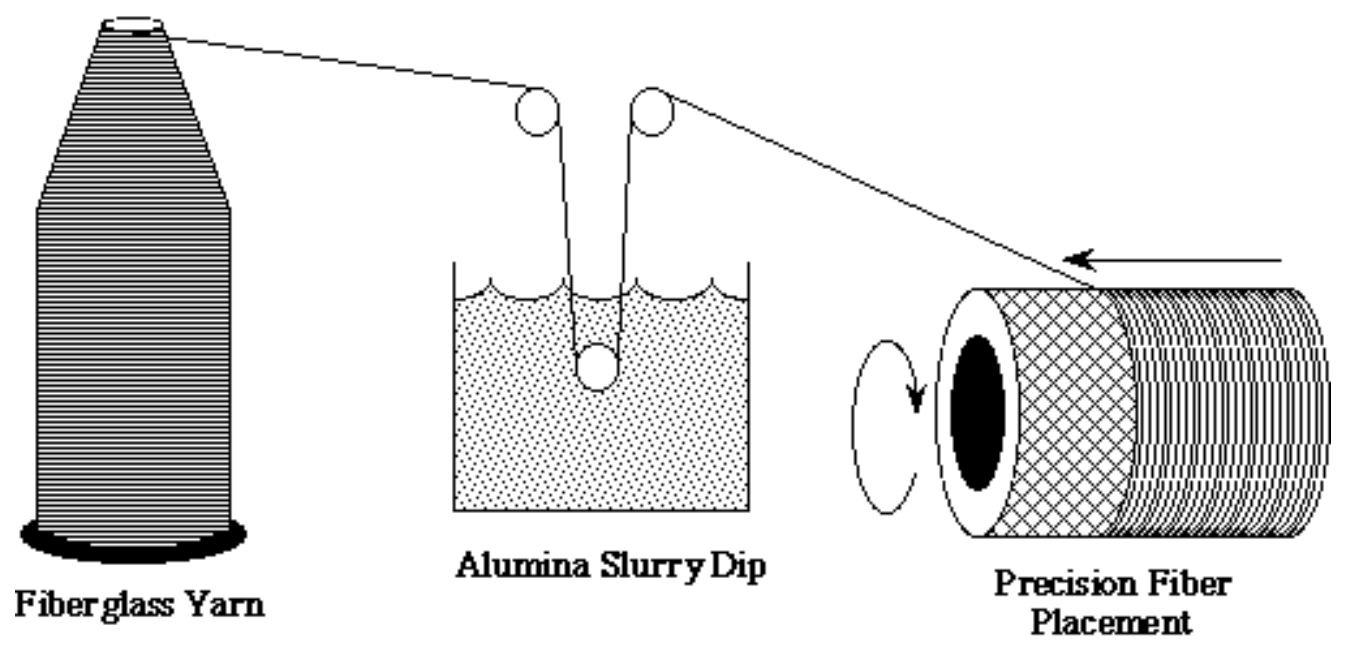

Figure 2 - Schematic of the PRD-66 membrane winding process.

The filter is then dried overnight on the mandrel, cut to length, and removed from the mandrel. A paste-like substance (comprised of the same raw materials as the filter itself) is then used to fill the 
hole left in the tip of the candle by the mandrel. The filter is then heated to approximately $1400^{\circ} \mathrm{C}$ in air. During this firing, the alumina coating reacts with the silica, magnesia, and alumina in the glass yarn to form a layered, microcracked structure comprising primarily cordierite, mullite, and corundum.

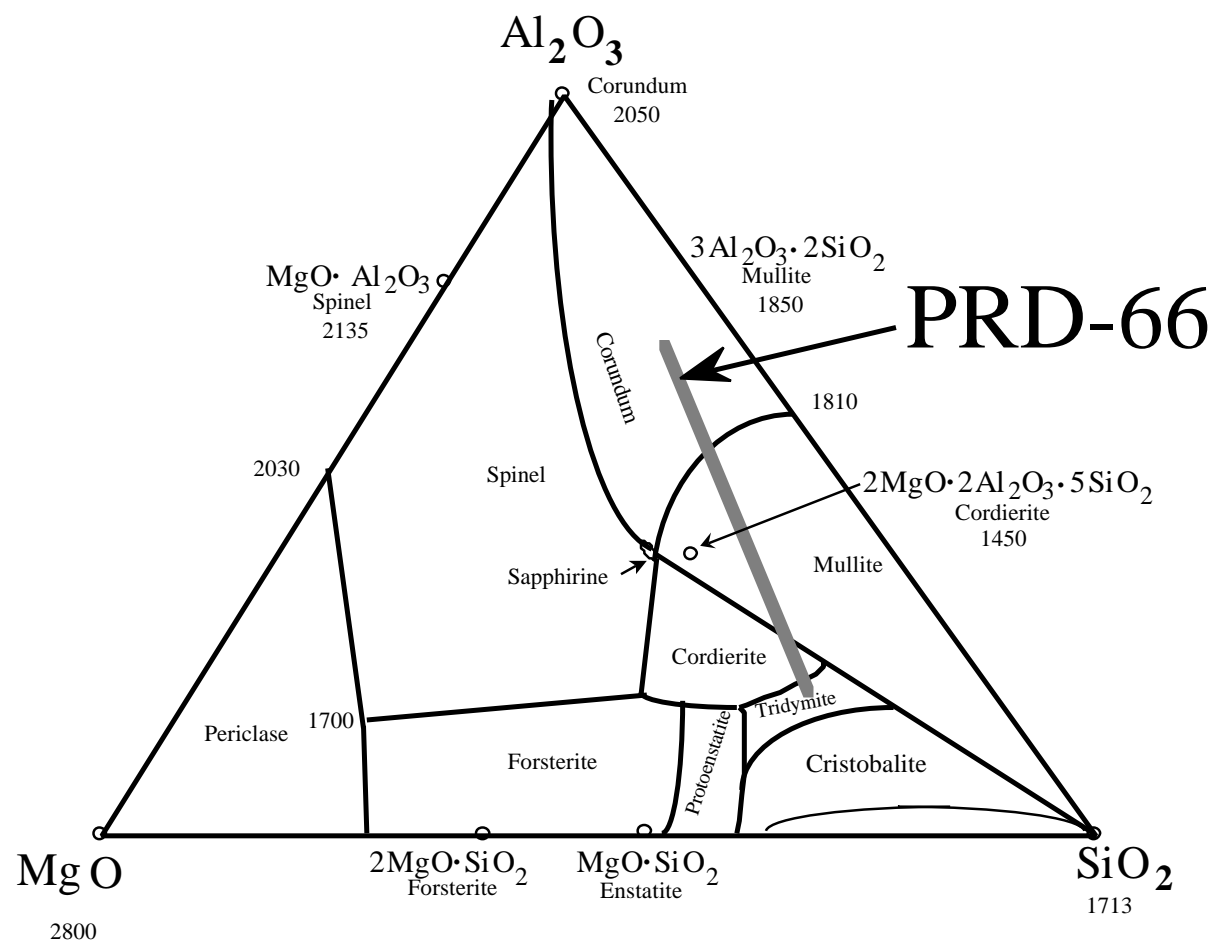

Figure 3 - Phase diagram including PRD-66 composition.

This "baseline" PRD-66 filter was successfully tested in Ohio Power's Tidd PFBC facility in the late summer through early fall of $1994 .^{2}$

\subsection{Material Qualification (Subtask 3.1)}

In Subtask 3.1, attempts were made to improve the design of the baseline candle filter. The design improvements sought included:

1. improved surface filtration membrane for reduced pressure drop

2. a "dual membrane" filter (with membranes on the inside and outside surfaces) having acceptable backpressure

3. increased strength of the flange region

Full size candle filters, which incorporated these attempts at design changes, were fabricated. These filters were then tested by our subcontractor, Westinghouse Science and Technology Center, to assure that the improved filters still met the fundamental requirements of acceptable permeability and 
filtration efficiency. After this testing, a decision on which improvements were successful were made, and full sized candle filters incorporating the selected improvements were produced for testing in subsequent tasks.

Also in Subtask 3.1, mechanical property tests suitable for monitoring progress toward stronger filters, and ultimately for process control, were surveyed. After choosing the best test, the mechanical properties of the baseline filter were determined and an evaluation of strength improvements was performed.

\subsubsection{Improving the Surface Membrane}

In attempting to improve the surface filtration membrane on the PRD-66 candle filter, while retaining good filtration characteristics, two properties had to be considered. Firstly, a lower backpressure membrane is desirable. Secondly, a membrane that will release the ashcake more easily is desirable. In the grossest qualitative sense, a smooth appearance on the surface of the filter is thought to be important to good cake release, and can be assessed visually. In the absence of an effective quantitative test, DLC attempted to maintain the same degree of smoothness in the membrane based on visual appearance. DLC had equipment in house to determine if a reduction in backpressure has been achieved and efforts concentrated on reducing the backpressure of the surface membrane.

There were essentially three "knobs" to turn in an attempt to reduce the backpressure of the membrane. They were the type of yarn used in the construction, the ratio of alumina slurry-to-yarn (the matrix ratio), and the spacing of the yarns on the surface of the filter body. Experiments were carried out to turn all three of these knobs in a systematic manner. The results of those experiments are presented in Figure 4.

To vary the yarn type, we chose to hold yarn denier constant at the level in the baseline filter, and vary the yarn twist. The two variations chosen are a twisted yarn and an untwisted yarn. It was expected that the untwisted yarn would flatten on the filter surface yielding a smoother membrane. The matrix ratio is determined by the size of the orifice in a stripper die, which controls the amount of alumina slurry applied to the yarn. To retain proprietary information regarding our process, we'll describe the matrix ratio values as "low" and "high." Finally, we can control the spacing of the surface yarns by adjusting the speed at which the yarn is wrapped around the support. To control proprietary information, we will refer to these yarn spacing as " $A$ " and "B," where "B" has fewer wraps per inch and a larger space between yarns. In these terms, the baseline filter membrane would be described as having been made with twisted yarn, high matrix ratio, and yarn spacing " $\mathrm{A}$ ". 


\section{$\Delta P$ versus OD Membrane}

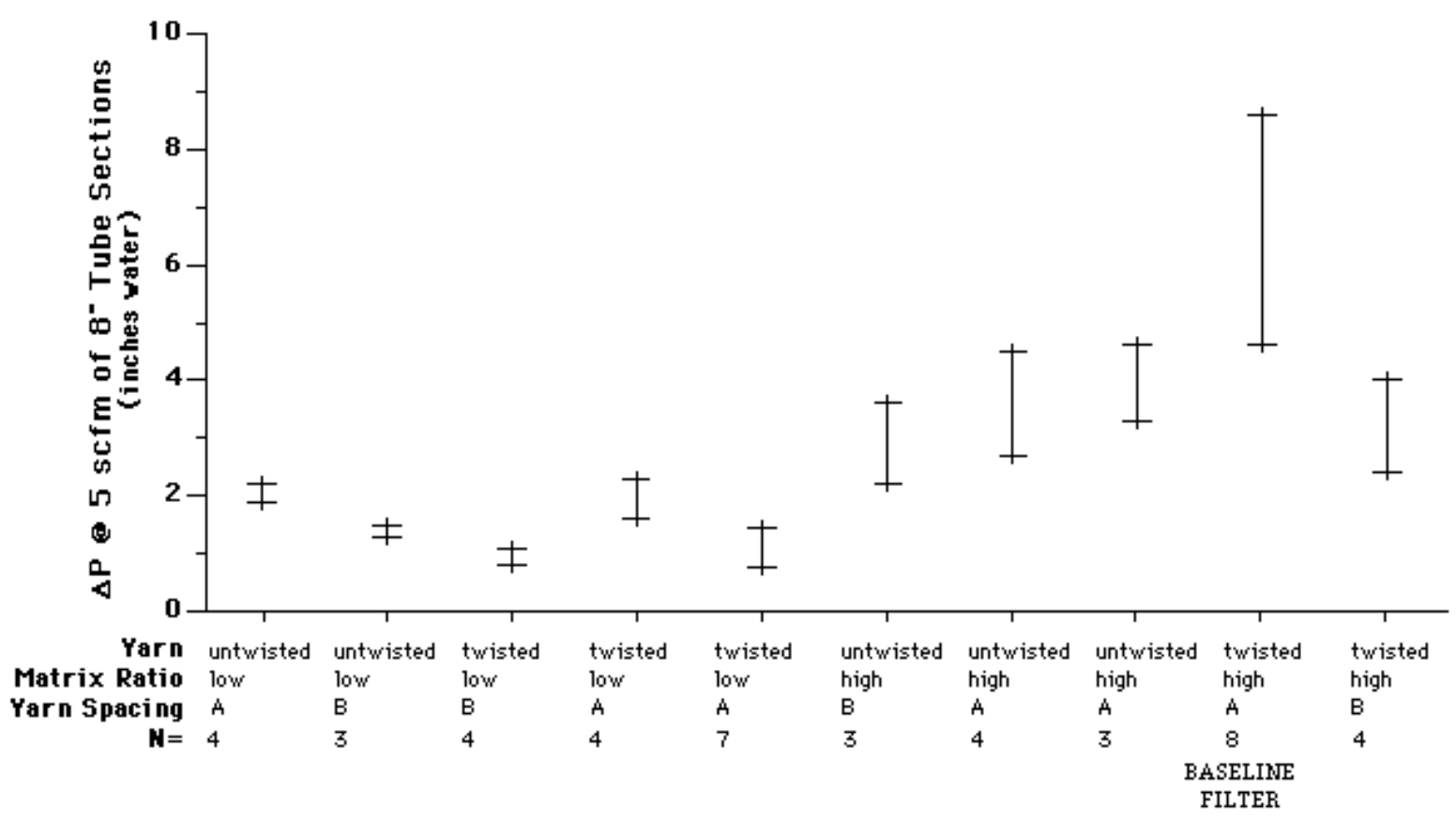

Figure 4 - Impact of three PRD-66 variables on backpressure $(\Delta P)$, where " $N$ " is the number of samples.

As seen in Figure 4, every combination of yarn twist, matrix ratio, and yarn spacing examined in these experiments resulted in a reduction in backpressure when compared to the baseline filter. In these experiments, the new combinations were also less variable than the baseline filter. It should be noted that the backpressure measurements were made are on 8" long samples taken from full size filters; the backpressure values presented in Figure 4 ARE NOT EQUAL TO values found on full filters, but they are proportional to them, so comparisons are meaningful.

There is no apparent correlation with yarn type seen in the data, a mild correlation with yarn spacing, and a strong correlation with matrix ratio. Lower matrix ratios have less alumina on the yarns, which probably results in less matrix bridging between adjacent yarns, and a more permeable membrane. It could not be determined if this "lower matrix ratio membrane" would provide an acceptable surface filtration. If it did, the results of these experiments indicate a reduction of surface membrane backpressure by a factor of four is possible.

The choice of yarn spacing is less clear. Yarn spacing 'B' generally gave slightly lower backpressure, but resulted in a visual appearance with randomly spaced gaps in the membrane. These gaps are likely to provide dust leak paths, and therefore poor filtration performance and may adversely effect cake release. DLC, therefore, elected to forego the small drop in backpressure and remain with the baseline yarn spacing. 
The untwisted yarn did flatten on the surface of the filter body as anticipated, but was more difficult to manufacture, leading to lower yields and higher costs. It also did not lead to an additional improvement in backpressure when used with the low matrix ratio.

Based on these results, DLC recommended the combination of a twisted yarn, low matrix ratio, and yarn spacing $\mathrm{A}$, because of the low backpressure, retention of a smooth membrane, and ease of manufacture of such filters. DLC manufactured two filters having these parameters for examination by Westinghouse Science and Technology Center; results are discussed in "3.2.5 Filtration and Permeability Testing".

Serious concerns were raised by Westinghouse over poor adhesion of the reduced backpressure membranes. In response, test were conducted with an intermediate matrix ratio, which was more adherent, but still had significantly lower backpressure then the baseline filter; data is shown below in Table 1. New samples were produced for testing; two-inch segments were cut from three locations (flange end, middle, and closed end). Each was sealed around the cut edge and shipped to W-STC for bench-scale permeability and particle filtration efficiency testing.

\begin{tabular}{|c|c|c|}
\hline Position within Candle & $\begin{array}{c}\Delta \mathbf{P} \text { of 8" Segment } \\
\text { (iwg, inches-water gauge) }\end{array}$ & $\begin{array}{c}\Delta \mathbf{P} \text { of 8” Baseline Segment } \\
\text { (iwg, inches-water gauge) }\end{array}$ \\
\hline Flange End & 2.4 & 4.7 \\
\hline Mid-Candle & 2.0 & 5.8 \\
\hline Tip End & 2.2 & 5.3 \\
\hline
\end{tabular}

Table 1 - Impact of "intermediate" matrix ratio on backpressure $(\Delta P)$ at $5 \mathrm{scfm}$

\subsubsection{Development of a Dual Membrane Candle Filter}

During earlier development efforts, Westinghouse expressed a desire to have a membrane along the inside, as well as outside, surfaces of the filter element; this configuration was referred to as a "dual membrane" filter. As a starting point for experiments leading to a "low backpressure, dual membrane" hot gas candle filter, we wound a bulk filter body identical to the baseline filter body, but with no inner or outer membrane. As shown below in Figure 5, this filter segment has an extremely low backpressure. This demonstrates that overall filter backpressure is dominated by the pressure drop at the surface membranes. Efforts were focused, therefore, on developing a "low backpressure, dual membrane" filter with "low pressure drop" outside diameter membranes. 


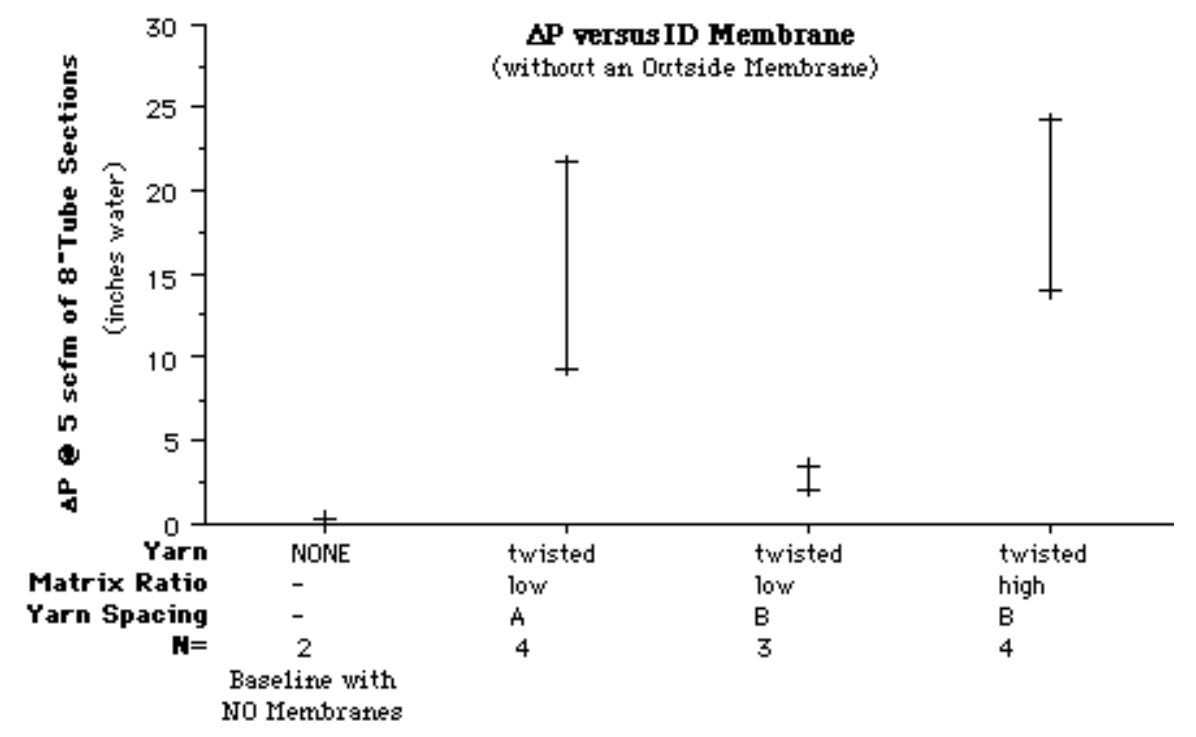

Figure 5 - Impact of inside diameter membrane on backpressure $(\Delta P)$, where " $N$ " is the number of samples.

In a manner similar to the experiments described above, experiments were completed to fashion an internal membrane by the same filament winding techniques used for the outer membrane. Instead of winding on the outer body of the candle filter, the internal membrane is wound on the mandrel, and the body of the candle is wound on top of the membrane. Since the wet yarns conform to the surface of the smooth mandrel, a very smooth membrane surface is obtained. We therefore expect excellent cake release from this inner membrane.

Figure 5 shows a backpressure dependence of inner membranes on both "matrix ratio" and "yarn spacing". No winding conditions could be found which would allow us to make a satisfactory inner membrane with an untwisted yarn. No gaps were formed in the membrane with yarn spacing "A" or "B". The combination of twisted yarns, low matrix ratio, and yarn spacing "B" for the inner membrane, was chosen based on the low backpressure. Samples of a dual membrane filter using these conditions for the inner membrane and the "medium matrix membrane" conditions described earlier are shown in Table 2.

\begin{tabular}{|c|c|c|}
\hline Position within Candle & $\begin{array}{c}\Delta \mathbf{P} \text { of 8" Segment } \\
\text { (iwg, inches-water gauge) }\end{array}$ & $\begin{array}{c}\Delta \mathbf{P} \text { of 8" Baseline Segment } \\
\text { (iwg, inches-water gauge) }\end{array}$ \\
\hline Flange End & 6.3 & 4.7 \\
\hline Mid-Candle & 9.9 & 5.8 \\
\hline Tip End & 10.3 & 5.3 \\
\hline
\end{tabular}

Table 2 - Impact of dual membranes on backpressure $(\Delta \mathrm{P})$ at $5 \mathrm{scfm}$. 


\subsubsection{Mechanical Testing}

In order to judge the effectiveness of our experiments to strengthen the flange region of our filters, a reliable mechanical property test was necessary. It was desirable for such a test to minimize the effect of machining damage incurred in fashioning the test specimen, and to be amenable for quality control in future production. Because PRD-66 hot gas filters are made by a process that produces only tubular shapes, it was impossible to manufacture a flat coupon that closely mimicked the internal structure of a PRD-66 filter. Only tests that use cylindrical samples, therefore, were considered. This limited the range to o-ring or c-ring tests. C-ring tests were subjectively evaluated, but cutting the 1-inch slot from the coupon incurred machining damage and an additional fixturing cost would have been necessary to achieve reproducible slot geometries. O-ring tests were ideal in that they required only two, easily controllable cuts to sample a tubular product. Since o-ring tension tests require more complicated and costly fixtures, a simple o-ring compression test was favored. Figure 6 shows a load deflection curve typical of o-ring compression tests carried out in this project.

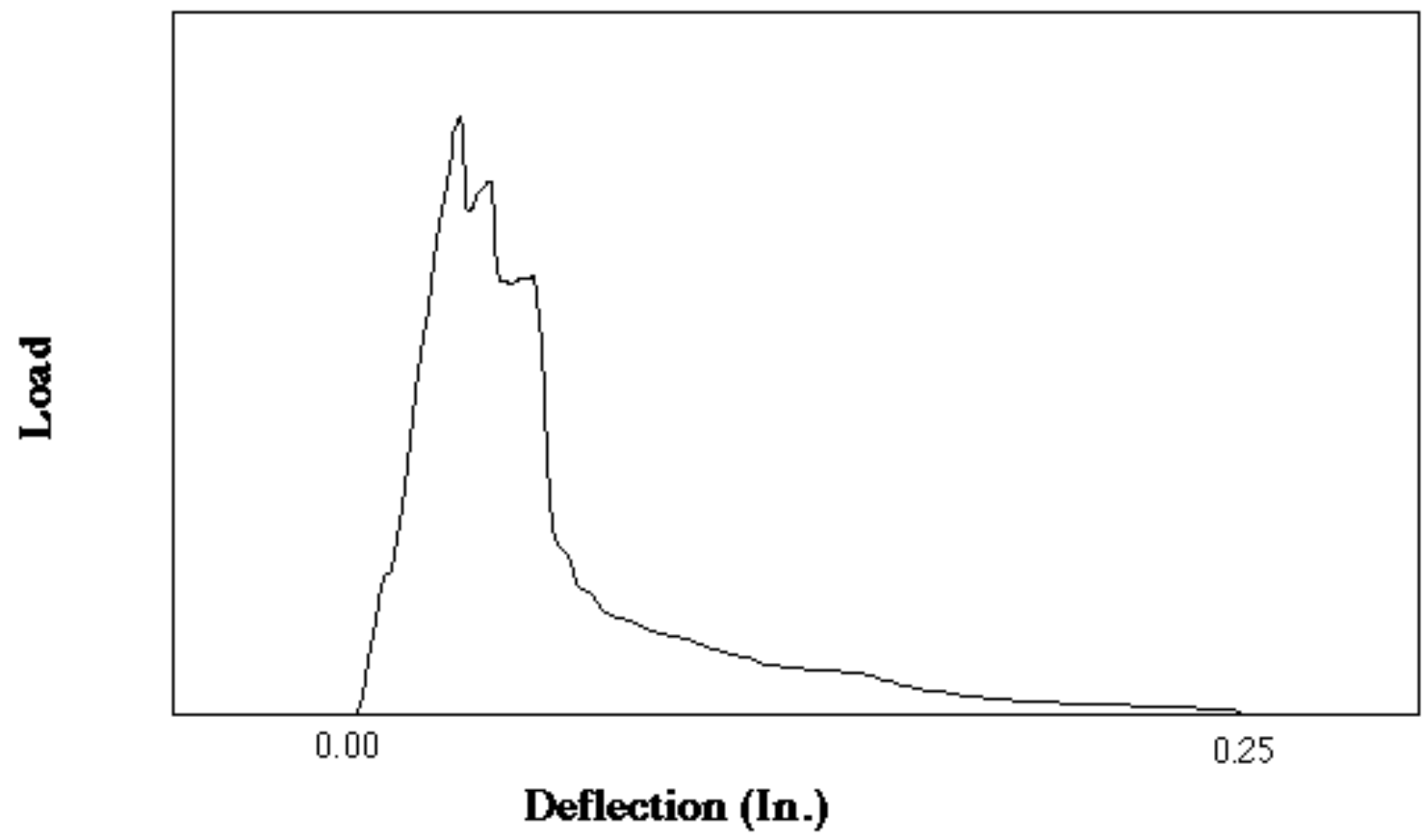

Figure 6 - Typical Load Displacement Curve for PRD-66 filter segment.

This displacement curve reveals a great deal of reloading and strain tolerance after peak load is achieved. Tests that were carried out until essentially no load resistance was encountered often had deflections as high as 0.25 inches, or roughly the same as the wall thickness of the sample. As shown in Figure 7, the samples were intact, though macroscopic cracks were readily visible. In the 100 or so mechanical tests conducted in developing this o-ring diametrical compression test, no sample fractured instantly into two or more pieces. 


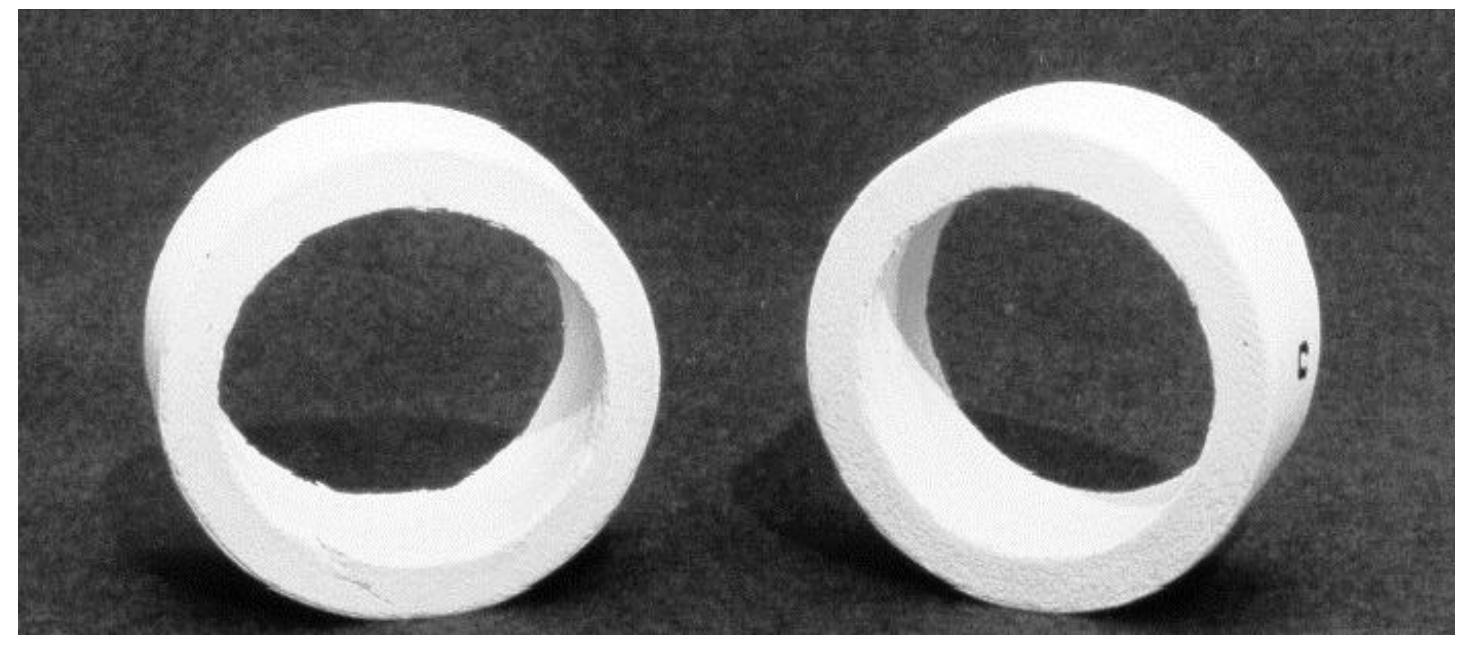

Figure 7 - O-rings AFTER (left) and BEFORE (right) diametrical compression testing.

The diametrical compressive strength was determined by the maximum peak at which the first crack occurred. To characterize PRD-66, forty-one 1-inch wide samples, from three different production filters were tested. The average crushing strength on the samples was 410 psi (std.dev. $=$ $38 \mathrm{psi})$. This is significantly lower than the results of Westinghouse Science and Technology Center's tests, which reported strengths of $1050 \mathrm{psi}$ on 3/4" wide o-rings. Unfortunately, the DLC records, which detailed the exact calculations used, were not available, however, a more accurate equation was adopted approximately a year after the original data was generated. In the later equation, developed by O.M. Jadaan, et al.10, stress is defined as follows:

$$
\begin{gathered}
\boldsymbol{\sigma}_{\theta=}=\frac{P}{2}\left[0.637 \frac{r_{a} y}{I}-\cos (\theta)\left(\frac{1}{\mathrm{~A}}+\frac{r a y}{I}\right)\right] \\
\text { where } \quad \begin{aligned}
I & =\frac{1}{12} b\left(r_{o}-r_{i}\right)^{3}=\frac{1}{12} b t^{3} \\
y & =r a-r \\
A & =b\left(r_{o}-r_{i}\right)=b t \\
P & =\text { load }
\end{aligned}
\end{gathered}
$$

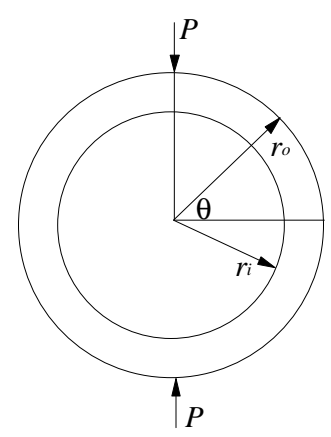

Where $I$ is the moment of inertia, $t$ is the thickness, and $A$ is the cross-sectional area. 
Using the new equation, a sample of the old data was recalculated as shown in Table 3. These strength values agree much better with data reported at W-STC. All data analysis that was conducted during Task 3, however, was performed with the old equations, which yielded lower values.

\begin{tabular}{|l|c|c|c|}
\hline & Load (lbs) & Old Strength Value (psi) & New Strength Value (psi) \\
\hline Filter \#316 (n=13) & 33 & 417 & 1057 \\
\hline Filter \#317 (n=16) & 35 & 369 & 934 \\
\hline Filter \#318 (n=16) & 41 & 423 & 1071 \\
\hline
\end{tabular}

Table 3 - Average o-ring diametrical compressive strength (" $n$ " is the number of samples).

A Weibull analysis, shown in Figure 8, was conducted on the original data after calculating the failure strengths of each of the o-rings at the point of maximum stress on the load/deflection curve (Figure 6). The resulting failure stresses were then used to obtain parameter estimates associated with the underlying population distribution.11 PRD-66 behaved as expected for a porous ceramic material, with a Weibull modulus around "4". Significantly more data would be necessary to correct for statistical bias errors and calculate confidence bounds.

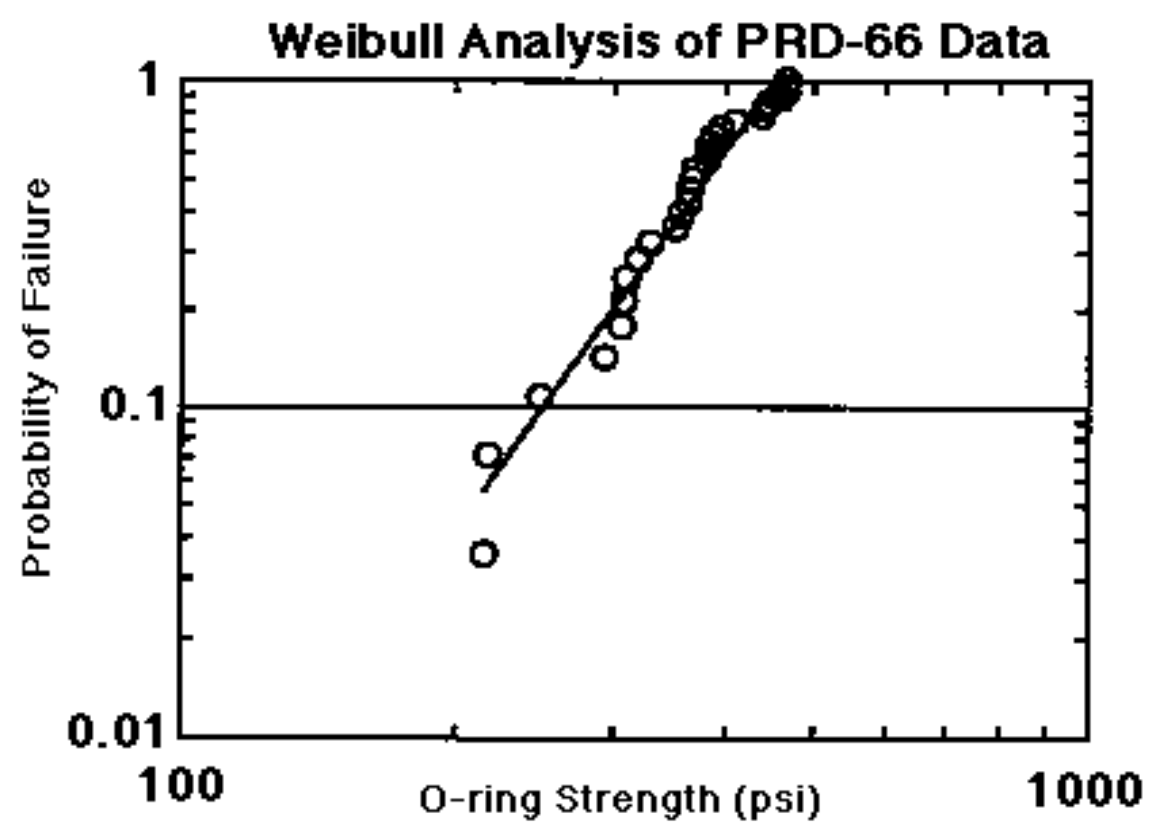

Figure 8 - Weibull Analysis of PRD-66 candle filter segment.

Additional o-rings were tested at various rates of applied stress, as determined by the crosshead speed of the apparatus. When the average strengths were plotted in Figure 9, their was 
no obvious strain rate dependence for PRD-66, additional data would be required, however, to verify statistical significance.

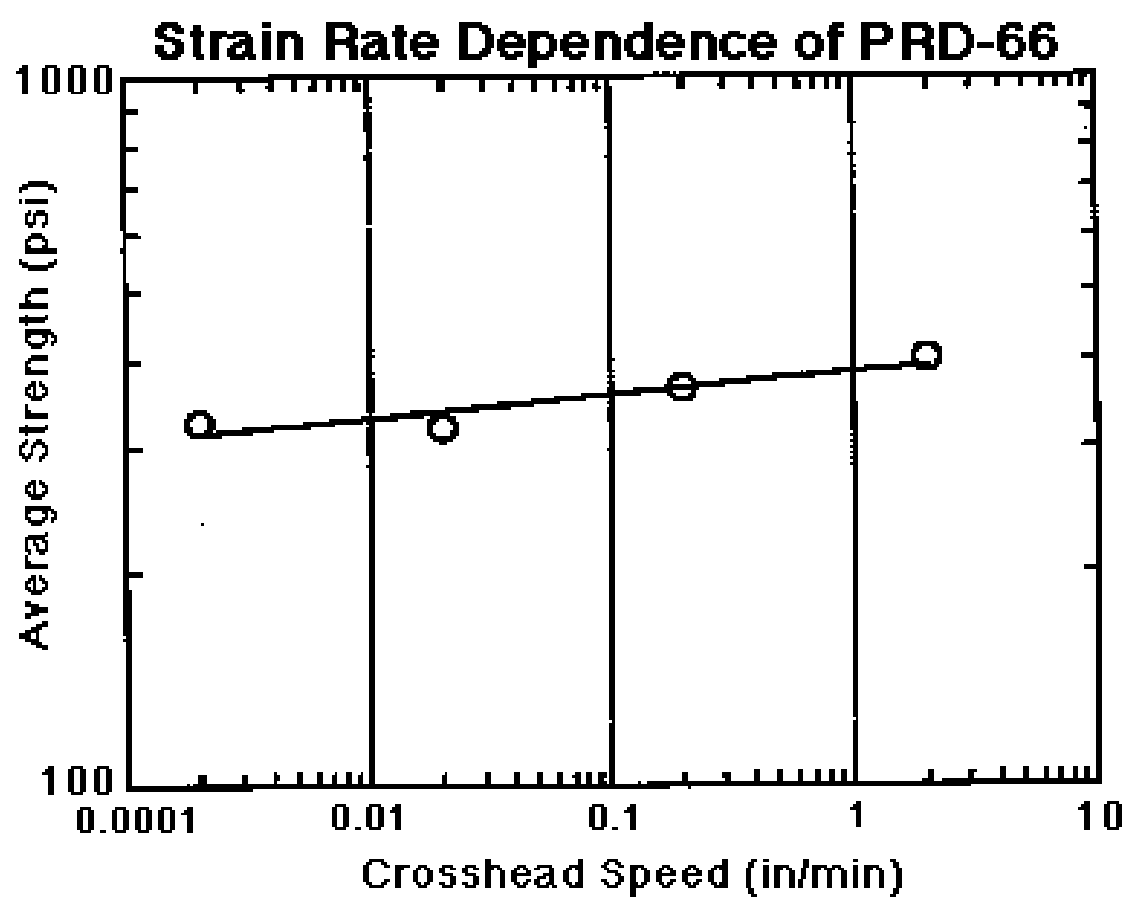

Figure 9 - Strain rate dependence of 0 -ring crushing test.

\subsubsection{Strengthened Flanges}

In previous experiments, it had been shown (although somewhat qualitatively) that selective reinforcement of PRD-66 filters can be obtained by adding slurry to portions of the filter in need of reinforcement after winding the filter body. This was of particular importance in view of early tests conducted at Westinghouse (and reported verbally to DLC) in which failure of the filter element occurred just below the flange. Since that time, the holder assembly was redesigned by W-STC and a method was developed by DLC to add controlled amounts of slurry to the areas requiring reinforcement.

Filter samples were fabricated with a range of slurry additions $(10,15$, or $20 \mathrm{cc})$ introduced to portions of the bulk filter body. Three different slurry viscosities were also tested to examine whether the infiltrated slurry stayed where it had been applied or migrated into adjacent regions. To control for filter-to-filter variations, replicate samples were taken from several different filter bodies, and at different points along the body. 
As seen in Figure 10 and Figure 11, there is a strong positive correlation between both the "weight gain" and "volume of added slurry" with o-ring crushing strength of 1-inch segments of the filter. Overall, strength increased from about 400 psi for uninfiltrated sections to about 600 psi for fully infiltrated samples, about a $50 \%$ increase in strength.

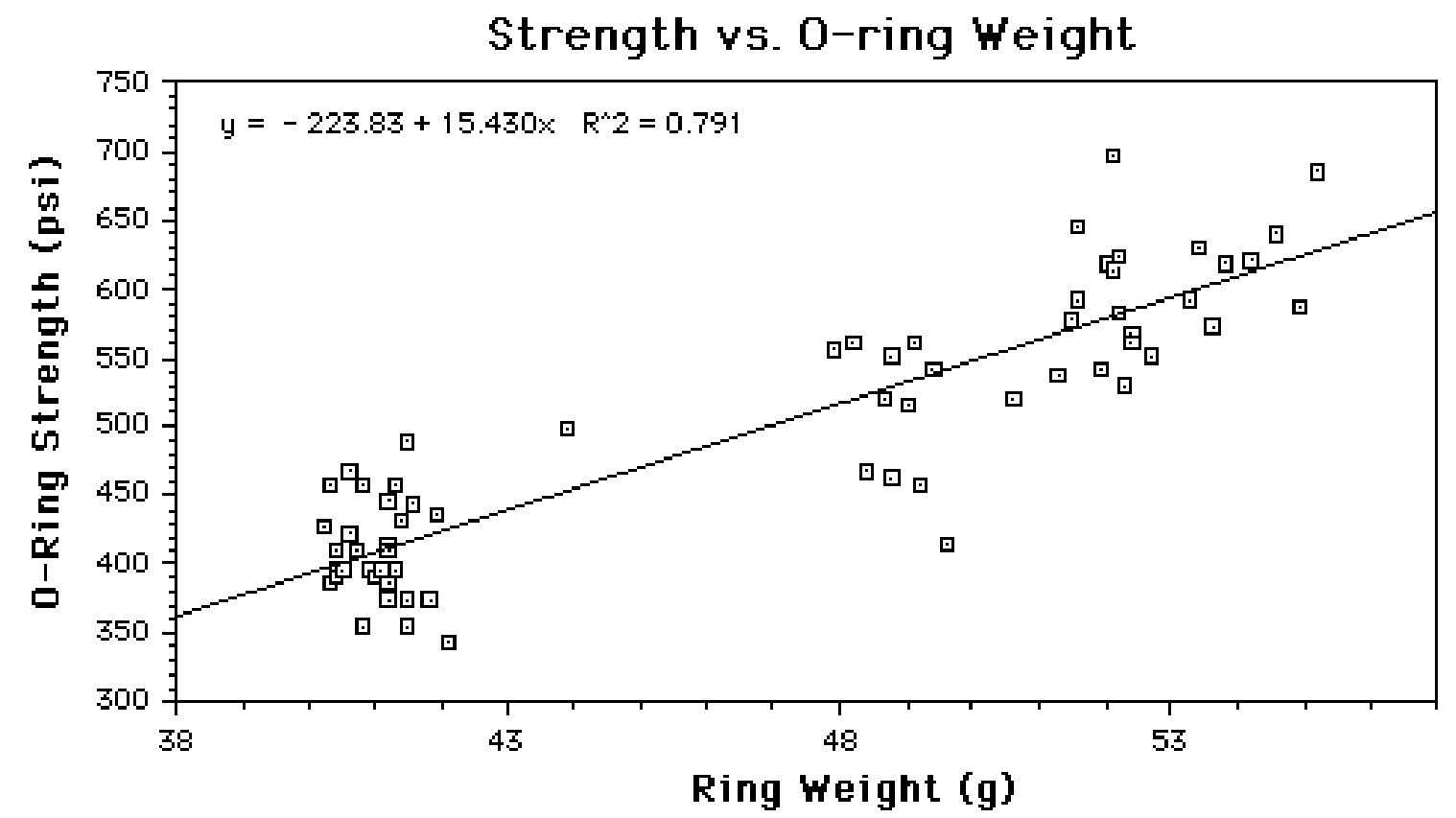

Figure 10 - O-ring diametrical compressive strength versus ring weight.

Higher viscosity slurries (Figure 11) achieved higher strengths with less slurry addition, and lower viscosities took more slurry to attain the same strength. This is probably due to migration of the slurry out of the test segment into the regions adjacent to it, which would result in less effective reinforcement. 


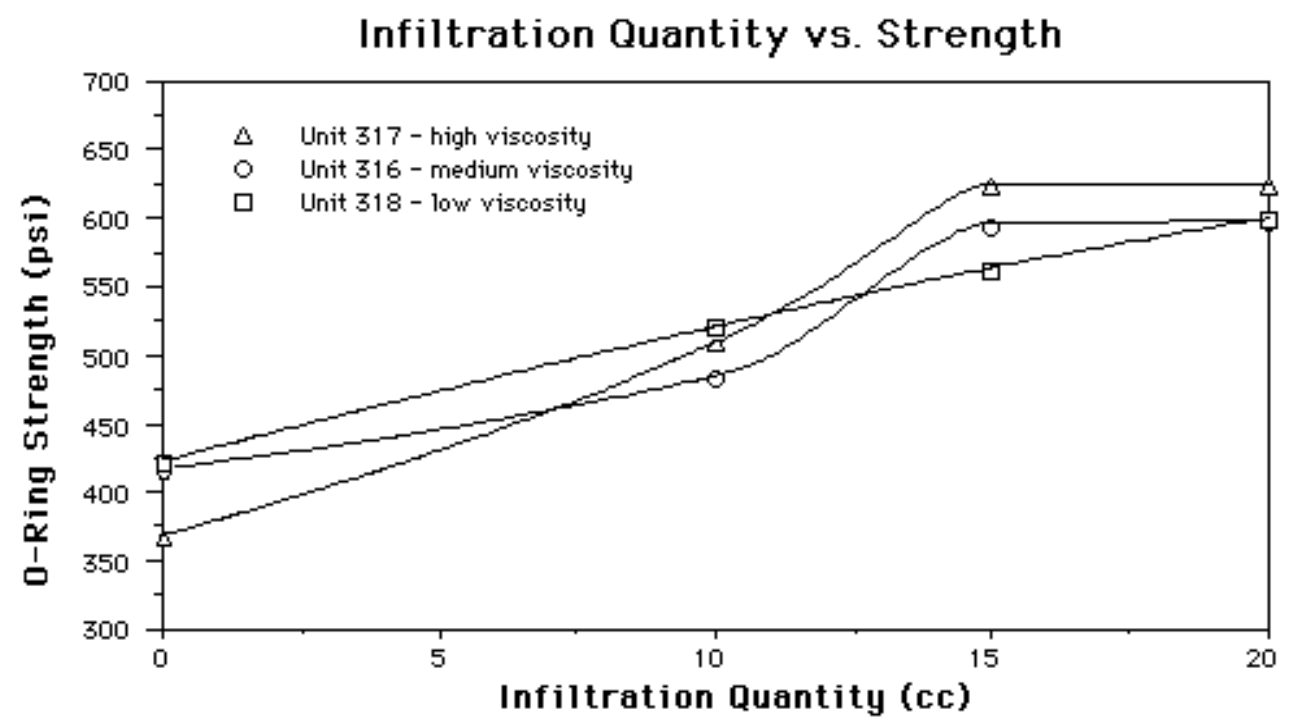

Figure 11 - O-ring diametrical compressive strength vs. amount $\&$ viscosity of infiltrate

There appears to be a greater tendency toward brittle failure with the infiltrated material, but, as shown in the load displacement curve of Figure 12, there is still quite graceful failure. We interpret the more triangular shape plot after maximum load (compared to Figure 6) as an indication of more brittle failure, but the fact that there is significant reloading after "peak load" still suggests graceful failure. As with the uninfiltrated material, these samples never broke into two pieces, even with deflections as large as $1 / 4$ inch and as many as four independent cracks per specimen.

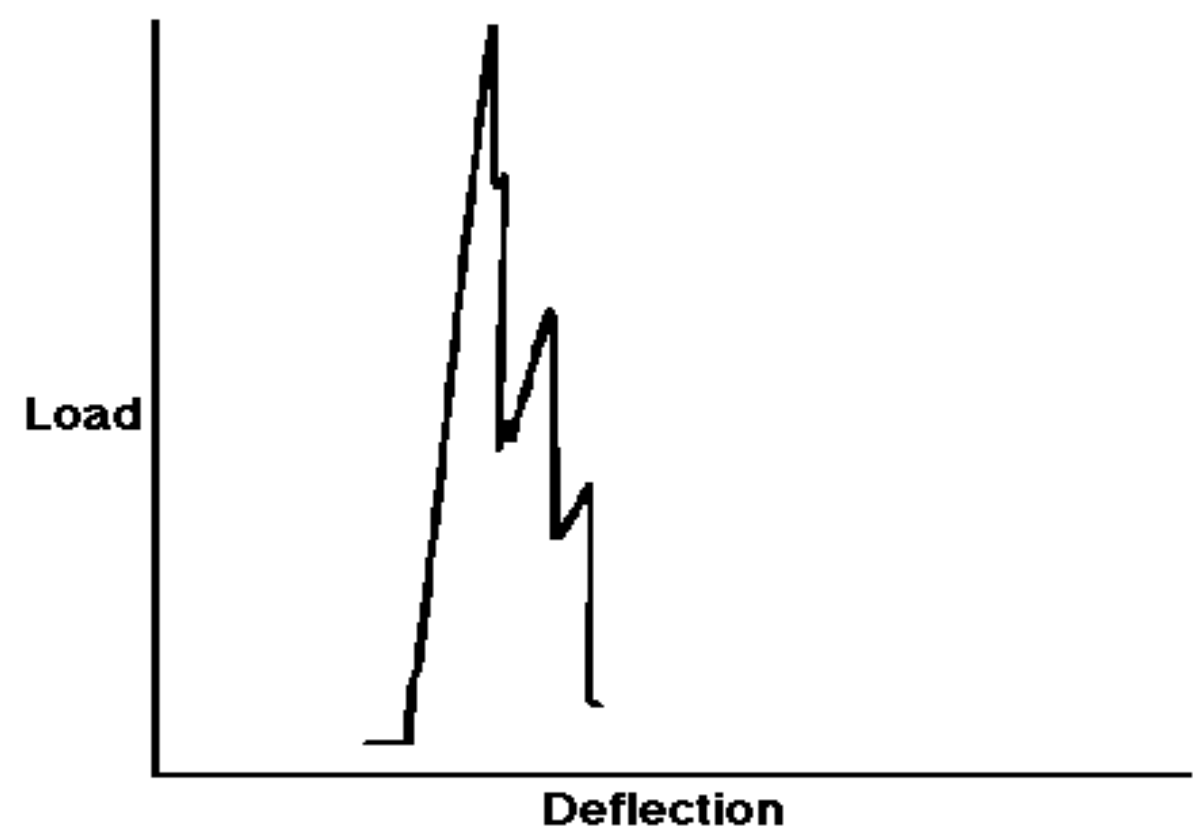

Figure 12 - Load Displacement Curve for infiltrated PRD-66 filter segment. 
Seeing no real negative factors in using this new infiltration technique, and a significant benefit, strengthened flanges were incorporated into all three of the improved filter designs, mentioned earlier. Two "baseline" candles with the improved reinforcement technique at the flange were submitted to Westinghouse for testing.

\subsubsection{Filtration and Permeability Testing}

The following information, with regard to testing performed by Westinghouse Science and Technology Center (W-STC), was conducted under a subcontract between DLC and Westinghouse12. A full copy of the Final Report is provided in Appendix 3.

Preliminary tests were conducted by Westinghouse Science and Technology Center on 2inch long filter segments that had low pressure and dual membranes. Dust was delivered to each sample's outside diameter at room temperature for $\sim 3$ minutes. Both the clean ID appearance, as well as the absence of detectable fines in the off-gas stream indicated excellent particle collection efficiency, by Westinghouse standards. When a tested specimen was fast-fractured, fines were evident below the outside diameter surface. Penetration within the 7-mm thick wall was apparent to a depth of $1-3 \mathrm{~mm}$.

As mentioned earlier, DLC fabricated the following 1.5-meter candles for testing: two with improved (low pressure) outside membrane only, two dual membrane candles, and two "baseline" candles, ALL with strengthened flanges. Westinghouse performed room temperature gas flow resistance measurements on all six candles; results are show in Figure 13. These results parallel measurements conducted at DLC. 


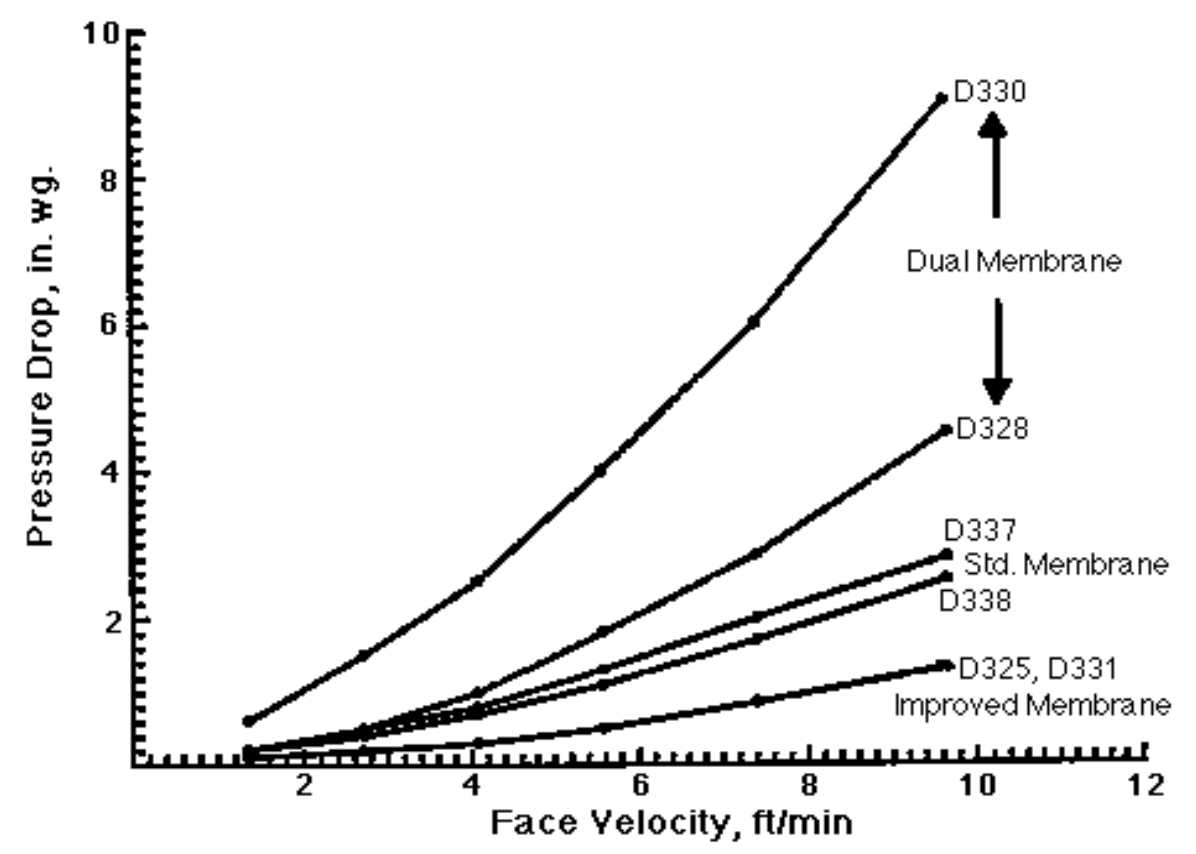

Figure 13 - ㅁ-STC Room Temperature Gas Flow Resistance measurements of 1.5-meter PRD-66 filter elements with various membranes. ${ }^{12}$

Westinghouse concluded that the "baseline" and the reduced backpressure membrane filters had flow restrictions within their specification range. The flow restriction of the two dual membrane filters did not agree with each other and one exceeded the pressure drop specification of $<1$ in-wg/fpm.

After two hours of high temperature exposure in Westinghouse's HTHP facility, outer membranes on the reduced backpressure and dual membrane filters delaminated. This was the most probable failure mode of these candles. The "strengthened flange" filter, which had the baseline surface membrane, did not delaminate. 


\subsection{Field Testing of "Baseline" PRD-66 Filter Elements}

Prior to the beginning of this program, PRD-66 hot gas candle filters (baseline filters) were tested at Westinghouse Electric Corporation's Science and Technology Center. ${ }^{2}$ Testing on two-inch filter segments confirmed that PRD-66 filters had acceptable particle filtration efficiency and permeability characteristics in lab scale testing. Westinghouse then exposed full-size, 1.5meter candle filters to simulated coal combustion filtration conditions in their high temperature high pressure (HTHP) test chamber. That testing confirmed that full-scale candle filters also performed well in filtration efficiency and permeability. Accelerated pulsing and process interruption testing revealed the need for strengthening of the flange region of the filter. After DLC took steps to increase the strength of the filter's flange, further accelerated tests which simulated 6000 hours of filtration were successful.

To identify the thermal/chemical stability of the PRD-66 material, W-STC subjected 10" mini-candles to 400 hours at $870^{\circ} \mathrm{C}$, in a 5-7\% steam/air environment at $1 \mathrm{Atm}$. Additional samples were subjected to 400 hours at $870^{\circ} \mathrm{C}$, in a $20 \mathrm{ppm} \mathrm{NaCl} / 5-7 \%$ steam/air environment at 1 Atm. X-ray diffraction was used to compare the crystalline compositions of the materials. Neither of the test conditions had any measurable effect on the PRD-66 material. ${ }^{3}$

\subsubsection{Tidd Test Segment 4}

After the testing at Westinghouse, three PRD-66 candle filters were placed into field testing at American Electric Power's Tidd Pressurized fluidized bed combustor (PFBC) filter vessel. The PRD-66 candles were placed in the middle array of the vessel. They were exposed to temperatures up to $760^{\circ} \mathrm{C}$ and operated for the entire duration of the test segment, 1700 hours with ash loading of 3200 ppmw. All three of the PRD-66 filters survived the test segment and suffered no damage. Upon inspection of the filters after exposure, only a loose, thin (approximately 1/8" thick) ashcake clung to the candles. Despite significant ash bridging problems in the test, no ash bridges were found on the PRD-66 candles. Mechanical property tests performed by Westinghouse on ring segments cut from the exposed filters showed no decrease in mechanical properties after the 1700-hour exposure. The only significant negative finding in the test was that the wall of the PRD-66 filters had become filled with trapped ash. At the time, this was attributed to ash penetration from the inside of the filter, due to ash reaching the "clean side" of the filter vessel from other broken candle filters tested in the same plenum. ${ }^{2,8}$ 
From the results of this test segment, DLC concluded that PRD-66 candle filters were resistant to attack by the corrosive atmosphere resulting from coal combustion. Further, it was concluded that PRD-66 filters had the necessary mechanical strength to survive filtration and backpulse cleaning for at least 1700 hours of operation. The complete retention of mechanical properties in post-exposure testing suggests that under the conditions in Tidd Test Segment 4, significantly longer useful lives would be possible.

\subsubsection{Tidd Test Segment 5}

Concurrent with the development of the low-pressure and dual-membrane filter elements under this program, twenty-two "baseline" PRD-66 candle filters (identical to those used in Test Segment 4) were placed in service in Tidd Test Segment 5. After the test, it was discovered that all of the PRD-66 candle filters had experienced significant damage. Two types of failure were observed. The first was a classic flange failure, with filters broken in the holder area where the flange transitions to the filter body. The second failure mode was observed mid-body, with approximately half the filter body remaining intact. In this failure mode, "divots" were taken out of the filter body, appearing as lenticular avulsions greater than a millimeter deep, as shown in Figure 14. In filters with mid-body failures, fracture occurred at these thinned spots in the body wall, often where a "divot-in-a-divot" had removed most of the wall thickness. ${ }^{2,8}$

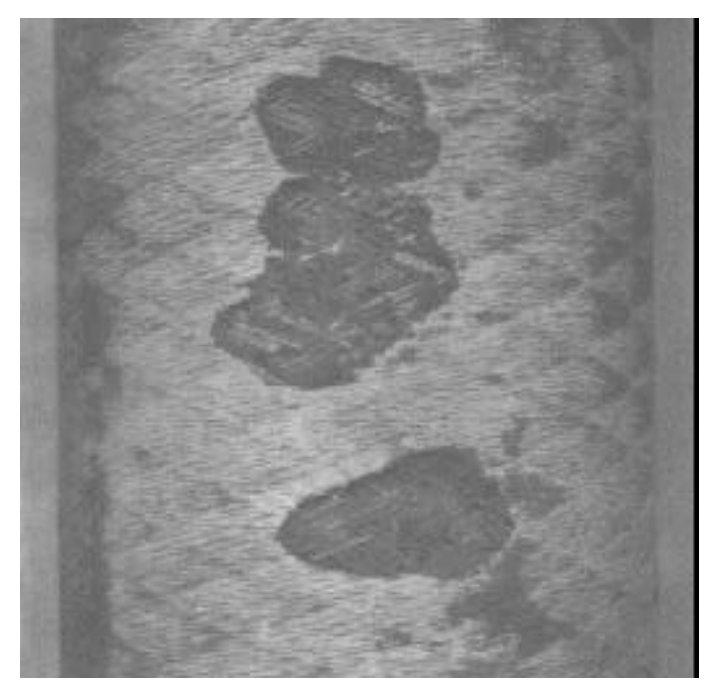

Figure 14 - "Divots" in PRD-66 filter tested in Tidd Test Segment 5.

\subsubsection{Analysis of Field Exposed Elements (Subtask 3.4)}

To understand the cause of the discrepancy between the results of Tidd Test Segments 4 and 5, DLC undertook Task 3.4 of this program, entitled "Analysis of Field Exposed Filters". This 
task was carried out in five phases: Consultation, Elimination of Known Faults, Hypothesis Formulation, Hypothesis Verification, and Correction. ${ }^{8}$

\subsubsection{Phase 1 - Consultation}

In the Consultation phase, DLC held discussions with numerous experts in the field of hot gas filtration, including Ted McMahon, Rich Dennis and Dwayne Smith of FETC, Mary Anne Alvin and Rich Newby of Westinghouse Science and Technology Center, Tina Watne and John Holmes of the University of North Dakota's Energy and Environmental Research Center, and Dick Tressler of Penn State. Valuable evidence and insight was gained from these discussions, which is incorporated into following summary.

\subsubsection{Phase 2 - Elimination of Known Faults}

In Phase 2, DLC undertook detailed evaluations of all the manufacturing records for filters supplied to Tidd Test Segment 5 to seek any anomalies in manufacturing which might explain the differences in performance. While some minor changes in the process were found, no process variations correlated with performance. X-ray diffraction tests on the filters fired in the same run with Test Segment 5 filters showed no difference with those in Test Segment 4.

\subsubsection{Phase 3 - Hypothesis Formulation}

Unable to find any significant differences in the filters, Phase 3 focused on physical evidence found in filters which survived Test Segment 5 in whole or in part, and documented differences in run conditions between Test Segments 4 and 5. As shown in Table 4, there were significant differences between Test Segments 4 and 5.

\begin{tabular}{|l|l|l|}
\hline Test Segment & Tidd 4 & Tidd 5 \\
\hline Test Duration & $1700 \mathrm{hrs}$. & $1100 \mathrm{hrs.}$ \\
\hline Survival Rate & $100 \%$ & $10 \%$ \\
\hline Ash Cake & Thin, uniform & Thin, patchy \\
\hline Damage & None & $\begin{array}{l}\text { Divots, mid-body } \\
\text { Broken, flange }\end{array}$ \\
\hline Bridging & None & None \\
\hline Operating Temperature & $660-760^{\circ} \mathrm{C}$ & $760-845^{\circ} \mathrm{C}$ \\
\hline Ash Loading & $3,200 \mathrm{ppmw}$ & $18,000 \mathrm{ppmw}$ \\
\hline Primary Cyclone & De-tuned & Inactive \\
\hline
\end{tabular}

Table 4 - Comparison of test conditions in Tidd Test Segments 4 and 5. 
Ash loading increased from 3,200 ppmw to 18,000 ppmw because of the inactivation of the primary cyclone upstream of the filter vessel. The mean particle size of the ash increased significantly. The highest run temperature increased from 760 to $845^{\circ} \mathrm{C}$. Different adsorbents and coals were used. In Test Segment 4, the PRD-66 candle filters were placed in the middle array, while in Test Segment 5, they were in the top array. Two failure modes were observed. One was a classic flange failure, with the fracture locus high up in the holder. These filters, in order to remain identical to the ones tested in Test Segment 4, did not use the selective reinforcement of the flange area described in Section 3.2.4. This reinforcement technique would have increased the strength of the PRD-66 material by about 50\%. A second, more puzzling failure, was that found in along the body of the filters. The physical evidence seen on the filters included "divots", as shown in Figure 14.

"Divots" are pieces of the candle filter membrane and body, avulsed from the filter. Such "divots" were found aligned along the filter body on roughly opposite sides. A "divot" was also found under the sock and holder, which eliminates mechanical impact as a cause of the damage. There was no visible evidence of corrosion. The filter body walls were filled with ash, as they had been in Test Segment 4. The body of the filter was covered with a thin layer of loose ash, roughly $2 \mathrm{~mm}$ thick in most regions. There were also denser ash deposits, aligned with the "divots" described above. All "divots" were packed with dense ash, though some ash-packed "divots" were covered with loose ash. Finally, in Test Segment 5, all filters of all types in the top array were somehow "glued" in place (strongly adhered to their holders). This was not observed in the middle or bottom arrays. Filter segments tested by Westinghouse showed no decrease in mechanical properties after exposure. Finally, micrographs taken at EERC by Tina Watne showed inclusions of a white material, identified by EDX as containing magnesium, calcium, sulfur, and oxygen, well inside the filter body, see Figure 15. This white deposit was of a physical size far too large to have penetrated the undamaged filter above it intact. Undamaged filter areas showed no such deposits.

Based on this evidence, a hypothesis of the failure mechanism of PRD-66 candle filters in Test Segment 5 was formulated. Despite earlier results of room temperature and high temperature tests to the contrary, ash that contained adsorbent penetrated the surface membrane of the PRD-66 filters. This ash then became trapped in the bulk filtering body of the candle. Once trapped there, it was subjected to long term exposure of hot $\mathrm{SO} 2$ gas, causing in situ sulfation of the ash to calcium and/or magnesium sulfates in the pores and microcracks of the filters. 


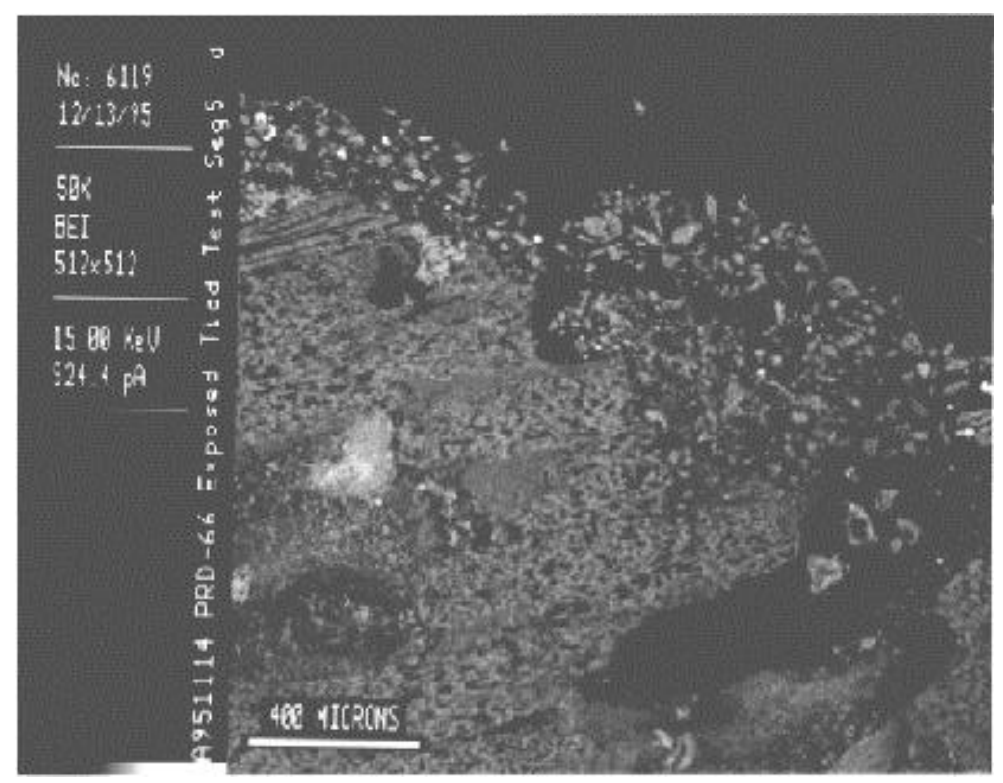

Figure 15 - White deposit (middle left) in vicinity of "divot" (upper right).

Once lodged in a microcrack at high temperature, these deposits could change in size by several mechanisms. One possible damage mechanism is by thermal expansion and contraction of the sulfate deposit during process interruptions, of which there were several in Test Segment 5. A second possible mechanism is by crystal growth from the hydration of sulfates during cooling in a moisture-containing atmosphere, which also would occur on process interruptions. Figure 16 shows how the unit-cell volume of anhydrous magnesium sulfate increases as it picks up waters of hydration.

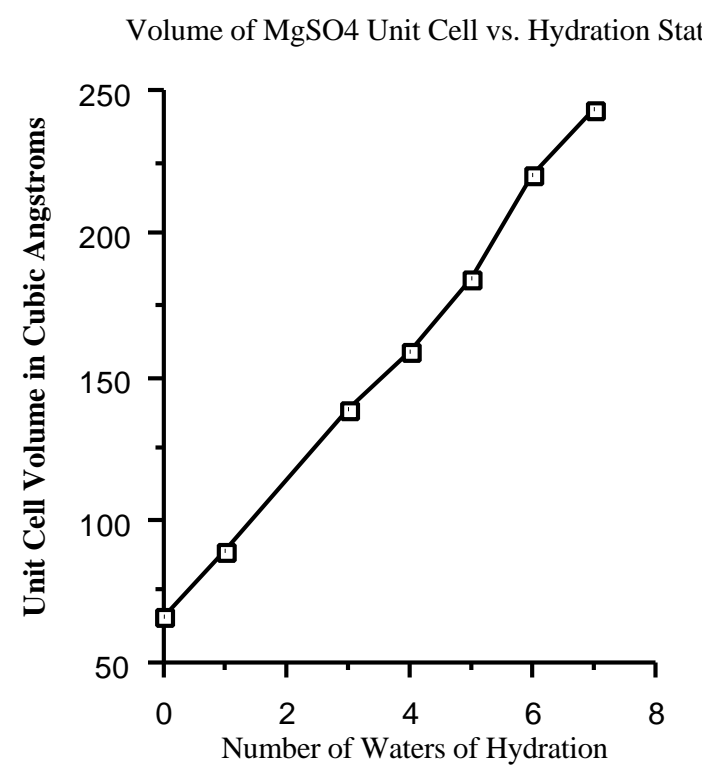

Figure 16 - Unit-cell size of magnesium sulfate versus state of hydration. 
The roughly four-fold volume increase associated with formation of the hexahydrate salt would induce a linear strain in a microcrack of over $150 \%$, far larger than the strain tolerance of most ceramics. By either of these mechanisms, severe internal stresses could be placed on the filter body, causing localized failure near a sulfate deposit. In areas where multiple deposits formed, a "divot-in-a-divot" could occur, either fracturing the wall or weakening the wall enough to cause mechanical failure during a backpulse.

\subsubsection{Phase 4 - Hypothesis Verification}

In Phase 4, DLC set out to verify that 1) this hypothesis is in keeping with the known conditions of Test Segment 5, and 2) the possibility of penetration of ash through the surface membrane, contrary to previous test results. DLC found that all conditions necessary for the hypothesis to be true existed in the Tidd test conditions. All that was required was the presence of trapped ash in the filter, the presence of gas phase $\mathrm{SO} 2$, and moisture, plus rapid temperature excursions. All these circumstances can be verified from knowledge of the system, the run history, and physical examination of the field exposed filters. To verify that it was possible that ash leaked through what was thought to be 'leak proof' surface membrane, DLC devised a roomtemperature test of surface filtration characteristics more rigorous than the ones it had previously passed. In the previous tests, filter segments were exposed to gas flows containing ash. Once a smooth filter cake built up, it was supposed that the ashcake would strongly adhere and then take over filtration. A sample passes the test if no ash penetrates to the inner diameter. Since physical evidence from Tidd Test Segments 4 and 5 showed that the ashcake was thin and only loosely adhered, DLC worked under the assumption that, the surface of the PRD-66 filters released the ash essentially completely on each backpulse. To mimic this ash removal in DLC's laboratory, after exposing filter segments to ash by applying a vacuum to the inner diameter, the resulting ashcake was physically removed with light brushing. This ash exposure/cleaning cycle was repeated 25 times. The intent was to simulate the effect of complete ashcake release after a series of cleaning backpulses. Figure 17 illustrates the apparatus used to conduct this test.

In this test, ash consistently penetrated the membrane of the "baseline" filter and accumulated in the filter wall. Figure 18 shows an example of a 2"-segment, exposed to 25 PIT cycles, viewed with transmitted light; the light source had been inserted into the sample and the examination was performed in a darkened room. 


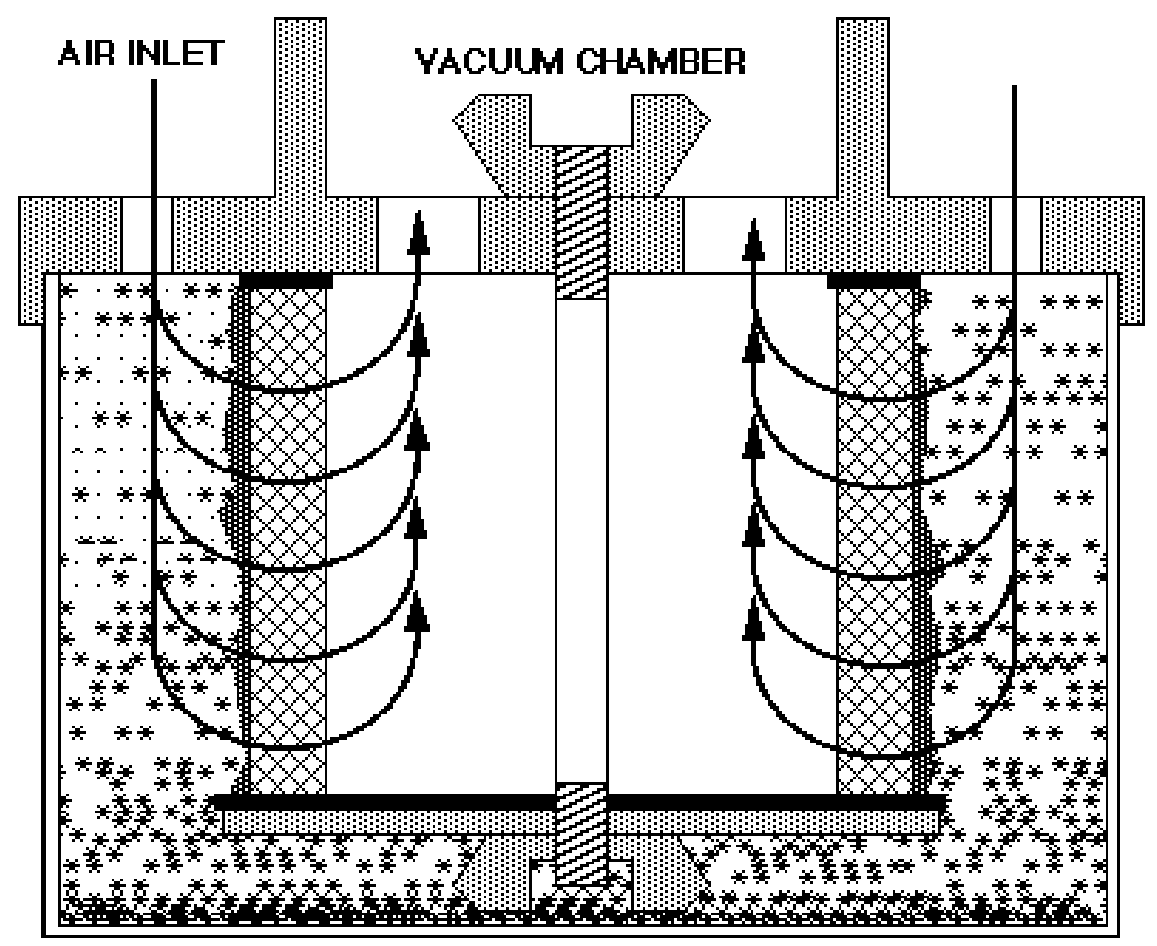

Figure 17 - Particle Infiltration Test (PIT) Device

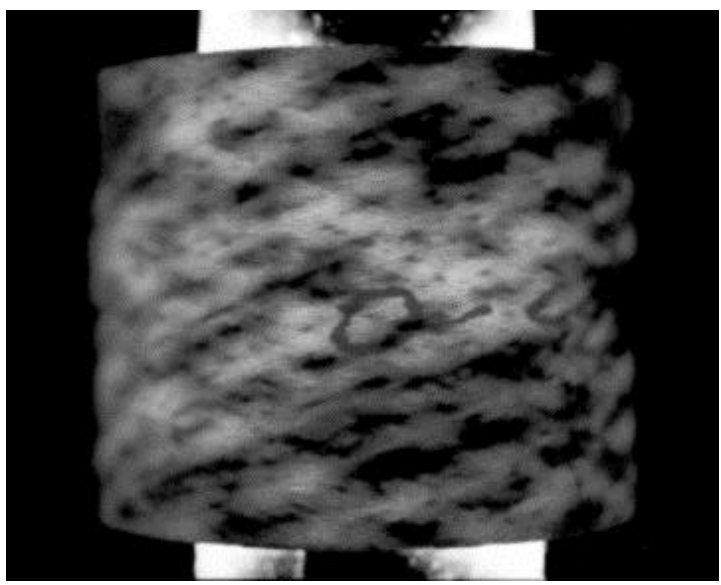

Figure 18 - PIT-exposed sample viewed in transmitted light.

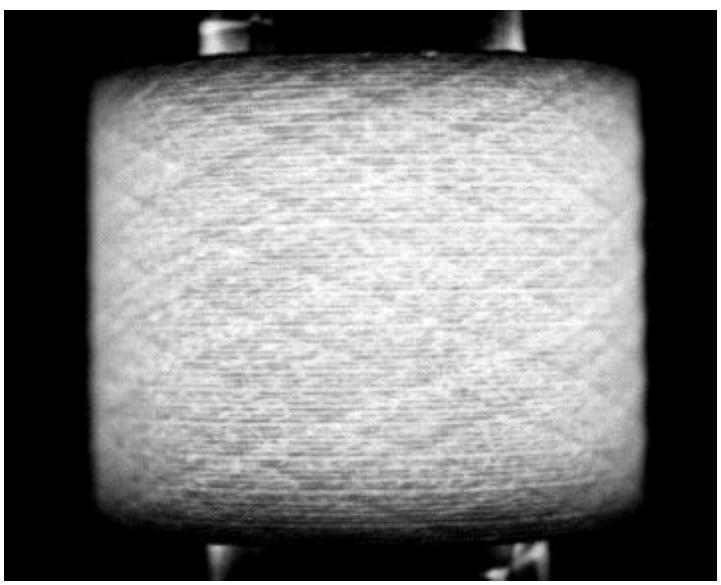

Figure 19 - Untested sample viewed in transmitted light.

When compared to an untested filter segment (Figure 19), areas of ash infiltration appear as dark streaks and spots; in the case of the "baseline" membrane, these areas are many and widespread. Even after the extensive penetration shown in the figure, however, ash still did not penetrate to the inner diameter after 25 cycles. This indicates that the bulk filtering body does trap ash in the wall. Because of the expense associated with recreating the in-situ sulfation of the penetrated ash, no such experiments were conducted. 
Further verification of this hypothesis was found by Westinghouse's independent investigation of the failure mechanism. Westinghouse discovered differences in the ash adhered to the filters and uncleaned surfaces in the top array, versus the ash in the two lower arrays. They verified that the filters of the top array were 'glued' in place. Westinghouse also reported the presence of magnesium sulfate hexahydrate in the ash, as found by X-ray diffraction, on uncleaned, stagnant surfaces of the top array, such as the holders and tubesheet. As described above, DLC hypothesized the formation of magnesium sulfate hexahydrate in the filter body as a potential cause of damage, without formally verifying the existence of the compound by XRD. Westinghouse's proof of the formation of the hexahydrate salt verifies that actual system conditions present in Test Segment 5 could cause its formation, and therefore supports the likelihood of DLC's hypothesis. The fact that no such compound was found in the middle array could explain why ash-filled PRD-66 candles in the middle array of Test Segment 4 showed no damage.

The presence of factors that may have contributed to the formation of "divots" was confirmed, but this theory alone could not explain the presence of "divots" in localized areas. The PIT evaluation indicated that ash penetration would occur in over half of the filter surface and examination of the exposed filters showed that the ash was thoroughly imbedded throughout the wall of the entire unit. As a percentage of the outside diameter, the "divots" would account for less than $5 \%$ of the surface. A significant contributing factor may have been the presence of regions, within the wall, of poor interlaminar strength. When a PRD-66 candle filter is cut into rings, it is common to observe regions where adjacent layers of yarn have separated from each other, as shown in Figure 20. Occasionally, these defects might extend approximately a quarter of the way around the circumference, and continue for 1-2" inches along the length of the filter element. They have been observed at random depths and positions within the support body and could never be correlated with any process variables.

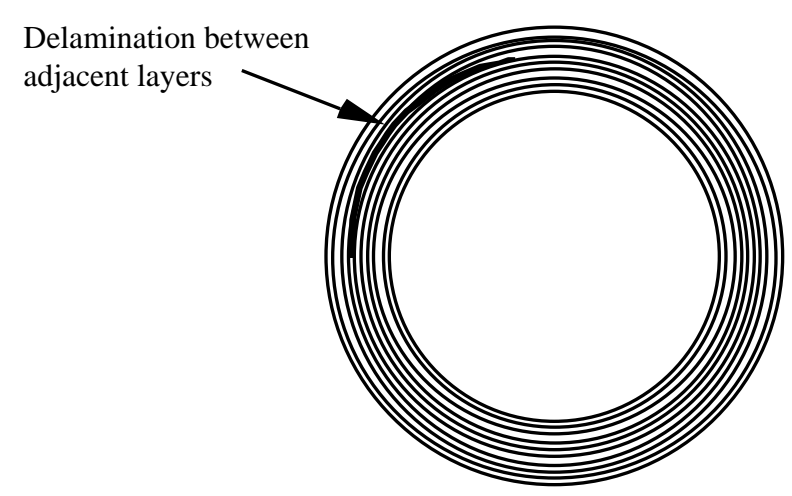

Figure 20 - Exaggerated illustration of a PRD-66 delamination 
It is possible that the "divots" were caused by the combined presence of three things: an environment conducive to sulfate formation and hydration, ash entrainment, and localized interlaminar weaknesses. Since DLC has no control over the PFBC environment, corrective action was focused on improving the surface filtration quality of the membrane and reducing the presence of delaminating within the support wall.

\subsubsection{Phase 5 - Correction}

The composition of the "baseline" slurry was fumed alumina, calcined A-17-grade alumina, and deionized water. Observations made during Subtask 3.1 suggested the resulting alumina matrix might not have had adequate bonding strength. It was also noted that in the green state (dried, but not fired), bonds between coated filaments could be damaged when removing the filter from the mandrel. An alternate composition was evaluated in which the fumed alumina in the slurry was replace by aluminum chlorohydrate $\left(\right.$ Chlorhydrol $\left.^{\circledR}\right)$, as an alumina precursor. This ingredient imparts significant "green strength", unfortunately environmental controls were necessary to deal with the evolution of $\mathrm{HCl}$ that results during heating. To remove this hazardous byproduct from the effluent stream, an $\mathrm{HCl}$ scrubber was installed and tied-in to a furnace capable of heating to $800^{\circ} \mathrm{C}$ (the "low-fire" step), under Subtask 3.5. With the use of this new slurry, virtually no delaminations were apparent within the wall of the filter elements, fewer candles were damaged during mandrel removal, and better adhesion between adjacent yarns was been observed.

With regard to the membrane quality issue, Westinghouse's filtration efficiency test exposed the filter to only one ash penetration challenge, and showed no penetration to the inner body. The test protocol assumed that once a smooth ash layer was built up, it would adhere to the filter surface, and thereby take over future surface filtration. The thin, loose ash cakes on PRD-66 filters after Tidd exposures, however, brought that assumption into question. The Westinghouse test protocol also assumed that if ash penetrated the surface membrane, it would immediately show up on the inner diameter. Based on the hypothesis described above, the standards by which a membrane is deemed "acceptable" needed to be changed, at least where PRD-66 was concerned. The PRD-66 membrane would need to function as a much better ash barrier to minimize the risk of "divots" and to reduce the pressure buildup caused by accumulated entrained ash.

For the "baseline" filter, the leakage through the outer membrane appeared to occur through tiny gaps between the adjacent yarns of the "wound-on" membrane. Apparently, the alumina slurry coating on the fiberglass yarns did not consistently bridge the gaps between the yarns and an incomplete membrane formed. Furthermore, gaps appear to occur more frequently, 
where the membrane yarn covers a primary crossover point in the pattern of the support winding underneath.

Several options were evaluated for improving the quality of the membrane layer

1. a different filament winding pattern for the body

2. a double outer membrane

3. a different type of membrane yarn

4. additions to the membrane layer

To test the efficiency of such alternate membrane technologies, 2-inch test segments were exposed to the Particle Infiltration Test (PIT), described in Figure 17. All samples were examined in transmitted light for areas of ash penetration; a subjective scale of appearance, ranking from " 1 " (many large wide-spread infiltration areas) to "10" (no detectable areas of ash infiltration), was established. Several specimens of each candidate were generally prepared to evaluate reproducibility.

Another critical aspect of the evaluation was to quantify the backpressure of the experimental membranes. 8-inch specimens of the promising candidates were prepared. Many of the membranes, which were studied, had excellent PIT ratings, but resulted in backpressure above Westinghouse's acceptable limits. For 8-inch long units, tested at $5 \mathrm{scfm}$, the target was 10 inches water gauge. In some cases, new membranes were evaluated for permeability first; only acceptable candidates were leak tested in the PIT.

Almost one hundred different combinations of the variables mentioned above were tested. A statistical evaluation was not feasible, however, certain conclusions, concerning the effectiveness of the varying approaches, could be drawn.

Filament Winding Patterns. It had been observed that many gaps occurred where the membrane yarn covered a primary crossover point in the pattern of the support winding underneath. Attempts were made to alter the winding pattern of the body to create a smoother surface on which to wind the membrane yarn. Although initial changes looked promising, each new pattern was very time-consuming to model and implement, and produced only marginal improvements. Consequently, no changes were made to the "baseline" winding pattern.

Double Outer Wound Membrane. The addition of a second layer of membrane yarn, on top of the first, was evaluated using a variety of slurry types, yarn spacings, and yarn types. 
Although several combinations produced units with good PIT ratings, the backpressure exceeded the 10-iwg target. Consequently, the winding of two outer layers of membrane yarn was not incorporated into the "baseline" product.

Different Membrane Yarns. PRD-66 filters use fiberglass yarn, which is available with varying amounts of twist. It was hoped that by using a less twisted grade, the yarn would lie flatter on the surface of the filter body, the edges of adjacent yarns could overlap, and the gaps could be eliminated. Although this concept was demonstrated, the untwisted yarn was very difficult to work with and broke frequently during winding. Consequently, no yarn changes were incorporated into the "baseline" product.

Additions to the Wound Membrane Layer. Initially, the focus was on filling the gaps between adjacent membrane yarns with ceramic fibers, ceramic particles or ceramic precursors. Although many combinations were effective filters, they had poor permeability (high backpressure). By using these filler materials INSTEAD OF a hoop-wound membrane yarn, permeabilities that are more reasonable were achieved. The contours on the surface of the filter body, however, made reproducibility difficult. The most effective solution was to apply a hoopwound membrane with intentional gaps between adjacent yarns and then fill those gaps with a material that gave appropriate filtration and backpressure. This membrane modification was incorporated into the "baseline" product and was commonly referred to as a "combination" membrane.

In summary, to correct the problem of the leaky membrane, identified in Subtask 3.4, the most promising approach chosen for further study was a membrane comprised of a "hoop-wound" yarn with a ceramic filler material in-between adjacent windings. To improve the interlaminar strength of the support body underneath, the filter would be fabricated using the modified slurry composition. 


\subsection{Development of High Efficiency Membranes}

\subsubsection{Membrane Development for the Outside Diameter (OD) Surface (Subtask 3.1)}

To facilitate this addition of a ceramic filler material, a new pattern was chosen for the 'hoop-wound' yarns allowing broader spacing between adjacent yarns. Instead of relying on the microcracks in the alumina slurry to provide adequate filtration, a more controlled material would be used to fill in the gaps and provide a uniform porosity. The approximate relationship of this new spacing to the original membrane spacing is depicted in Figure 21 and Figure 22, showing the additional filler material between the 'wound-on' yarns, and the additional membrane area created in this process.

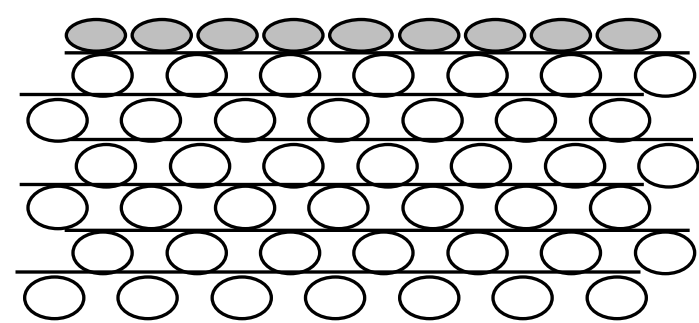

Figure 21 - Original wound membrane (wall cross-section).

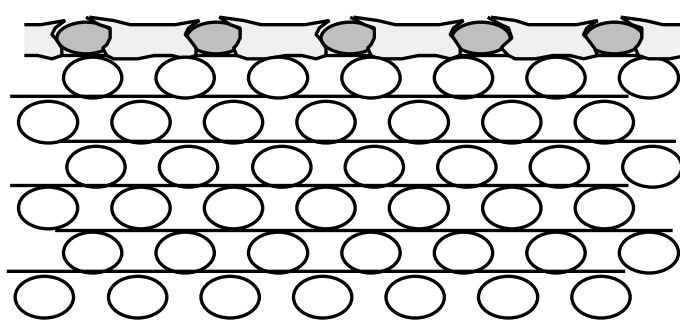

Figure 22 - Membrane with added filler (wall cross-section)

The composition of the filler material was varied over as wide a range of options and a variety of application techniques were attempted. Some of the variables evaluated included:

1. Particulate Alumina: 220-grit, 320-grit, 400-grit, 100-grit tabular alumina, fumed alumina

2. Ceramic Precursors: aluminum chlorohydrate, colloidal alumina, colloidal silica

3. Application Technique: brushing, hand-rubbing, spraying, immersion, squeegeeing

4. State of Filter Body: unfired, partially-fired, fully-fired

The criteria used for comparison consisted of "ease of application", "uniformity", "reproducibility", "adherence", "permeability", and "filtration efficiency". Candidate membranes were selected for further evaluation only if they scored a PIT rating >"9" after 25 exposure cycles. Figure 23 illustrates a unit with a rating of " 10 ". The specimen pictured in Figure 18 would be representative of a rating of " 3 ". 


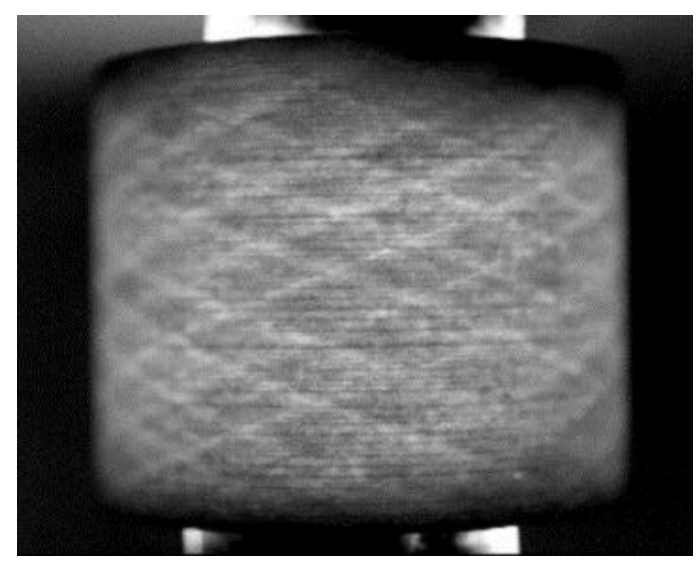

Figure 23 - Modified membrane with PIT rating of "10".

After assessment of a large number of filter segments, another advantage of transmitted light inspection became readily apparent. Any defects, which appeared as ash-infiltrated darkened areas in the PIT tested samples, had also been apparent in the untested samples when examined by transmitted light. Although small membrane defects on the order of $100-200 \mu$ diameter were not readily apparent on routine visual inspection (Figure 24), they became visible as intensely bright points of light in transmitted light inspection (Figure 25). Further, these defects were detectable in the filters prior to firing, allowing for the application of additional membrane filler before the final ceramic conversion firing.

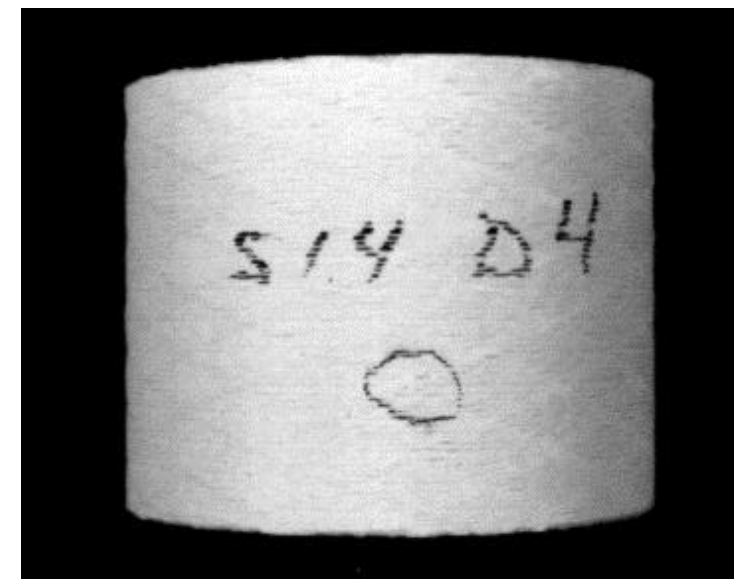

Figure 24 - Hole in membrane, undetectable under direct light.

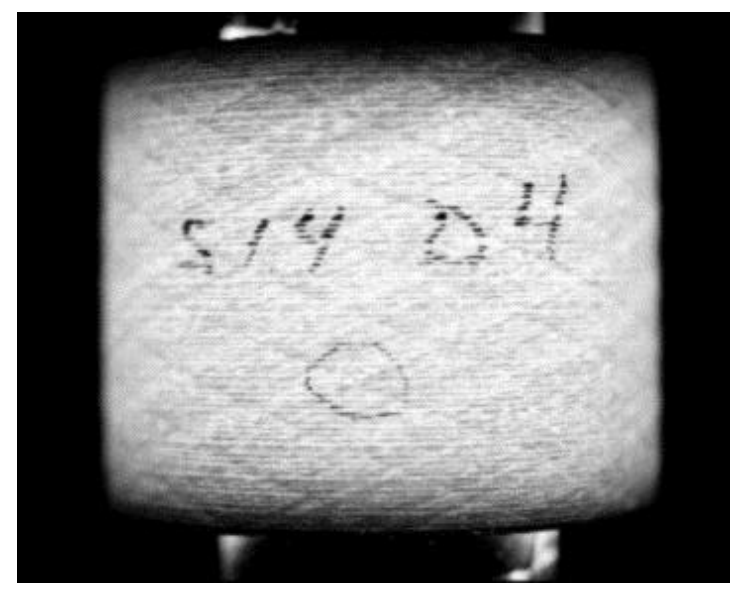

Figure 25 - Hole in membrane, detectable under transmitted light.

Controlled testing of specimens with membrane defects was conducted. Each sample was examined in transmitted light prior to firing, some pinholes were filled with additional material, and some were left open. Specimens were subjected to 25 PIT cycles. All sites where ash penetration occurred, during PIT exposure, had been easily located prior to firing. None of the 
filled pinholes showed signs of leakage. No additional defects developed during the final ceramic conversion firing. Figure 26 shows the result of testing a defective segment where a pinhole, detected prior to firing, was allowed to remain.

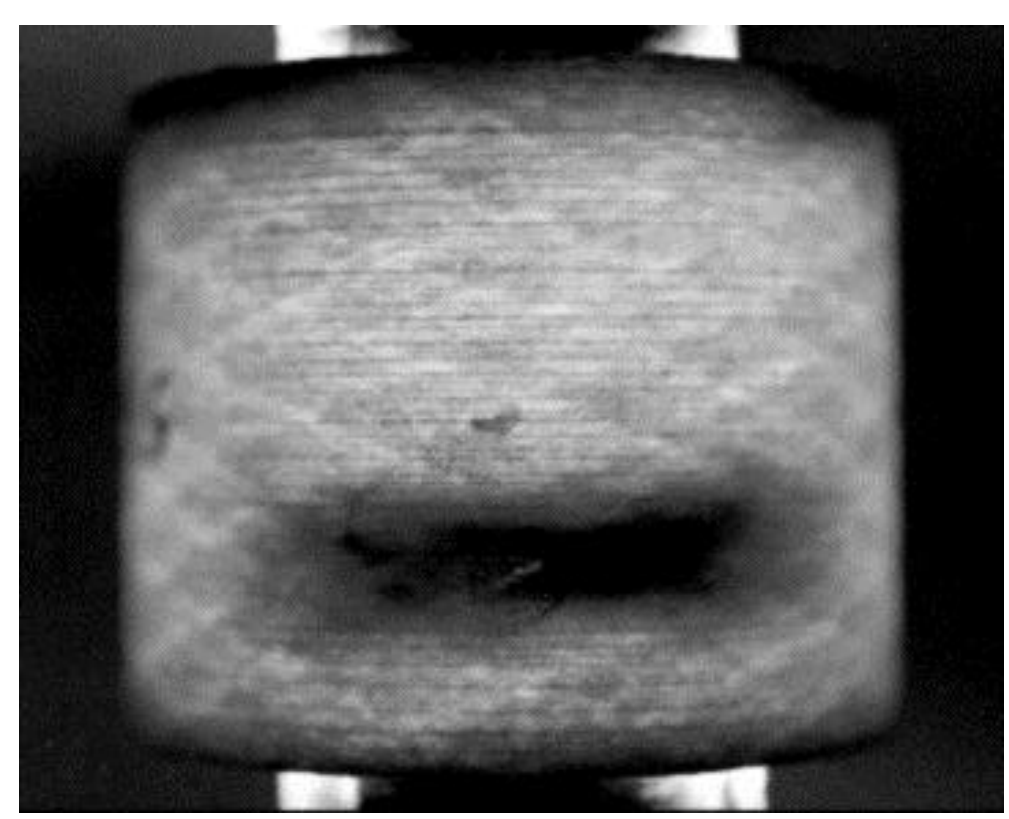

Figure 26 - Hole in membrane after 25 PIT cycles, viewed in transmitted light.

This defect was virtually undetectable when examined in direct light, but immediately obvious in transmitted light. This test and defect elimination procedure was added to DLC's standard manufacturing protocol for $100 \%$ of PRD-66 production filters.

From the many candidate membranes tested, two variants were selected for further evaluation. PRD-66M and PRD-66C were selected for their excellent, but different, combinations of filtration performance and flow resistance characteristics. Both of these membrane candidates were processed into full size filter elements for testing at the Westinghouse HTHP facility. PRD$66 \mathrm{M}$ has a mean pore size for filtration of about $10.5 \mu$ (Figure 27) with flow resistance comparable to the close wound membrane filters. Flow resistance of 1.5 -meter filters was tested both before and after HTHP testing, as shown in Figure 28. 


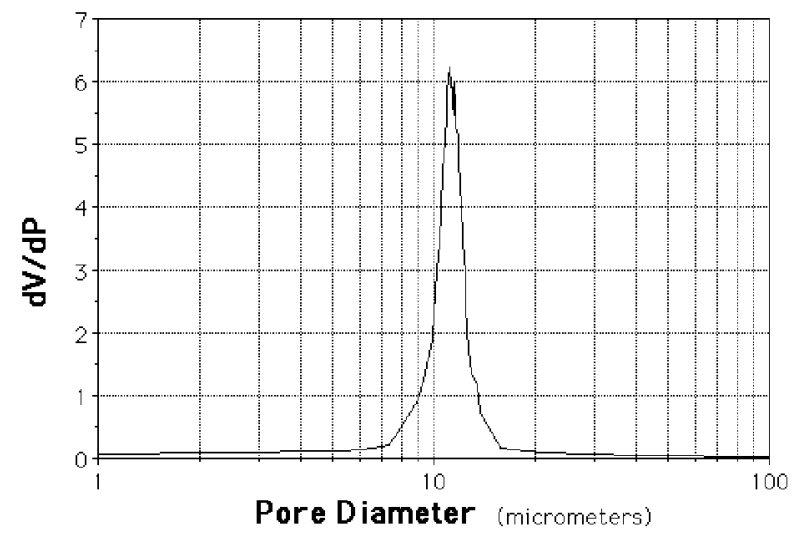

Figure 27 - PRD-66M membrane, measured pore distribution.

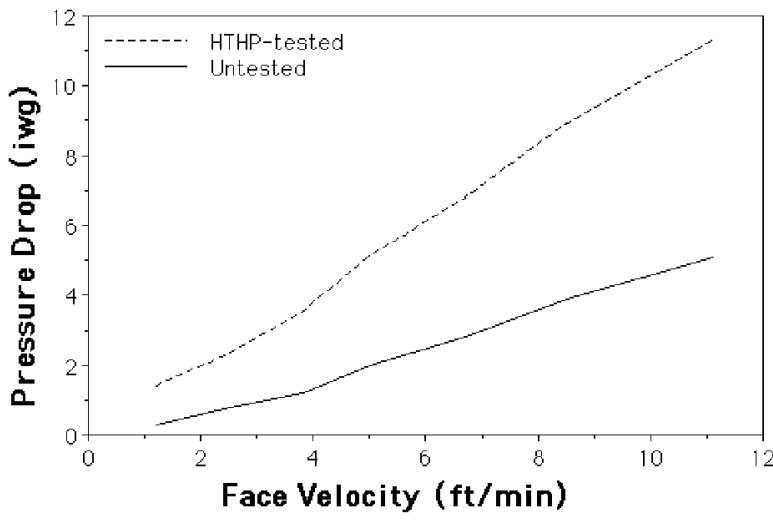

Figure 28 - PRD-66M flow resistance for 1.5-meter candles.

The second membrane candidate, PRD-66C, was chosen because of its unusually low flow resistance in combination with excellent filtration performance. With a mean pore size of about $25 \mu$ (Figure 29) its flow resistance is less than half that of filters with PRD-66M membranes (Figure 30).

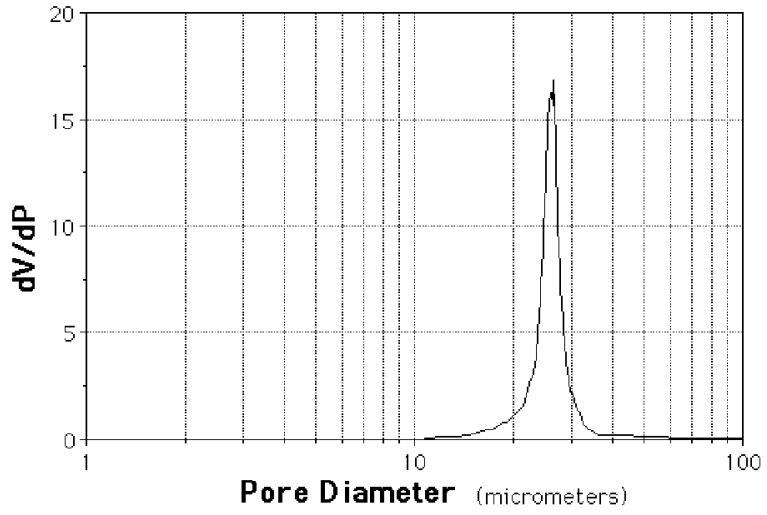

Figure 29 - PRD-66C membrane, measured pore distribution.

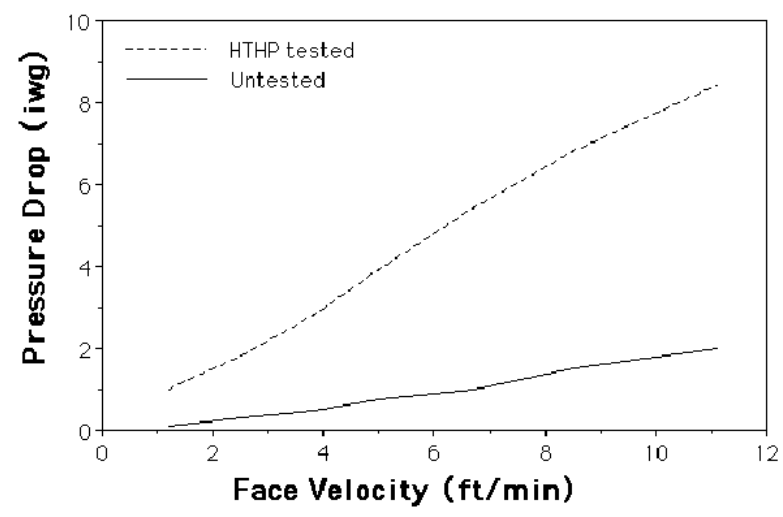

Figure 30 - PRD-66C flow resistance for 1.5-meter candles.

Both membrane types are considered viable candidates for future commercialization. The choice of which to use would depend on system requirements. Further refinements of the membrane composition are detailed in Section 3.5.3.2 "Variables". 


\subsubsection{Membrane Development for the Inside Diameter (ID) Surface (Subtask 3.1.1)}

This task was a continuation of the Dual Membrane Candle Filter concept described in Section 3.2.2 of this report, but it was conducted near the end of this contract. The modifications described in this section were not used in the production of any filters described in Sections 3.5, $3.6,3.7$, and 3.8 of this report.

By using some of the later PRD-66 innovations, the primary goal was to develop a membrane layer that would protect the inside diameter of the support wall from accumulating ash on "clean side". Westinghouse referred to this problem as "backside blinding", which can be caused by an incident such as a broken filter. An event like this occurred in a PFBC field trial in Karhula, Finland (Section 3.6.2.1). A PRD-66 filter that was effected is shown in Figure 48, Page 65. Because the inside diameter surface is so open, the ash penetrated over $1 \mathrm{~mm}$ into the support wall (Figure 50, Page 67). A membrane on this surface would, theoretically, be more easily cleaned.

Before the development of an appropriate inside diameter (ID) membrane could commence, a test was necessary which evaluated the integrity of that surface. A particle infiltration test that would expose only the inside diameter of a filter sample to coal ash was required. Although it would be similar to the original PIT device (Figure 17, page 26), the engineering challenges were very different. Potential designs were evaluated based on:

- the availability of components

- the assurance of a good seal

- the ease of loading and unloading the specimen

- the ease of removing the ash cake between each test cycles

A schematic of the device is shown in Figure 31. To conduct the test, a 4.5" long candle filter sample is mounted in the tester. Approximately $100 \mathrm{cc}$ of ash is placed into the screw-on cap at the base of the unit. The vacuum is turned on and the device is manually inverted to disperse the ash. After 5 seconds, the device is set up-right and the vacuum turned off. The cleaning brush is then pushed down, and pulled back, through the center of the filter specimen. Another ash exposure is then conducted and the specimen ID cleaned. After 25 exposure cycles, the specimen is evaluated using transmitted light. 


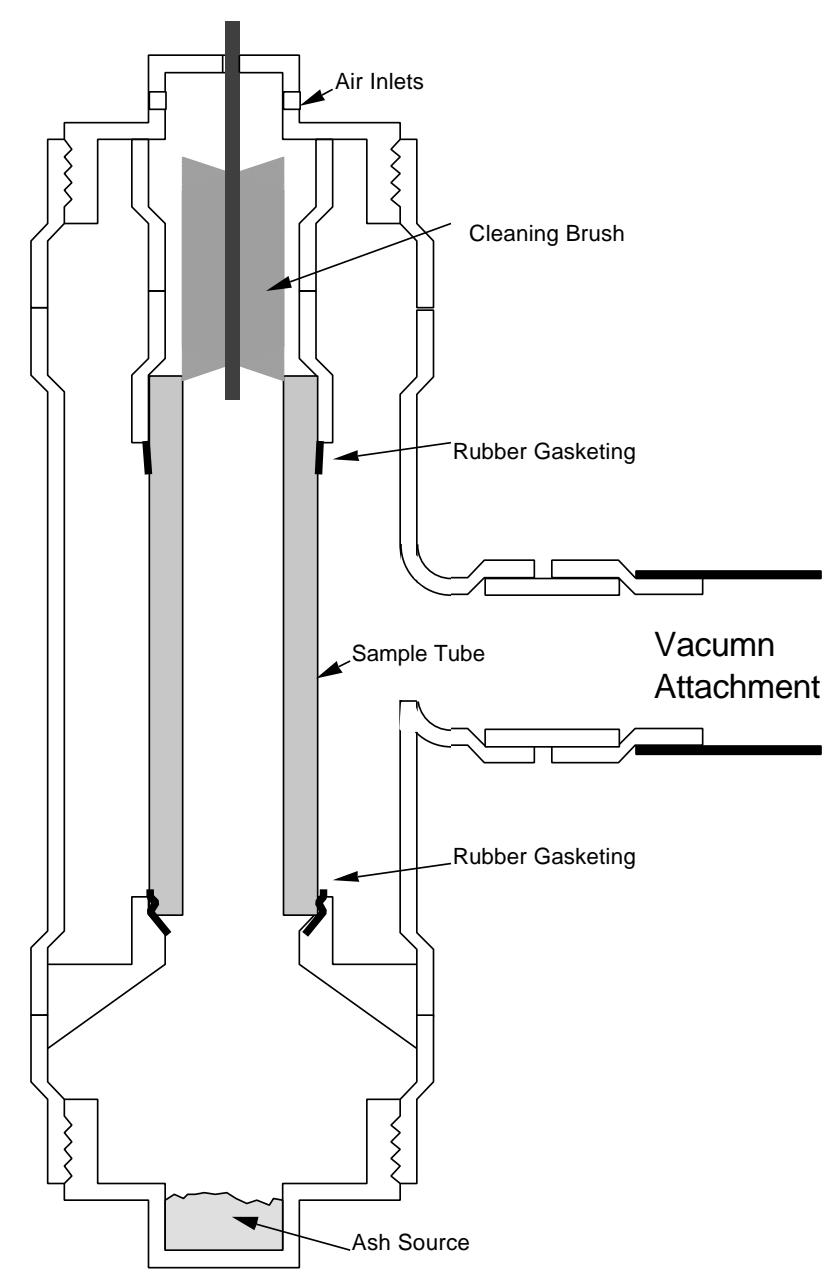

Figure 31 - Particle Infiltration Test device for ID membranes

To demonstrate the functionality of this device, a old candle filter with both an ID and OD membrane (Section 3.2.2) was tested, then examined using transmitted light. The areas of ash penetration can be seen as dark blotches within the filter wall, as shown in Figure 32.

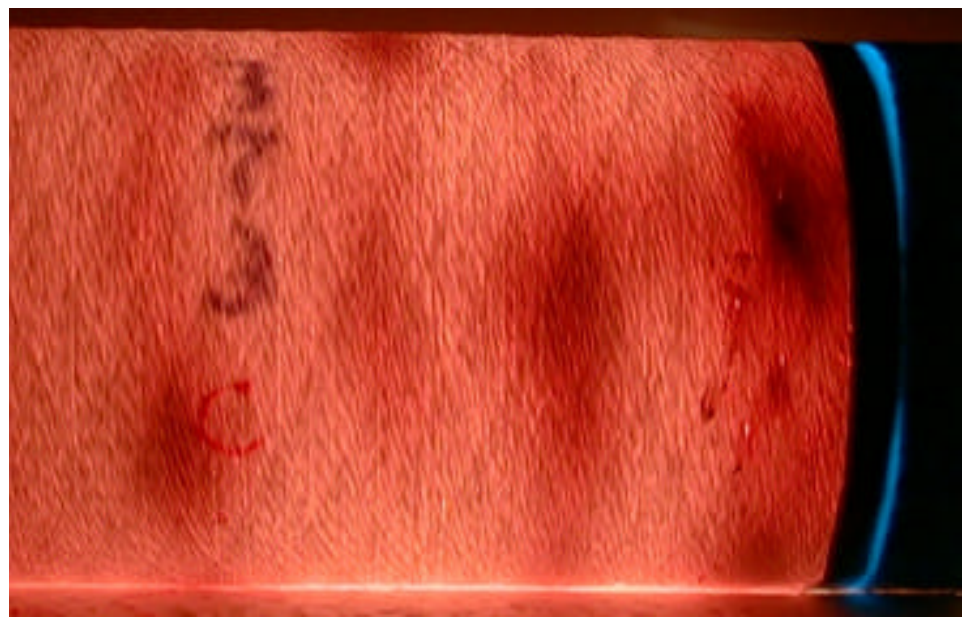

Figure 32 - ID P.I.T. evaluation of original "hoop-only dual membrane" filter (1995) 
One issue that had to be addressed in this task was the elimination of the relatively minor damage commonly inflicted on the inside surface of the filter, during the application of the membrane-filler on the outside diameter. For several of the inside membrane application techniques being considered, this type of damage would severely compromise the integrity of the membrane surface.

The standard method for applying the alumina particulate to the outside surface is to slide the low-fired candle filter onto a mandrel, as shown in Figure 33. As with the original mandrel, the support-pin at the tip protrudes through the hole in the tip of the candle filter. This mandrel is then supported at both ends and rotated, while the membrane filler is rubbed into the surface. The most common problem is that, when the mandrel is removed from the low-fired filter, the supportpin at the tip snags several strands of yarn from the inside diameter and pulls them out.

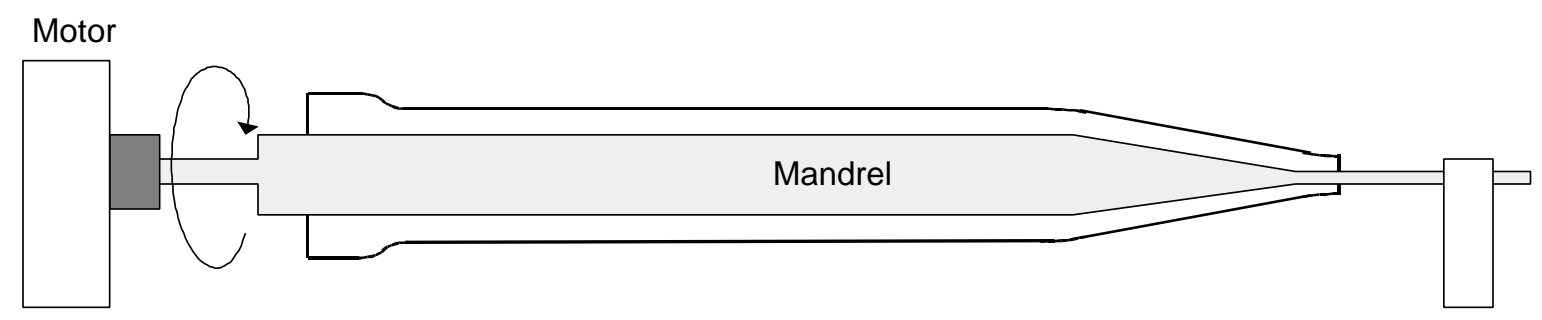

Figure 33 - Standard filter support scheme during membrane-filler application.

A redesign of this equipment was conducted, as illustrated in Figure 34. Instead of sliding the low-fired filter onto a typical mandrel, the filter is slid over a cantilevered shaft, which is rotated directly by a motor. When tested with standard filters (outside diameter membrane only), the tearing of the yarns along the inside surface was significantly reduced by not eliminated. Any additional modifications would have to wait until filters with ID membranes were actually available.

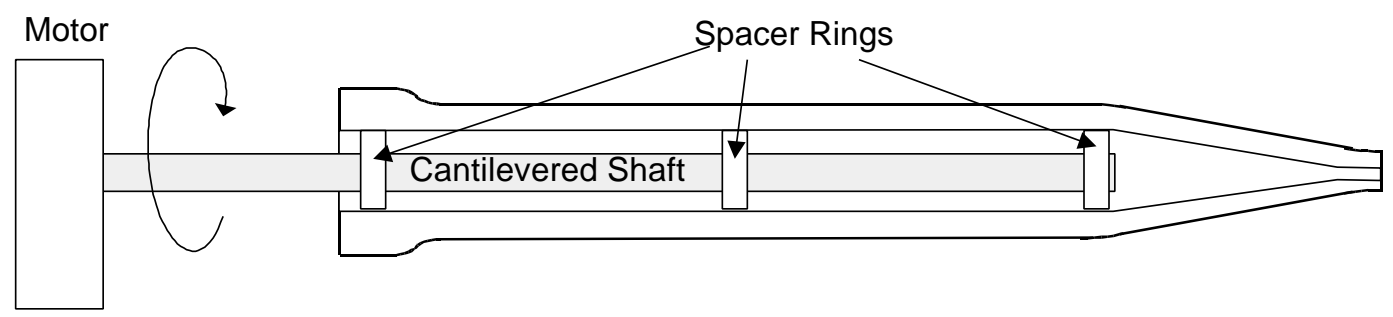

Figure 34 - Cantilevered filter support for membrane-filler application. 
The development of the actual membrane layer differed from the original work done several years earlier (Section 3.2.2) in several respects. New materials and techniques were now being used which made an ID membrane appear to be more feasible:

- Chlorhydrol@-based alumina slurry (Section 3.3.3.5)

- alumina particulate membrane fillers (Section 3.4)

- less damaging mandrel removal techniques (Section 3.5.5)

- a technique to detect if the membrane leaked (Section 3.4.2)

The development of the ID membrane focused on using the alumina particulate membrane. The challenge was to develop an application method capable of producing an ID membrane with the following characteristics:

- acceptable backpressure (by Westinghouse standards)

- uniform backpressure from end-to-end of the filter element

- reproducible results from candle-to-candle

- good membrane adhesion

Several techniques were investigated for creating the particulate membrane layer in the early stages of the filter winding process. If the filter could have been fabricated with the inside membrane already in place, it would have dramatically simplified later processing. Unfortunately, a uniform coating of the alumina particulate suspension was very difficult to achieve. Samples were fabricated and tested, which demonstrated its effectiveness, but only on short filter segments. The most promising technique, along these lines, involved spraying the particulate. After winding a single layer of yarn in a "hoop" fashion onto a standard filter mandrel, a high-pressure air gun was used to spray the a particulate covering over the "hoop-layer". It was difficult, however, to get a suitably thick coating. Before this approach could be evaluated further a different type of spray gun would be required and spraying would need to be conducted in a dust-controlled enclosure.

The most feasible technique required the application of the membrane to the inside surface of the filter after the filter had been low-fired. A series of experiments were conducted to coat the inside diameter by a "slip casting" technique. One particular trial gave very good results. A Type$\mathrm{C}$ particulate membrane suspension (slightly more fluid than that used for the outside membrane), was poured into the interior of 8-10" long pieces of low-fired filters. A stopper had been placed in one end of the specimen and the suspension was poured out after approximately one minute. The resulting cake was $2-3 \mathrm{~mm}$ thick along the ID wall. While the cake was still wet, a rubber stopper just slightly smaller than the ID of the tube was pushed through the sample to scrape away the 
majority of the excess. The resulting surface as very smooth, but the thickness was not uniform. After several days of drying, an attempt was made to remove more of the excess by using a rotating tool with very fine sandpaper.

The general appearance of the membrane was very good, but apparent pinholes were noticed when the surface was visually examined. The most promising candidate was tested in the "Inside Diameter, Particle Infiltration Tester" (ID P.I.T.) and examined with transmitted light as shown in Figure 35. No indication of ash penetration could be found.. After performing the ID P.I.T., the sample was cut up and the sites of the pinholes were reexamined. The "apparent" pinholes were actually bubbles, which were only open on the ID side of the membrane. Based on these findings, later work focused on the feasibility of de-airing the suspension to reduce bubbles.

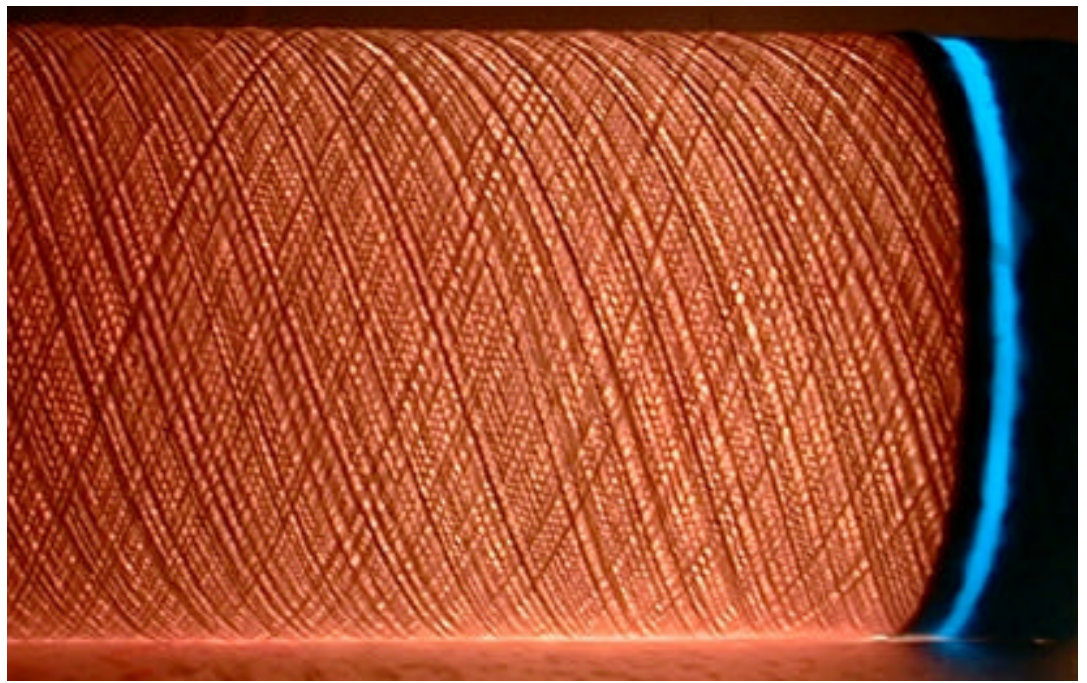

Figure 35 - ID P.I.T. evaluation of "slip-cast ID membrane" filter (no membrane on OD)

In addition to having a suitable membrane suspension, it was critical to find a technique for scraping the excess membrane material from the inside diameter of a full-length filter. With the sample tubes, the excess was removed by simply sliding a stiff, rubber stopper through the tube shorlty after the majority of the suspension had been poured out. A full size candle, or any specimen with one closed end, poses the following challenges:

- The scraper or squeegee must be pulled out, not pushed through.

- The scraper must be positioned in the filter before pouring in the suspension.

- The scraper must fit tightly along the inside surface, but not damage the yarn along the inside diameter during insertion.

- The suspension must be able to flow past the scraper, to fill the conical-shaped closed end (thorough removal of the material from this section is not considered critical). 
The most feasible design to accomplish this task is shown in Figure 36. It is meant to be use by first inserting it into the filter. The alumina particular suspension is then added and poured out two minutes later. By twisting the nut at the end of device, the rubber disc near the tip is compressed. As the disc expands, makes contact with the inside diameter of the filter. The entire assembly would then be pulled out.
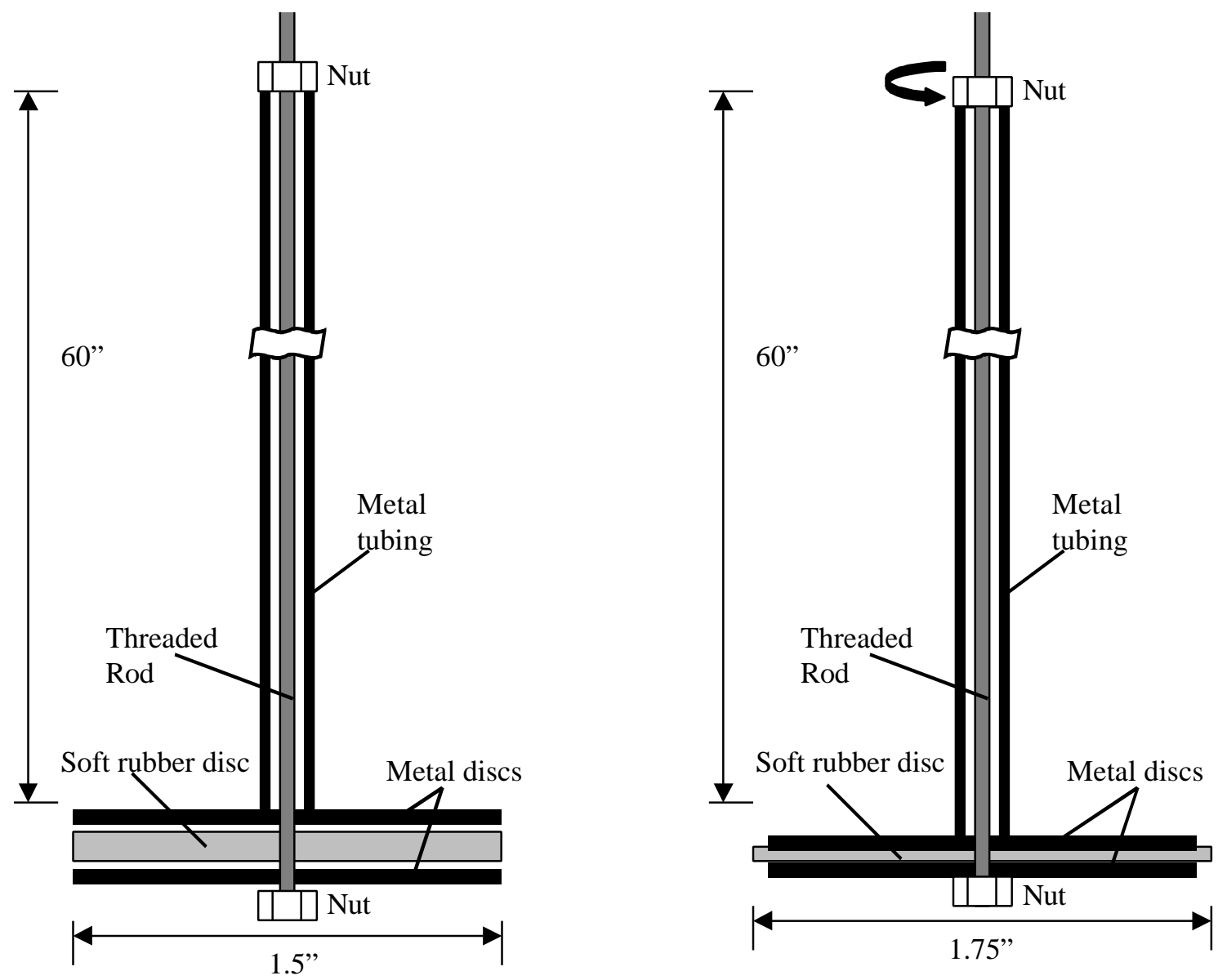

Figure 36 Device for removing excess membrane from the inside surface of a filter.

At the conclusion of this contract, different types of rubber discs had been evaluated, but none gave as smooth a surface as desired. 


\subsection{Manufacturing Hot Gas Filters (Task 4)}

The focus of Task 4 was to lay the foundation for the repeat manufacturing of PRD-66 Hot Gas Candle Filters. The effort was divided into six areas: raw materials plan, process instrumentation, process variable experiments, process capability demonstration, equipment analysis and improvement, and evaluation of long-term degradation.

\subsubsection{Raw Materials Plan (Subtask 4.1)}

Discussions were held with DLC's quality organization to align the PRD-66 product with the company's overall quality plan. Copies of DLC's documentation requirements for raw materials specifications are detailed in Appendix 2. DLC will develop specifications for all raw ingredients necessary to the production of PRD-66 Hot Gas Candle Filters and require Certificates of Conformance (COC) and/or Certificates of Analysis (COA) with each shipment which document conformance of the incoming raw ingredients with specifications. Raw material suppliers were contacted about our requirements and were very cooperative in meeting them.

\subsubsection{Process Instrumentation (Subtask 4.2)}

The goal of this effort was to identify any critical equipment used to perform in-process measurements and establish methods to assure the level of calibration necessary to maintain process control.

The most important instruments used in fabricating hot gas candle filters are the electronic balances. Several balances, with different accuracy ranges, are utilized at different stages of the process. When winding candle filters, the bobbins of feed yarn (see Figure 1) are positioned on balances, which have a maximum load of 2,000 grams (+/-0.1 gram). The amount of yarn that is used in the preform is determined by the net change in the indicated weight of the feed bobbin. This weight, when compared to the weight of the actual candle, is used to calculate the amount of alumina picked-up when the yarn is dipped into the alumina slurry (see Figure 1). Adequate pickup is necessary to insure the strength of the product.

A larger capacity balance, with a +/- 5-gram readout, is necessary for weighing the raw materials that comprise the alumina slurry. This balance is also used for the weighing of the 
candle filters; although, a more accurate balance with +/- 1-gram accuracy would be preferable for this purpose, if one was available.

All balances are calibrated annually in accordance with NIST HB44, ISO 10012-1 and ANSI/NCSL Z540 requirements. During the period of this contract, balances were calibrated several times and all were found to be within acceptable tolerances.

The only other critical instrument used in the PRD-66 process is a Brookfield viscometer. This devise measures the viscosity (resistance-to-flow) of liquids. Viscosity standards were purchased from Brookfield with known viscosities similar to that of the alumina slurry used in the PRD-66 process. No measurable deviations from calibration were observed throughout the period of this contract.

Although the viscosity of the slurry is critical in a broad sense, experiments performed during Task 4.3 (3.5.3 - Process Variables Experiments) indicate that variations as high as 50\% from nominal have no impact on the process. For this reason, the viscometer does not require routine calibration checks. It is critical, however, to ensure that the settings on the instrument are always appropriate for the spindle being used. An incorrect setting, for example, could lead one to believe that the viscosity is $100 \mathrm{cps}$, when if fact it is 1,000 cps. For this reason, use of this equipment is restricted to the PRD-66 project staff, and is used only for alumina slurries having similar viscosities.

\subsubsection{Process Variables Experiments (Subtask 4.3)}

The focus of this subtask was to identify critical process parameters and vary them systematically to learn their effect on the product. In order to identify which variables the process was most sensitive to, ranges were chosen to encompass and exceed the existing specifications. If there was minimal sensitivity at the values tested, the existing specifications would be deemed acceptable. If sensitivity was detected, a more thorough evaluation would be conducted in order to define appropriate parameter limits.

The standard conditions for winding the fiberglass yarn included the use of the improved slurry composition, which had improved strength in the dry-state and better interlaminar adhesion, see Section 3.3.3.5. 


\subsubsection{Variables Impacting the Support Winding}

The variables studied for their impact on the winding of the filter support were slurry viscosity, winding speed and atmospheric humidity. The ranges investigated were chosen based on current process capability to control them, see Table 5.

\begin{tabular}{|lcc|}
\hline Variable & Lower Limit & Upper Limit \\
\hline Winding Speed & $-22 \%$ & $+22 \%$ \\
Alumina Slurry Viscosity & $-50 \%$ & $+50 \%$ \\
Relative Humidity & $20 \%$ & $80 \%$ \\
\hline
\end{tabular}

Table 5 - Process variables investigated for winding filter support.

Candle filter support structures were wound, without flanges and without membranes. Winding was terminated when the weight of the fiber wound reached 1100 grams. Any unusual events that occurred were noted during the course of each run. After overnight air drying, tubes were each cut into eight, 8 "long sections and the two end pieces retained as scrap. All portions were fired to $700^{\circ} \mathrm{C}$ ("low-fired"), held for one hour and allowed to cool to room temperature. All portions were weighed and measured, then high-fired to approximately $1400^{\circ} \mathrm{C}$. Alumina pickup was calculated based on the low-fired weights and the known weight of the fiberglass yarn and high-fired materials were flow tested and inspected for delaminations. A summary of the results is depicted in Table 6 .

\begin{tabular}{|l|l|l|}
\hline Variable & Lower Limit & Upper Limit \\
\hline Winding Speed & very slight increase in diameter & no detectable effect \\
\hline Alumina Slurry Viscosity & $\begin{array}{l}\text { statistically significant decrease } \\
\text { in alumina pickup }\end{array}$ & no detectable effect \\
\hline Relative Humidity & slight increase in diameter & slight decrease in diameter \\
\hline
\end{tabular}

Table 6 - Observed impact of process changes on support winding.

The lower and upper limits, which were tested, are all outside the normal process limits, yet, only the use of an "alumina slurry with half of the normal viscosity" resulted in a statistically significant change. No statistically significant variations in the product occurred within the nominal viscosity range. None of the other changes were statistically significant, suggesting that the normal process control limits are adequate for the reproducibility of PRD-66 candle filters.

Experiments were also conducted to determine the effect of "process interruptions during the winding operation" on process quality. The most critical type of interruption is an unattended 
yarn break during winding. To simulate this type of problem, the winding was intentionally stopped approximately half way into an otherwise routine winding run. The package was allowed to sit for approximately 15 minutes while still rotating, although a five-minute interruption would be more typical of current process norms. This experiment was conducted under a range of humidity conditions. Winding was restarted following standard procedures, and stopped at the target diameter. After the tube was dried overnight, it was cut, low-fired, weighed, and measured as described earlier; the specimens were then high-fired through a standard cycle to approximately $1400^{\circ} \mathrm{C}$.

The only sample impacted by the winding interruption was the unit wound at the lowest humidity condition, which was outside of the normal operating range. When the completely fired material was cut, and the cross-section examined, a slight delamination could be discerned at approximately the mid-way point in wall, closely corresponding to the point at which the winding had been interrupted. Apparently, process interruptions of up to 15 minutes can be tolerated without adversely affecting the product, except in humidity conditions which are generally outside the normal range. Besides the resulting improvement in product yields, the insensitivity to interruptions will allow the use of "short bobbins" of fiberglass yarn. Standard bobbins of S-2 yarn typically have about $25 \%$ more yarn than is actually required for winding one candle filter. To stop the winding, and string-up a new bobbin of yarn usually takes approximately three minutes. The ability to do this without jeopardizing product quality will lead to less wasted yarn and lower costs.

An additional variable, added to this experiment, was the impact of fiberglass yarn "twisted" by a different company. Owens-Corning FiberGlas (supplier of S-2 glass yarn) decided that they would no longer directly supply yarn that is "twisted" in a wide assortment of configurations, including that required by this process. Two alternate sources of this twisted yarn were identified; only one, however, was reasonably priced. Three candle filters were fabricated from yarn twisted by the Varflex Corporation (Owens-Corning is still the sole manufacturer of the S-2 glass filaments). The run information was compared to the database that had been generated in earlier portions of this task. Evaluations were conducted on " alumina pickup", diameter growth rates, frequency of yarn breaks, and integrity of the overall structure. The twisted yarn from Varflex appeared to be either equivalent or superior to the original material in all tests. DLC's current inventory of yarn (purchased from Owens-Corning) is adequate to complete the fabrication of candles required for this program, but future purchases will be made from Varflex. 


\subsubsection{Variables Impacting the Membrane}

As discussed in Section 3.4, several variables were identified as being critical to the formation of a satisfactory membrane for the PRD-66 Hot Gas Candle Filter. Under Task 4.3, extensive tests were conducted to identify a membrane-filler formulation that would consistently yield low backpressure units with good filtration. The variables explored included:

1. 4 different solid-to-liquid suspension ratios

2. applying the particulate material to low-fired or high-fired candles

3. 2 different particle or grit sizes of alumina

4. 2 different levels of a fusible binder addition

Evaluations of items " 1 " and "2" were based on subjective comparisons of the ease of preparation and application of the filler material. The preferable solid-to-liquid ratio (2:1) was an aqueous suspension with a consistency similar to very, smooth peanut butter. More consistent results were achieved by applying this filler material to the surface of low-fired candles. Samples prepared in this manner with the medium-grit membrane, however, frequently developed extremely fine cracks in the membrane during the final firing, visible only with intense scrutiny using transmitted light. These cracks were so fine that no Tidd ash penetrated after 25 PIT cycles. Evaluations of items " 3 " and " 4 " were conducted in a more quantitative fashion, as shown in Table 7.

Both the "coarse" and "medium" grit alumina particulate are capable of producing membranes with a PIT rating of "10". The two grit sizes, however, had different ashcake release characteristics in the PIT evaluation, with the ash being more adherent to the coarse-grit membrane. In the Karhula field trial, this type of candle exhibited the formation of a traditional "conditioned ash cake layer". Tests of the original "baseline" candle in Tidd did not form such a layer; the repeated exposure of the imperfect membrane surface, after backpulsing, was thought to have contributed to the entrainment of ash in the filter wall. 


\section{PRD-66 Membrane Variations and Reproducibility}

\begin{tabular}{|c|c|c|c|c|c|}
\hline $\begin{array}{c}\text { 8" Unit } \\
\text { ID }\end{array}$ & $\begin{array}{c}\text { Full Length } \\
\text { Candle ID\# }\end{array}$ & $\begin{array}{c}\text { Membrane } \\
\text { Grit Size }\end{array}$ & $\begin{array}{c}\text { wt\% } \\
\text { Binder }\end{array}$ & $\begin{array}{c}\text { Weight of } \\
\text { Added Membrane }\end{array}$ & $\begin{array}{c}\text { Backpressure } \\
\text { in-wg @ 5scfm }\end{array}$ \\
\hline \hline 1 & 553 & Medium & $5 \%$ & 9.03 & 5.6 \\
\hline 2 & 553 & Medium & $5 \%$ & 8.50 & 7.0 \\
\hline 3 & 553 & Medium & $5 \%$ & 8.55 & 5.6 \\
\hline 4 & 553 & Medium & $5 \%$ & 8.94 & 5.5 \\
\hline 5 & 553 & Coarse & $5 \%$ & 8.14 & 2.2 \\
\hline 6 & 553 & Coarse & $5 \%$ & 7.23 & 5.2 \\
\hline 7 & 553 & Coarse & $5 \%$ & 8.53 & 3.4 \\
\hline 8 & 534 & Coarse & $5 \%$ & 7.67 & 2.6 \\
\hline 9 & 534 & Coarse & $5 \%$ & 7.38 & 1.8 \\
\hline 10 & 534 & Medium & $5 \%$ & 7.85 & 4.0 \\
\hline 11 & 555 & Medium & $5 \%$ & 7.22 & 5.4 \\
\hline 12 & 555 & Medium & $5 \%$ & 7.85 & 5.6 \\
\hline 13 & 534 & Coarse & $5 \%$ & 5.73 & 3.0 \\
\hline 14 & 555 & Coarse & $5 \%$ & 5.83 & 1.9 \\
\hline 15 & 555 & Coarse & $10 \%$ & - & 2.1 \\
\hline 16 & 555 & Coarse & $10 \%$ & - & 3.2 \\
\hline 17 & 555 & Coarse & $10 \%$ & - & 3.1 \\
\hline 18 & 555 & Coarse & $10 \%$ & - & 1.9 \\
\hline
\end{tabular}

Table 7 - Impact of grit-size and binder content on backpressure.

The data shown in Table 7 was also used to evaluate the impact of applying reproducible amounts of the particulate membrane. A correlation of the weight of the membrane filler and the backpressure was plotted in Figure 37. In general, the exact amount of the added membrane filler did not directly effect backpressure, at the quantities being used; in severe cases, however, excess material has been observed to crack during the high-fire step. 


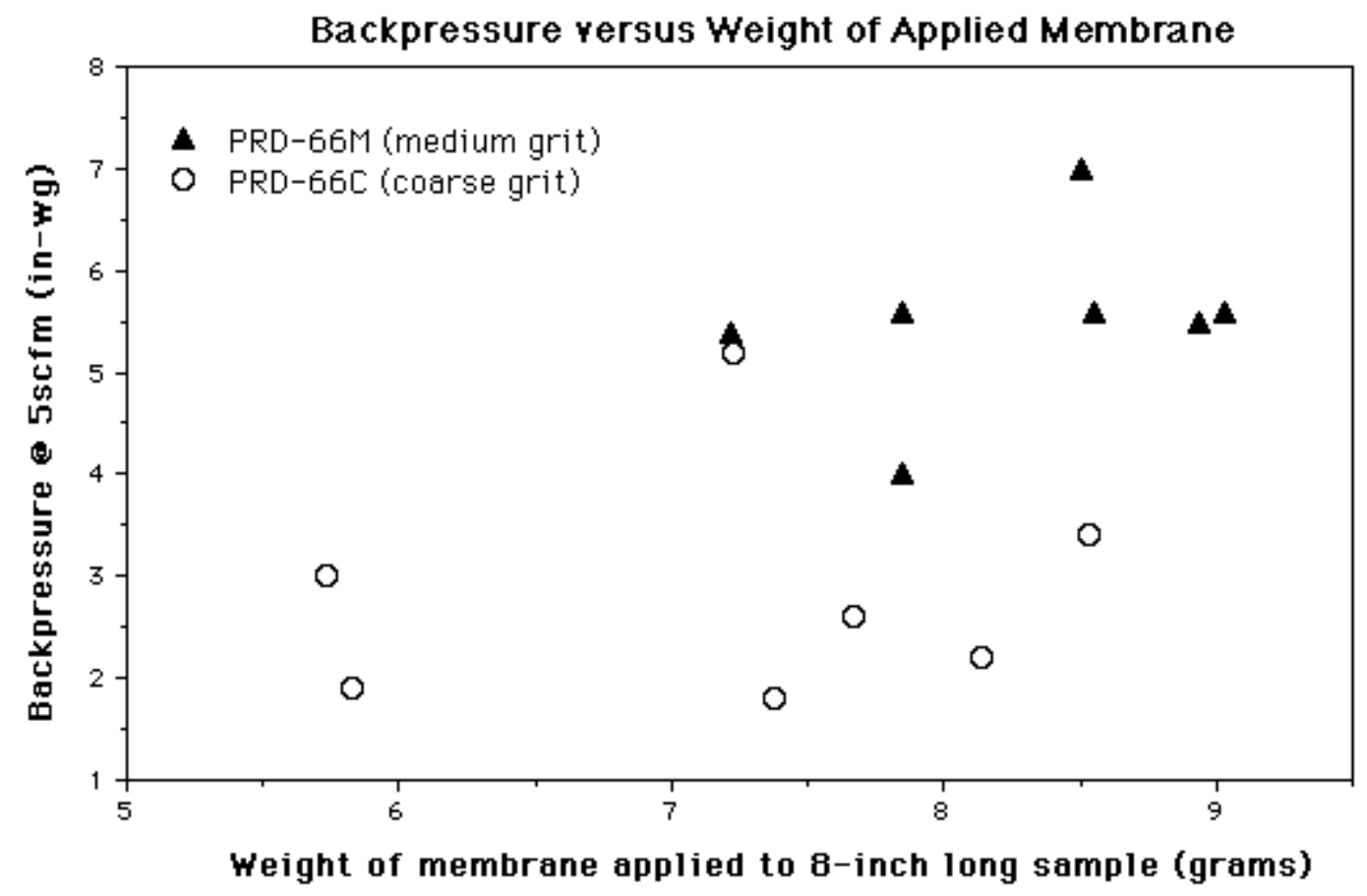

Figure 37 - Impact of membrane weight and type on backpressure.

Backpressure also appeared to be relatively unrelated to the binder content in the larger grit size composition, see Table 8. The higher level of fusible binder addition seemed to be preferable for the coarse-grit filler; the resultant material adhered better to the surface of the candle, as observed in the repeated brushing involved in the PIT evaluation. This level of fusible binder was not necessary with the medium-grit filler-material, probably because the higher surface area of the finer particles sintered more readily. Fortunately, a higher level of fusible binder did not seem to significantly impact backpressure.

\begin{tabular}{|c|c|c|c|}
\hline \multicolumn{4}{|c|}{ Backpressure of Coarse Samples Only vs. Binder Content } \\
\hline $\begin{array}{c}\text { Binder } \\
\text { Content }\end{array}$ & No. of samples & \multicolumn{2}{|c|}{ Backpressure @ 5scfm (in-wg) } \\
\hline & & Average & Std. Dev. \\
\hline $5 \%$ & $\mathrm{n}=7$ & 2.9 & 1.2 \\
\hline $10 \%$ & $\mathrm{n}=4$ & 2.6 & 0.7 \\
\hline
\end{tabular}

Table 8 - Impact of binder content on backpressure of PRD-66C.

Based on the experiment described above the membrane formulations chosen for further evaluations were: "medium grit with 5\% binder" and "coarse grit with $10 \%$ binder" 
In addition to the membrane experiments described above, an evaluation was conducted to determine the effectiveness of filling "pin holes" in the unfired membrane. Eighteen low-fired, 8 " filter segments were coated with either the "coarse" or "medium" grit membranes. After the membrane dried, each unit was checked with transmitted light for "pin holes". Additional membrane filler was then applied to those areas and marked with a high-temperature marking pencil, to make later identification possible. After high-firing, all specimens were examined again. All patched areas appeared completely sealed and no additional "pin holes" developed.

Earlier in this section, mention was made of the formation of extremely fine membrane cracks after high-firing the PRD-66M candle filters. The reason for their occurrence was not determined. In general, these flaws were only visible using transmitted light, and then, only if you knew exactly what to look for. If significant amounts of excess filler-material remained on the surface, the cracks were more severe and visible to the eye under normal lighting conditions. Preparation of multiple samples, from virtually identical tubes, has yielded significant information. Only the membrane made with the medium-grit, or finer, alumina particulate exhibits the problem, under normal conditions. The problem is minimized by using lower levels of the fusible binder addition, but not eliminated. When several 8" samples, from the same candle, were prepared in the same way with the medium-grit filler, and fired side-by-side, only one sample in the batch had cracks. As noted earlier, a specimen with a crack was PIT-tested with Tidd ash; the ash was trapped in the membrane and did not penetrate into the support wall. It is unknown whether or not this condition jeopardizes the successful operation of the candle. Aggressive investigation was discontinued due to the time constraint of providing filters to Westinghouse for testing. The best-known formulations and application methods would be used. General and specific information, with regard to handling of the candles, placement within the furnace, etc., would be monitored and correlations would be sought with any incidence of cracking. 


\subsubsection{Process Capability Demonstration (Subtask 4.4)}

The focus of this subtask was to produce three batches of candle filters, according to the specifications required by the Westinghouse Advanced Particulate Filtration (APF) System, as shown in Figure 38. Each batch consisted of ten candles, manufactured under identical conditions. Before beginning each batch, critical components of the process equipment was inspected. Where feasible, new parts were put into service and process changes were incorporated to improve the product quality and process yields. An evaluation was conducted on all measurable features of the filters to assess controllability and product uniformity. Significant aspects of the process, which effected final yields, were identified. Eight of the first-quality candles were used for high-temperature, high-pressure (HTHP) testing at Westinghouse Science and Technology Center (see Section 3.6.1). Twelve of the first-quality candles were field tested at the Foster Wheeler 10 MWt PCFBC facility in Karhula, Finland (see Section 3.6.2).

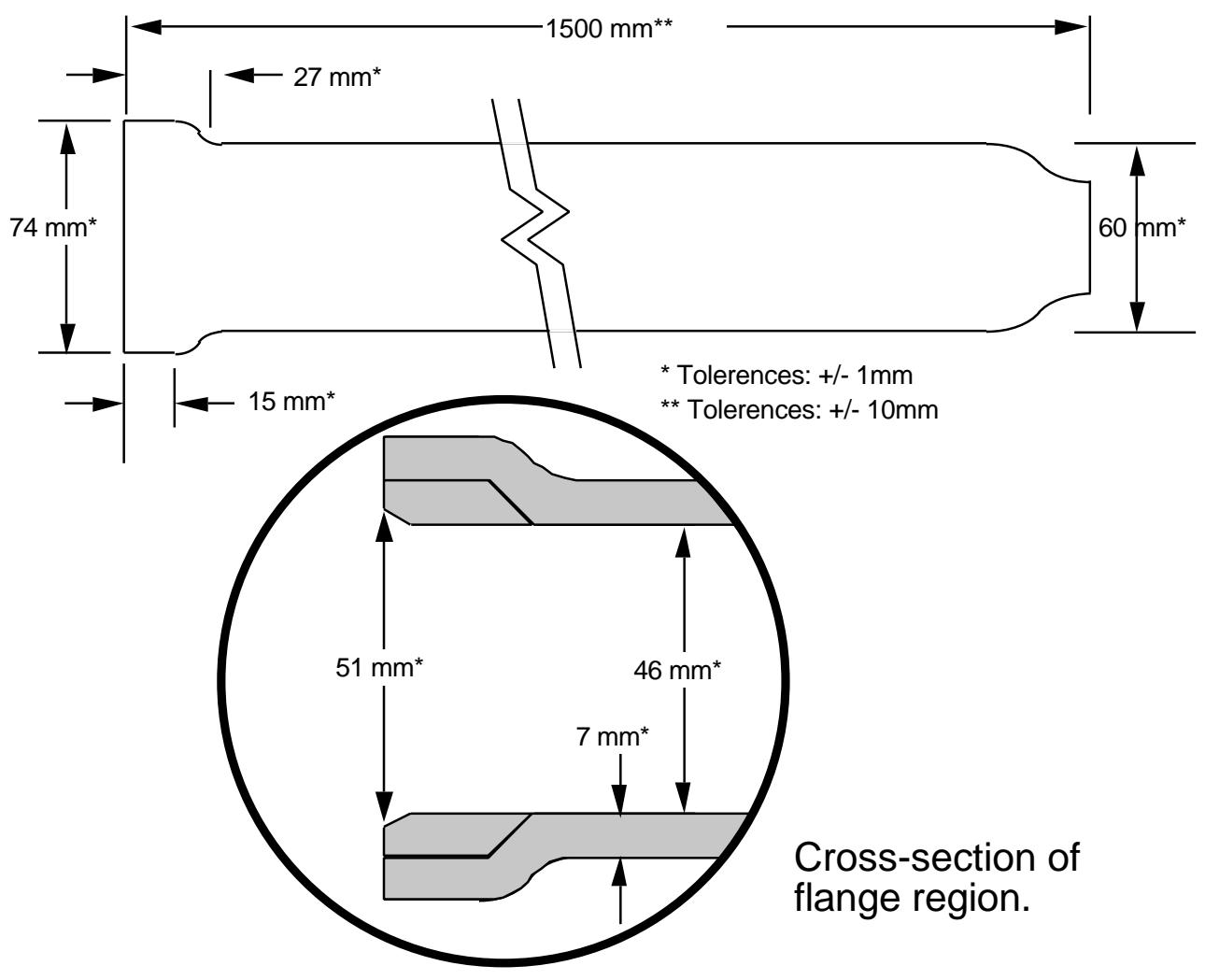

Figure 38 - PRD-66 Candle Filter dimensions

During this capability study, twenty-one good candles were produced, out of a possible 30 , or $70 \%$ yield. Table 9 gives a detailed evaluation of all elements fabricated. Table 10 summarizes the data into the three, ten-unit runs, which were conducted. 


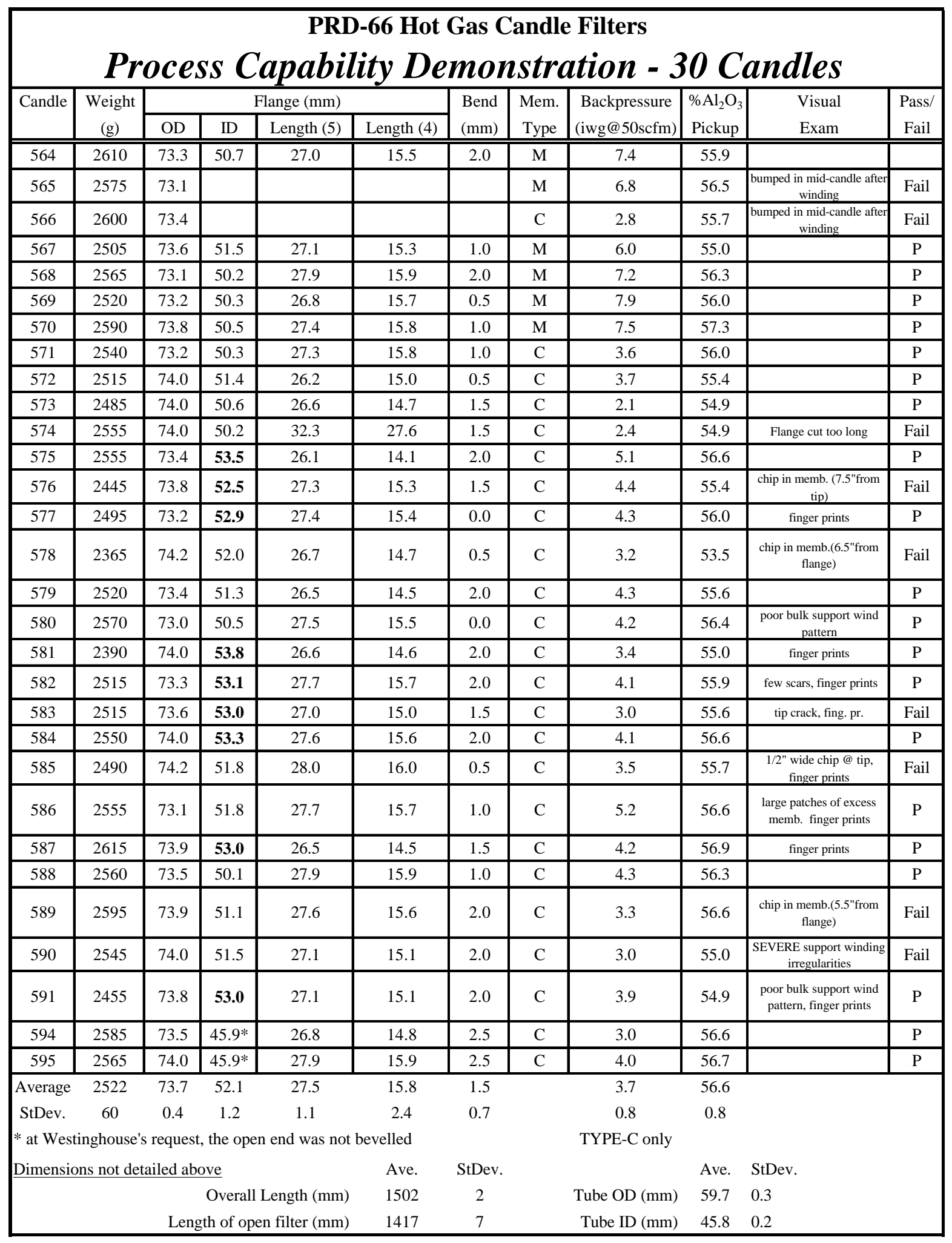

Table 9 - Process Capability Demonstration 


\begin{tabular}{|c|c|c|c|c|c|c|c|c|c|c|c|c|}
\hline & $P r$ & $C$ & & PRD & $\begin{array}{l}-66 \mathrm{H} \\
\text { ility }\end{array}$ & $\begin{array}{c}\text { ot Gas } \\
\text { oem }\end{array}$ & $\begin{array}{l}\text { Cand } \\
\text { Onst }\end{array}$ & $\begin{array}{l}\text { le Filte } \\
\text { ratio }\end{array}$ & rs & $u m$ & mary & \\
\hline & \multirow{2}{*}{$\begin{array}{c}\text { Weight } \\
(\mathrm{g})\end{array}$} & \multicolumn{2}{|c|}{ Length $(\mathrm{mm})$} & \multicolumn{2}{|c|}{ Tube (mm) } & \multicolumn{4}{|c|}{ Flange (mm) } & \multirow{2}{*}{$\begin{array}{l}\text { Bend } \\
(\mathrm{mm})\end{array}$} & \multirow{2}{*}{\begin{tabular}{|c|} 
Backpressure \\
(iwg@50scfm)
\end{tabular}} & \multirow{2}{*}{$\begin{array}{c}\% \mathrm{~A} 12 \mathrm{O} 3 \\
\text { Pickup }\end{array}$} \\
\hline & & \begin{tabular}{l|l} 
Overall \\
\end{tabular} & Open* & OD & ID & OD & ID & $\mathrm{L}(5)$ & $\mathrm{L}(4)$ & & & \\
\hline \multicolumn{10}{|c|}{ TOTAL (30 candles) } & \multicolumn{3}{|c|}{ TYPE-C only } \\
\hline Average & 2522 & 1502 & 1417 & \begin{tabular}{l|l}
59.7 \\
\end{tabular} & 45.8 & 73.7 & 52.1 & 27.5 & 15.8 & 1.5 & 3.7 & 56.6 \\
\hline StDev. & 60 & 2 & 7 & 0.3 & 0.2 & 0.4 & 1.2 & 1.1 & 2.4 & 0.7 & 0.8 & 0.8 \\
\hline Target & & 1500 & & 60.0 & 46.0 & 74.0 & 51.0 & 27.0 & 15.0 & 0.0 & & \\
\hline Spec. & & $+/-10$ & & $+/-1.0$ & $+/-1.0$ & $+/-1.0$ & $+/-1.0$ & $+/-1.0$ & $+/-1.0$ & $<3.0$ & & \\
\hline \multicolumn{13}{|c|}{ RUN 1 (10 candles) } \\
\hline Average & 2551 & 1502 & 1405 & 59.6 & 46.0 & 73.5 & 50.7 & 27.0 & 15.5 & 1.2 & 3.1 & 55.9 \\
\hline StDev. & 43 & 3 & 4 & 0.3 & 0.0 & 0.4 & 0.5 & 0.5 & 0.4 & 0.6 & 0.8 & 0.7 \\
\hline $\operatorname{Max}$ & 2610 & 1505 & 1411 & 60.1 & 46.0 & 74.0 & 51.5 & 27.9 & 15.9 & 2.0 & 3.7 & 57.3 \\
\hline Min & 2485 & 1499 & 1397 & 59.2 & 46.0 & 73.1 & 50.2 & 26.2 & 14.7 & 0.5 & 2.1 & 54.9 \\
\hline \multicolumn{13}{|c|}{ RUN 2 ( 10 candles) } \\
\hline Average & 2493 & 1501 & 1417 & 59.7 & 45.7 & 73.6 & 52.3 & 27.5 & 16.2 & 1.3 & 3.8 & 55.5 \\
\hline StDev. & 70 & 3 & 6 & 0.3 & 0.2 & 0.4 & 1.2 & 1.8 & 4.0 & 0.8 & 0.8 & 0.9 \\
\hline $\operatorname{Max}$ & 2570 & 1508 & 1426 & 60.0 & 46.0 & 74.2 & 53.8 & 32.3 & 27.6 & 2.0 & 5.1 & 56.6 \\
\hline Min & 2365 & 1499 & 1405 & 59.3 & 45.5 & 73.0 & 50.2 & 26.1 & 14.1 & 0.0 & 2.4 & 53.5 \\
\hline \multicolumn{13}{|c|}{ RUN 3 (10 candles) } \\
\hline Average & 2552 & 1502 & 1418 & 59.8 & 45.9 & 73.8 & 52.0 & 27.4 & 15.4 & 1.7 & 3.9 & 56.2 \\
\hline StDev. & 65 & 3 & 99 & 0.3 & 0.2 & 0.4 & 1.2 & 1.8 & 4.0 & 0.7 & 1.5 & 1.0 \\
\hline $\operatorname{Max}$ & 2615 & 1505 & 1426 & 60.5 & 46.2 & 74.2 & 53.3 & 28.0 & 16.0 & 2.5 & 5.2 & 56.9 \\
\hline $\operatorname{Min}$ & 2455 & 1500 & 1410 & 59.2 & 45.7 & 73.1 & \begin{tabular}{|l|}
50.1 \\
\end{tabular} & 26.5 & 14.5 & 0.5 & 3.0 & 54.9 \\
\hline
\end{tabular}

\section{Table 10 - Process Capability Summary}

Seven elements were rejected as a result of physical damage incurred during some stage of the processing. One element was rejected because the flange was out-of-spec (too long). One element was rejected for a poor quality membrane. Although, the "inside edge diameters" of nine flanges were out-of-spec, Westinghouse felt confident that their holder assembly could accommodate them, so they were not rejected.

The physical damage to the filter elements appeared to have two distinct sources. The first occasion for significant damage to occur was during the transfer of the developing candle from the bulk support winder to the membrane hoop winder (while it was still soft, damp and easily dented). Any obstructions on the equipment or between the winders increased the risk of damage. When two candles were dented, it was immediate and obvious.

Of more serious concern were several filter elements which each had a single chip (approximately 1/8"-1/4" long and 1/16"- 1/8" wide) in the membrane, discovered during final inspection. After final firing, the damaged areas "puckered" and the membrane easily flaked off 
when rubbed. Based on historical observations, the damage probably occurred while the candle was in the unfired or low-fired state. Possible causes include excessively tight gripping during a difficult mandrel removal or contact of the membrane with an inadequately padded area of the storage cart. In either case, damage would not have been apparent prior to the final high temperature firing.

Examining the standard deviation of the data, most of the features were within $3 \%$ of the average and within the acceptable range established by the Westinghouse protocols. The inside diameter of the flange and the length of the flange, however, were much more difficult to keep inspec:

Inside diameter of the flange. The inside diameter of the flange was out-of-spec on $30 \%$ of the candles fabricated. The open end of each candle was finished-off by grinding a bevel on the inside edge, such that, the finished edge of the ID was $51 \mathrm{~mm}+/-1 \mathrm{~mm}$. The hand grinding technique, which was employed to create this bevel, was not adequately reproducible; machining was not a viable economic option. The original reason for the grinding was that the inside edge of the flange was occasional too friable, resulting in an irregular surface. Throughout the course of this program, however, with the adoption of the Chlorhydrol@-containing alumina slurry (see Section 3.3.3.5), the inside edge became much denser than with the original composition. The added step of grinding this area no longer appeared to be necessary; both DLC and Westinghouse agreed to eliminate this feature in future production runs.

Length of the flange. The data does not wholly reflect the difficulty encountered in meeting the required tolerances. Because the outside contour of the PRD-66 flange has no distinct edges, defining the precise location for cutting is not simple. It was also difficult to establish whether the flange was "in-spec" or "out-of-spec". All measurements were taken based on how the flange aligned with a plastic tool having a similar contour. Several candles which seemed to be slightly too long were hand-ground into spec. No problems were encountered, by Westinghouse or Foster Wheeler, mounting any of these candles for field trials. Eventually, better measurement techniques and better-defined specifications will be needed.

The data collected during the process capability run (see Table 9) indicated that the alumina matrix pickup varied from $53.5 \%$ to $57 \%$. A possible link between diametrical compressive strength and alumina matrix pickup was investigated. 1" wide o-rings were cut from the candle with the $53.5 \%$ pickup and o-ring diametrical compressive tests were performed. The 
strength values were within the range of all measurements previously taken. During the course of Task 5 additional tests will be conducted on the candle with the lowest matrix, pickup to see if any impact on strength can be observed. Furthermore, any finished candle having a damaged portion, making it unsuitable for field use, will be cut up into 1" o-rings and tested in order to define the nominal strength range of PRD-66 filter elements. This information will be essential in determining if field-exposed elements are any stronger or weaker than the as-manufactured material.

An important objective of this task was to gain a better understanding of the process economics of manufacturing PRD-66 Hot Gas Filters. The most dramatic finding was that the utilization of the winding equipment was well below expectations due to the high level of equipment maintenance required. While some problems were anticipated as a result of wear, the biggest difficulties encountered were inherent in the basic winder design. Many of the features that make this devise very versatile compromise its reliability under routine operating conditions. A simpler winder, designed specifically for PRD-66 candle filters, would require significantly less time, labor, and materials to maintain. 


\subsubsection{Equipment Analysis and Improvement (Subtask 4.5)}

During “Task 4.4 - Process Capability Demonstration”, described in the previous section, an analysis of the rate of wear of critical components was conducted. Attention was initially focused on surfaces that were in contact with abrasive slurry-coated yarn and the moving components of the winder itself. As part of "Task 4.5 - Equipment Analysis and Improvement", the feasibility and cost of making improvements was be evaluated and changes made where appropriate.

The first issue addressed was an increase in the frequency with which the slurry-coated yarn would break during the winding process. Breaks usually occurred when the traverse changed direction and the yarn needed to slide from one side of the guide to the other. The most obvious reason for this problem was that the alumina guide would develop grooves on either side, because of abrasion from the particulate alumina in the slurry. The deeper the grooves became the more likely the yarn was to break when the traverse changed direction. Two potentially more abrasion-resistant materials were evaluated: metal-matrix composite and polycrystalline diamond. The metal-matrix composite material turned out to be even more susceptible to abrasion. The polycrystalline diamond guide was never actually tried; it was prohibitively expensive to achieve a sufficiently rounded surface that would not cut the yarn. Since neither material offered any advantages over the high purity alumina, the alumina guide was changed out more frequently to keep yarn breaks to a minimum.

During this investigation, however, another reason for yarn breaks was observed. The yarn would most frequently break during the first 20 minutes of winding, when the guide changed direction at the tip-end of the mandrel. The mandrel on which the PRD-66 filter element was wound had a hemispherical shape at the tip end, going from $45 \mathrm{~mm}$ down to $6 \mathrm{~mm}$ in diameter in approximately 1" of length, as shown in Figure 39.

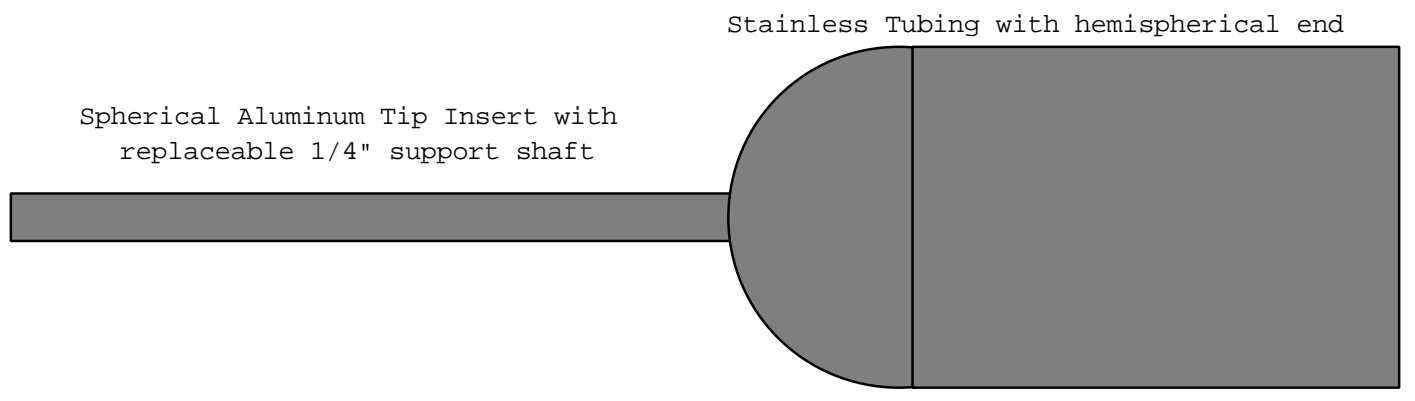

Figure 39 - Tip of original steel mandrel 
When the yarn wound down to the narrow support shaft, the speed at which it was being removed from the yarn bobbin (see Figure 1, page 6) would slow dramatically; as the guide carried it back up to the $45 \mathrm{~mm}$ tubing, the yarn would be "tugged" suddenly, often breaking the yarn. As a layer of yarn accumulated on the shaft, thus increasing its diameter, this became less of a problem. During the first twenty minutes of winding, however, constant supervision and slower winding speeds were required. To address this problem a design change was made, to use a conical-shaped tip instead of a hemispherical one; this change was instituted along with other changes intended to create more easily removable mandrels. After the changes were implemented, the frequency of breaks dropped dramatically.

Another problem this task sought to address was the difficulty with which the wound filter was removed from the mandrel. In several cases, damage to the candle could result, which was not always easily detected until much later in processing (see Section 3.5.4). Several combinations of steel tubing, plastic tubing and rubber were evaluated. The mandrel chosen for future manufacturing use was made from readily available sizes of tubing, with a rubber conical tip, and could easily be removed from the filter after spending about 30 minutes in a freezer. Because of the use of standard tubing sizes, the filters were approximately $1 \mathrm{~mm}$ smaller in the inside diameter. Sample candles were send to Westinghouse for their feedback. Westinghouse had to modify the design of their "fail-safe devise" to accommodate the inside diameter change.

Another issue addressed in this task was the inadequacy of the procedure and tools used to cut the scrap ends from the dried candle filters. The standard procedure required the use of a razor knife, while rotating the candle (while still on the mandrel). After the finished candles were checked for perpendicularity, however, many flanges required hand grinding in order to meet the specification. A new concept was evaluated involving the use of a rotating, circular blade, while rotating the candle/mandrel. A silicon carbide blade and a diamond wafering blade were both tested. The diamond blade was the most effective and was used with later candles made in the "Process Capability Demonstration". The need for hand finishing of the final filters was reduced.

A major equipment issue involved the repair of DLC's 15-ft long X 4-ft wide high-fire furnace. The deterioration of the roof insulation over the previous six years led to detectable temperature non-uniformities along the length. The PRD-66 Hot Gas Filter Program was only user of this equipment, so the repairs were conducted under this program.

Some of these modifications were implemented during the "Process Capability Demonstration", and most were put in place by the start "Task 5 - Manufacturing 50 Candles". 


\subsubsection{Redesign of Prototype Winding Equipment (Subtask 4.7)}

The intent of this work was to reevaluate the winding operations which produced PRD-66 candle filters during "Task 5: Manufacturing 50 Filters" (Section 3.8), then redesign the winding hardware to demonstrate concepts that would assist in the development of future commercial equipment.

The winding equipment used for the fabrication of hot gas candle filters was designed for research purposes and was able to create a wide variety of cylindrical structures in small quantities. In the course of Task 4 and Task 5 , several minor, budgeted changes were made to maximize the output of these winders. Under constant routine operation, significantly more equipment maintenance was required than had been anticipated, and significantly lower yields resulted from equipment malfunctions during the production of the filters. The current prototype winder was clearly not well-suited for high production volumes.

Another manufacturing challenge arose because the winding of the membrane was performed on a separate piece of equipment. There was only one "hoop winder" to handle the output of two "support winders". Since the "hoop winding" takes approximately one-tenth the time of the support winding, this did not seem to be a problem. Difficulties arose during Task 5, however, when the timing of units coming off the two "support winders" conflicted with the availability of the one "hoop winder". An additional reason to combine these two features was the risk of damage during the transfer. At this stage in its development, the filter body is very soft and easily deformed.

Efforts in this task were focussed, therefore, on those features that enhanced equipment reliability and product reproducibility, and incorporated the "hoop" and "support" winding into one piece of equipment. The specific research hardware, and related designs, were understood to be proprietary to the contractor (ACI) because they were simply variations of original handling methods developed prior to the initiation of this contract.

The initial modifications were completed over a four-month period. The primary changes included: fewer gears and belts to wear our, more stable bearings and supports, and the addition of the "hoop" winding mechanism. During the initial runs, several winding interruptions occurred while winding the support yarn, but its diamond-pattern, looked virtually perfect. In addition, the mechanism that winds the hoop membrane produced a yarn pattern significantly better that the 
original "hoop winder". As shown in Figure 40 and Figure 41, the wraps of membrane yarn were much more evenly spaced using the modified winder. More uniform membranes should significantly improve backpressure reproducibility.

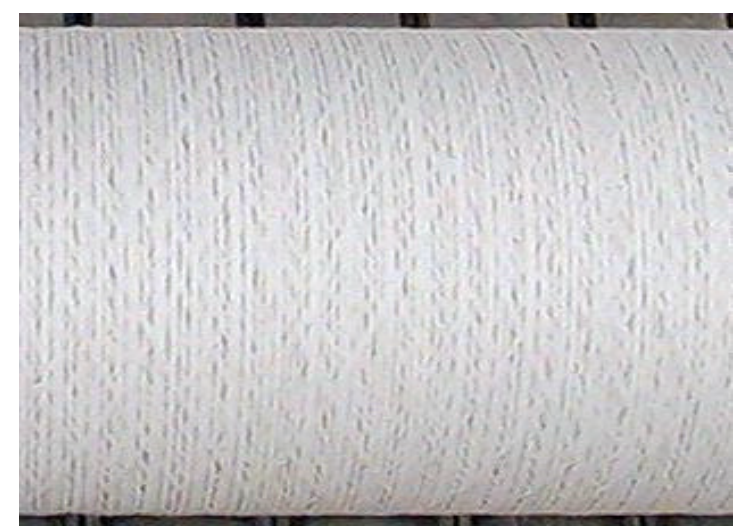

Figure 40 - Original hoop membrane

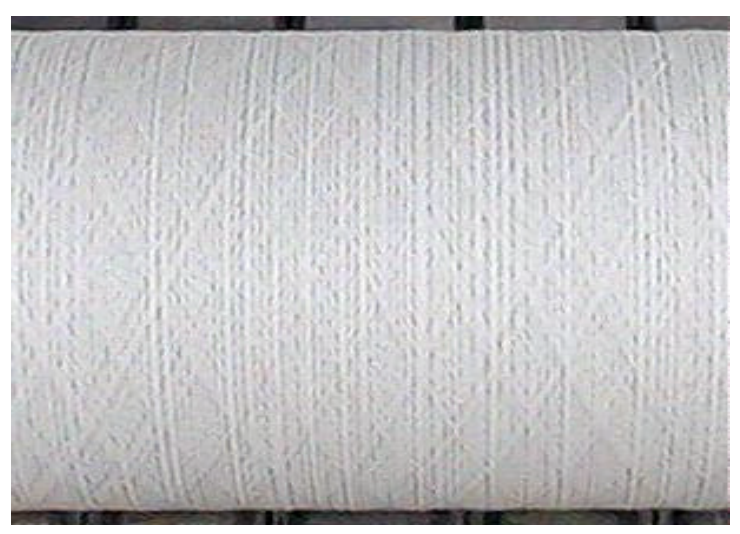

Figure 41 - Hoop winding with modified winder

Based on the equipment performance during the initial trials, several additional modifications were made including bearings and idler pulleys that were more durable, and a better support system for the tip of the mandrel. In addition, a longer traverse mechanism was required for the "hoop winder". After some challenges getting the new bearings and pulleys to fit properly, they functioned as required. The new pneumatic tip support system for the mandrel functioned well and allowed maximum rotational speed with minimal vibration, though it was somewhat awkward to operate during the installation and removal of the mandrel. The longer hoop-traverse mechanism allowed the membrane yarn to be wound along the entire length of the filter.

This winder was used to produce filters for the task of creating a membrane surface on the inside diameter of the PRD-66 filter (see Section 3.4.2). It was particularly essential for laying down a single "hoop layer" of yarn prior to winding the body of the filter.

It was noted that after winding less than six hot gas candle filters on the modified winder, a significant degradation in the winding pattern of the support yarn had occurred. The initially perfect diamond-pattern had many irregularities that could impact backpressure uniformity. The problem was caused by slack in the drive-chain that became gradually worse with continued use. By adding "chain guides" to the equipment, the smoothness with which the yarn guide moves from one end of the winder to the other was dramatically improved. If additional modifications are necessary, they will not be done under this program. 


\subsection{Field Testing of "Improved" PRD-66 Filter Elements}

\subsubsection{High Temperature High Pressure (HTHP) Testing at $\underline{W}$-STC}

Eight filter elements (four of each membrane type), manufactured during the first 10candle run of the "process capability demonstration", were submitted to Westinghouse Science and Technology Center. Upon arrival, all candles were measured for room temperature gas flow resistance, as shown in Figure 42 and Figure 43. Both sets of filter elements met the $\underline{\mathrm{W}}$-STC tolerance of $<1 \mathrm{in}-\mathrm{wg} / \mathrm{fpm}$ for as-manufactured candles.

During April 1997, one candle of each membrane type was subjected to a high temperature, high pressure (HTHP), simulated pressurized fluidized-bed combustion (PFBC) environment. Testing included exposure of the PRD-66 candles with alternate monolithic and advanced fiber reinforced candle filter elements in order to support pressurized circulating fluidized-bed combustion (PCFBC) test initiatives in Karhula, Finland. The filter array was subjected to 120 hours of steady state operating conditions at $843^{\circ} \mathrm{C}\left(1550^{\circ} \mathrm{F}\right)$, and subsequently 2,200 accelerated pulse cycles, and 12 mild thermal transient events.

Post-test inspection of the filter array indicated that both exposed PRD-66 filter elements remained intact. The following comments were noted:

- thin dust cake layer on both considered to be a "normal conditioned layer"

- no debonding or "divoting" of the outer membrane occurred

- no cracks were identified along the flange or body

- apparent heavier retention of fines in diamond pattern of PRD-66C versus PRD-66M

Post-test gas flow resistance measurements of the qualification-tested candles are provided in Figure 44. The coarse membrane (PRD-66C) element initially had a lower pressure drop in comparison to the medium membrane (PRD-66M) element; after qualification testing, this relationship was retained. These elements were subsequently subjected to mechanical strength characterization, x-ray diffraction, and microstructural analysis. 


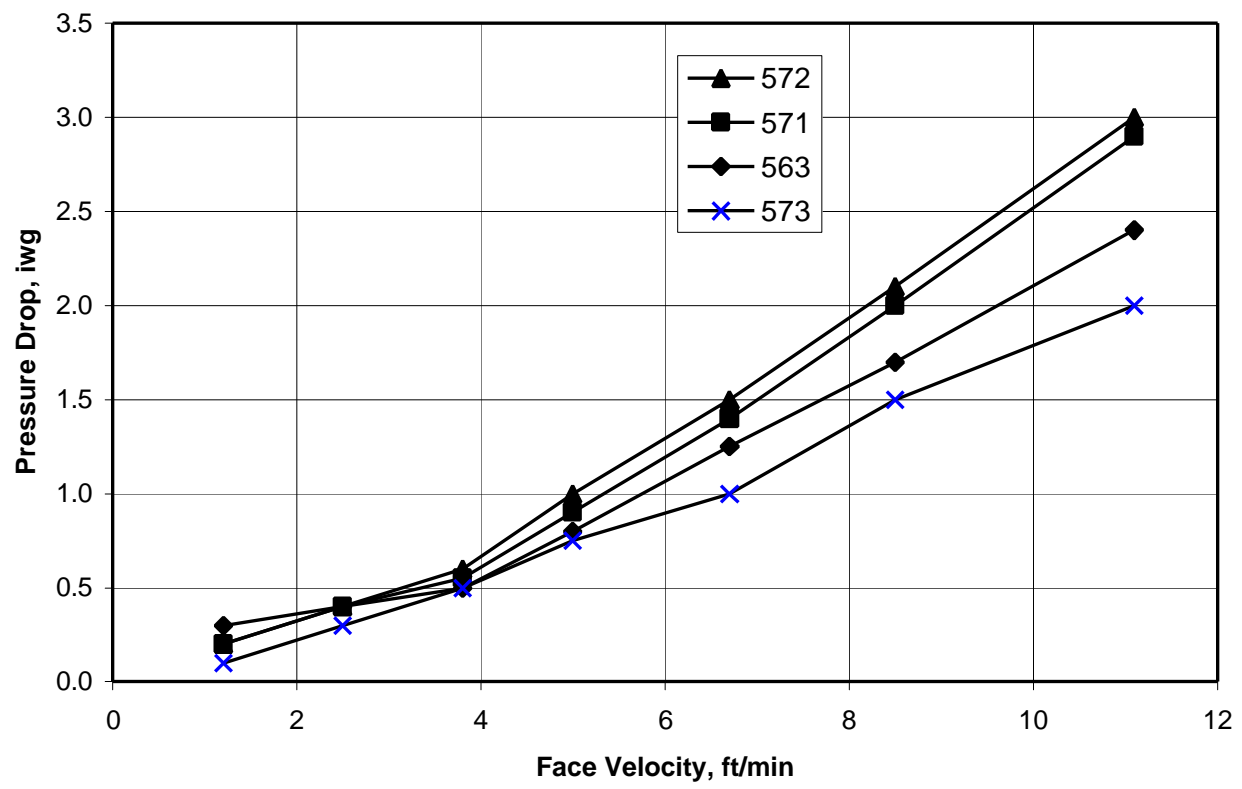

Figure 42 - PRD-66C - Room temperature gas flow resistance ${ }^{12}$

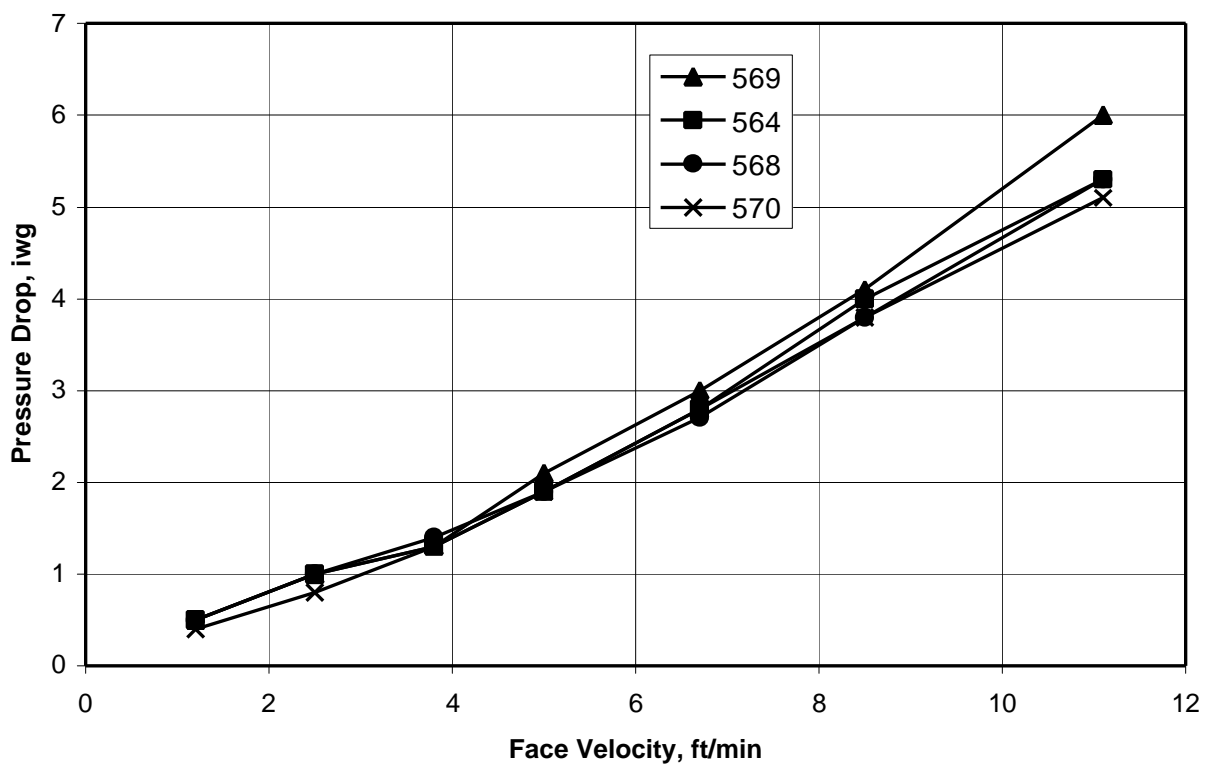

Figure 43 - PRD-66M - Room temperature gas flow resistance ${ }^{12}$ 


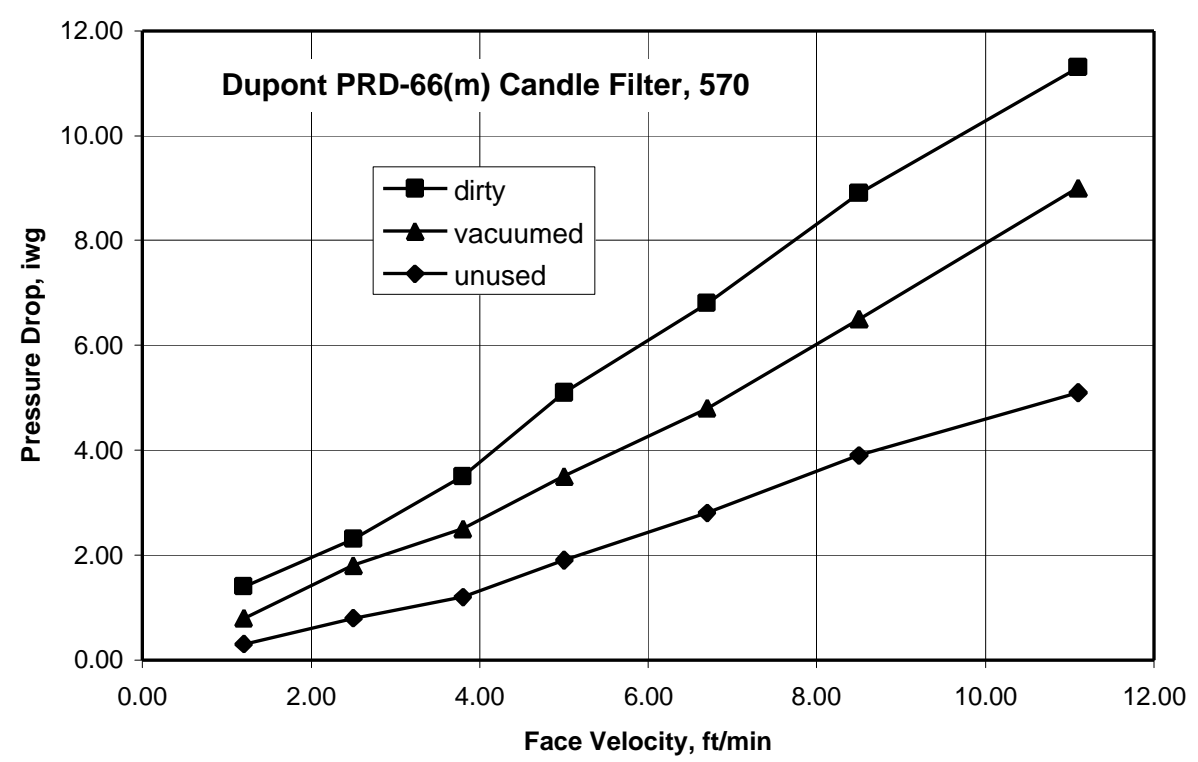

Figure 44 - Gas flow resistance of as-manufactured and HTHP-exposed PRD-66M elements ${ }^{12}$

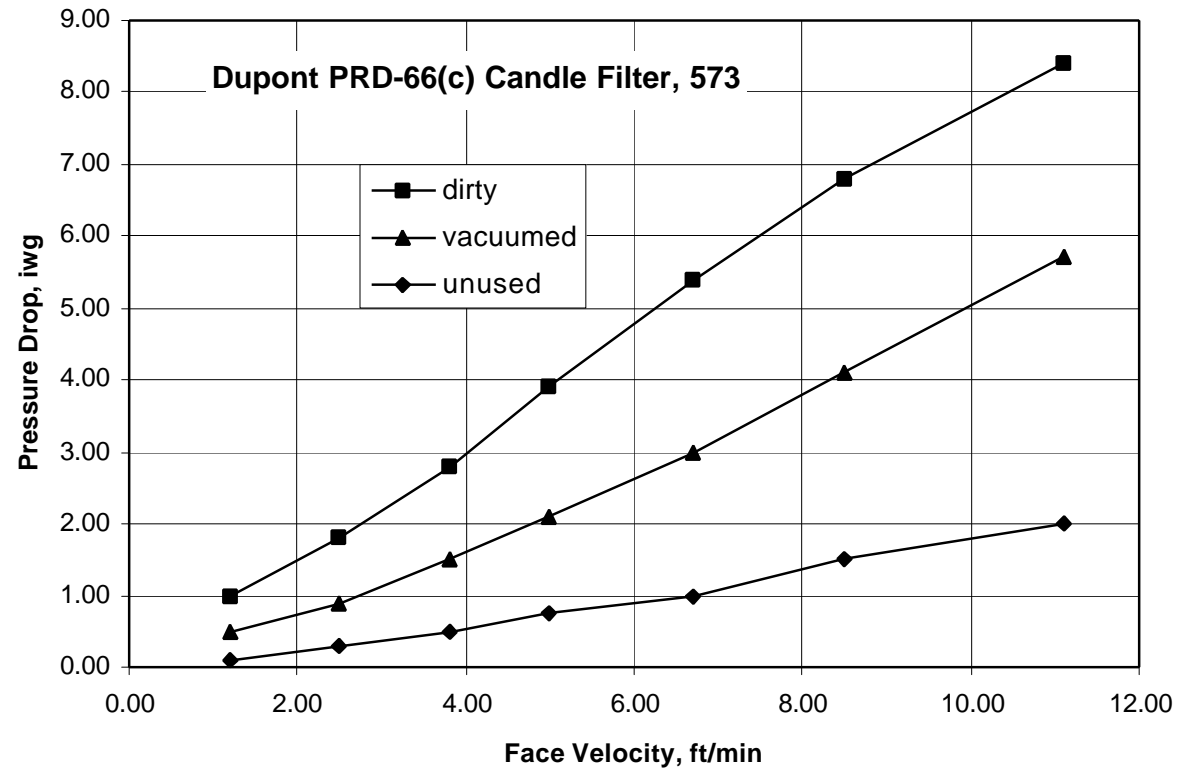

Figure 45 - Gas flow resistance of as-manufactured and HTHP-exposed PRD-66C elements ${ }^{12}$ 
W-STC characterized the mechanical properties the two tested elements, along with one as-manufactured candle of each membrane type. ${ }^{12}$ Table 11 summarizes the compressive and tensile c-ring tests that were conducted; the data suggests that the strength of the coarse and medium membrane "exposed" elements tended to be greater than the strength of comparable asmanufactured elements. M. A. Alvin of $\underline{\mathrm{W}}-\mathrm{STC}$ feels that this conclusion is supported by similar results obtained during other simulated and field exposures. ${ }^{4}$ It had been postulated that an increase in strength could result from the bulk versus barrier filtration characteristics of the material, whereby submicron and micron fines penetrate through the membrane of the PRD-66 filter element and become trapped within the filter wall. Under these conditions, trapped ash could cause significant problems during field operation, particularly if thermal expansion occurs within the filter wall during plant startup cycles, ${ }^{5}$ or hydration of the ash resulted during thermal shutdown cycles (Section 3.3.3). In relation to alternate filter elements, ${ }^{6}$ the PRD-66 candle filters were considered to be "moderately low" load-bearing (Table 12). Additional material properties as burst strength, modulus, and Poisson's ratio, which were developed at Westinghouse, are provided in Table 13.

\begin{tabular}{|c|c|c|c|c|c|}
\hline \multicolumn{6}{|c|}{$\begin{array}{c}\text { ROOM TEMPERATURE AND PROCESS STRENGTH OF THE } \\
\text { AS-MANUFACTURED AND QUALIFICATION-TESTED } \\
\text { DUPONT PRD-66 CANDLE FILTERS } \\
\end{array}$} \\
\hline $\begin{array}{c}\text { Candle } \\
\text { Identification }\end{array}$ & \multirow[t]{2}{*}{ Status } & \multicolumn{2}{|c|}{$\begin{array}{c}\text { C-Ring Compressive Strength, } \\
\text { psi }\end{array}$} & \multicolumn{2}{|c|}{$\begin{array}{l}\text { C-Ring Tensile Strength, } \\
\text { psi }\end{array}$} \\
\hline Number & & $25-\operatorname{deg} C$ & 843-degC & $25-\operatorname{deg} C$ & $843-\operatorname{deg} C$ \\
\hline \multicolumn{6}{|c|}{ DuPont PRD-66 (Coarse Membrane) } \\
\hline$D-563 c$ & As-Manufactured & $955+/-62(9)$ & $962+/-92(8)$ & $809+/-154(9)$ & $1009+/-103(7)$ \\
\hline D-573c & Qualification Tested & $1214+/-67(9)$ & $1210+/-86(9)$ & $990+/-82(9)$ & $1195+/-166(9)$ \\
\hline \multicolumn{6}{|c|}{ edium Membrane) } \\
\hline D-564m & As-Manufactured & $990+/-130(9)$ & $883+/-79(9)$ & $846+/-105(9)$ & 918+/-104 (9) \\
\hline D-570m & Qualification Tested & $1021+/-127(9)$ & $1019+/-88(9)$ & $973+/-165(9)$ & $1193+/-149(8)$ \\
\hline
\end{tabular}

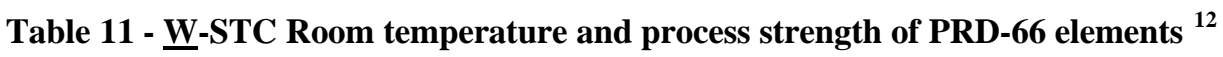




\begin{tabular}{|c|c|c|c|c|c|}
\hline \multicolumn{6}{|c|}{$\begin{array}{l}\text { ULTIMATE LOAD APPLIED DURING STRENGTH CHARACTERIZATION } \\
\text { OF THE AS-MANUFACTURED AND QUALIFICATION-TESTED } \\
\text { DUPONT PRD-66 CANDLE FILTERS }\end{array}$} \\
\hline $\begin{array}{c}\text { Candle } \\
\text { Identification }\end{array}$ & \multirow[t]{2}{*}{ Status } & \multicolumn{2}{|c|}{$\begin{array}{l}\text { C-Ring Compressive } \\
\text { Load-to-Failure, psi }\end{array}$} & \multicolumn{2}{|c|}{$\begin{array}{c}\text { C-Ring Tensile } \\
\text { Load-to-Failure, psi }\end{array}$} \\
\hline Number & & $25-\operatorname{degC}$ & $843-\operatorname{deg} C$ & $25-\operatorname{deg} C$ & $843-\operatorname{deg} C$ \\
\hline \multicolumn{6}{|c|}{ DuPont PRD-66 (Coarse Membrane) } \\
\hline $\mathrm{D}-563 \mathrm{c}$ & As-Manufactured & $8.2+/-0.5(9)$ & $8.2+/-0.9(8)$ & $5.2+/-1.1(9)$ & $6.7+/-0.7(7)$ \\
\hline D-573c & Qualification Tested & $10.3+/-0.6(9)$ & $10.3+/-0.6(9)$ & $6.4+/-1.2(9)$ & $7.6+/-1.0(9)$ \\
\hline \multicolumn{6}{|c|}{ DuPont PRD-66 (Medium Membrane) } \\
\hline D-564m & As-Manufactured & $8.0+/-0.9(9)$ & $7.3+/-0.6(9)$ & $5.2+/-0.6(9)$ & $5.7+/-0.6(9)$ \\
\hline D-570m & Qualification Tested & $8.3+/-1.0(9)$ & $8.3+/-0.8(9)$ & $6.1+/-0.9(9)$ & $7.4+/-0.8(8)$ \\
\hline
\end{tabular}

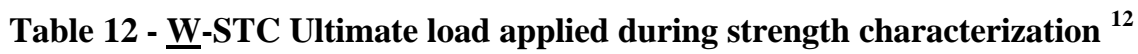

\begin{tabular}{|c|c|c|c|c|c|}
\hline \multicolumn{6}{|c|}{$\begin{array}{c}\text { MATERIAL PROPERTIES } \\
\text { OF THE AS-MANUFACTURED AND QUALIFICATION-TESTED } \\
\text { DUPONT PRD-66 CANDLE FILTERS } \\
\end{array}$} \\
\hline $\begin{array}{c}\text { Candle } \\
\text { Identification } \\
\text { Number }\end{array}$ & Status & $\begin{array}{c}\text { Burst } \\
\text { Pressure, } \\
\text { psi }\end{array}$ & $\begin{array}{l}\text { Ultimate } \\
\text { Hoop } \\
\text { Stress, psi }\end{array}$ & $\begin{array}{r}\text { Modulus, } \\
\text { psi x } 10^{6}\end{array}$ & $\begin{array}{c}\text { Poisson's } \\
\text { Ratio }\end{array}$ \\
\hline \multicolumn{6}{|c|}{ DuPont PRD-66 (Coarse Membrane) } \\
\hline D-563c & As-Manufactured & 148 & 555 & 7.96 & 0.86 \\
\hline D-573c & Qualification Tested & 158 & 597 & 6.11 & 0.82 \\
\hline \multicolumn{6}{|c|}{ DuPont PRD-66 (Medium Membrane) } \\
\hline$\overline{D-564 m}$ & As-Manufactured & 180 & 691 & 7.09 & 0.84 \\
\hline D-570m & Qualification Tested & 170 & 653 & 5.42 & 0.84 \\
\hline
\end{tabular}

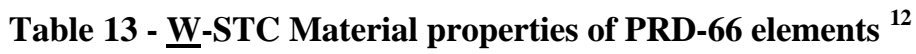

Additional strength testing was conducted by DuPont Lanxide Composites on segments of the same "exposed" filter elements tested by W-STC and on two different as-manufactured candles. These results, shown in Table 14, DO NOT support the Westinghouse conclusions. The "exposed" PRD-66C had a higher strength, however the "exposed" PRD-66M had a lower strength. The data suggests that the candle-to-candle strength variability of the material outweighs any effect of exposure. It was interesting to note, however, that the $\underline{\mathrm{W}}$-STC c-ring strength values and the DLC o-ring strength values for candles \#570 and \#573 were very similar.

\begin{tabular}{|l|c|c|c|}
\hline Candle ID\# & Status & O-Ring Comp.Str & Load-to-Failure \\
\hline PRD-66C & & & \\
\hline 566C & As-Manufactured & $1087 \pm 80(11)$ & $41.5 \pm 3.1(11)$ \\
\hline 573C & Qualification Tested & $1252 \pm 44(5)$ & $45.6 \pm 3.6(5)$ \\
\hline PRD-66M & & & \\
\hline $567 \mathrm{M}$ & As-Manufactured & $1229 \pm 117(11)$ & $44.7 \pm 3.9(11)$ \\
\hline $570 \mathrm{M}$ & Qualification Tested & $1095 \pm 184(5)$ & $37.2 \pm 6.7(5)$ \\
\hline
\end{tabular}

Table 14 - DLC Diametrical compression testing of HTHP-exposed \& unexposed candles 


\subsubsection{PCFBC Exposure at Karhula}

A 581-hour exposure of PRD-66C filter elements was conducted in Foster Wheeler's pressurized circulating fluidized-bed combustion (PCFBC) test facility in Karhula, Finland. Analysis of an exposed filter was conducted under Task 3.2.

Seven candles began the test in early September. Table 15 (provided by Westinghouse) identifies the operating conditions experienced by the PRD-66C Hot Gas Candle Filters in Westinghouse's Advanced Particulate Filter cluster during the TS2-1997 test campaign

\begin{tabular}{|c|c|}
\hline \multicolumn{2}{|c|}{$\begin{array}{l}\text { Pressurized Circulating Fluidized-bed Combustion Testing at the } \\
\text { Foster Wheeler Test Facility in Karhula, Finland - TS2-97 }\end{array}$} \\
\hline Date & September 4, 1997 - November 7, 1997 \\
\hline Number of Filter Elements Tested & 8 \\
\hline Filter Operating Temperature, deg.C & $700-750$ \\
\hline Filter Operating Pressure, bar & $9.5-11$ \\
\hline Coal Feed & Eastern Kentucky \\
\hline Sorbent & Florida Limestone \\
\hline Time, hrs & $581(6)^{*}, 342(1), 239(1)$ \\
\hline Face Velocity, cm/sec & $2.8-4.0$ \\
\hline Particle Load, ppmw & $6000-9000$ \\
\hline Particle Size, microns & $<1-150$ \\
\hline Thermal Excursions & None \\
\hline Number of Startup/Shutdown Cycles & 7 \\
\hline
\end{tabular}

Table 15 - Karhula PCFBC test conditions

After 239 hours, the system was turned off and all elements were examined. Significant quantities of ash were found on the "clean side" of the system. All candles were removed and cleaned by vacuuming and washing. One PRD-66C candle broke at the flange when it was removed; some force had been necessary to dislodge the flange from the holder assembly. When the run was restarted, a new PRD-66C candle was put in its place. The test concluded 342 hours later. 
At the conclusion of the run, the six PRD-66C elements that were exposed for the entire 581 hours, and the one candle that was exposed for a total of 342 hours, all looked good. All but one of the elements had been cleaned by brushing and vacuuming prior to inspection, see photograph in Figure 46. There was no sign of any material deterioration in the possible forms of "divots", abrasion, poor membrane adhesion, or cracking. A significant amount of ash, however, was observed in the wall of the inside diameter, though it was much less for the element that was only exposed for 342 hours.

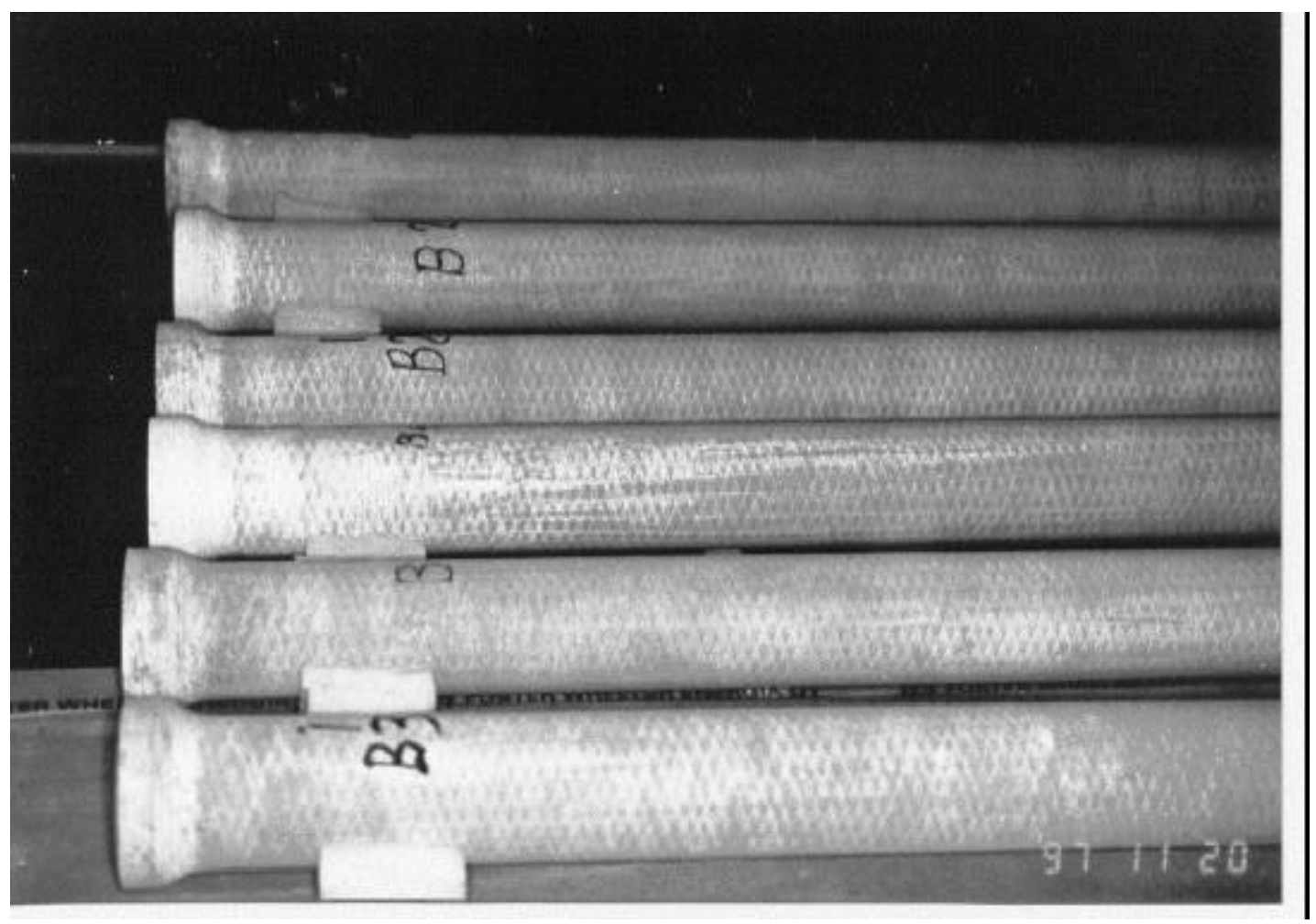

Figure 46 - Karhula-exposed PRD-66C filters

A single candle was examined before any ash had been cleaned from the material. A conditioned ash cake layer, approximately $2 \mathrm{~mm}$ thick, had formed along the outside diameter, see photograph in Figure 47. The ash was soft and easily removable by handling or by brushing. The inside diameter was also caked with ash, approximately $2 \mathrm{~mm}$ thick, with at least six inches of loose ash present in the tip of the candle. 


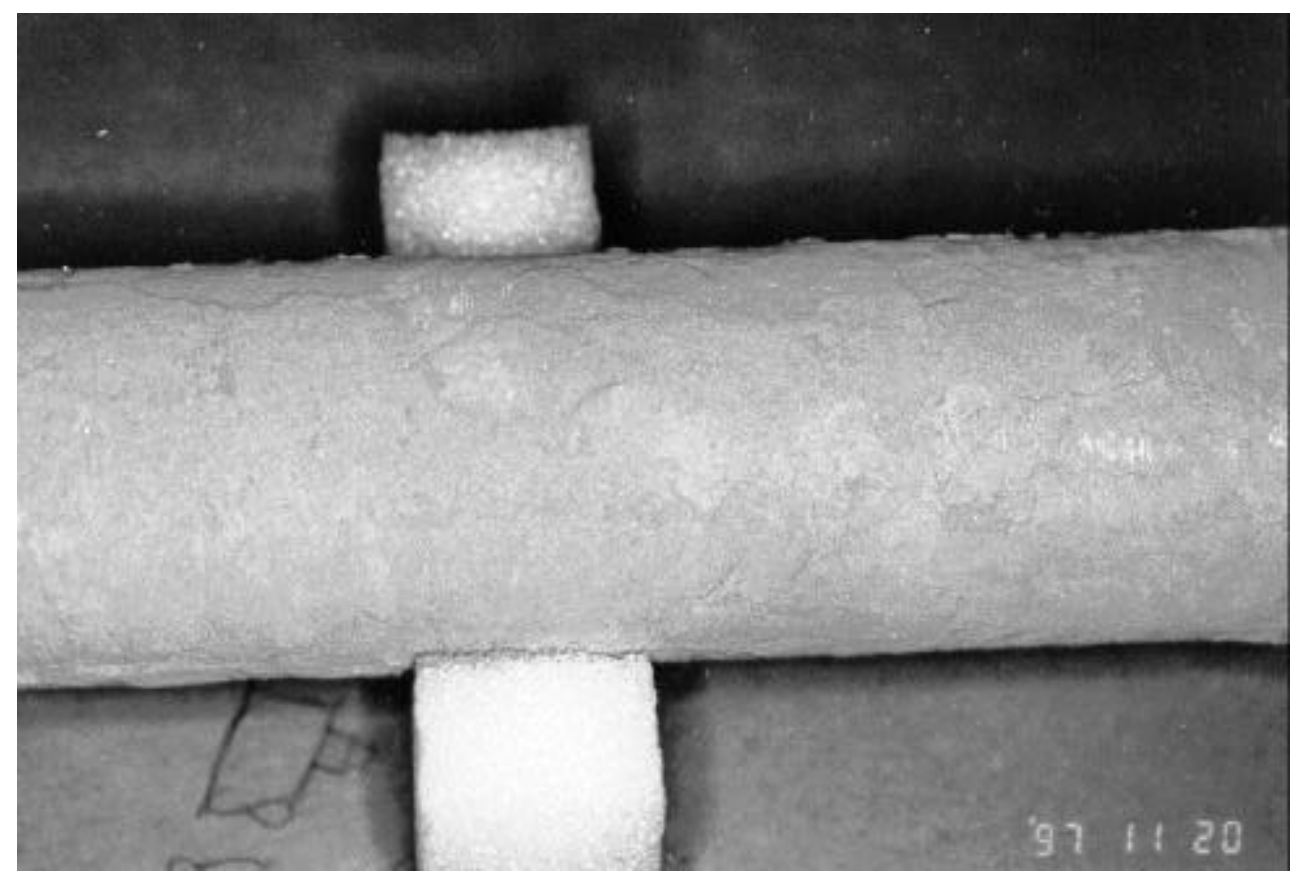

Figure 47 - Outside diameter of Karhula-exposed element before ash removal

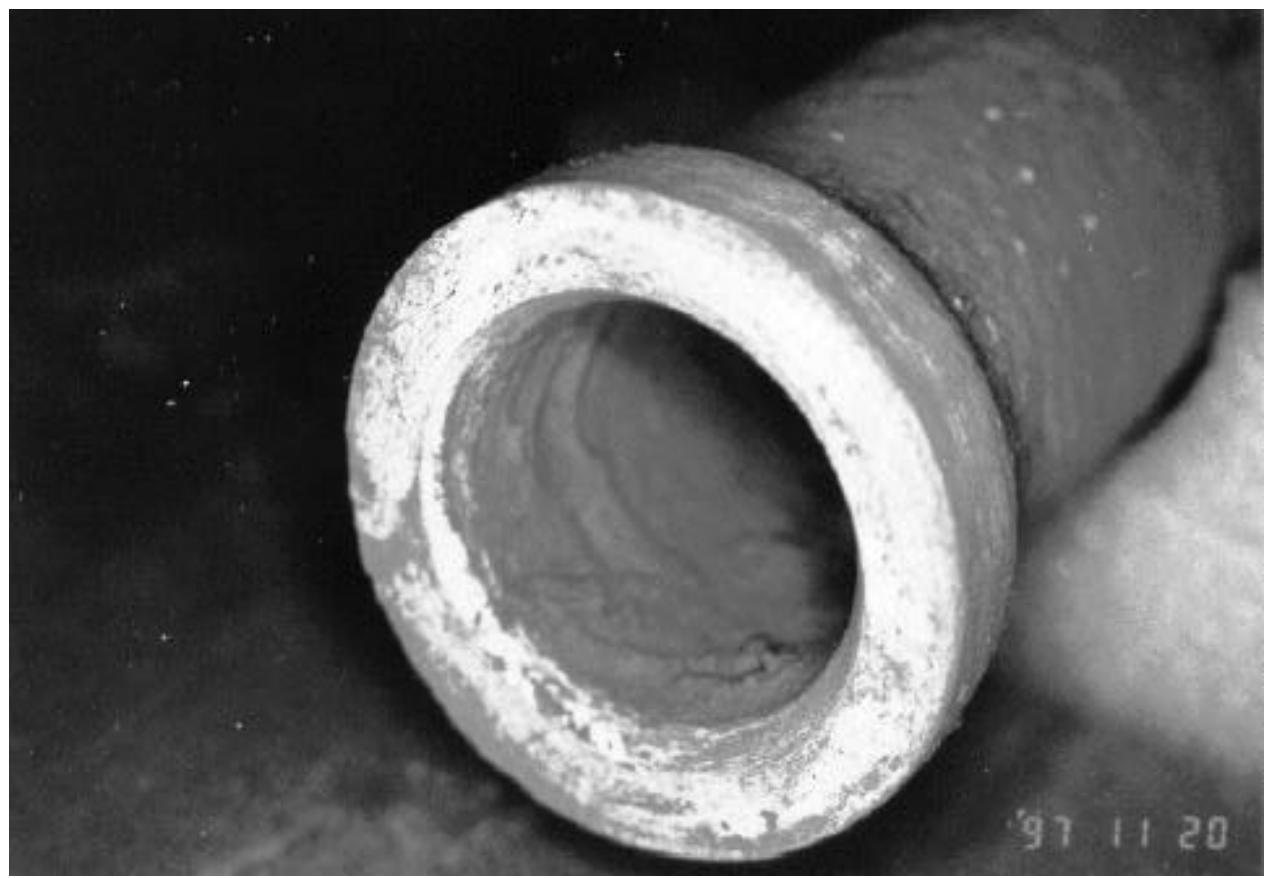

Figure 48 - Inside diameter of Karhula-exposed element before ash removal

All candles were vacuum-cleaned, inside and out, prior to inspection, after which, differential pressure measurements were conducted by Foster Wheeler personnel, see Figure 49. In summary, all elements showed significantly higher backpressure, with the exception of the single candle that was installed after the "239-hour shutdown", which had a slight increase in 
backpressure. FW has attributed the plugging of the other filters to the presence of significant quantities of ash on the "clean side", rather than the length of exposure.

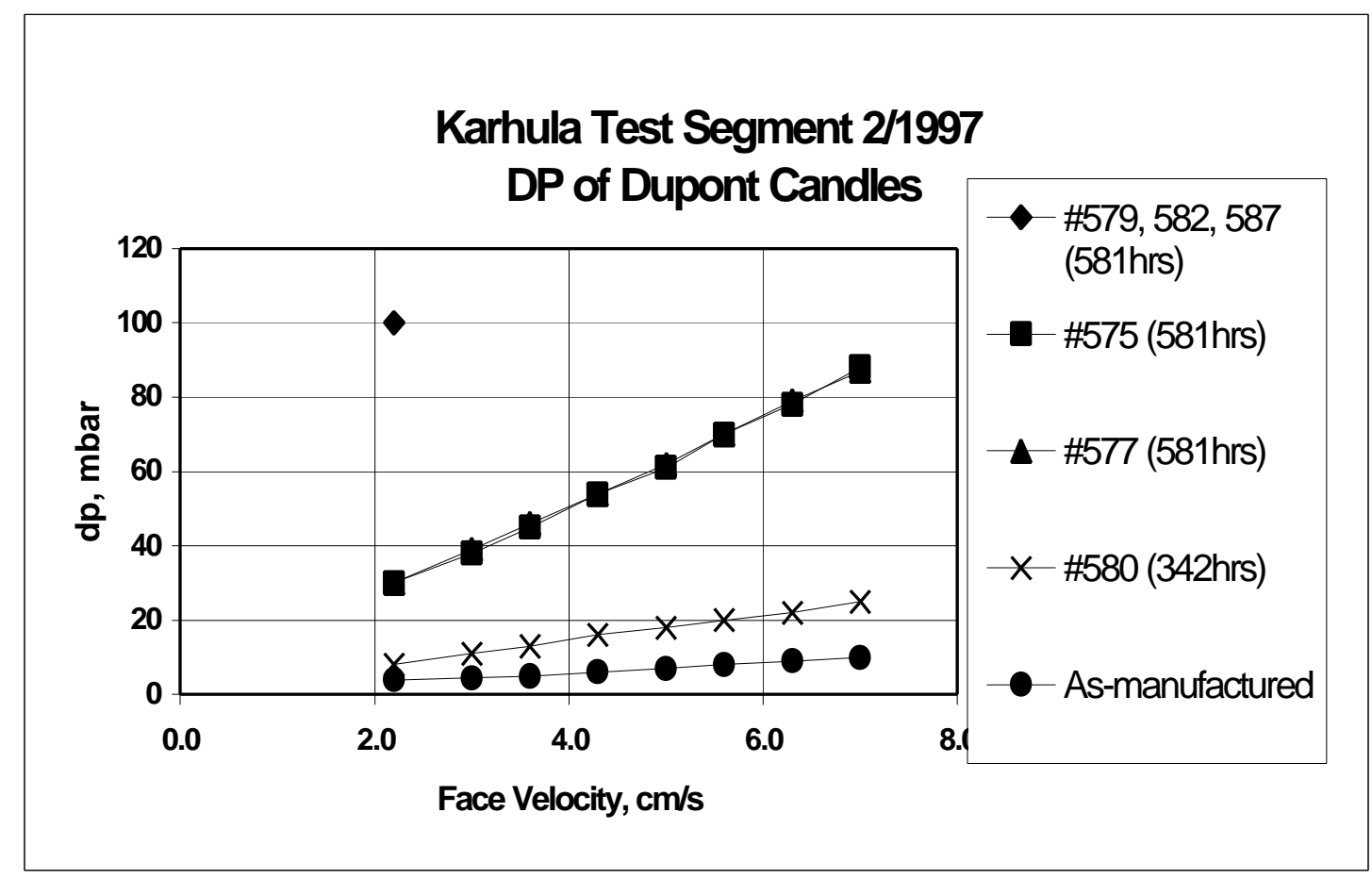

Figure 49 - Differential pressure of Karhula filters measured by Foster Wheeler

One of the candles with the full exposure time (\#577), and the candle, which broke during removal after 239 hours (\#591), were shipped to DLC for analysis. Unfortunately, both broke into at least three pieces during transport.

\subsubsection{Visual Inspection for Ash Penetration in Karhula-Exposed Element}

Samples of candle \#577 (with 581 exposure hours) were prepared by "fast-fracture", to expose a cross-section of the wall. The contrast between the dark (orange-brown) ash and the white PRD-66 support material made it easy to determine where obvious ash penetration had occurred. Figure 50 is a photograph of a particular sample in which the support yarn was exposed at two distinct levels: just below the membrane and approximately $4 \mathrm{~mm}$ below the membrane (mid-way through the wall). The presence of ash mid-way through the wall was no surprise, since a process upset had occurred during the Karhula exposure, which introduced large quantities of ash into the ID of the filter elements. The most significant observation was that there was no ash within $1-2 \mathrm{~mm}$ of the membrane. Figure 51 is an enlargement of that area shown in Figure 50. The ash is clearly seen trapped in the membrane, while the yarns of the support 
structure immediately below are clean and white. This indicates that the new PRD-66C membrane (with nominal 25-micron pores) is an effective surface filter for PCFBC applications. It is significant that, no "divots" occurred despite the large volumes of ash that penetrated from the "clean side".

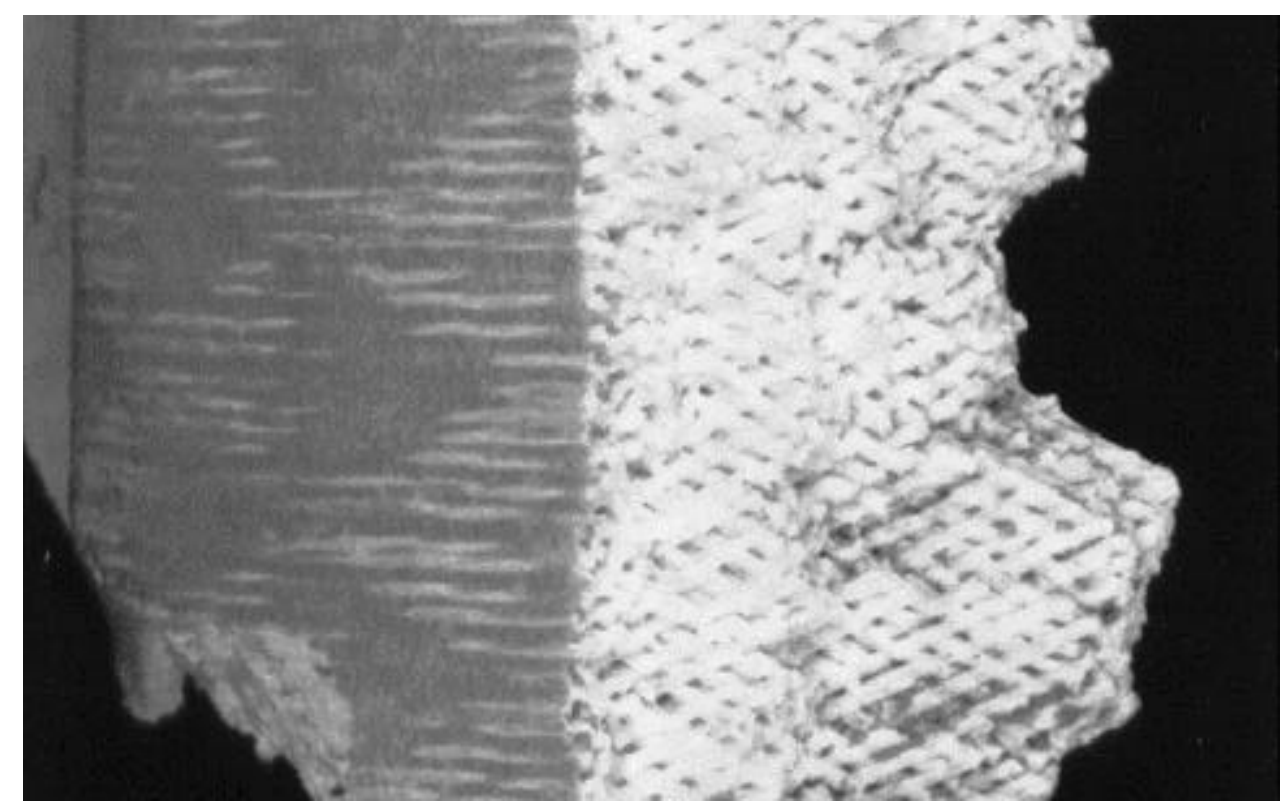

Figure 50 - Wall interior of Karhula-exposed candle \#577

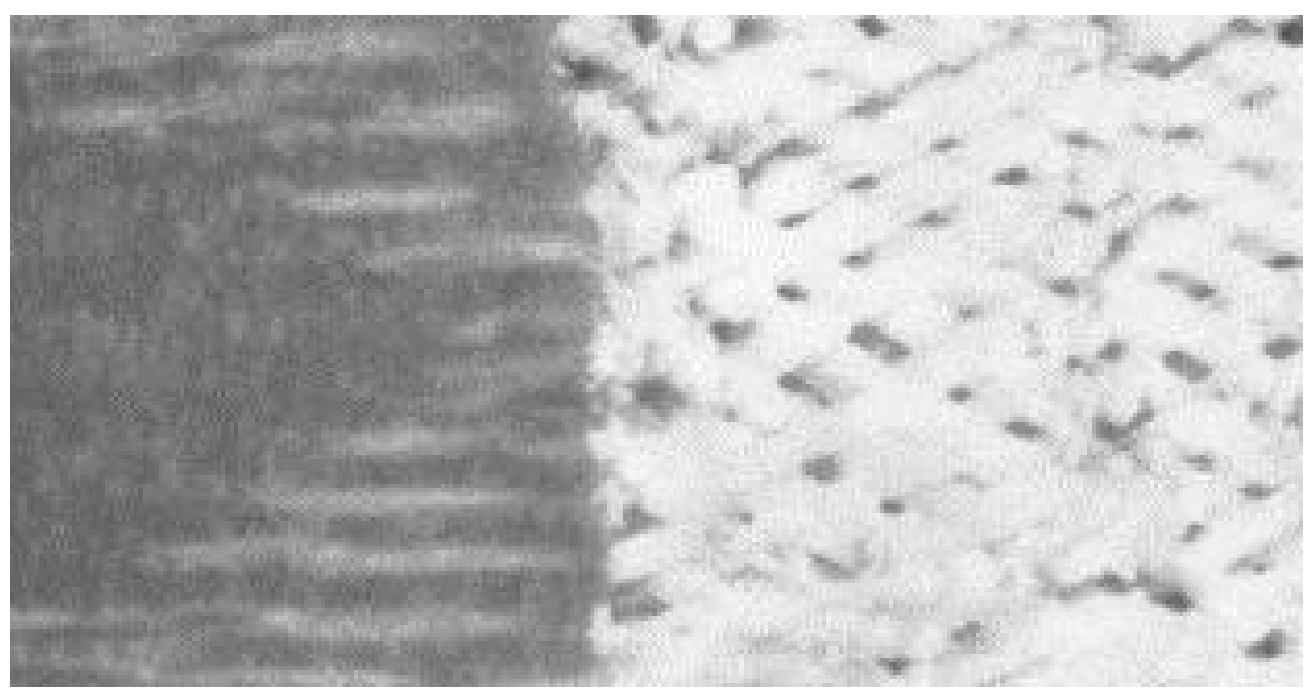

Figure 51 - Close-up of \#577 - OD surface and 1-2mm below

FW also shipped approximately one liter of PCFBC ash that could be used to conduct a particle infiltration test (PIT) on a "sister" candle filter. The test was performed on a two-inch segment of unused candle \#576. The results confirmed the observations made on the Karhulaexposed candle; no penetration of ash through the membrane was detected. 


\subsubsection{Microstructural Analysis of Karhula-Exposed and Unexposed Elements}

A series of scanning electron microscopy (SEM) photographs were taken of different features of the exposed candle \#577 and the unexposed candle \#576. In the following photos, comparisons were made of the exposed outside diameter surfaces. In Figure 52, the structure of the unexposed membrane has coarse alumina grains speckled with fine grains of the fusible binder, when viewed at 300X. By comparison, the exposed candle in Figure 53 and Figure 54 show similar irregularities which have been "smoothed-over" by the presence of ash.

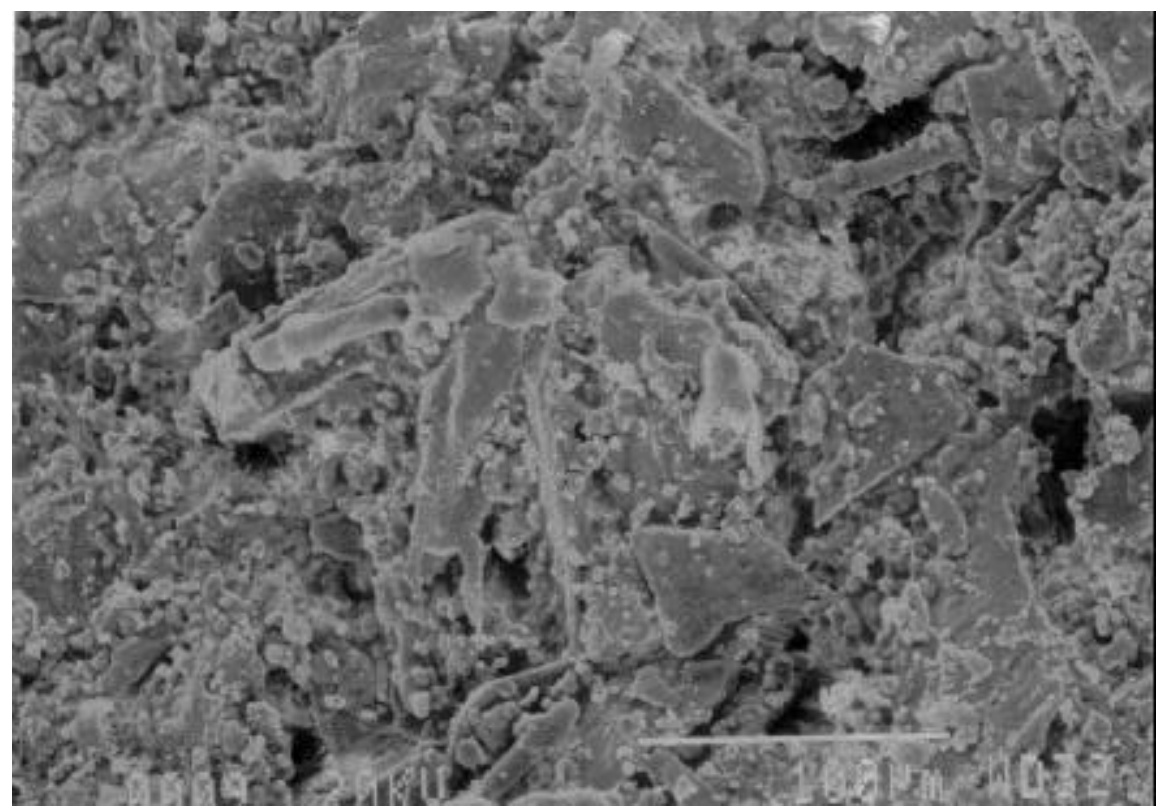

Figure 52 - 300X - UNEXPOSED candle surface

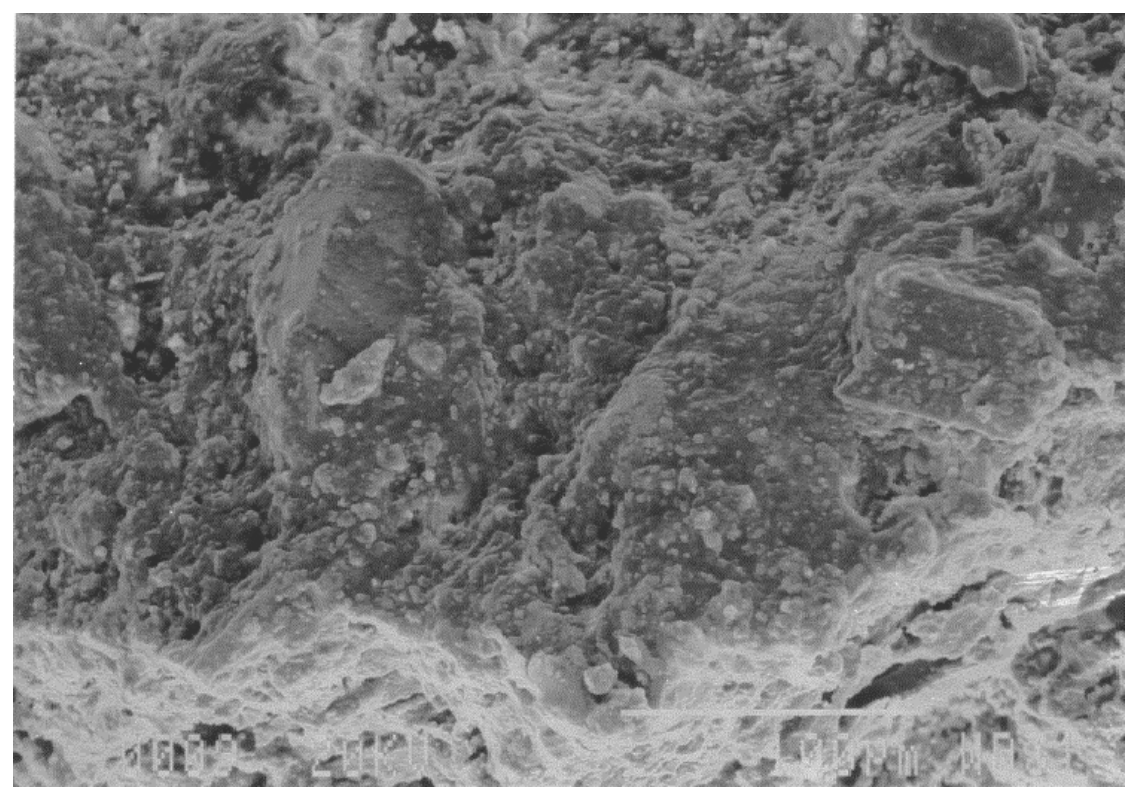

Figure 53 - 300X - EXPOSED candle surface 


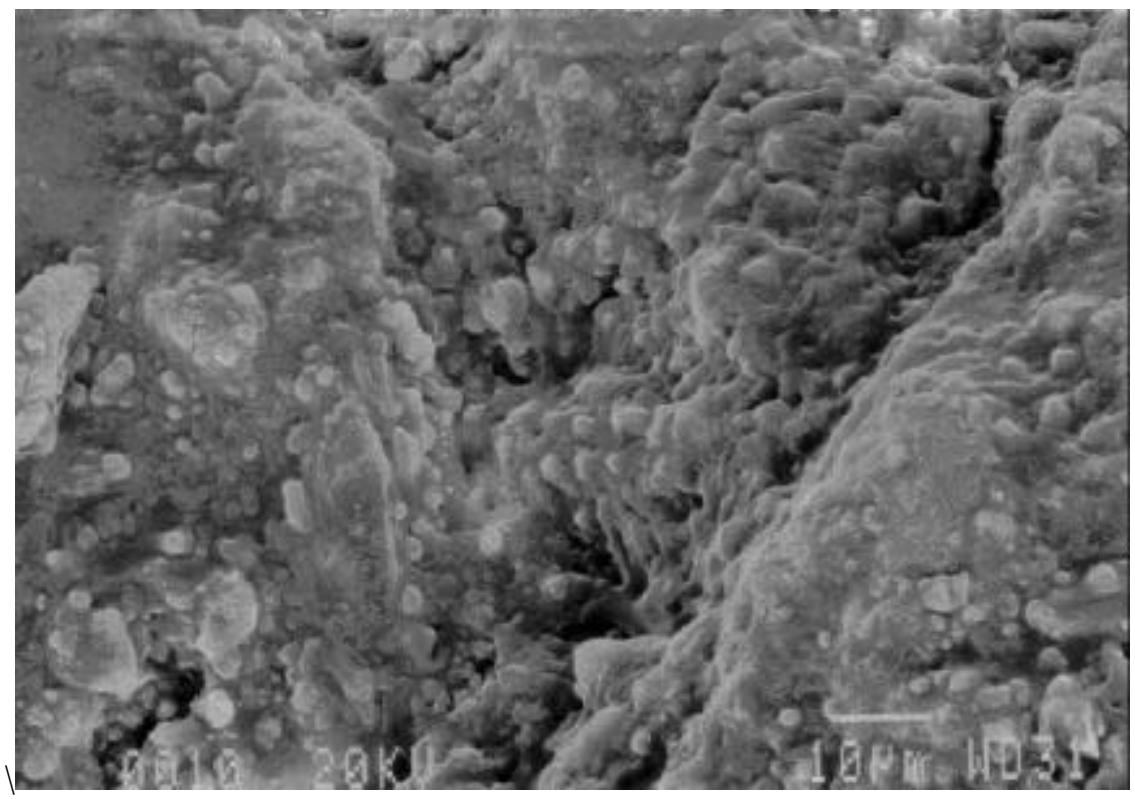

Figure 54 - 1,000X - EXPOSED candle surface

In the following photos, cross-sections of the particulate membrane filler were exposed by fast-fracture and evidence of any ash deposits were sought. By making comparisons with an unexposed filter (Figure 55), no obvious trace of ash could be discerned in Figure 56; no significant difference in the sharp edges of the alumina particles of the membrane were observed.

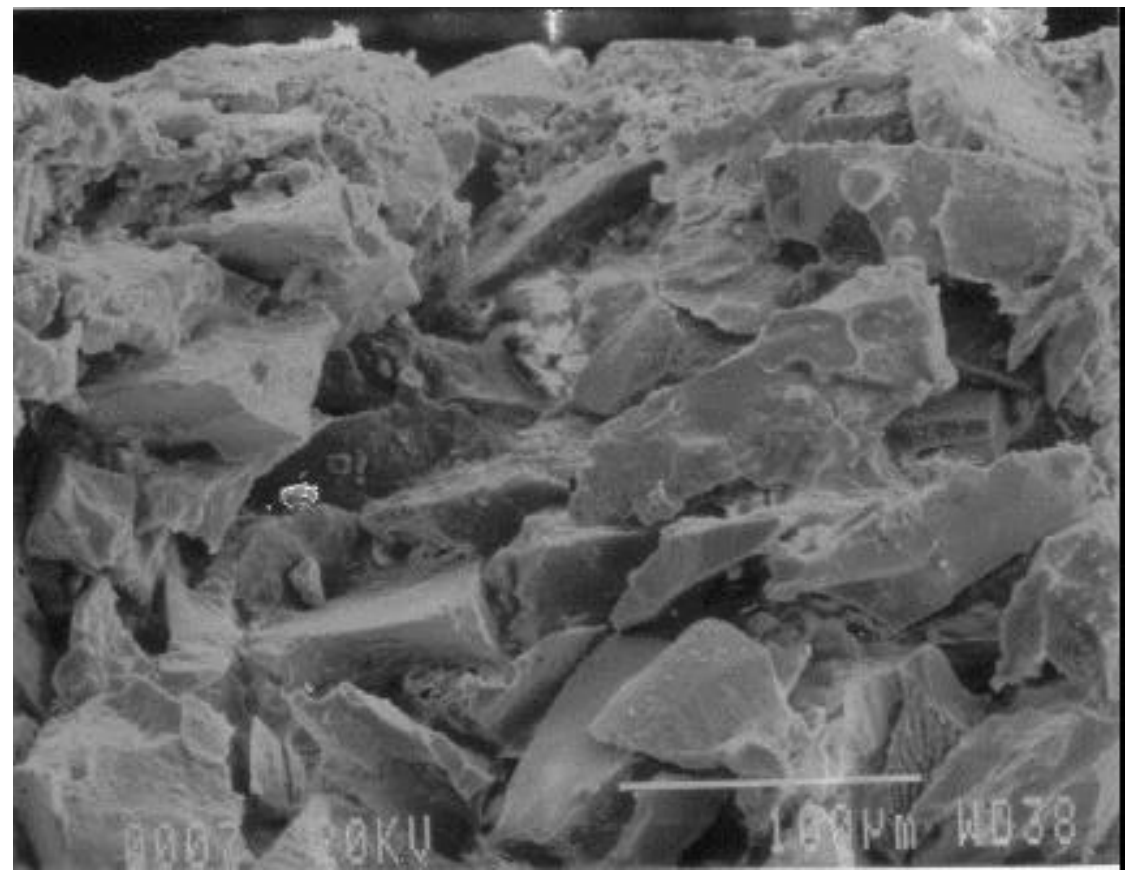

Figure 55 - UNEXPOSED CANDLE, cross-section of membrane filler (300X) 


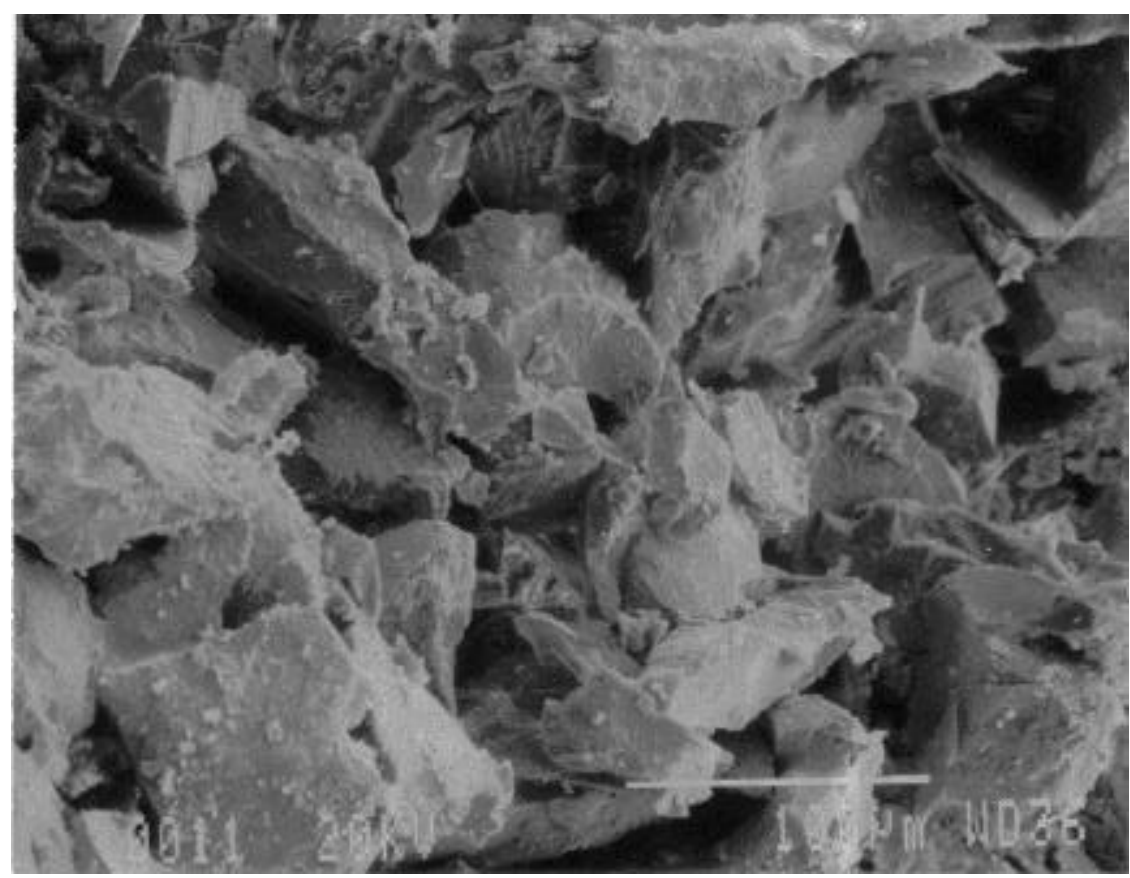

Figure 56 - EXPOSED CANDLE, cross-section of membrane filler (300X)

In the following photos, the SEM was focused on the region of the support wall within $3 \mathrm{~mm}$ of the OD surface. The exposed candle in Figure 58 showed no obvious evidence of ash entrainment when compared to the unexposed candle in Figure 57.

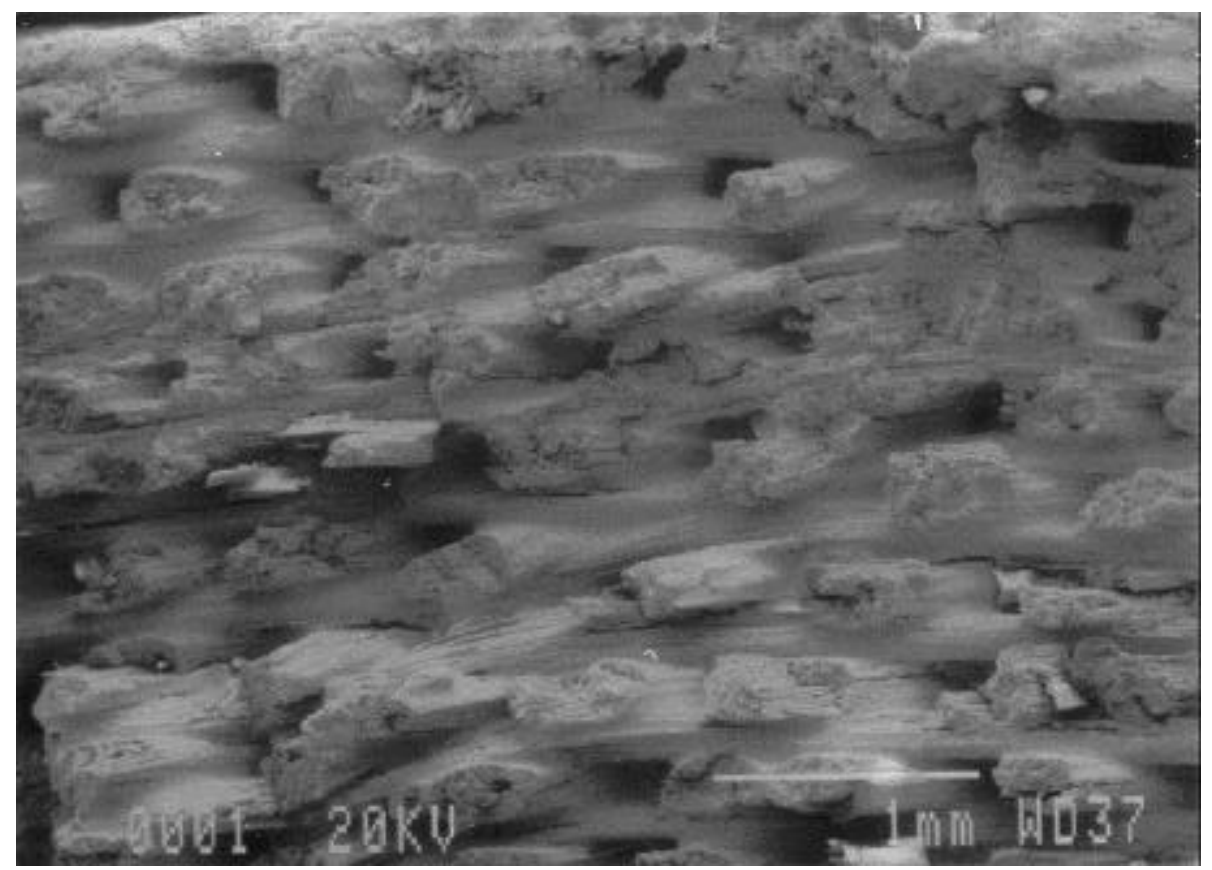

Figure 57 - 25X, fast-fracture - UNEXPOSED CANDLE, interior of support wall 


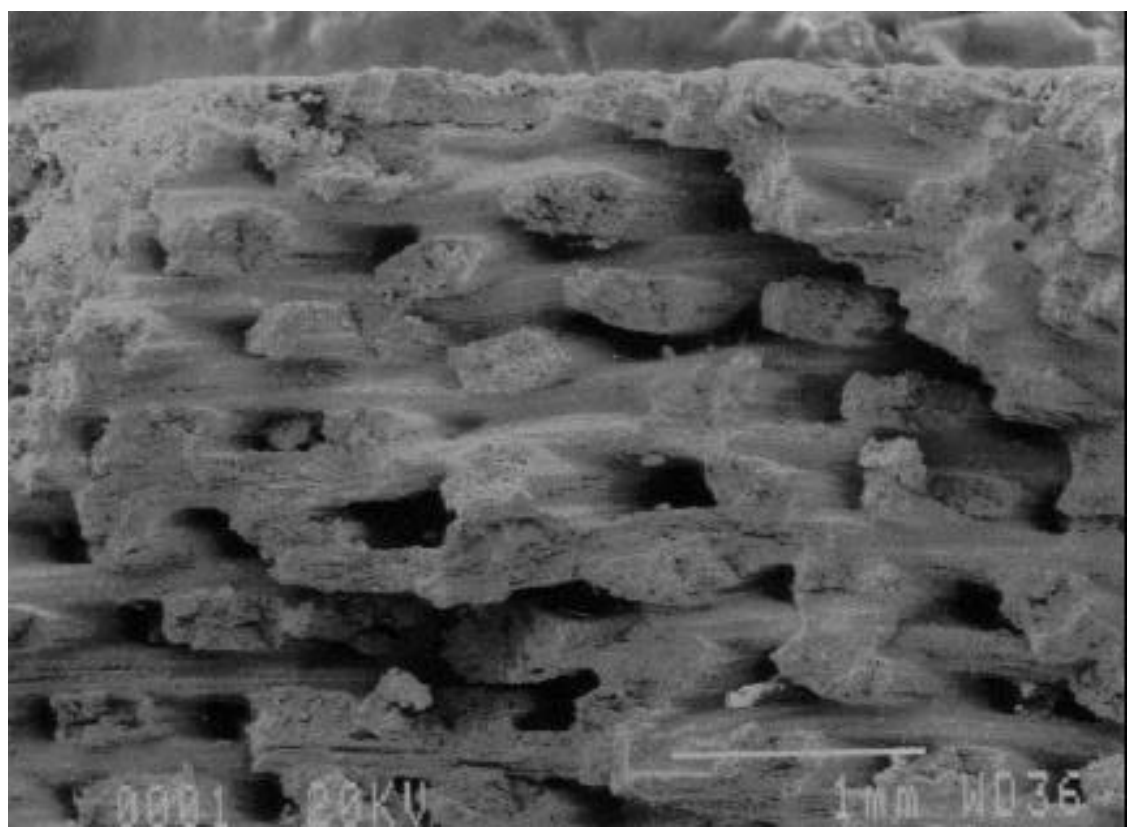

Figure 58 - 25X, fresh-fracture - EXPOSED CANDLE, interior of support wall

Upon closer examination of the $1 \mathrm{~mm}$ area directly below the membrane, the natural microcracks in the unexposed material are visible along the surface of the filament structures (Figure 59). These microcracks were also visible in the Figure 60 photo of the exposed candle; if ash penetration had occurred, a smoothening or filling of those features may have resulted. These micrographs support the observation that no detectable penetration of ash through the membrane layer occurred.

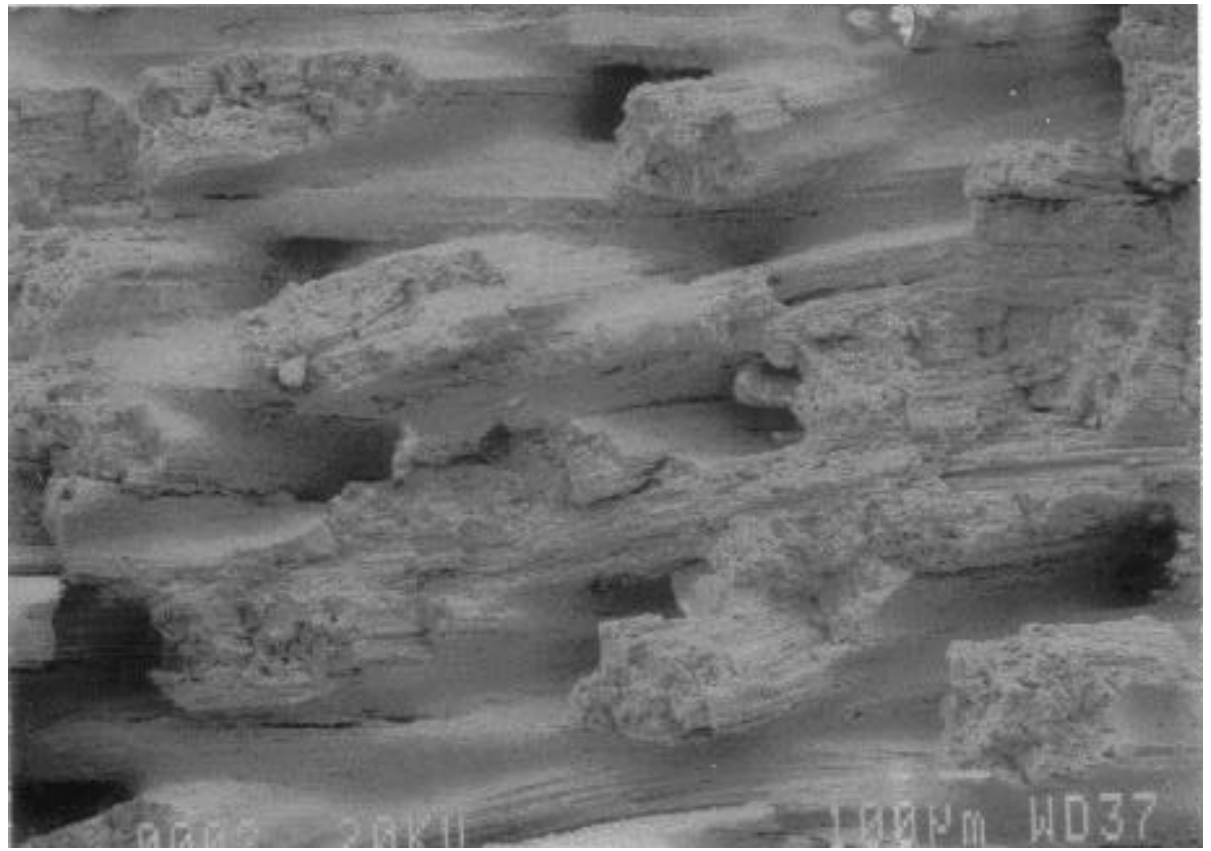

Figure 59 - 50X, fast-fracture - UNEXPOSED CANDLE, interior of support wall 


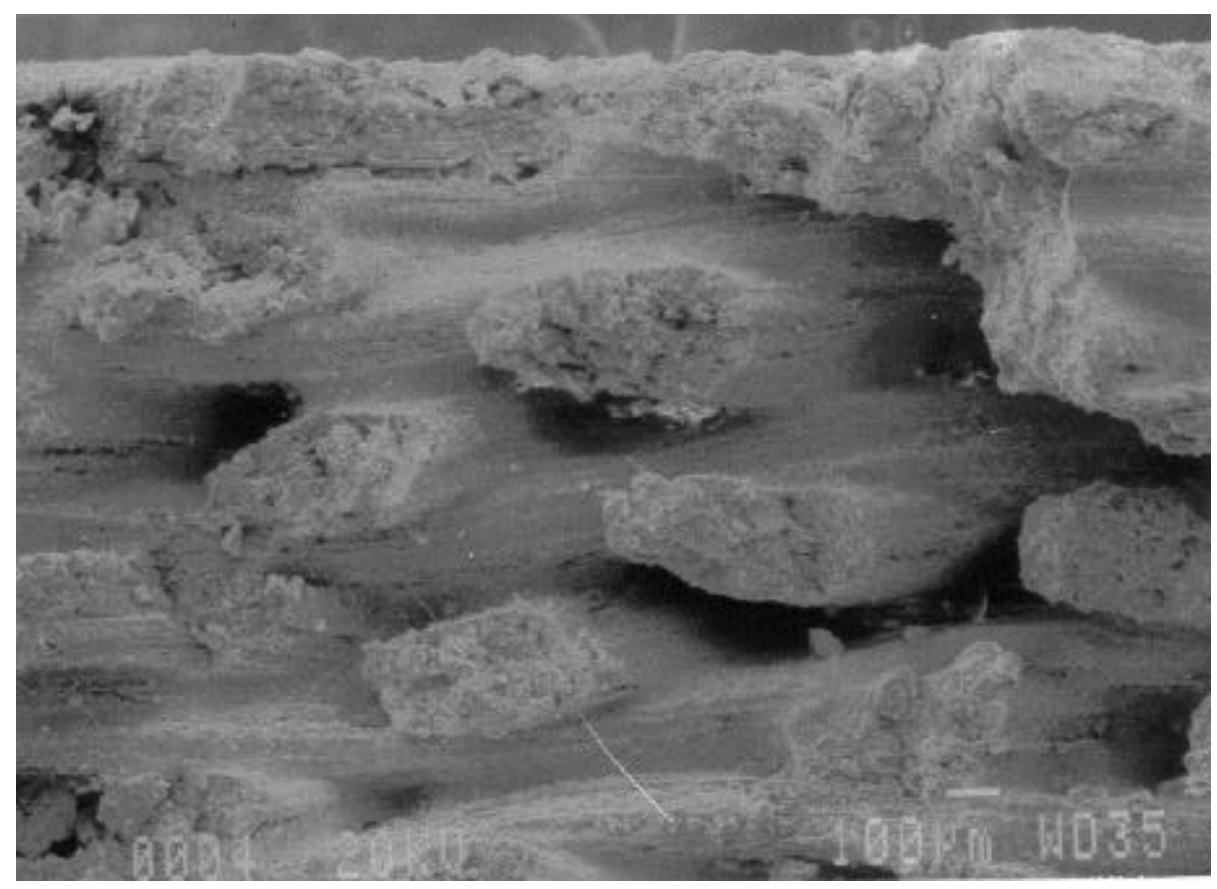

Figure 60 - 50X, fast-fracture - EXPOSED CANDLE, interior of wall support

In Figure 61 through Figure 64, the conditions of the filament structures were examined for evidence of any change resulting from the exposure environment. Figure 61 and Figure 62 each show the cross-section of a single "yarn bundle" at 300X magnification. Each yarn bundle originally consisted of hundreds of filaments. During the firing process, the individual amorphous filaments, coated with alumina, are converted to crystalline phases, primarily cordierite and alumina, with some mullite. The mullite is evident as "needle-shaped" crystals, as seen in the higher magnification photos (Figure 63 and Figure 64). Under conditions which challenge the stability of the PRD-66 microstructure, these needle-like formations are the first to degrade and holes begin to form in the centers of the individual yarn filaments. Neither sign of reaction was observed in either photo of the exposed candle. As a result of this analysis, it was concluded that the microstructure of the PRD-66 material was stable in the Karhula PCFBC environment. 


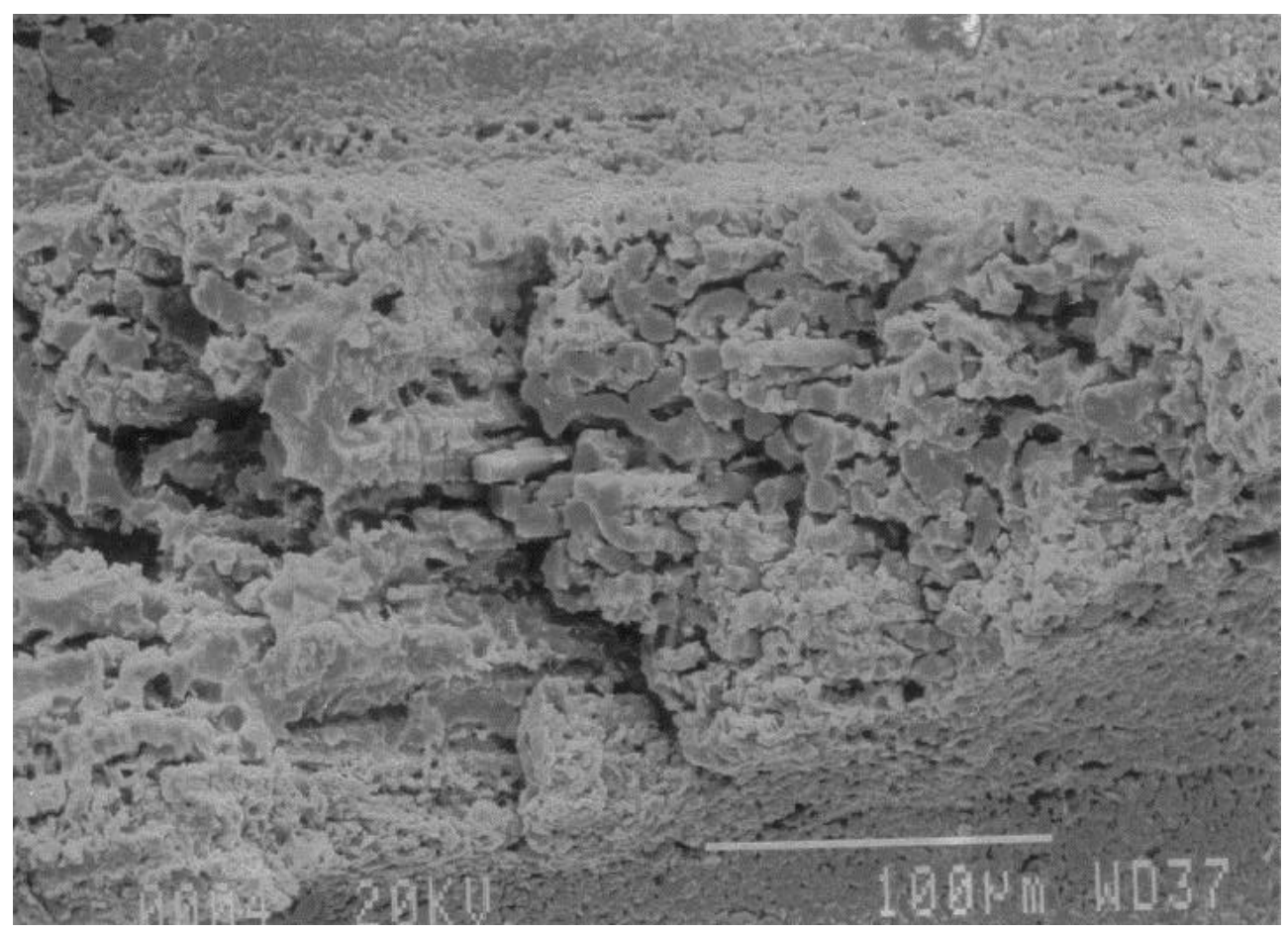

Figure 61 - UNEXPOSED CANDLE, individual "yarn bundle" (300X)

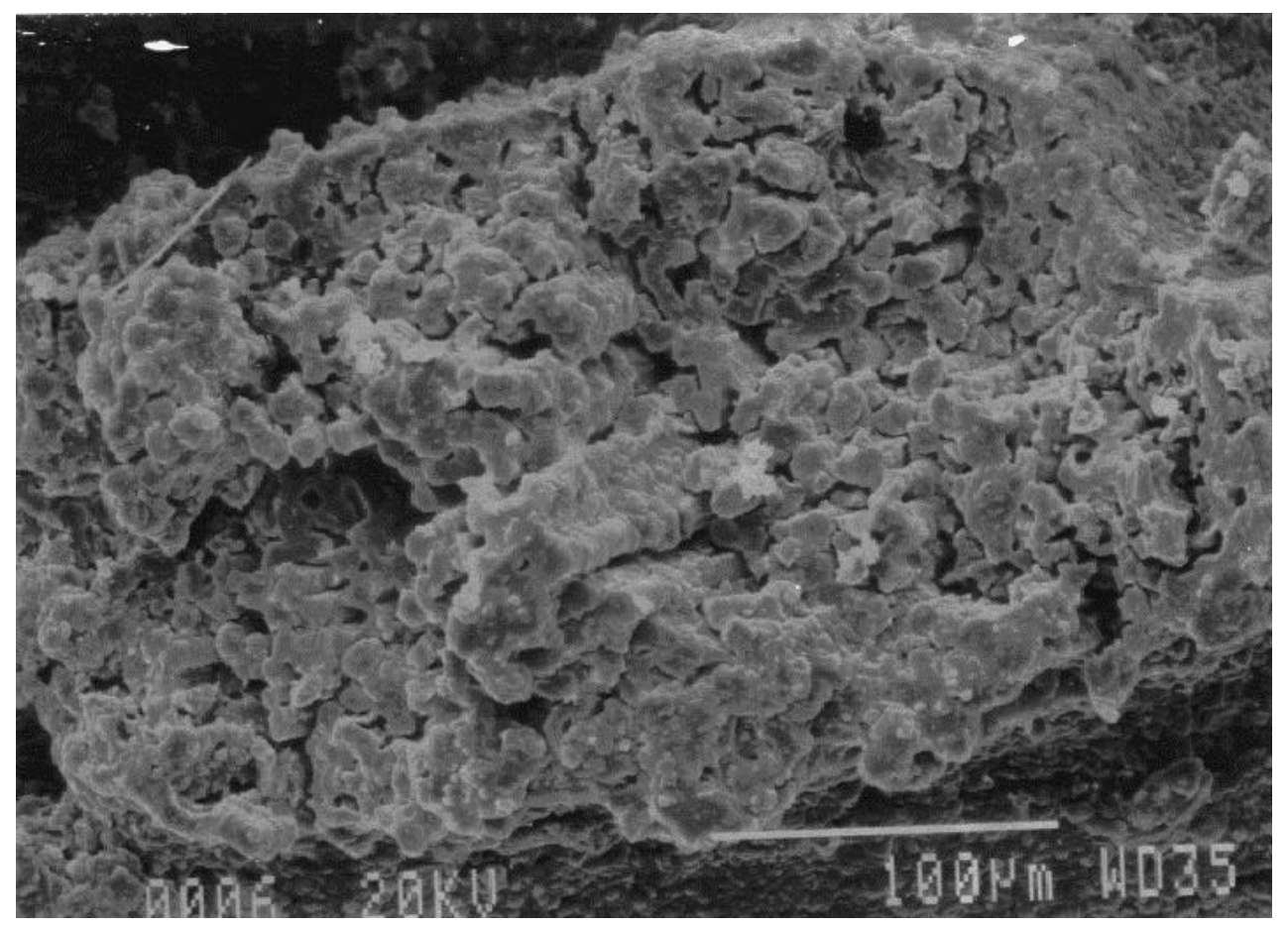

Figure 62 - EXPOSED CANDLE, individual "yarn bundle" (300X) 


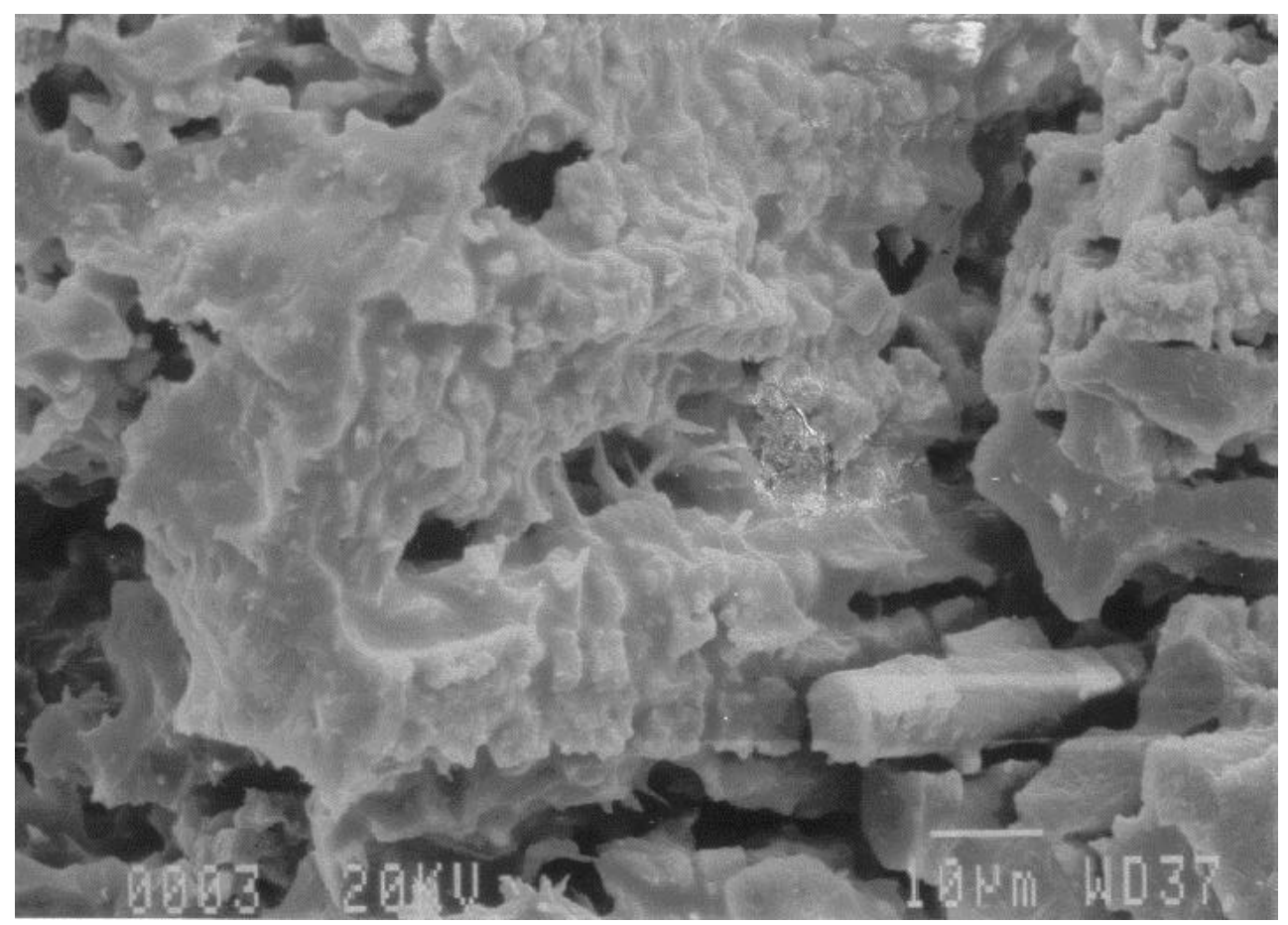

Figure 63 - UNEXPOSED CANDLE, individual "yarn bundle” (1,000X)

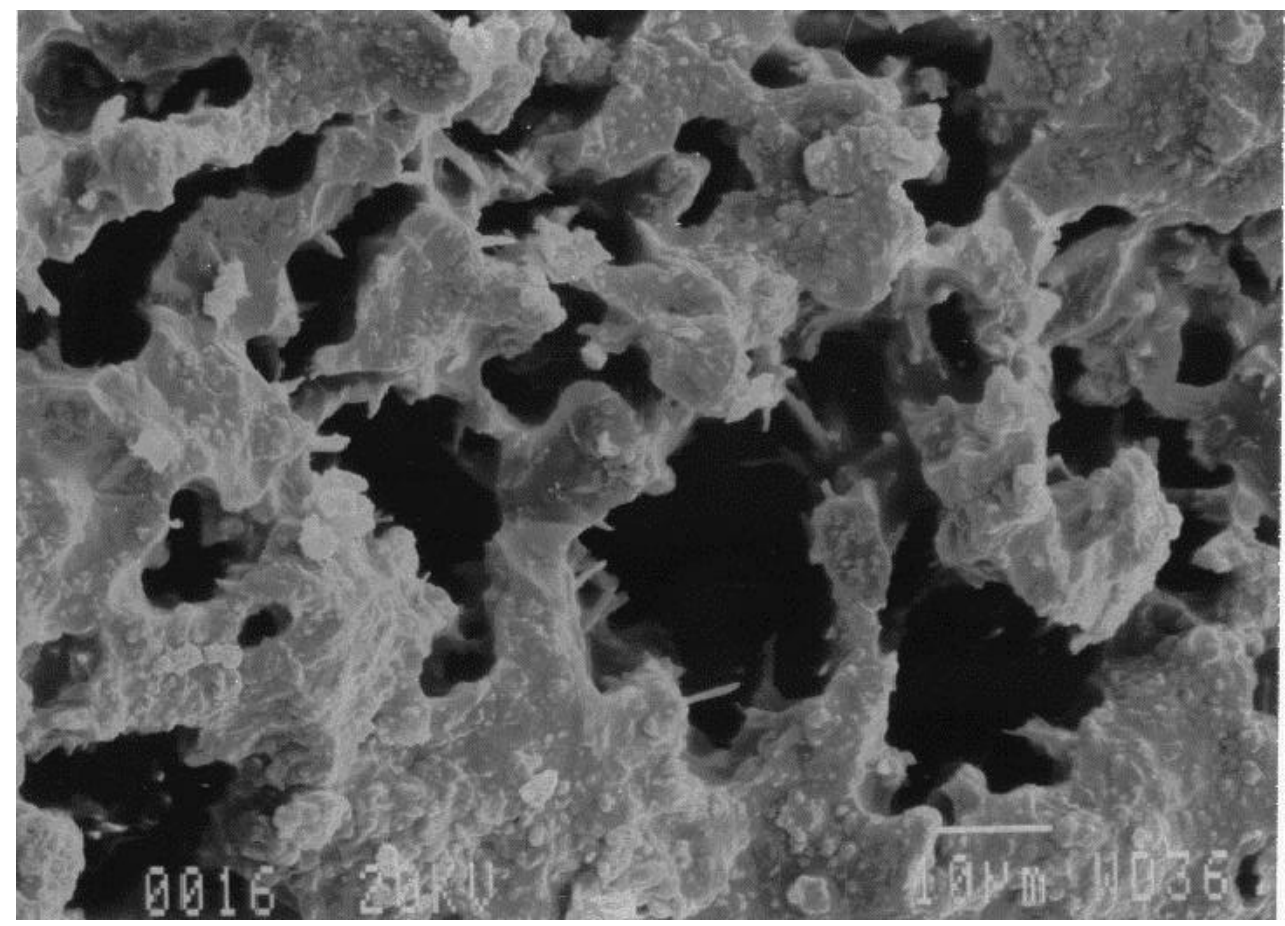

Figure 64 - EXPOSED CANDLE, individual "yarn bundle" (1,000X) 


\subsubsection{Diffraction Analysis of Karhula-Exposed and Unexposed Elements}

The stability of the PRD-66 material was further evaluated by qualitative x-ray diffraction (XRD). Specimens of candle \#576 (unexposed) and candle \#576 (581-hr exposure) were ground into powder and scanned from 5-90 degrees two theta. Both samples contained alumina, cordierite, mullite, and small amounts of cristobalite, in virtually identical amounts. The "exposed" material showed no evidence of any other crystalline phases that may have formed from a reaction of the PRD-66 with the PCFBC environment. The presence of coal ash in the "exposed" sample was not apparent since the material is not crystalline in nature. This analysis supports the visual SEM observation that the material was stable under the Karhula PFBC conditions.

\subsubsection{Strength Testing of Karhula-Exposed and Unexposed Elements}

As previously mentioned, two tested filter elements had been returned by Foster Wheeler to DLC. Candle \#577 had been exposed to 581 hours on coal. Candle \#591 had been exposed to 239 hours on coal and was broken at the flange when all candles were removed from the vessel for cleaning. 1-inch wide o-rings were sectioned from each candle and tested by o-ring diametrical compression. Average strengths and "load-to-failure" values are compared to unused candles as shown in Table 16. No apparent change in strength was observed.

\begin{tabular}{|c|c|c|c|c|c|}
\hline Unit No. & Condition & $\begin{array}{c}\text { Average } \\
(\mathbf{p s i})\end{array}$ & $\begin{array}{c}\text { Std. Dev. } \\
(\mathbf{p s i})\end{array}$ & Load-to-Failure (lbs.) & Samples \\
\hline C566 & Unexposed & 1087.6 & 80.8 & 41.5 & 11 \\
\hline C576 & Unexposed & 1256.2 & 64.7 & 45.6 & 6 \\
\hline C578 & Unexposed & 1352.9 & 65.2 & 48.1 & 5 \\
\hline C590 & Unexposed & 1076.1 & 47.8 & 47.4 & 6 \\
\hline C577 & Exposed-581hrs & 1246.6 & 49.9 & 50.0 & 6 \\
\hline C591 & Exposed-239hrs & 1315.0 & 103.9 & 57.0 & 6 \\
\hline
\end{tabular}

Table 16 - O-ring diametrical compressive testing of Karhula-exposed \& unexposed candles 


\subsubsection{Accelerated Life Testing of Karhula-Exposed Elements}

DLC received information from M.A. Alvin at W-STC concerning one of the Karhulaexposed PRD-66C filters that had been subjected to extended, accelerated life testing ${ }^{14}$. This filter failed after being subjected to 5055 accelerated pulse cleaning cycles (equivalent of $3109 \mathrm{hrs}$ of total PCFBC/PFBC total operation). The filter failed in the tapered region of the flange. Mary Anne did note that hairline cracks already existed in the area when it was received from Karhula.

The filters, which DLC received back from Karhula, were examined, trying to gain some insight into what may have caused the flange of Westinghouse's Karhula-exposed filter to fail. The flanges of filters \#577 \& \#591 were examined with a simple 10X magnifying glass. There were no visible cracks, however, we did notice several areas in the tapered area where the surface was slightly abraded. This brought up the concern that the damage may have been caused by the inside diameter edge of the filter-nut that supports the element. A more thorough discussion of this type of damage is contained in Section 3.8.2.2, on page 89 of this report.

The results of this test, however, were still extremely encouraging. The tested filter had identifiable hairline cracks in the flange before the test even began and still survived over 5000 accelerated pulse cleaning cycles!

The accelerated life testing of PRD-66C continued at Westinghouse with a new filter. In September 2000, they will begin testing a filter that had been exposed in Karhula, plus 1,300 hours in the Power Systems Development Facility in Wilsonville, AL. 


\subsection{Manufacturing 50 Filters (Task 5)}

To fulfill the requirement of Task 5, fifty filter elements were wound. Of those fifty, twenty-five were of suitable quality for use. The primary reasons for rejecting candles included "out-of-tolerance", "physical damage", "low weight or low alumina pickup" and "mandrel breakage". The most significant problem, however, was "winder malfunction"; $30 \%$ of all filters experienced some form of equipment failure during the winding of the slurry-coated fiberglass yarn. Most of the time, the repairs could be made immediately and the winding could be continued with no significant harm done to the filter element. However, $12 \%$ of all candles, which started the winding process, had to be rejected because the equipment malfunction either damaged them directly or the time needed for equipment repair would have allowed the incomplete, damp candle to dry out. During the Process Variable Experiment (Section 3.5.3:Process Variables Experiments (Subtask 4.3), page 42), a "safe period" of fifteen minutes was established for a process interruption; no adverse effects were observed, as long as the room humidity was kept within normal process limits. When any equipment repairs required more than fifteen minutes of downtime, the candle was rejected due to the increased risk of an undetectable delamination within the wall of the filter.

Compared to the Process Capability Study (Section 3.5.4) that produced thirty filters off one winder, more variability was observed in the data with regard to filter weights and alumina matrix pickup. This variability has been attributed to several factors associated with the use of an additional winder, its yarn tensioning system, and its sizing orifice, which strips the excess slurry from the yarn.

With regard to the backpressure of the finished filter elements, the nineteen elements made with the Type-C membrane were $3.6+/-0.9$ in-wg @ 50scfm. The nineteen elements made with the Type-M membrane were $6.4+/-1.0$ in-wg @ 50scfm. The reproducibility of the application of the particulate has improved with time.

All eight of the good PRD-66M filters were sent to the Wabash River Coal Gasification Repowering Project, near Terre Haute, Indiana. This facility uses an integrated gasification and combined cycle (IGCC) system to produce syngas for an advanced combustion turbine. It has a seven-unit slipstream where "experimental" filter elements can be tested. Partial funding for this program is provided by the DOE's Clean Coal Technology Program ${ }^{20}$. 


\subsection{Field Testing of Task 5 Filters (Subtask 3.4.1)}

Almost all of the PRD-66C filter elements made in Task 5 were sent the Power Systems Development Facility (PSDF) in Wilsonville, AL. These filters were incorporated into the field trials being conducted under DOE/FETC Contract DE-FC21-90MC25140 with Southern Company Services.

\subsubsection{PSDF Exposure History}

In April'98, Southern Company Services began their evaluation of PRD-66C Hot Gas Filters at their Power Systems Development Facility (PSDF) in Wilsonville, AL. The particulate control device (PCD) was a Westinghouse Advanced Particulate Filtration (APF) system being operated downstream of a Kellogg transport reactor, which was operating in coal combustion mode. Nominal operating conditions are shown in Table 17.

Table 17 - Power Systems Development Facility (PSDF) Nominal Operating Conditions

\begin{tabular}{|l|l|}
\hline Temperature & $1375^{\circ} \mathrm{F}\left(746^{\circ} \mathrm{C}\right)$ \\
\hline Pressure & $200 \mathrm{psig}$ \\
\hline Face Velocity & $5 \mathrm{ft} / \mathrm{min}$ \\
\hline Baseline Differential Pressure & 75 inches water gauge \\
\hline Inlet loading & 10,000 ppmw \\
\hline Total Number of Filter Elements & 91 (on two plenums) \\
\hline
\end{tabular}

The initial evaluation included three filters that had been exposed for 691 hours in Karhula (Page 63) and six new filters, which had been fabricated as part of Task 5 of this program (Page 77). In June, three additional new filters were added. After 1360 hours of exposure, no PRD-66C failures occurred. During this time, a sudden thermal excursion was responsible for the failure of all monolithic oxide filters, but the composite oxides and monolithic silicon carbides were unaffected.

In October'98 (TC04), a more severe thermal event occurred which completely fractured the clay-bonded silicon carbide filters on the lower plenum (see Figure 65); those on the upper plenum remained intact but their retained strength was highly suspect. ${ }^{13}$ None of the oxide composites (including PRD-66C) failed during this event, but one PRD-66C filter suffered localized membrane damage, apparently from the impact of a broken silicon carbide filter. All filter elements were removed. 


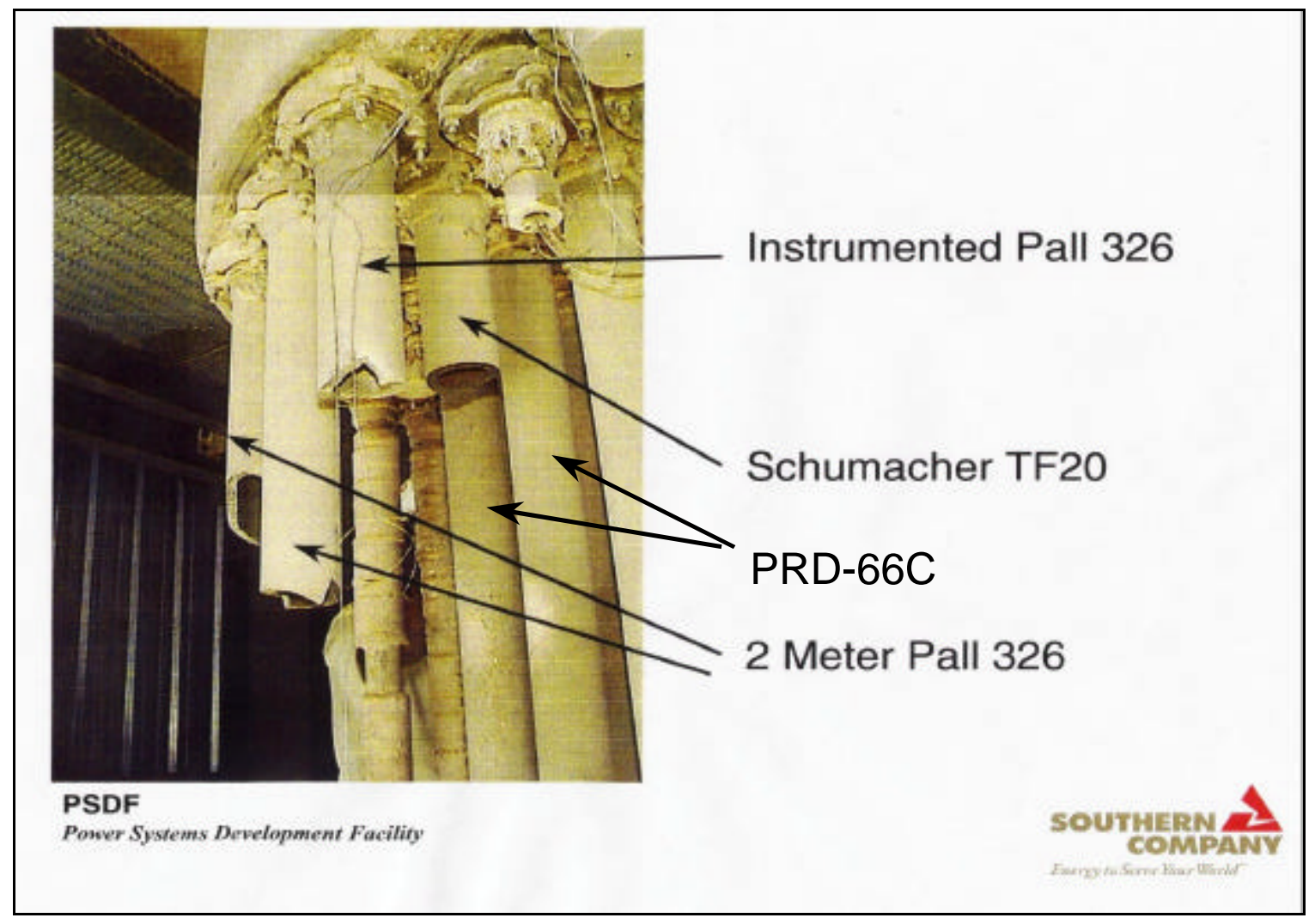

Figure 65 - Aftermath of Oct'98 thermal event in the lower plenum at the PSDF. ${ }^{13}$

In January'99, testing at the PSDF resumed with filter elements that had not been inservice during the thermal excursion. Of the fifteen (15) PRD-66C filters that were installed, two had some previous exposure and thirteen were from a new batch of filters manufactured in September and October of 1998. In early February, after the failure of another brand of composite-oxide filter, several of the PRD-66C filters were removed, checked for leaks and reinstalled. During reinstallation, filter \#C756 broke in the tapered region of the flange. Two additional PRD-66C filters were installed.

In late February'99, there were indications of a PRD-66C filter failure; the system was shut down and the PCD was opened. It was determined that the two most recently installed filters, and one neighboring filter, had broken at or near the base of the "filter nut" which holds the element in place. The decision was made to remove and inspect all PRD-66 filters. An additional filter broke at the base of the "filter nut" during it's removal and it was noted that two other PRD66C filters had two to three localized regions where the membrane had spalled off. No PRD-66C filters were reinstalled. 


\subsubsection{Failure Characterization and Investigation}

With additional financial support from FETC, a thorough investigation was begun to address the following failure modes that had been observed in the PRD-66C filters:

1. Localized spalling of the membrane

2. Breakage of the filter in the tapered region of the flange

3. Breakage of the filter at, or just below, the base of the filter nut

Since this effort related to the original scope of Task 3.4 (Failure Analysis and Correction), which focused on the Tidd field exposure, the new work has been included in this task. To differentiate it from that work, however, it was designated Task 3.4.1.

\subsubsection{Membrane Spalling}

A preliminary evaluation was conducted on the PRD-66C Hot Gas Filter, which had suffered localized membrane damage during the severe thermal upset that caused the failure of the clay-bonded silicon carbide filter. While starting up TC04 of the transport reactor, a $600^{\circ} \mathrm{F}$ thermal spike occurred. After the cool down of the vessel, one of eight PRD-66C filters (ID\#C649) had patches of membrane missing from one side. The focus of the initial investigation was to determine if the problem was similar to the "divots" which had caused the catastrophic failure of PRD-66 filters in Tidd-5 (Page 21). The following are the most significant observations made about filter C649:

a) damage was noticed in six obvious locations, the highest patch being 8 " below the flange and the lowest being 20 " below the flange, and one 1"-diameter patch in the middle of the filter, unlike Tidd-exposed filters which had "divot" down the entire length of the filter

b) all damage was on one side, unlike Tidd-exposed filters

c) no damage was present on any other filters, unlike all Tidd filters which had divots

Based on a comparison of visual characteristics, it is reasonable to conclude that the mechanism of membrane damage to C649 WAS NOT the same as that which caused divots in Tidd-5. The damaged observed, therefore, should not be characterized as a "divot". In addition, because the only filter effected was one of the "younger" elements, the defect mechanism could not be related to the length of exposure or basic material degradation. Evidence suggests that the 
damage mechanism was unique to $\mathrm{C} 649$, indicating a pre-existing defect in that one filter or an event that effected only that filter.

PSDF engineers accumulated significant amounts of information about the thermal event in TC04 and made the following observations concerning how the elements were effected:

a) All clay-bonded $\mathrm{SiC}$ on the lower plenum broke, and the ash cake was more sintered on this plenum, indicating a higher thermal excursion on the lower plenum.

b) The only clay-bonded $\mathrm{SiC}$ filter on the lower plenum that did not fall to the hopper was a Pall-326 filter, which was tethered by thermocouples; the broken segments protruded from the normal axis of the filter.

c) C649 was immediately adjacent to the tethered Pall-326; the membrane-damaged area was facing the protruding broken edges, which were at roughly the same vertical distance from the plenum

This investigation strongly suggests that the reason C649 was the only PRD-66C filter damaged, was because it was next to the tethered and broken Pall-326. According to PSDF engineers, approximately six hours after the initial thermal spike was observed, pieces of the silicon carbide filter began to appear in the hopper. The system continued to run for another twelve hours with air flowing. This scenario suggests that after the Pall-326 filter broke, its thermocouple tether allowed it to swing in the gas stream and hit the adjacent PRD-66C filter. During the eighteen hours which followed, multiple impacts would have occurred due to the turbulent flow conditions in the vessel. If, in fact, the obvious damage to PRD-66C649 was caused by the impact of an adjacent filter, it should be noted that this abuse did not result in the catastrophic failure of the filter element.

The two filters, which had patches of membrane missing following the February'99 run, were filters \#C740 (Figure 66) and \#C749 (Figure 67). It was noted that both filters had approximately $1 \mathrm{~mm}$ of the support wall missing along with the membrane and that the material spalled off evenly across a particular layer. This was very similar to the damage observed on C649, which was next to the broken $\mathrm{SiC}$ filter during the "fire". In reviewing the process information for these filters, it was determined that all of them had relatively low "alumina pickup", defined as the weight percent of alumina covering the fiberglass yarn. 


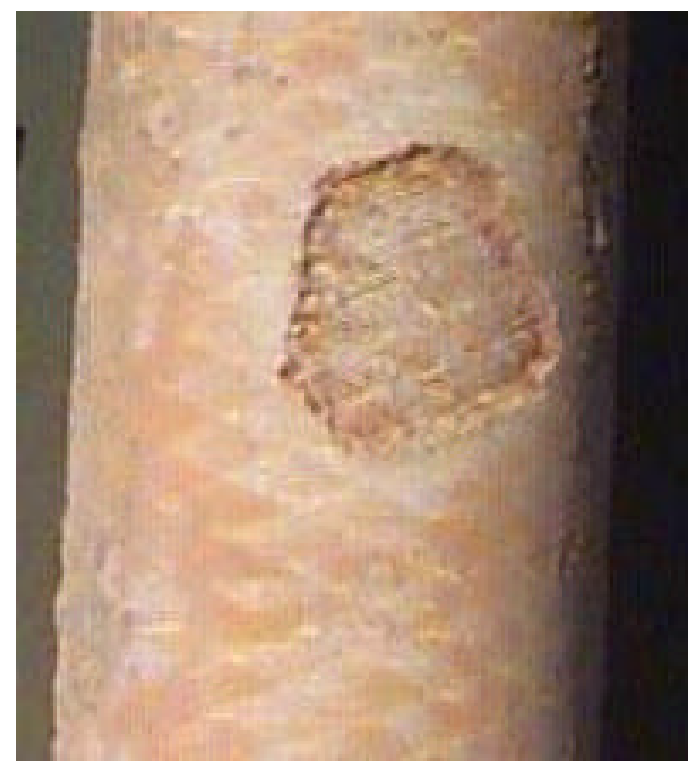

Figure 66 - C740 (T12), 18-19” from open end

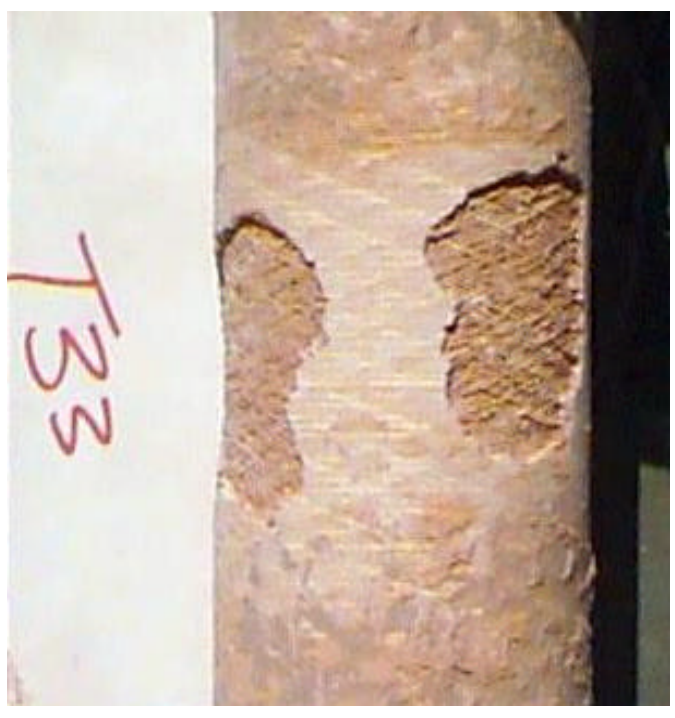

Figure 67 - C749 (T33), 12” from open end

These filters were more closely examined externally and internally when they were returned to HACI. In Figure 68 and Figure 69, the spalled patches are shown after a thorough vacuuming of the surface. It was apparent that the top three layers of filter body were removed, not just the membrane layer. There were no new patches of spalled membrane uncovered by the vaccuuming.

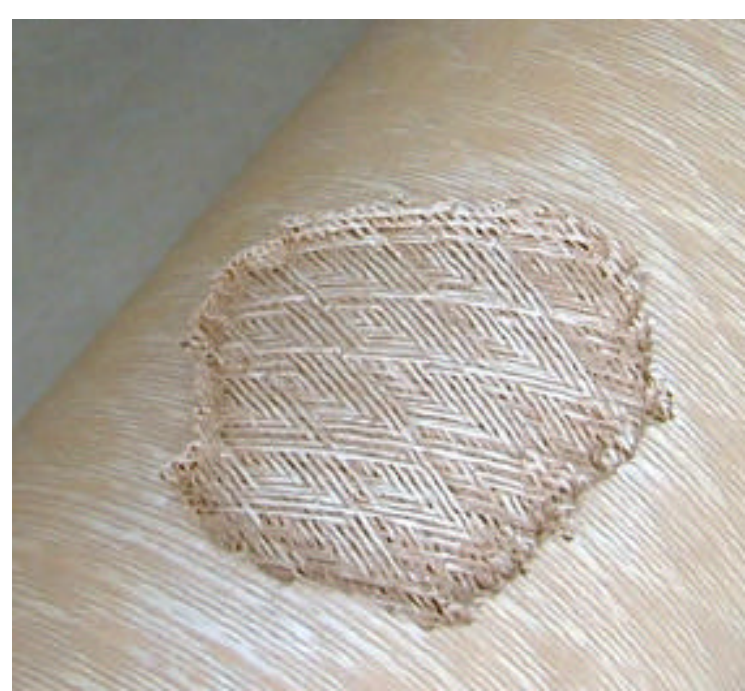

Figure 68 - Spalled area of Filter \#C740, 18'from open end, no other similar damage

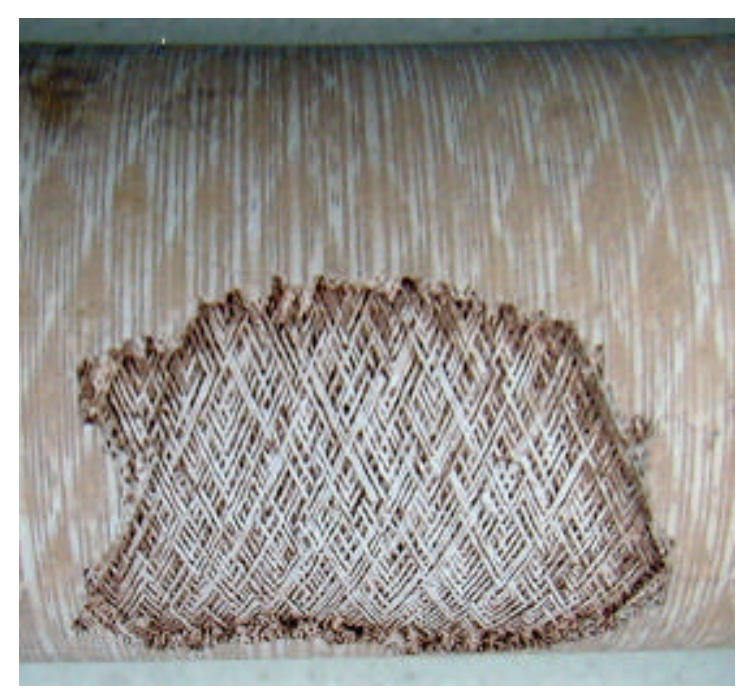

Figure 69 - Spalled area of Filter \#C749, 12” from open end, similar damage $90^{\circ}$ away. 
The damage on both filters was of a similar depth (1.5-2mm) and size (1.5-2" across and long). Filter \#C740 (Figure 68) had a single patch of missing membrane 18" from the open end. Filter \#C749 (Figure 69) had two similar patches, approximately $90^{\circ}$ apart, 12" from the open end. In addition, filter \#C749 had a very small dent in the membrane on the opposite side of one of the spalled area. There were no other spalled patches on the either filter. Four other filters were examined and several dents and small chips were observed on the surface, but no spalled patches. It was also noted that \#C749 had very fine cracks in the membrane entering and leaving the damaged areas.

Both C740 and C749 were cross-sectioned in the spalled area, and approximately one inch away. There was no evidence of any type of delamination or poor bonding. Attempts to remove additional pieces of the membrane were unsuccessful because the material was well adhered to the wall. The cross-section did reveal that the ash had plugged the wall beneath the damaged area; within one inch of the damage, however, the wall as clear of ash. This indicates that when damage to the membrane occurs, only localized blinding of the wall will result.

The most likely mechanism being considered for the spalling is mechanical damage. When spalled filter \#C749 (Figure 69) was being removed from the plenum, this author (ES Connolly) observed the following event:

- a workman was removing one of the bolts that holds the filter nut in place

- this workman was using a manual socket wrench

- as this workman rotated the handle of the wrench, the handle repeatedly made contact with the surface of the filter in one of the spalled areas

- each filter nut is held in place by several bolts, as he removed one of the other bolts the wrench was impacting the other spalled area

Although this observation does not prove that similar damage was inflicted on the filter during installation, it does suggest a reason why damage might occur at that particular location along the length of the filter. It was also noted that the spalled area of C740 (Figure 68) was 6" lower than the damage on C749, which suggests that a standard 6" socket extension may have been used during its installation.

This author also observed that a powered-version of the wrench was used by some workmen. Several new, small chips were noticed on the surface of the filters that had been removed with this wrench. 
These observations, coupled with the fact that no defect could be found with the material suggests the following:

- contact between tools and the filters during installation is a common occurrence

- in the case of $\mathrm{C} 740$ and $\mathrm{C} 749$, this resulted in spalling of the membrane

- if any of the other filters had similar impact, no significant damage resulted

This line of reasoning suggests that mechanical damage is necessary to initiate spalling of the membrane but other factors determine if spalling occurs, perhaps a property of the material, perhaps the severity of impact.

To study the effect of impact damage on the exposed PRD-66C filters, a Gardner "falling weight" impact tester was used to inflict a reproducible impact (ASTM D 2794). This device uses a $100 \mathrm{~g}$ weight with a $9 / 16$ " hardened steel ball at the impact-end. Because the damage that was observed at the PSDF suggested that multiple impacts were likely, a test procedure was designed to impact the surface of the filter at four locations within $1 / 2$ " of each other. The criss-cross pattern of the yarn was used to determine the exact positioning of the impacter and the weight was dropped from the same height each time. Six locations on each sample were tested. After the impact testing, the damage was not always very noticeable. Since filters are subjected to backpulsing when in service, the specimens were subjected to a stream of high-pressure air to dislodge any loose material after testing. Both C740 and C749 were tested, as well as C633, which had been subjected to 1,360 exposure hours with no sign of spalling.

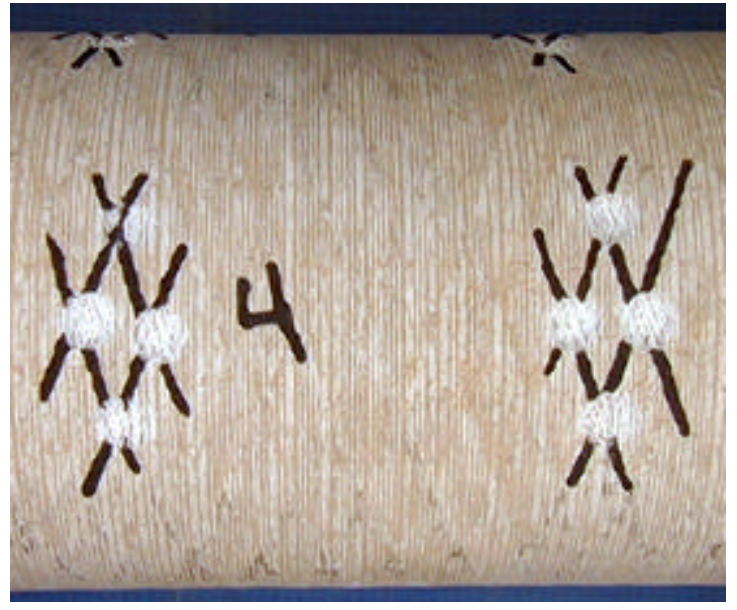

Figure 70 - Damage resulting from impact testing of Filter C633, Alumina Lot\#1, 1360 exposure hours

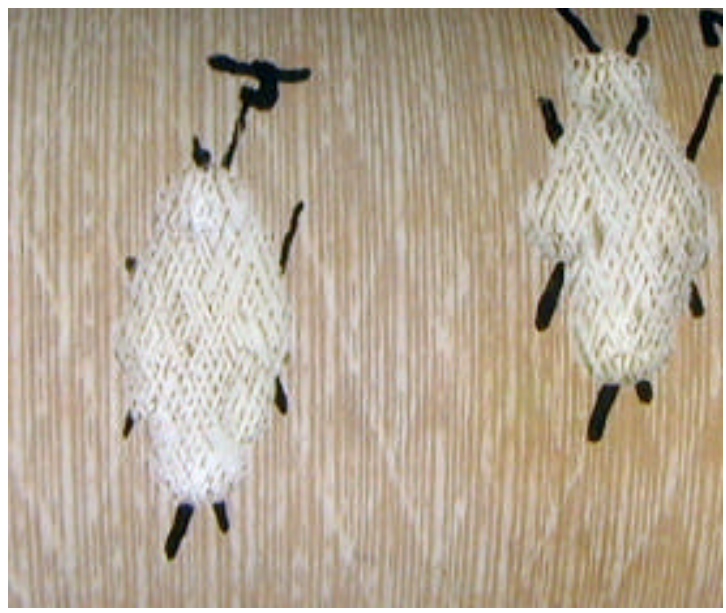

Figure 71 - Damage resulting from impact testing of Filter C740, Alumina Lot\#2, 636 exposure hours 
As shown in Figure 70, the damage done to C633 remained very localized at the points of impact. The damage done to C740 (Figure 71) was very different; cracks propagated between the impact sites and the entire area broke away. C749 had similar damage at half of the tested locations. Another difference observed was the depth of the damaged area, as shown in Table 18; the more recent filters suffered significantly deeper damage. This evaluation confirmed that the tester is capable of replicating membrane damage similar to that which occurs in the field. It should also be noted that Filter C633 had been made with a different lot of alumina powder than filters C740 and C749.

Table 18 - Damage Resulting from Impact Testing of Exposed Filters

\begin{tabular}{|c|c|}
\hline Filter Number & Damage Depth \\
\hline C633 & $0.9-1.2 \mathrm{~mm}$ \\
\hline C740 & $1.6-2.2 \mathrm{~mm}$ \\
\hline C749 & $1.5-2.4 \mathrm{~mm}$ \\
\hline
\end{tabular}

Using the Gardner impact tester, a controlled experiment was run to determine the effect of "Alumina Lot" and "Alumina Content" on impact resistance, as described in Table 19.

Table 19 - Design of Impact Damage Experiment

\begin{tabular}{|l|l|}
\hline \multicolumn{2}{|c|}{ Statistical Design of Impact Damage Experiment } \\
\hline Type of Experiment & $2^{2}$ Full Factorial \\
\hline Independent Variables & $\begin{array}{l}\text { Alumina Content } \\
\text { Alumina Lot }\end{array}$ \\
\hline Number of filters per "treatment" & 3 \\
\hline Number of locations tested per filter & 4 \\
\hline Number of impacts per location & 4 \\
\hline
\end{tabular}


The samples were prepared in the following way:

1. Test specimens were selected from ACI's inventory of PRD-66 Hot Gas Filters. Some of these filters had already been cut up and tested for other purposes.

2. Three specimens were chosen which met the criteria for each of the $2^{2}$ factorial catgories:
a) High alumina - Alumina Lot 1
b) High alumina - Alumina Lot 2
c) Low alumina - Alumina Lot 1
d) Low alumina - Alumina Lot 2

3. Specimens were marked at four locations for testing, and each location was labeled.

4. Each "location" was defined as the corners of the "diamond-shaped" yarn pattern of the PRD-66 wall-structure. Each "location" was indicated by magic marker.

5. All specimens were weighed before testing.

The test was performed in the following manner:

1. The impact tester was dropped from a predetermined height and struck the specimen at the corners of the "diamond-shaped" outline at one of the specified locations.

2. A high-pressure air gun was used to blow off any loose material.

3. The specimen was weighed and the weight loss was noted.

4. The second, third, and forth locations on the specimen were tested.

5. After the forth test, the depth of each damaged area was measured and a rating was assigned to the surface damage. Figure 72 illustrate the rating scale for surface damage. Ratings of " 2 " or " 3 " indicate that material spalled off between two or three impact points, respectively. 


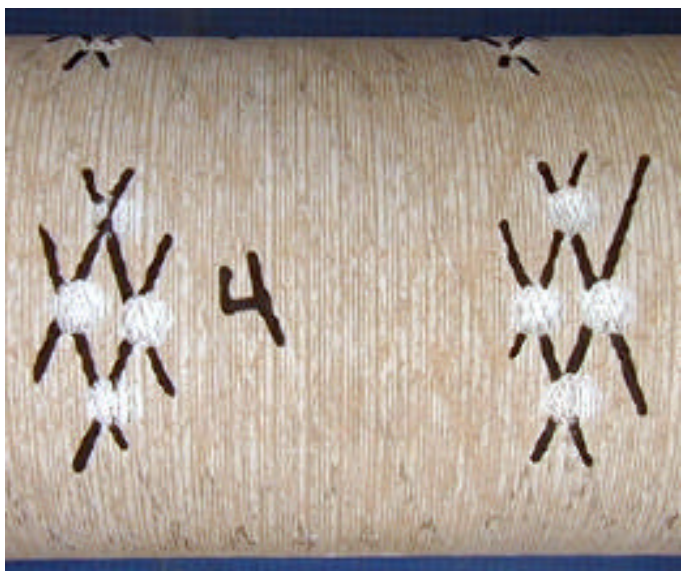

Figure 72 - Surface Damage Rating = 1

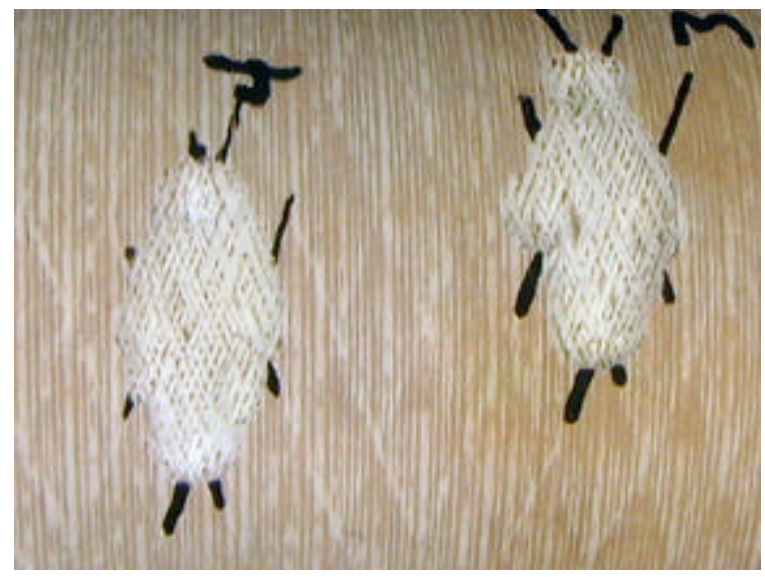

Figure 73 - Surface Damage Rating = 4

The resultant damage, therefore, was evaluated in three ways - the severity of spalling, the depth of the damage and the amount of material removed. It turned out that the data collected on "weight removal" was not useful because it was effected by both spalling and depth. With regard to spalling, Alumina Lot \#2 did not perform as well as Alumina Lot \#1, as indicated in Table 20. The Alumina Content did not seem to effect the spalling, but it was noted that some samples made with Alumina Lot \#1 performed dramatically better than others (note the high standard deviation).

Table 20 - Effect of Alumina Content and Lot on Surface Damage Rating

\begin{tabular}{|l|l|l|l|l|}
\hline \multirow{2}{*}{ SURFACE DAMAGE RATING } & \multicolumn{2}{|c|}{ Alumina Lot 1 } & \multicolumn{2}{c|}{ Alumina Lot 2 } \\
\hline & Mean & Std.Dev. & Mean & Std.Dev. \\
\hline Alumina Content $>54 \%$ & 1.75 & 1.3 & 3.5 & 0.5 \\
\hline Alumina Content $<52 \%$ & 1.75 & 1.1 & 3.08 & 0.4 \\
\hline
\end{tabular}

With regard to the depth of damage, specimens with both a high alumina content and Alumina Lot\#1 were not damaged as deeply as the others, as indicated in Table 21. In general, filters made with Alumina Lot \#1, having more that 54\% alumina showed less damage as a result of impact. 
Table 21 - Effect of Alumina Content and Lot on Depth of Damage

\begin{tabular}{|c|c|c|c|c|}
\hline DEPTH OF DAMAGE $(\mathbf{m m})$ & \multicolumn{2}{|c|}{ Alumina Lot 1 } & \multicolumn{2}{c|}{ Alumina Lot 2 } \\
\hline & Mean & Std.Dev. & Mean & Std.Dev. \\
\hline Alumina Content $>54 \%$ & 0.69 & 0.25 & 1.56 & 0.22 \\
\hline Alumina Content $<52 \%$ & 1.33 & 0.33 & 1.52 & 0.22 \\
\hline
\end{tabular}

Impact testing of the filters made with both lots of alumina showed that the filters made with "alumina lot \#2" were more likely to have pieces of the surface spall off. The alumina content, however, had no detectable effect on the spalling but filters with low alumina content did suffer deeper damage. In reviewing the data, very unusual results had been obtained during the testing of filter \#C684 (made with "alumina lot \#1"). Unlike other filters made with the same lot of alumina, it exhibited significant spalling in all locations tested. A polished cross-section of the filter wall revealed a very slight delamination less than $1 \mathrm{~mm}$ below the outside diameter surface. It was determined that during the last twenty minutes of the winding of this filter several yarnbreaks had occurred. Filter \#C749, which spalled at the PSDF, also had a winding interruption late in the run. This filter had no visible delamination, however, during the period of an interruption, the alumina slurry-coated yarn on the surface could dry slightly and not bond as well to the next layer of yarn.

It has been concluded, therefore, that PRD-66C filters are more susceptible to membrane spalling as a result of impact damage if:

a) they were made with "alumina lot \#2"

b) they experienced a winding interruptions late in the winding process

HACI has also suggested to the PSDF that a piece of rubber or foam tubing be slid over the handle of the manual socket wrenches, which are used to install ceramic candle filters. We requested that the "power wrench" not be used. 


\subsubsection{Flange Fracture in the Tapered Region}

As previously mentioned in Section 3.8.1, the one of PSDF's PRD-66C filters broke in the tapered region of the flange during reinstallation $n$ the beginning of February'99. A similar fracture had been noticed by M.A. Alvin at Siemens Westinghouse Power Corportation during the “Accelerated Life Testing" of a PRD-66C filter, which had originally been exposed in Karhula. ${ }^{14}$

The filter which broke during reinstallation was C756 (T-31); the flange portion is shown in Figure 74. All production records for this filter were reviewed. All specifications were nominal except for the fact that it had the longest flange of any filter supplied to Wilsonville $-27.8 \mathrm{~mm}$ (specification $27+/-1 \mathrm{~mm}$ ). The location of the break suggested that the it may have resulted from the pressure of the supporting edge on the inside diameter of the filter nut.

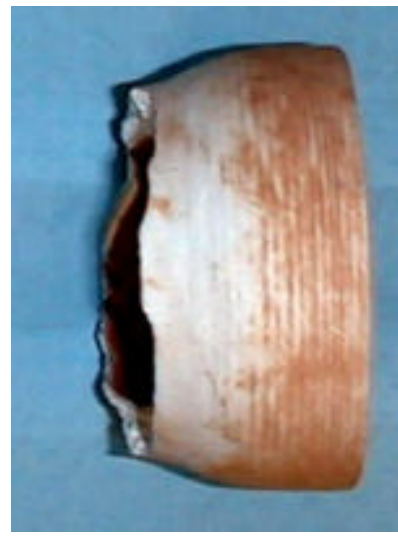

Figure 74 - C756 (T-31) broke during reinstallation

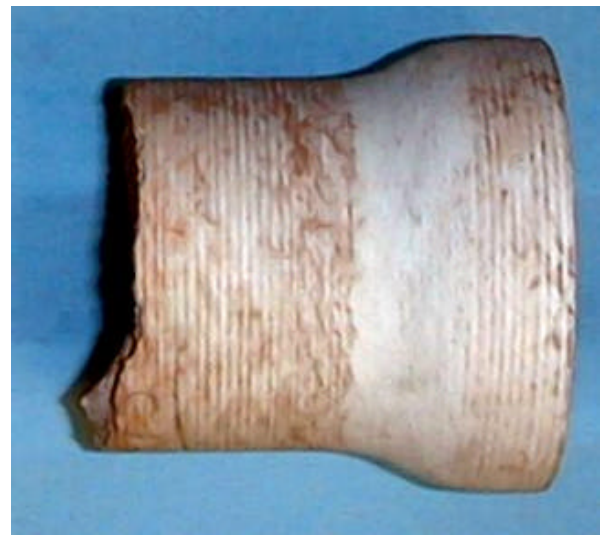

Figure 75 - C750 (B-49) with fine crack at base of tapered region of flange

One of the filters that broke catastrophically during tests at the PSDF had an additional crack detected at the base of the flange, as shown in Figure 75. This crack was very fine and extended around half of the circumference. The length of the flange was measured at several locations and found to be nominally $27.2 \mathrm{~mm}$ long. The side that was cracked, however, measured $27.6 \mathrm{~mm}$ long at some locations. An optical comparitor was used to better define the part geometry. Given the contours of the flange area, the descriptions chosen to define the shape are detailed in Figure 76. 


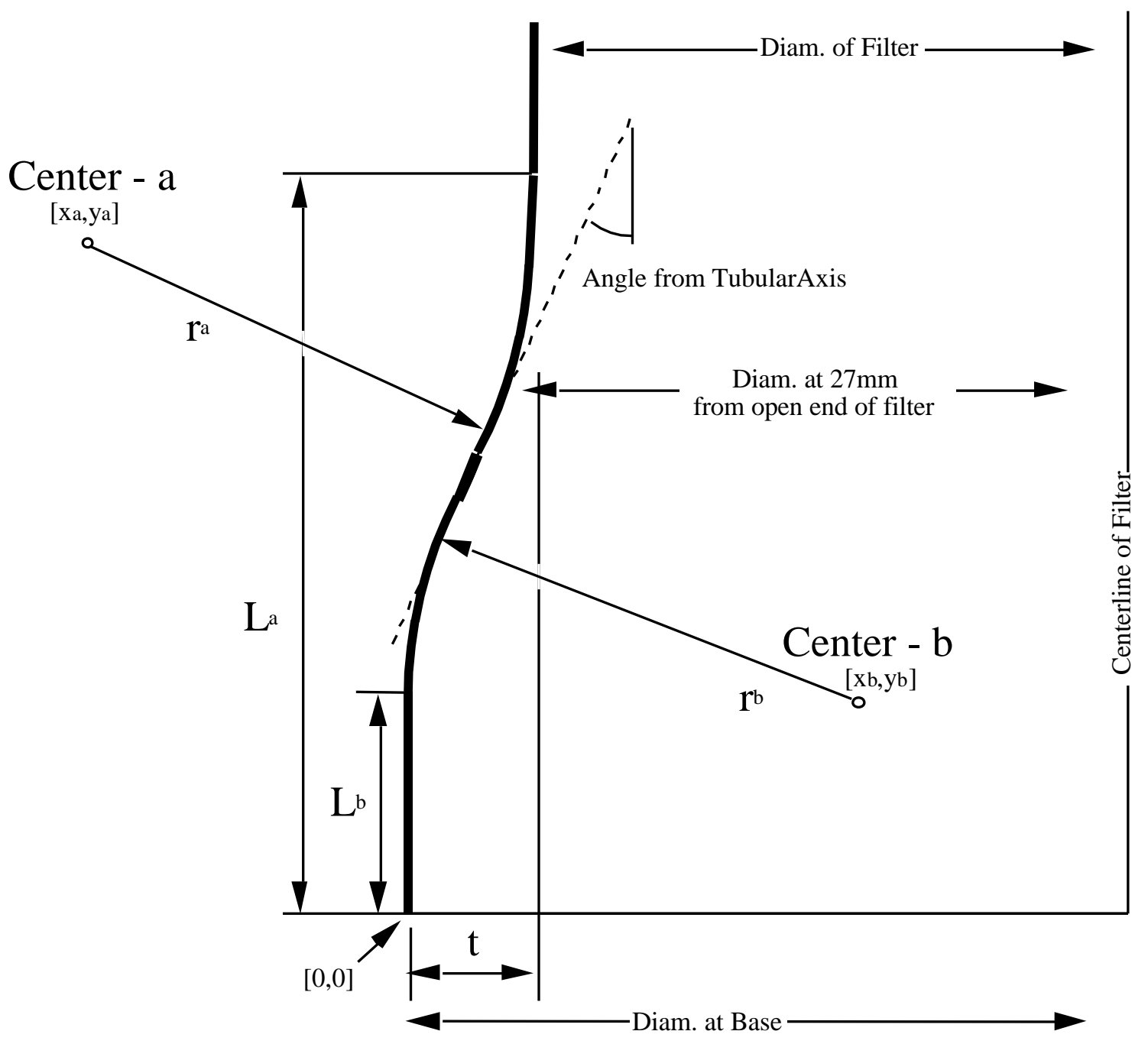

Figure 76 - Description of flange contours as determined by Optical Comparator

Two additional "exposed" filters had a visible crack in the same vicinity. Comparisons were made between the geometry of these flanges and those of other "exposed" filters that showed no signs of damage to the tapered region. The most significant observation made was that flanges, which were damaged in the tapered region, had a wider "diameter at $27 \mathrm{~mm}$ from open end of filter". Three possible mechanisms that could cause the diameter to be larger in this region

- overall larger diameter flange

- more shallow angle on the taper

- longer $\mathrm{L}_{\mathrm{b}}$ 
Comparisons of those dimensions did not give one consistent answer. The possibility that anyone of these problems could exist had to be addressed, but a better understanding of our customer's requirements was needed.

Since flange diameter and length are part of the Westinghouse specifications, an investigation was conducted to clarify how the specifications for the flange had evolved. When PRD-66 filters were first tested at the Westinghouse Science and Technology Center in mid-1993, breakage of the flange in the tapered region was a chronic problem. At a meeting of W-STC and DuPont Lanxide Composites personnel the following modifications were agreed upon:

1. The flange region of the filter would be strengthened by the infiltration of additional alumina for approximately 2.5 " from the open end.

2. The inside edge of the filter nut would be rounded to minimize the stress put on the tapered area of the filter nut.

3. Because the rounding of the edge of the filter nut would cause the flange to sit lower, the flange would to be lengthened by about $3 \mathrm{~mm}$.

The changes were incorporated into the filters and the modifications were made to the filter nut used by W-STC. No flange failures were observed with the filters evaluated in the high temperature high pressure (HTHP) test conducted by Westinghouse. During the Karhula test, one filter broke in the tapered region of the flange, during removal of the filter from the plenum. One Karhula-exposed filter, which was returned to Westinghouse for "extended accelerated life testing" had a fine crack in the tapered region, then broke during testing. At the PSDF, one filter broke in the tapered region and two filters had visible cracks in the same area following exposure.

Mary Anne Alvin at Siemens Westinghouse was asked to determine if the filter nuts used in Karhula and at the PSDF had the modified, rounded edge, similar to the HTHP test rig. Her investigation uncovered the fact that all the filter nuts used in the field trial were the "standard" design, and did not have the rounded inside diameter edge. The use of the standard mounting arrangement would have caused two significant problems for the PRD-66 filter:

1. The sharp edge of the filter nut would tend to concentrate the mounting forces in the most vulnerable region of the flange. 
2. Because the flange had been lengthened by $3 \mathrm{~mm}$ to accommodate a rounded-edge filter nut, the tapered region of the flange would be under excessive compressive force. The problem would be magnified if the mounting technique required the installation of the filter nut to a specific position (as in the case for the PSDF system).

Continued discussion revealed that it was not practical for Siemens Westinghouse to supply custom filter nuts to their customers for use with PRD-66 filters. PRD-66 filters needed to be able to fit into the "standard design". The diagram shown in Figure 77, which described how AlliedSignal Composites measures the length of the flange, was faxed to Siemens Westinghouse and reviewed by their staff. Based on the method ACI uses to measure the flange and the method SW recommends for tightening the filter nut, they concluded that the PRD-66 flange should be shortened by $2 \mathrm{~mm}$. The revised specification should be $25 \mathrm{~mm} \pm 1 \mathrm{~mm}$, instead of $27 \mathrm{~mm} \pm 1 \mathrm{~mm}$.

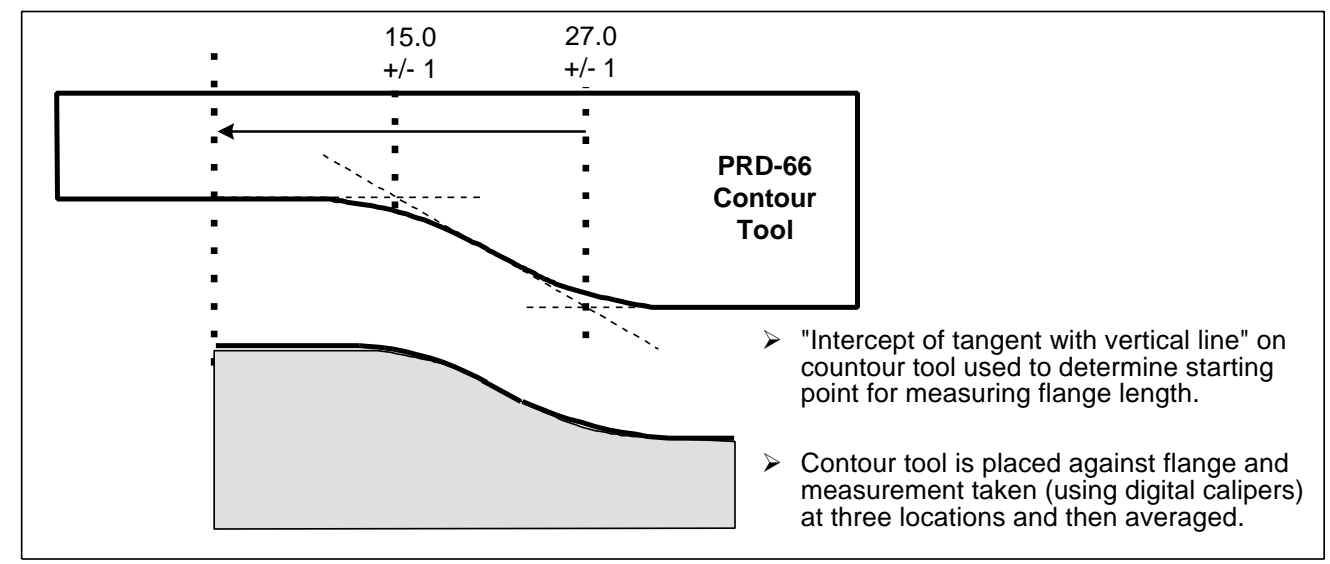

Figure 77 - PRD-66 Flange Measurement Technique

To address the issue of achieving a better fit of the PRD-66 filter into the more common mounting systems, the prospect of modifying the geometry of the flange was investigated. Figure 78 is an example of a standard flange, after it has been over-wrapped with the membrane yarn. To demonstrate the feasibility of reshaping the flange, the filter shown in Figure 79 was modified during course of the winding process. A significantly sharper radius was achieved at the base of the flange. One problem, which was encountered, however, involved the application of the membrane yarn. As the winding proceeded up the sharp angle, it backslid on top of itself. To achieve the results shown in the photo, the winding of the membrane yarn was stopped, the excess yarn removed, and the yarn guide manually positioned to resume winding. Since the presence of the membrane yarn is not critical around the flange, it would be feasible to end the membrane winding at the base of the flange, and then fill the surface of the flange with the type-C particulate membrane filler. 
Additional flanges were fabricated using various techniques. The specimens were then cross-sectioned to determine if any internal distortions or delaminations occurred; one particular technique gave the best results.

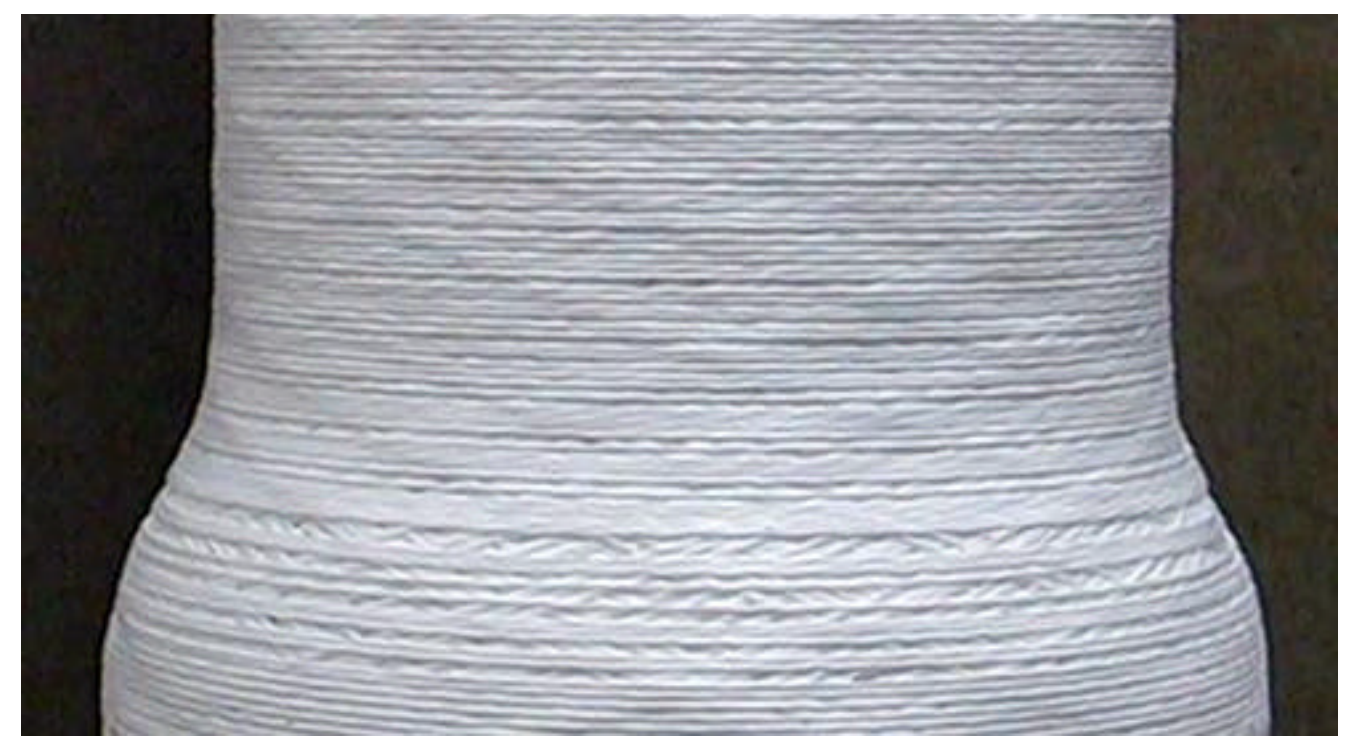

Figure 78 - Standard-shape PRD-66 flange

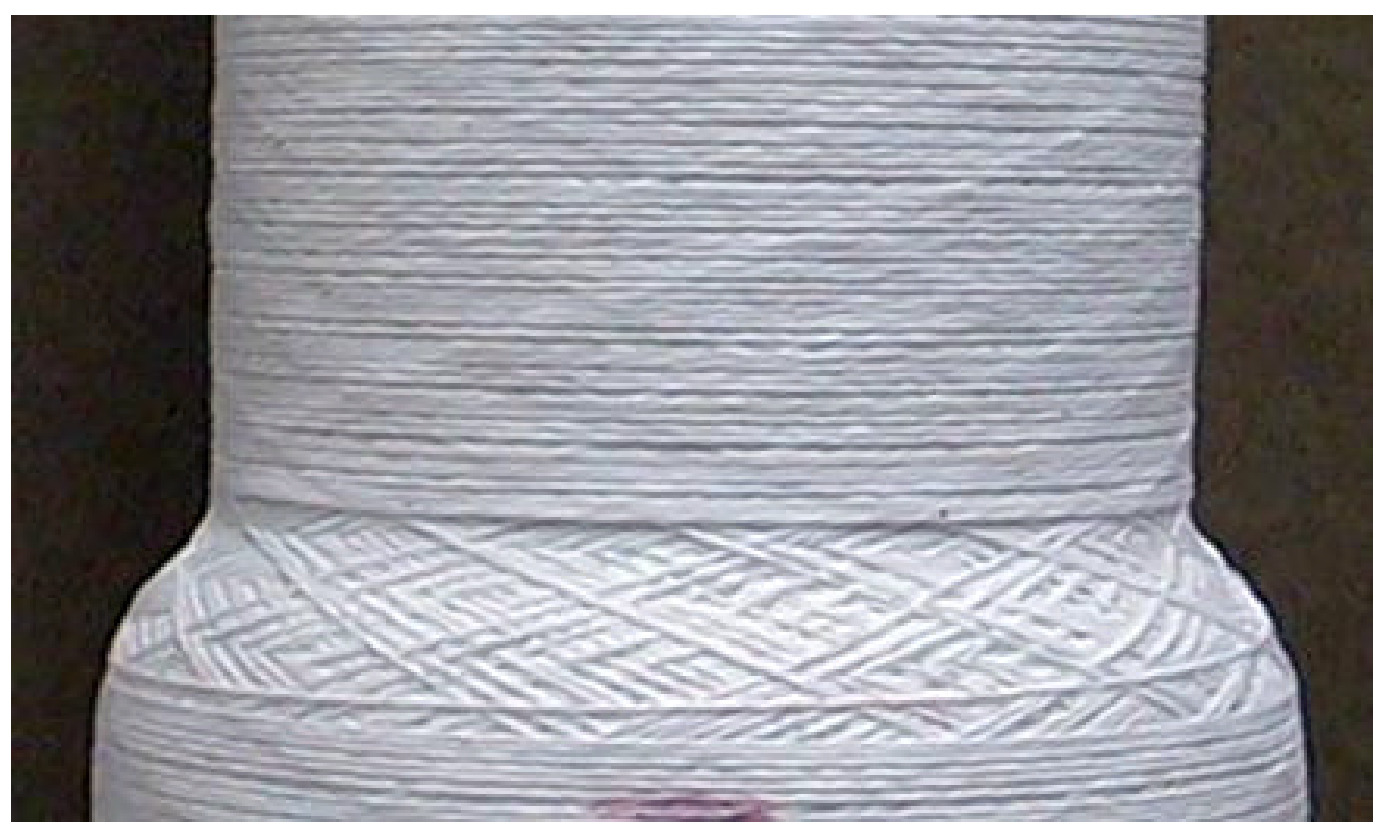

Figure 79 - Modified PRD-66 flange using manual techniques

Besides the obvious advantage of creating a shape that is better suited for the "typical" filter mount, the accuracy with which the flange length can be measured will be greatly improved. A similar automated technique could be incorporated into the design of the prototype filter winder. 


\subsubsection{Fracture Just Below the Flange}

After the February'99 run at the PSDF had been stopped, the PCD was opened up and the following observations were made during the removal of the PRD-66C filters from the plenum:

1. The filters which broke in-process and during removal all broke at the base of the filter-nut or just below the filter-nut.

2. There was apparently no "top gasket" on one of the three broken filters in the lower array. The other two filters that broke on the lower plenum were adjacent to this one.

3. All filters required a significant amount of "wiggling" to pull the flange out of the tube sheet.

Examinations were conducted on each of the broken filters, as well as the unbroken filters to determine if they showed signs of similar damage. Three filters broke exactly at the base of the filter-nut, as shown in Figure 80: C731, C750 and C752. All production specifications were nominal for these filters except that the alumina content was at the low-end of the acceptable range. It was also noted that these filters broke at the edge of the "infiltrated zone" (the region where the flange is reinforced by the addition of more alumina). A very fine crack was detected in the same area on Filter C749 (Figure 81). The crack was visible around half the circumference, at the lower edge of the "clean zone" on the flange.

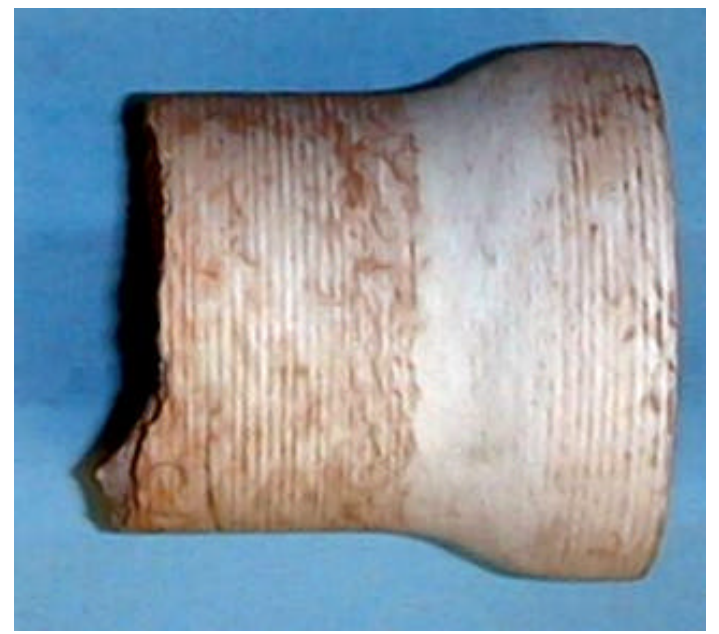

Figure 80 - C750, similar to C731 and C752

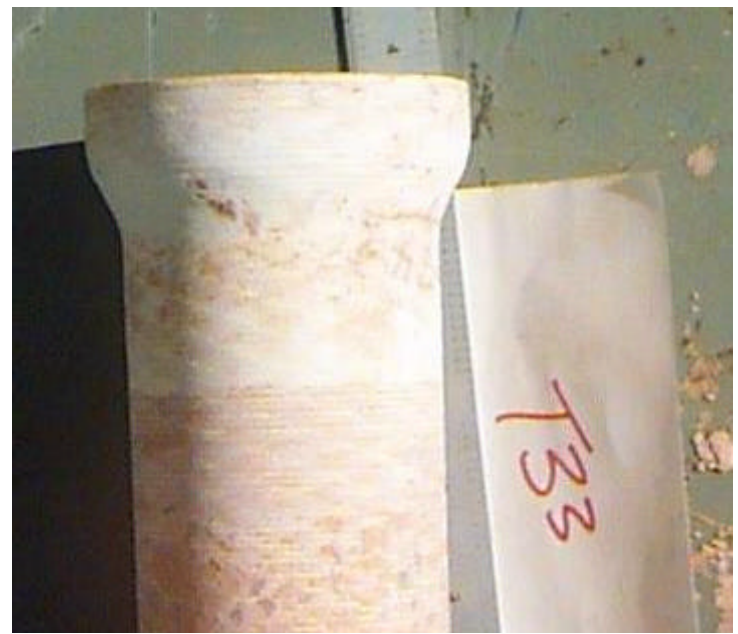

Figure 81 - C749 (T33), flange end 
During the examination of exposed filters, several small localized damage sites were detected on the filters. They were generally less that $1 / 8$ " wide, less than $1 / 4$ " long, and less than $0.5 \mathrm{~mm}$ deep. One filter had a noticeably different type of damage - Filter C752, which broke at the base of the filter nut as it was being removed from the plenum. 11/4" below the break was a rather unusual chip (see Figure 82; it was $1 \mathrm{~mm}$ wide and $2 \mathrm{~mm}$ deep at one end, then tapered up to the surface at the other end. Because the chip was full of ash, the damage must have occurred before the ash-feed to the PCD was turned off, either prior to installation, during installation or during operation. The PSDF engineers were not able to suggest a mechanism for the damage. It is unknown if this type of damage contributed to the failure of the filter.

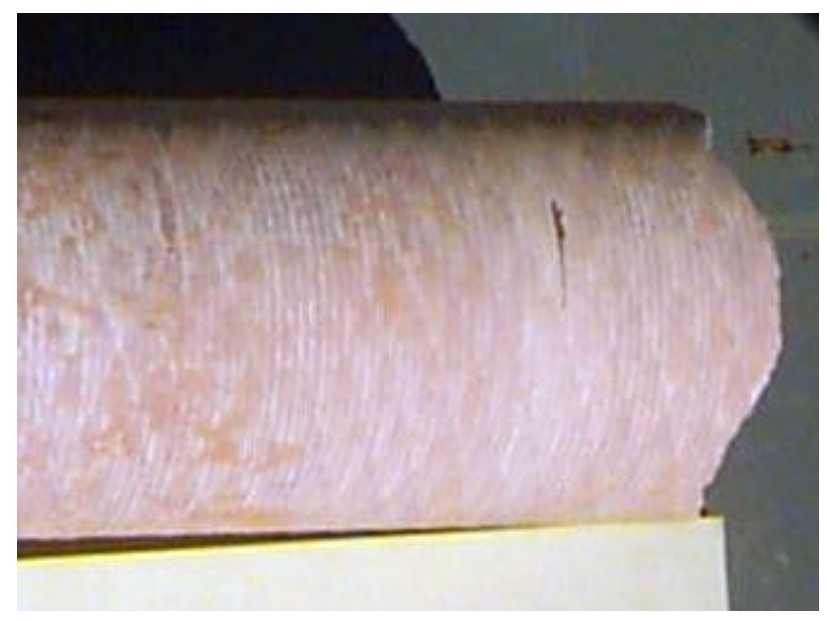

Figure 82 - Broken end of Filter C752, with deep chip 11/4" below the break

Filter C745, which had been adjacent to $\mathrm{C} 750$ and $\mathrm{C} 731$, had a distinctly different type of fracture. It broke 2" below the filter nut in an angular fashion that followed the winding pattern. The shape of this break resembled the type of damage typically seen if the filter is subjected to a severe impact, such as being dropped. A similarly-shaped filter break occurred at the PSDF when the filter nut of C633 had to be removed by hitting it with a hammer. This resemblance strongly suggests that $\mathrm{C} 745$ was broken by one of its falling neighbors. This filter also had "low percent alumina pickup", similar to C750, C731, and C759. 


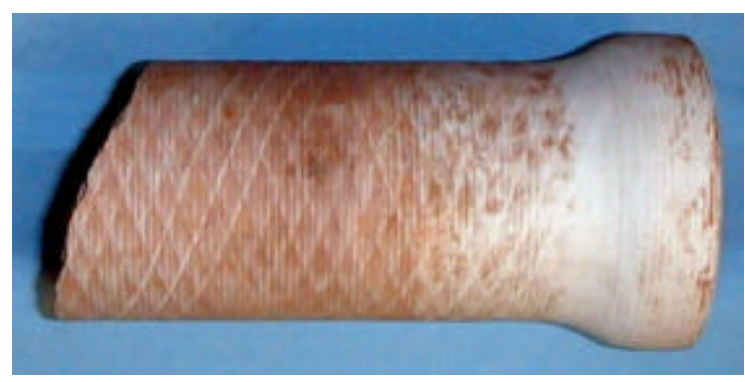

Figure 83 - C745 (B-54)

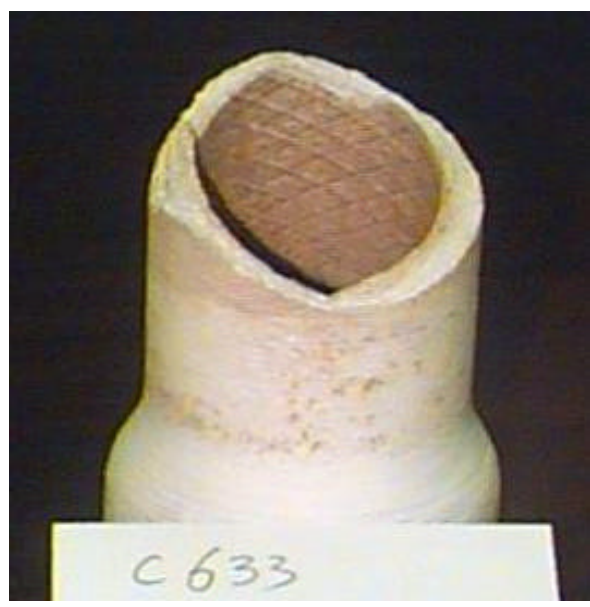

Figure 84 - C633, broke removing filter-nut

The focus of the remainder of the investigation was placed on the failures "at the base of the filter-nut". Historical data on all PSDF-exposed filters was analyzed, with particular attention to the failure mode, the exposure history, and the "percent alumina pickup". These filters had three things in common:

1. all of the broken filters were part of a later batch of filters shipped to the PSDF

2. "percent alumina pickup" on the low side of the nominal range

3. broke at the edge of the "infiltrated zone" of the flange

Additional factors may also have played a role in the failure of filters that broke at the base of the filter nut:

1. The lack of a gasket on the top of the flange could have resulted in the insecure mounting of filter C731. More significant vibration problems may have resulted, which would have concentrated the stresses at the base of the filter-nut.

2. The wiggling necessary to remove some filters from the tube sheet also concentrates stresses at the base of the filter-nut. Thus, filters that are held more firmly in place by ash are subjected to more stress during removal.

3. PRD-66C filters vibrate when hanging in the tube sheet, as a result of their low, axial modulus $(0.35 \mathrm{msi})^{14}$. Stresses would be concentrated at the base of the filter-nut. 
These potential problems all relate to characteristics of the filtration system. Addressing these issues was beyond the scope of this contract. The approved scope of work focussed on reasons why this particular assortment of filters failed. In addition, because the broken filters had been exposed for less than 636 hours, while others had achieved over 1,300 hours, "exposure to the PFBC environment" was not considered as a contributing factor in the failures.

In an effort to determine when significant changes may have occurred, o-rings were cut from a variety of filters produced over a two-year period. Figure 85 displays the data in the order in which the filters were fabricated (identification numbers are assigned sequentially) and four changes in the raw material supply were noted. The only raw material change that appeared to be significant was the use of a new lot of alumina powder (A-17 grade, made by Alcoa). The "questionable lot" of filters shipped to the PSDF contained two filters made with the first lot of alumina powder and twenty-six filters made with the second lot.

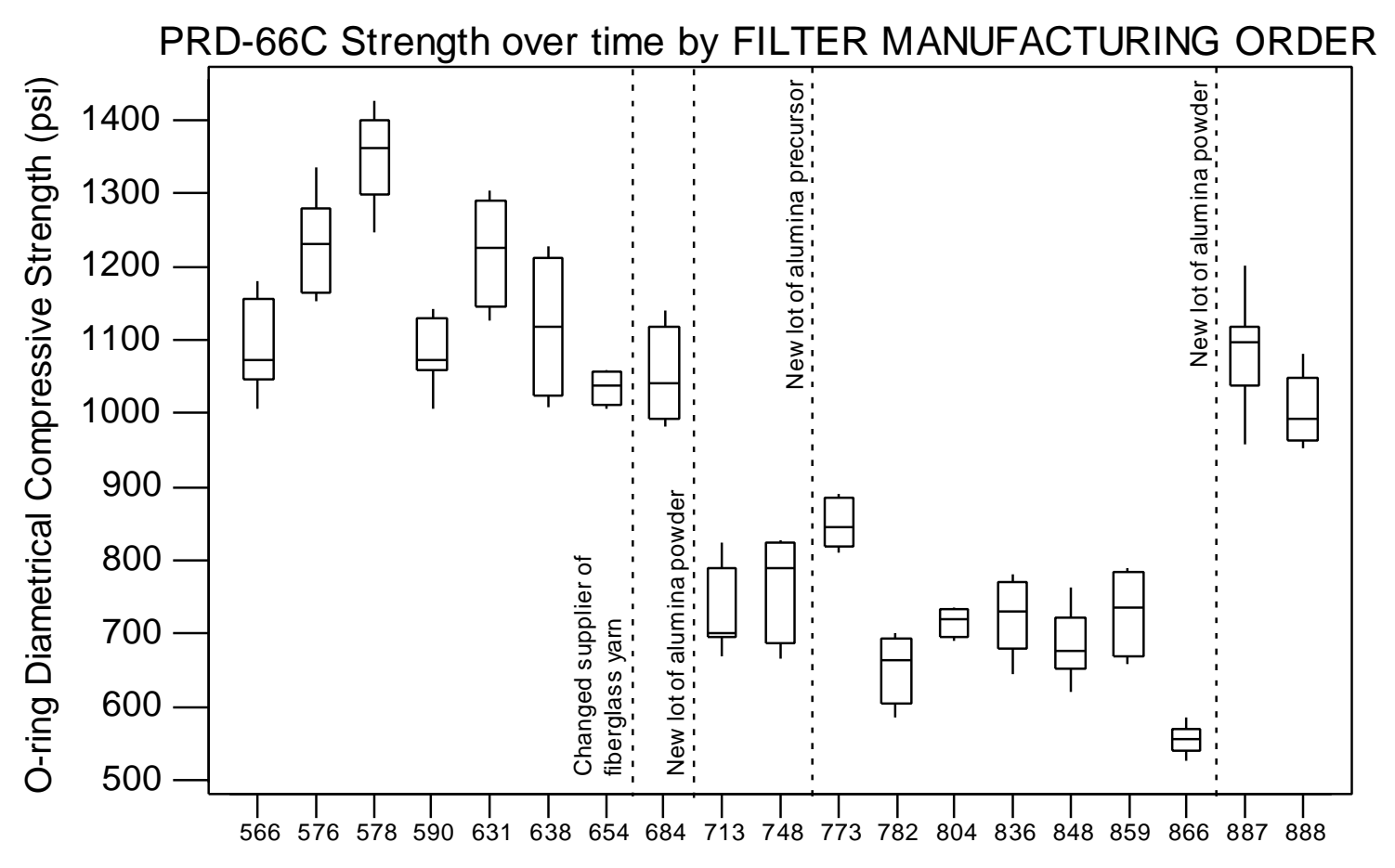

Filter Identification Number

Figure 85 - Box Plot of o-ring compressive strength for as-manufactured filters. (each box represents all o-rings from a particular filter)

In general, filters are fired in roughly the same order in which they are fabricated on the winding machines. It was important, therefore, to determine if a shift in the furnace conditions 
might have coincided with the change to the new lot of alumina powder. Historically, the thermal history the PRD-66 material has a significant impact on the strength of the material. At approximately the same time that the "questionable lot" of PSDF filters was fabricated, two changes were made to the furnace that could possibly have had an effect. These events are indicated on the graph in Figure 86.

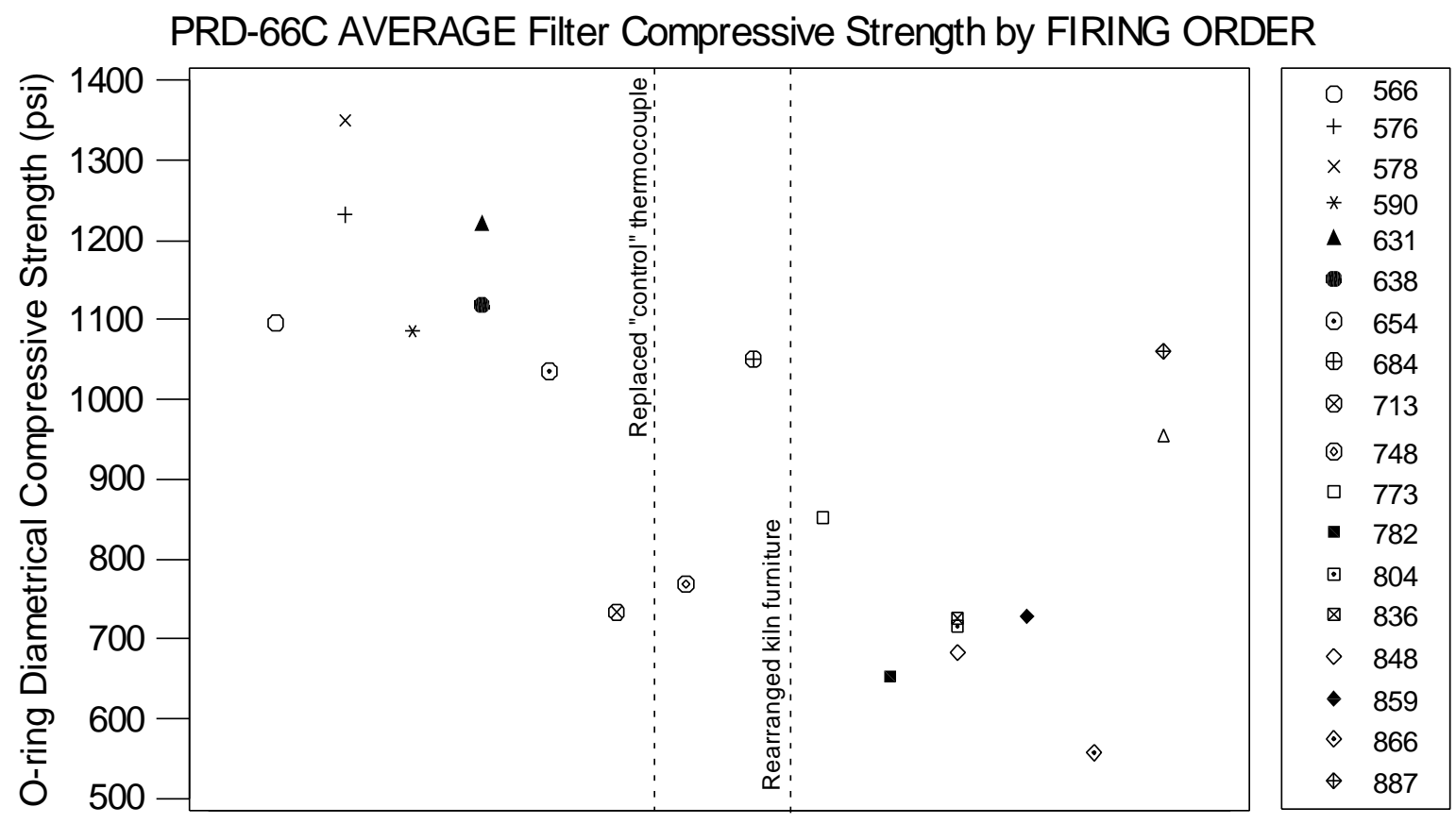

Firing Order

Figure 86 - Average o-ring compressive strength for as-manufactured filters, plotted in the order in which they were fired.

The most significant feature is that filter \#684 was fired during the same period of time that the "weaker filters" were fired. This filter, in fact, had been chosen as the "representative" filter for destructive testing, as required by the Westinghouse protocol, because it was "nominal" in all observable characteristics except that it had a short flange (out-of-spec). The fact that filter \#684 was similar in strength to other filters made with the first lot of alumina powder reinforces the previous observation that the second lot of alumina contributed to the lower strength. Figure 87 displays how distinctly different the filter populations were depending on which alumina lot was used. 


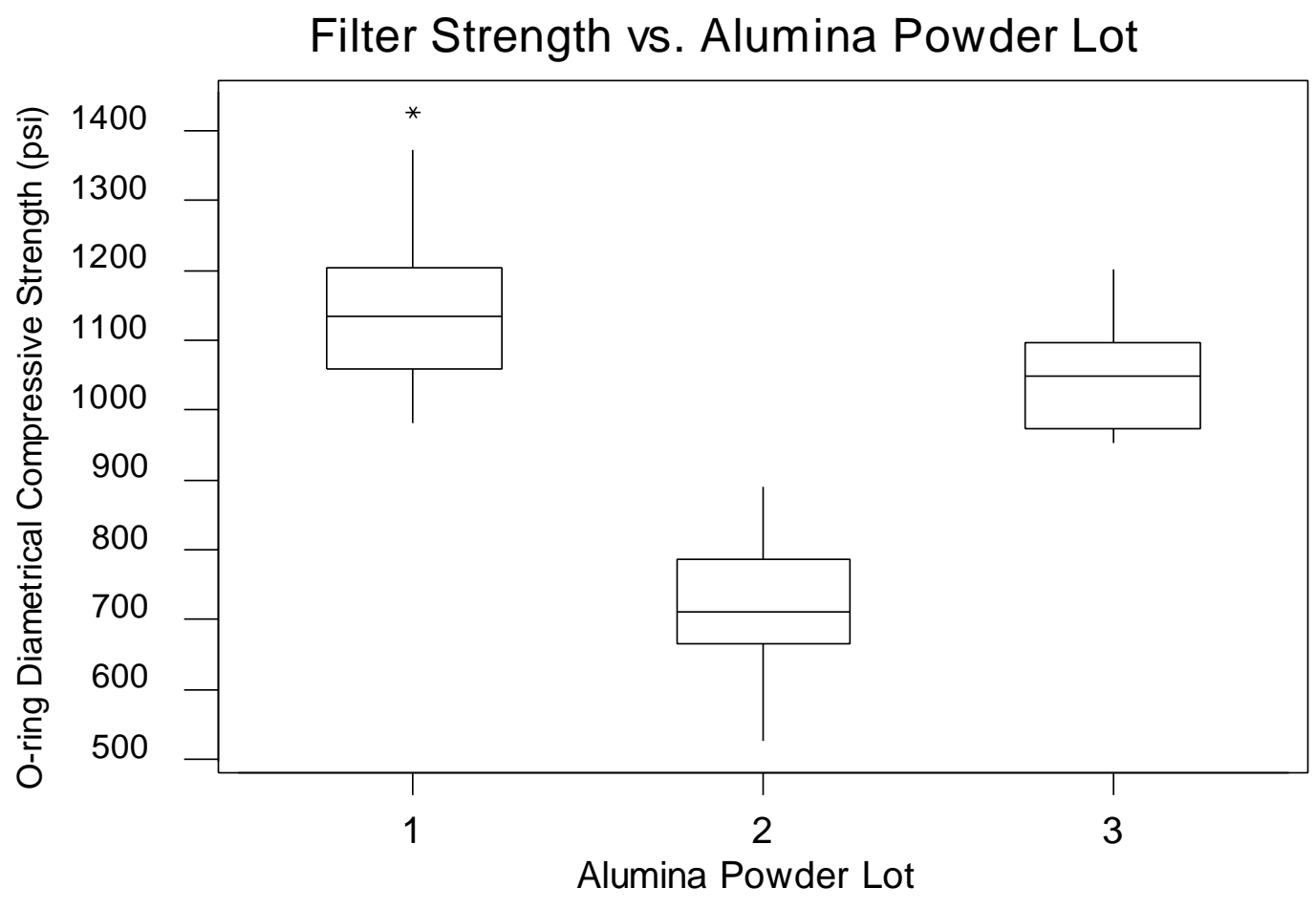

Figure 87 - Box Plot of o-ring compressive data grouped by alumina lot number.

According to the manufacturer's data on the "good" and "questionable" lots of alumina, all the material was within specification for impurities and particle size distribution. There was, however, as slight difference in the median particle size, which indicated that the batch with the slightly finer powder yielded a higher strength product. It would have been beneficial to conduct a more detailed particle sized distribution analysis of the powders, but Alcoa had no retained samples, and none were kept by AlliedSignal. Analysis was conducted, however, on the filters made by the different lots of alumina. Samples of filters made with Alumina Lot\#1 and Alumina Lot\#2 were tested by energy dispersive $\mathrm{x}$-ray spectroscopy. The semi-quantitative results indicated the presence of magnesium, aluminum, and silicon - the three constituents of PRD-66 raw materials. With regard to two possible problem contaminants (sodium and calcium), none were detected.

Historically, the strength of PRD-66 products can be impacted by the crystalline phases present and their relative proportions. The three primary phases are corundum (alumina), mullite (aluminum-silicate), indialite (magnesium-aluminum-silicate), and less than 5\% cristobalite (silica). Strength decreases, which had been observed in the past, resulted from the presence of high levels of cristobalite (caused by an improper firing cycle). Using x-ray diffraction, several crystalline phase comparisons were conducted between filters made at different times with 
different batches of alumina. A comparison of the "high strength" C638 and "low strength" C713 is shown in Figure 88. There were no unusual crystalline phases present and peak heights of the existing phases were similar. A more thorough quantitative phase analysis was performed by a different laboratory on a couple of the most critical samples. A nominal composition is shown in Table 22. Within the accuracy of the analysis (+/- 5\%), there was no significant difference between the samples, except for a slightly lower amount of corundum (alumina) in filters that were known to have a lower alumina content. Significantly larger numbers of samples would be required to detect subtle differences. It can be concluded, therefore, that the lower strength of the “Alumina Lot \#2 filters” was not caused by a different crystalline phase mixture.

Table 22 - Nominal Crystalline Phase Composition of PRD-66

\begin{tabular}{|c|c|c|c|c|}
\hline $\begin{array}{c}\text { Cordierite } \\
\text { (Indialite) }\end{array}$ & $\begin{array}{c}\text { Corundum } \\
\text { (Alumina) }\end{array}$ & Mullite & Cristobalite & Amorphous \\
\hline $35 \%$ & $30 \%$ & $20 \%$ & $5 \%$ & $10 \%$ \\
\hline
\end{tabular}

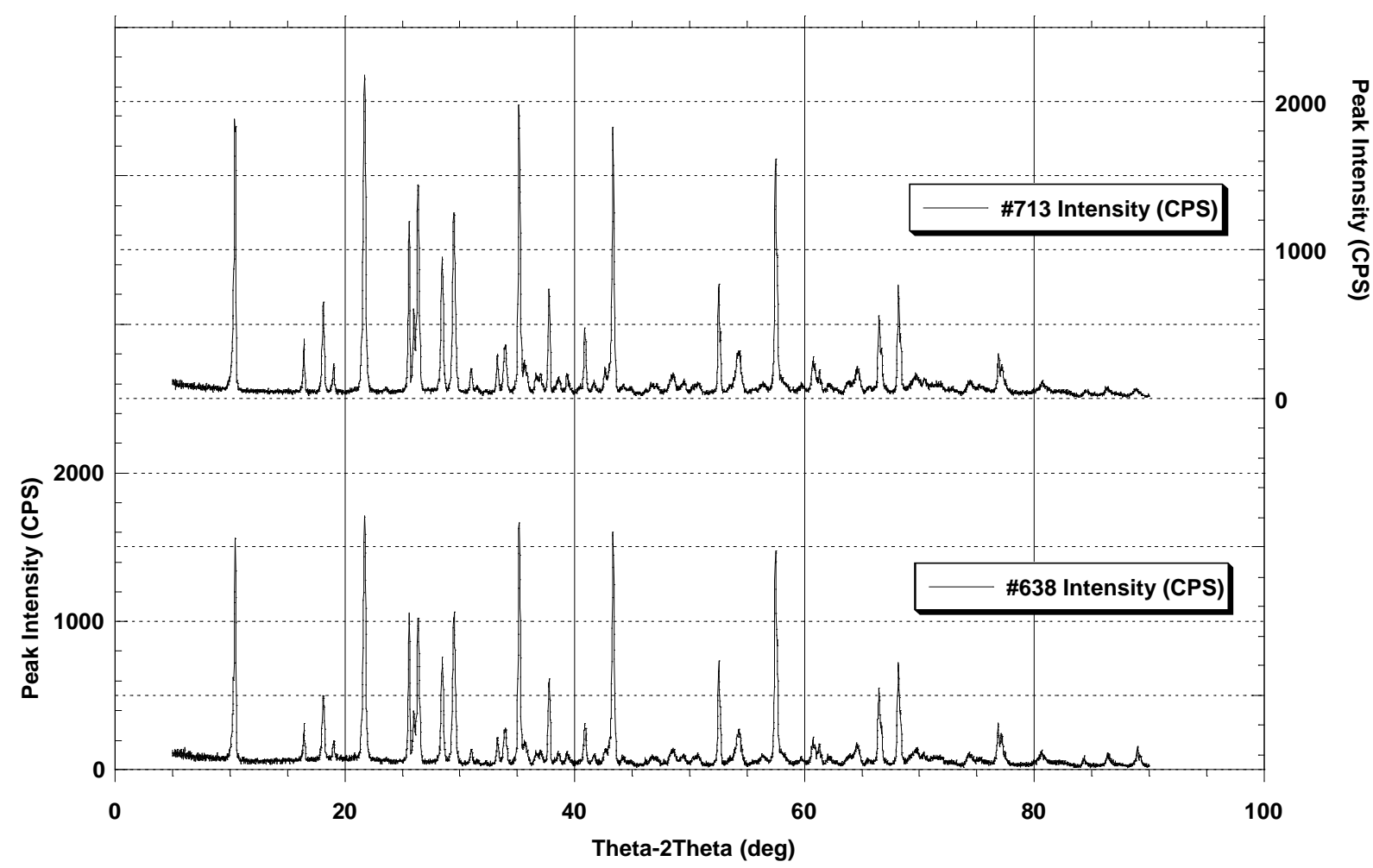

Figure 88 - X-ray Diffraction Comparison of Filter \#638 (Alumina Lot \#1) and Filter \#713 (Alumina Lot \#2) shows no differences in crystalline phases. 
In a attempt to corroborate the potential link between "alumina lot" and strength, additional sampling was conducted. O-rings were cut from three as-manufactured filters, which had been shipped to the PSDF. The data was compared to previous information collected on filters made with the three lots of alumina powder. All three PSDF filters had been made with "Lot \#2" alumina; their strengths are compared to previous data in Table 23. The strength of filters \#C739 and C\#751, is most similar to other "Lot\#2" filters, as expected. Filter \#C733, however, is distinctly higher than the other PSDF filters and the other "Lot \#2" filters.

Table 23 - O-ring Strength of As-Manufactured PRD-66 Filter $(\mathrm{N}=$ number of specimens tested)

\begin{tabular}{|c|c|c|c|}
\hline & & \multicolumn{2}{|c|}{ O-ring Diametrical Compressive Str. (psi) } \\
\hline Alumina Lot & $\mathbf{N}$ & Average & Std. Dev. \\
\hline Lot \#1 & 54 & 1146 & 110 \\
\hline Lot \#2 & 68 & 713 & 91 \\
\hline Lot \#3 & 20 & 1044 & 70 \\
\hline & & & Std. Dev. \\
\hline Lot \#2 Filters from PSDF & $\mathbf{N}$ & Average & 103 \\
\hline C733 & 9 & 1022 & 41 \\
\hline C739 & 9 & 815 & 69 \\
\hline C751 & 9 & 855 & \\
\hline
\end{tabular}

A close examination of the production records for this filter indicated that it was manufactured on a day when the relative humidity was at $85 \%$. Standard practice is to control the humidity in the air by "adding water" and maintaining 50-65\% RH. On the day that filter C733 was fabricated, however, the water addition system was inactive because the ambient relative humidity was already above the upper limit.

Historically, fabrication of filters with less than $30 \%$ RH can cause adhesion problem between adjacent layers of slurry-coated yarn. This data suggests that elevated humidity could lessen the impact of the alumina powder; it also suggests that improved o-ring compressive strength might be achieved by fabricating the filters under more humid conditions. 
In addition to o-ring diametrical compression testing, studies were also conducted on the axial tensile properties of the material by cutting 8 " long coupons from the wall of the filters as shown in Figure 89 and Figure 90. The tensile strength values were highly variable and did not correlate well to the o-ring data, however, the shape of the "stress versus strain curves" revealed some interesting data.

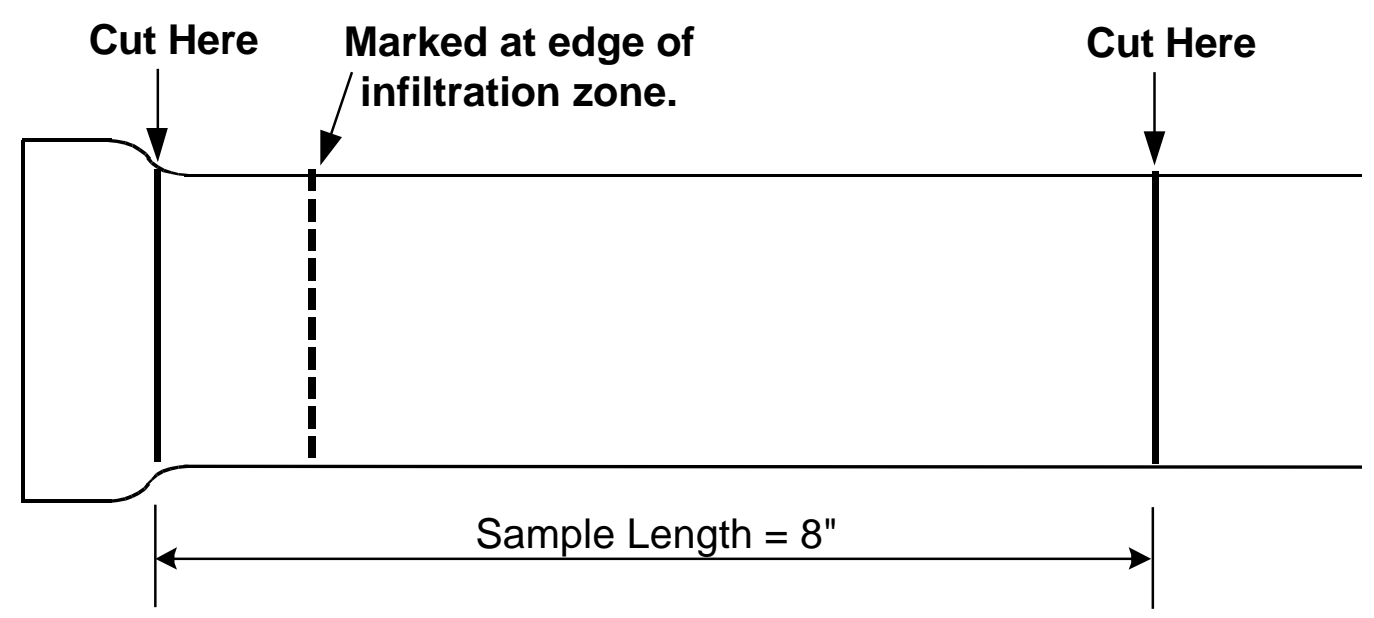

Figure 89 - Cutting plan for tensile coupons from PRD-66C "as-manufactured filters".

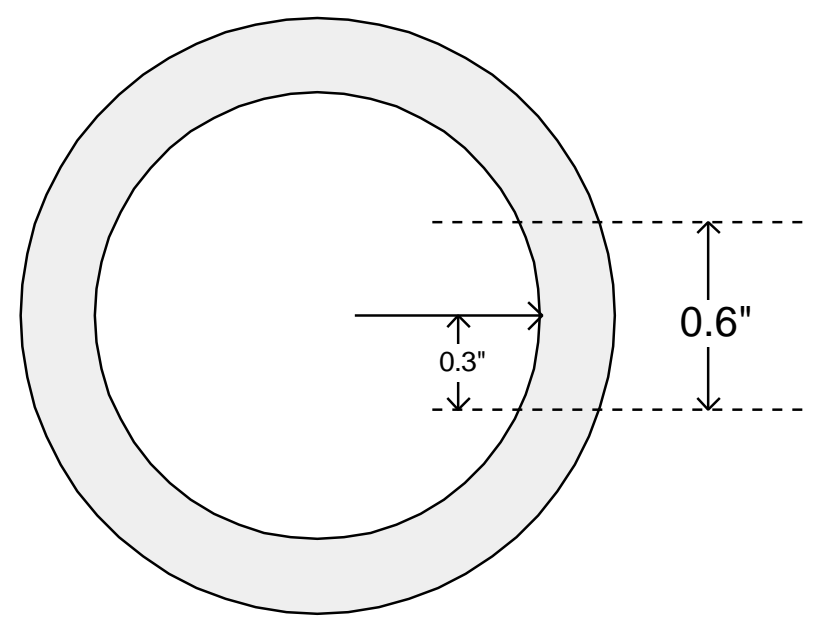

Figure 90 - Filter cross-section depicting geometry of tensile coupons.

Figure 91 shows the stress/strain curves for two filters made with Alumina Lot\#1. The coupons had similar breaking strength, as shown on the "Stress" axis; in addition, both materials absorbed the stress in a similar manner, as depicted by the curved shape of the plot and the maximum strain. The area under the stress/strain curve, is often considered to be an indicator of "toughness". The calculated "area under the curve" is displayed on each plot. 
The stress/strain curves of the Alumina Lot\#1 filters were compared to the Alumina Lot\#2 filters, which are shown in Figure 92. Although these materials withstood a similar stress before breaking, they absorbed the load in very different ways as indicated by the straightness of the line. This difference can be quantified by comparing the "area under the curve", which suggests that the filters made with Alumina Lot\#2 may not be as "tough" as those made with Alumina Lot\#1.
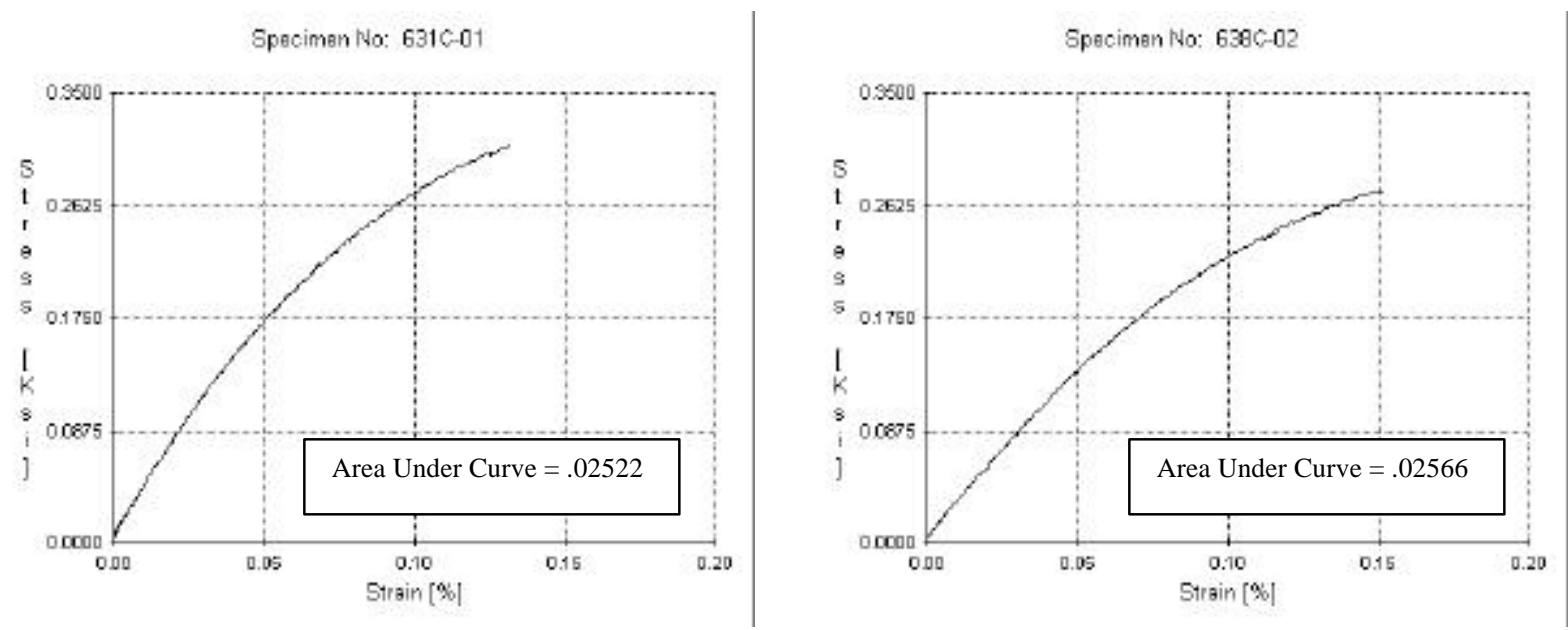

Figure 91 - Axial Tension stress/strain curves for unexposed filters made with $\underline{\text { Alumina Lot\#1 }}$
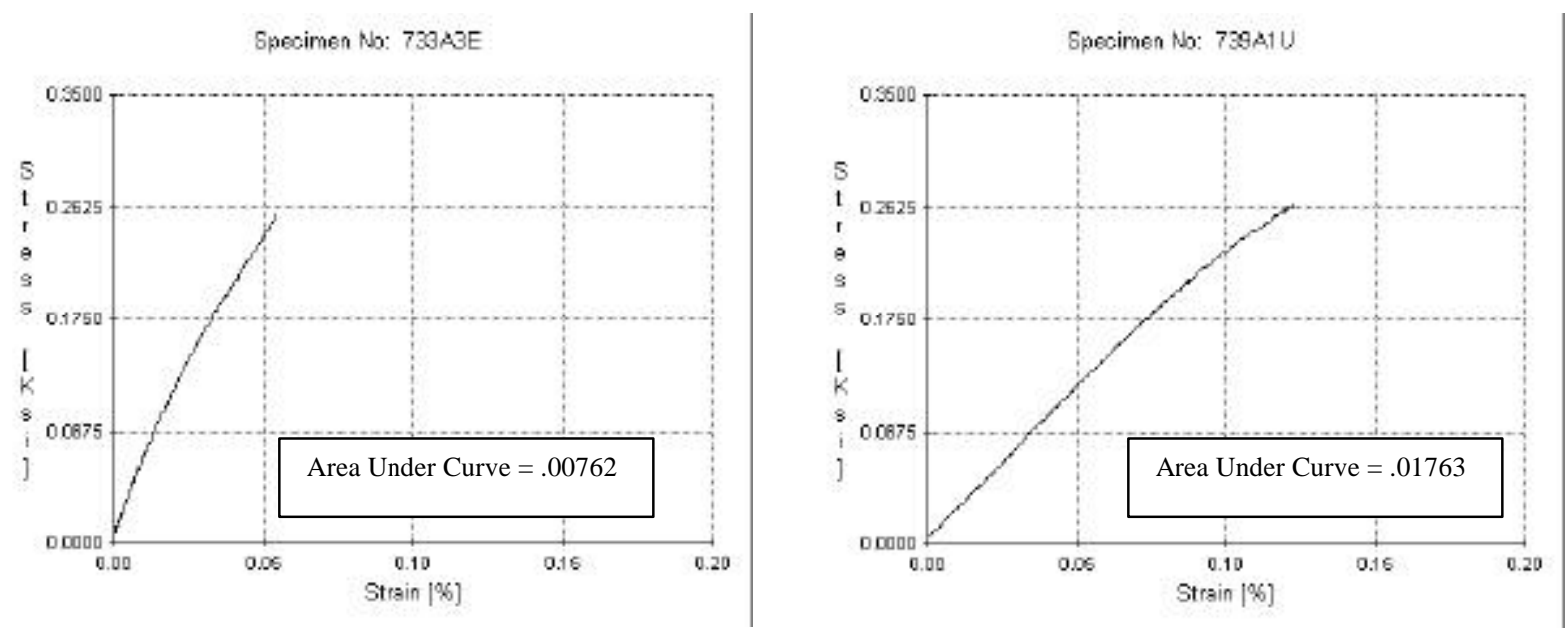

Figure 92 - Axial Tension stress/strain curves for unexposed filters made with Alumina Lot\#2

Although evidence was found to link the low strength filters to "Alumina Lot \#2", it did not explain the reason for it. An extensive study of the microstructure was undertaken to determine if a visual difference existed. Figure 93 is a polished cross-section of as-manufactured filter \#C567, which had been made for the Westinghouse qualification tests conducted in Karhula, Finland. The specimen was examined with an optical microscope.

In examining Figure 93, the following explanation describes how the microstructure relates to the make-up of the material: 
During firing, the fiberglass yarns devitrified (crystallized) and the filaments, which made up those yarns, can be seen as the round, darker areas in the micrograph. The alumina matrix appears as the lighter-colored elongated particles. The alumina particles are primarily located on the exterior of the yarns, but a significant amount does penetrate the yarn bundle. Another common feature in the microstructure of \#C567 (and other filters made with "alumina lot \#1") was the presence of many small voids on the surface of the polished sample, which appear as the darkest spots on the micrograph. It is unknown whether these voids represent the inherent porosity of the material or if they are polishing artifacts, caused by the break-out of particles during the polishing process. Filter \#C567 had an average o-ring diametrical compressive strength of 1241 psi.

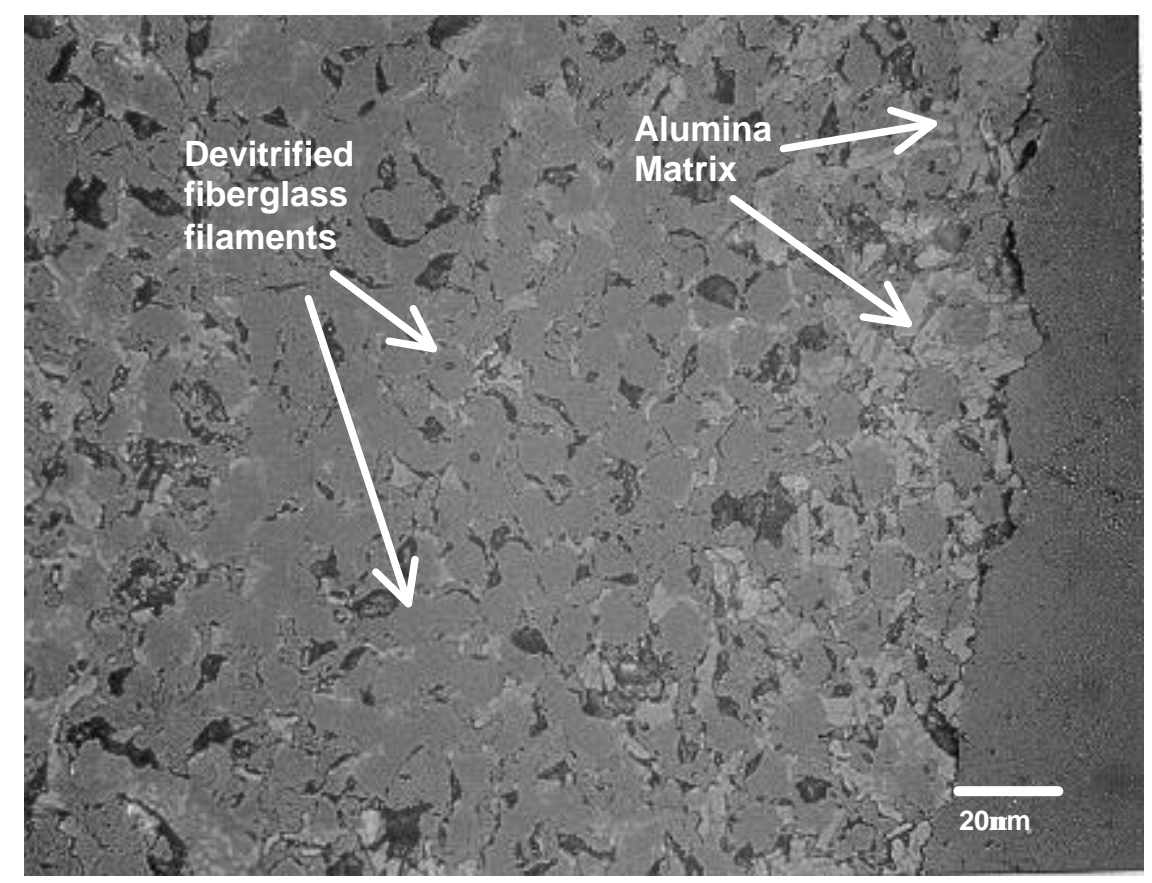

Figure 93 - Microstructure of PRD-66C Filter \#C567, 500X

Figure 94 shows as-manufactured filter \#C713, made with "alumina lot \#2", having an average o-ring diametrical compressive strength of 785 psi. This sample has many of the same features as \#C567, such as similar sized alumina grains and some fusion of adjacent filaments. This particular region of $\mathrm{C} \# 713$ also shows that areas where two yarns cross have larger regions of 
alumina matrix in between them; \#C567 had similar areas. In general, there were no major differences in the microstructure, between filters made with "alumina lot \#1" and "alumina lot \#2", except for the presence of the voids noted in the \#C567. All "alumina lot \#2" filters had fewer, smaller voids.

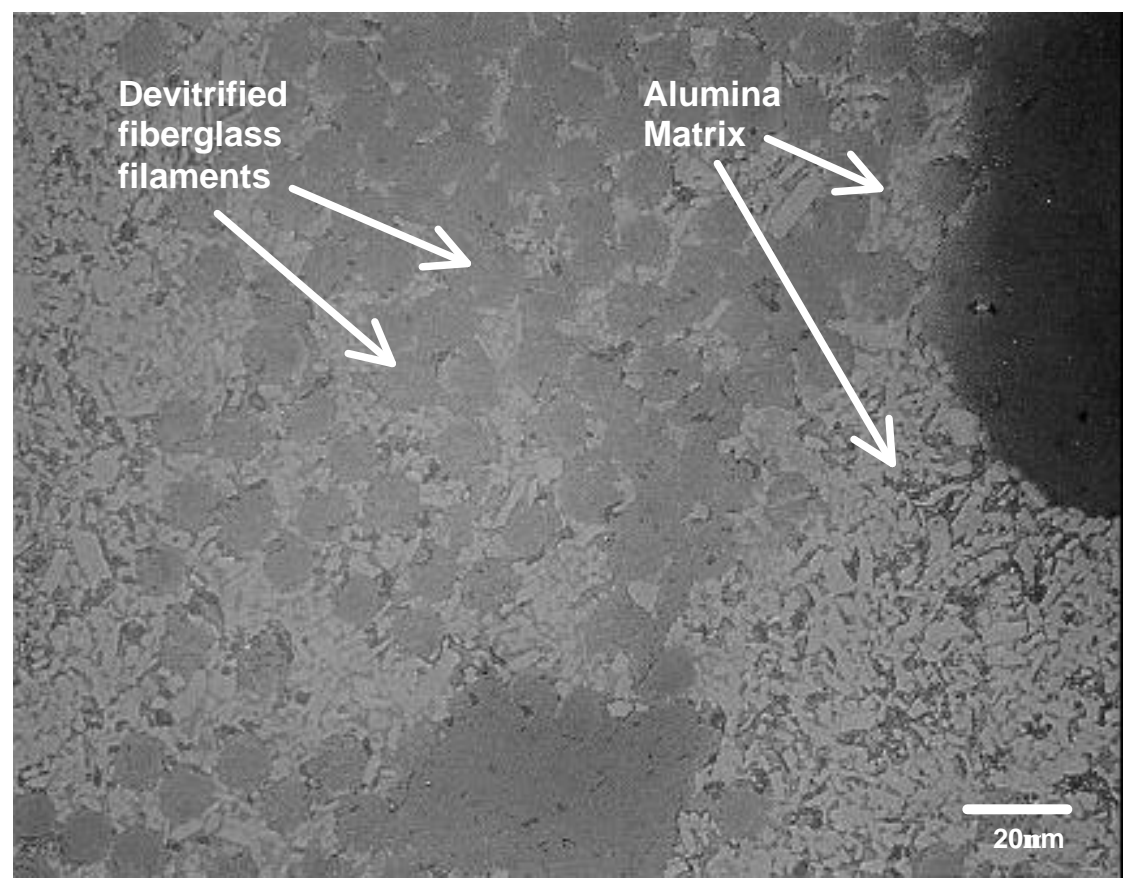

Figure 94 - Microstructure of PRD-66C Filter \#713, 500X

A closer examination was conducted on the microstructure of Filter C752, which broke at the base of the filter nut, as it was being removed from the plenum. This was the only filter that broke below the flange, for which we have possession of the broken filter, the other three filters, which broke in a similar manner, fell into the ash hopper. Examination of this filter, shows that its microstructure (Figure 95) is similar to other filters made with the same raw materials, such as filter \#C713. It is significant to note that its microstructure, as well as those of other "exposed filters" shows that no apparent change results from the exposure of the filter to PFBC conditions. 


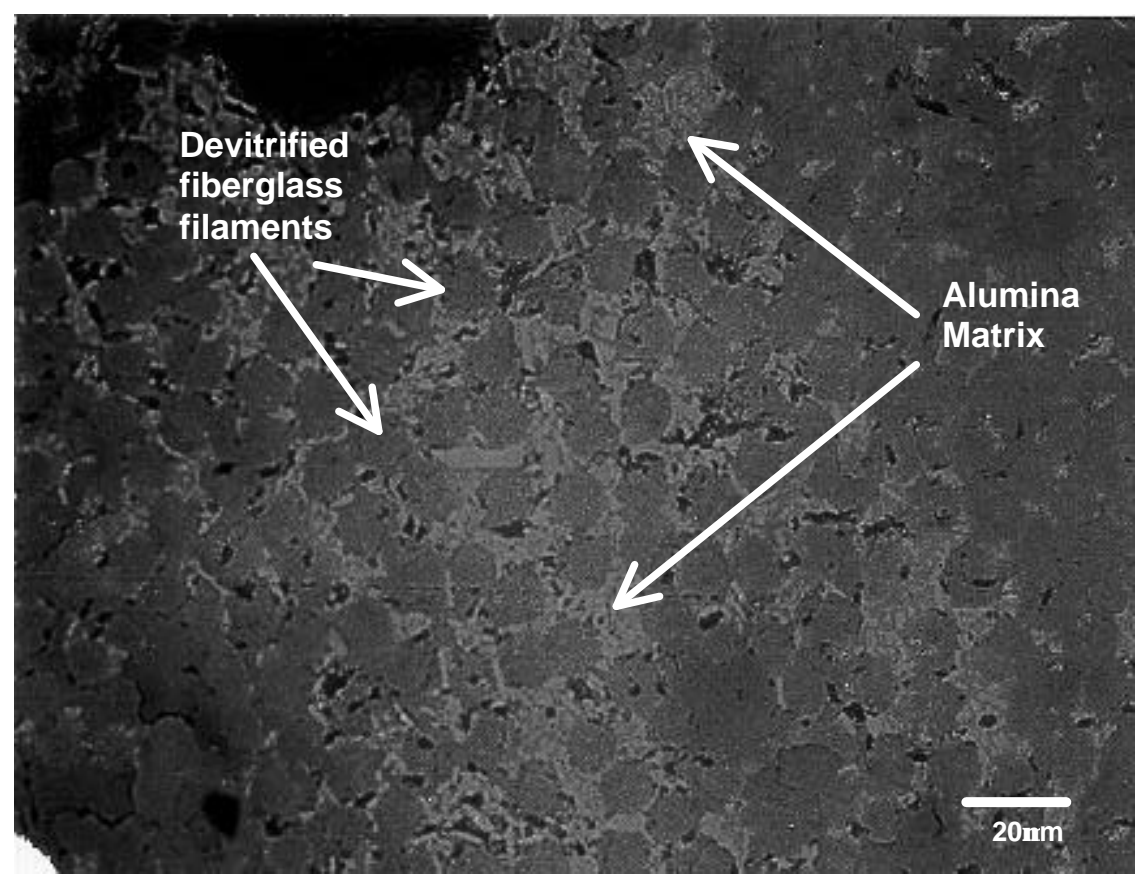

Figure 95 - Microstructure of PRD-66C Filter \#C752, 500X

In general, the polished microstructures were very similar except for the presence of more apparent "voids" in the stronger filters made with "alumina lot \#1".

Additional examinations were conducted on "fresh-fracture surfaces", to determine if the "voids" in the polished specimens were really pores in the microstructure or if they were caused by the "break-out" of particles during the polishing process. The fresh-fracture specimens were from high and low strength filters and were examined using a scanning electron microscope (SEM).

Both the high and low strength materials exhibited similar inhomogeneities within their structures, as shown in Figure 96 and Figure 97. In regions where the alumina particles, which comprise the matrix, surrounded the devitrified fiberglass filaments, the filaments remained round with minimal fusion of the filaments to each other or to the matrix, as shown in Figure 98. In regions where the very little alumina was in contact with the filaments (usually in the center of a bundle of filaments), adjacent filaments tend to fuse together, as shown in Figure 99. 


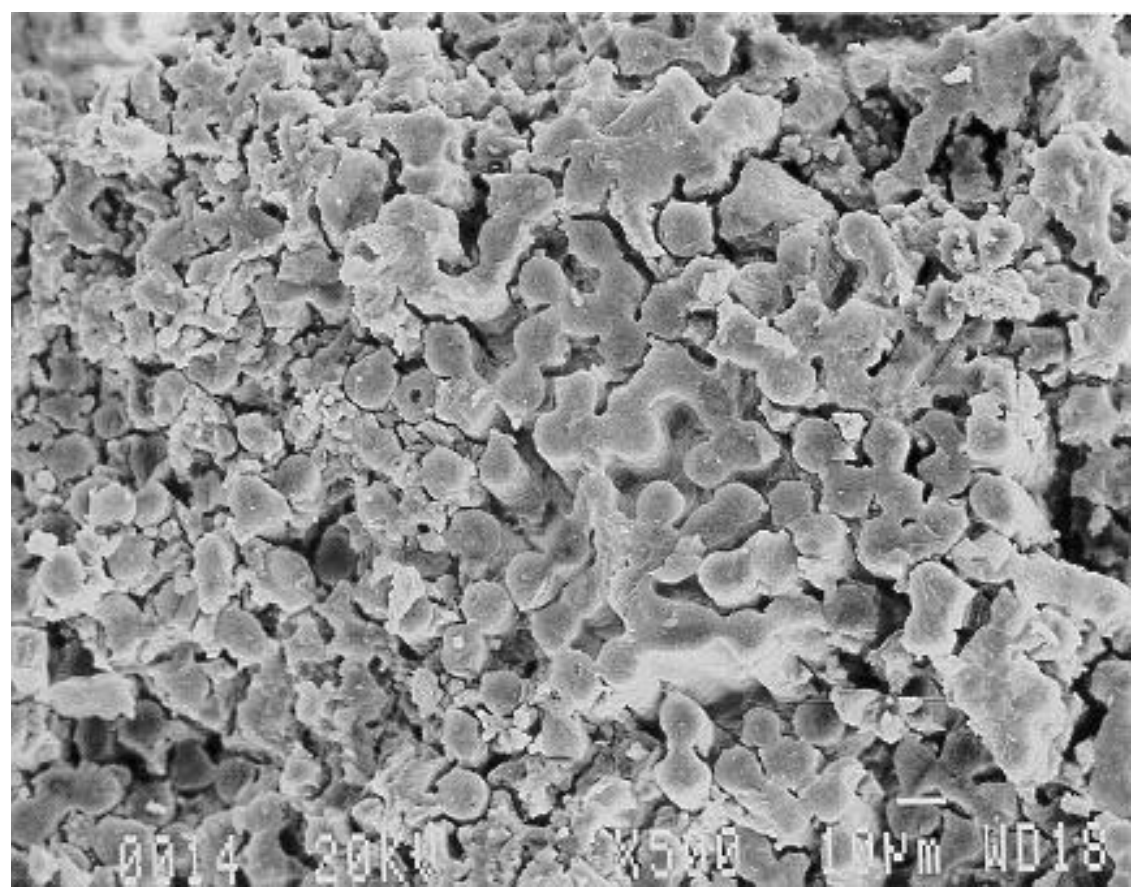

Figure 96 - Fresh-fracture SEM of PRD-66C Filter \#C567 (high strength) at 500X

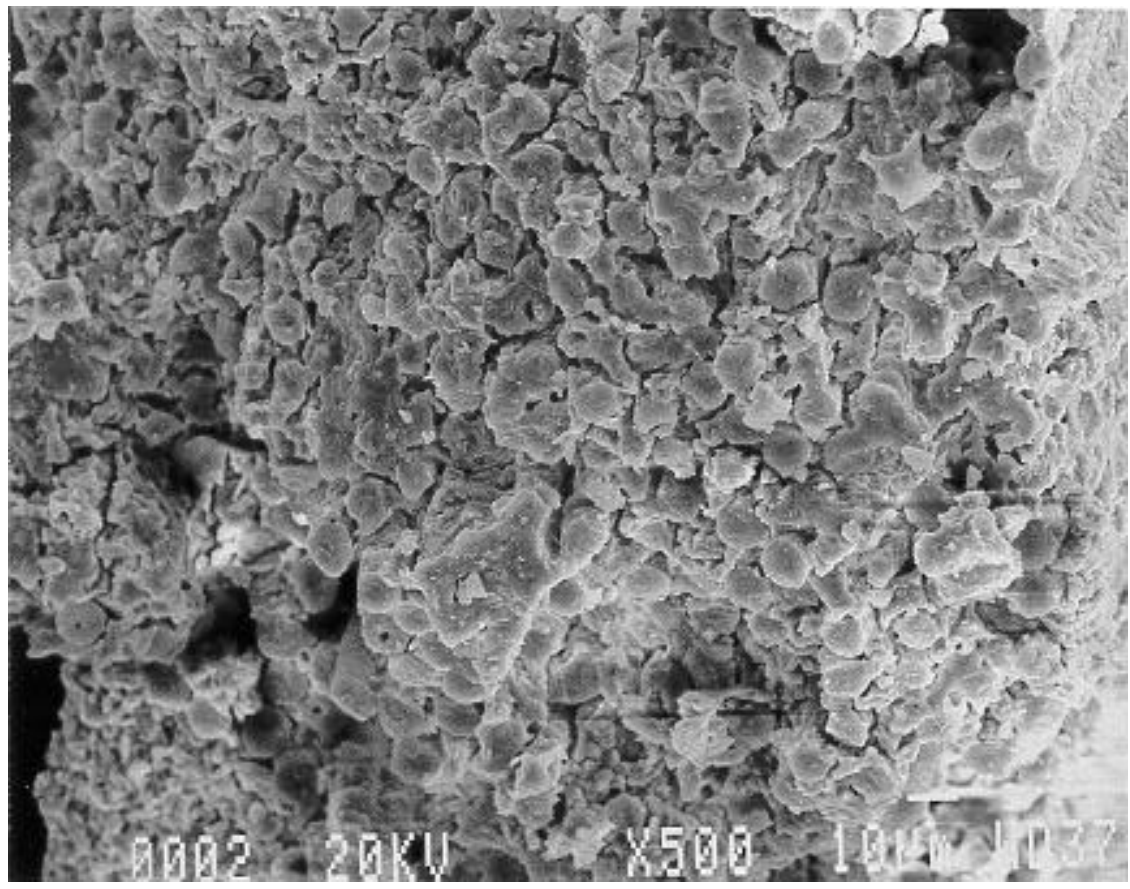

Figure 97 - Fresh-fracture SEM of PRD-66C Filter \#C713 (low strength) at 500X 


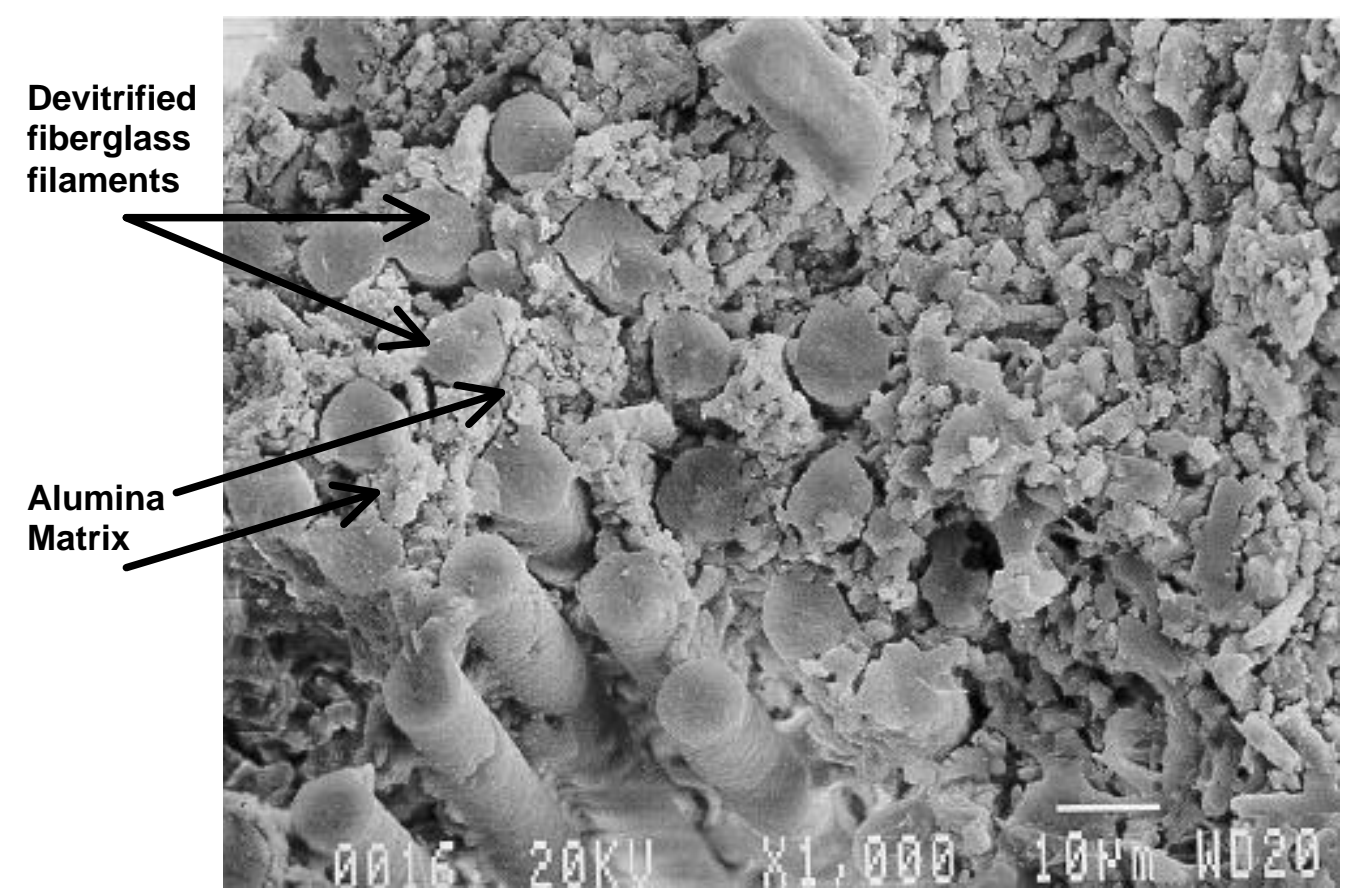

Figure 98 - Fresh-fracture SEM of \#C567 in high-alumina region at 1,000X

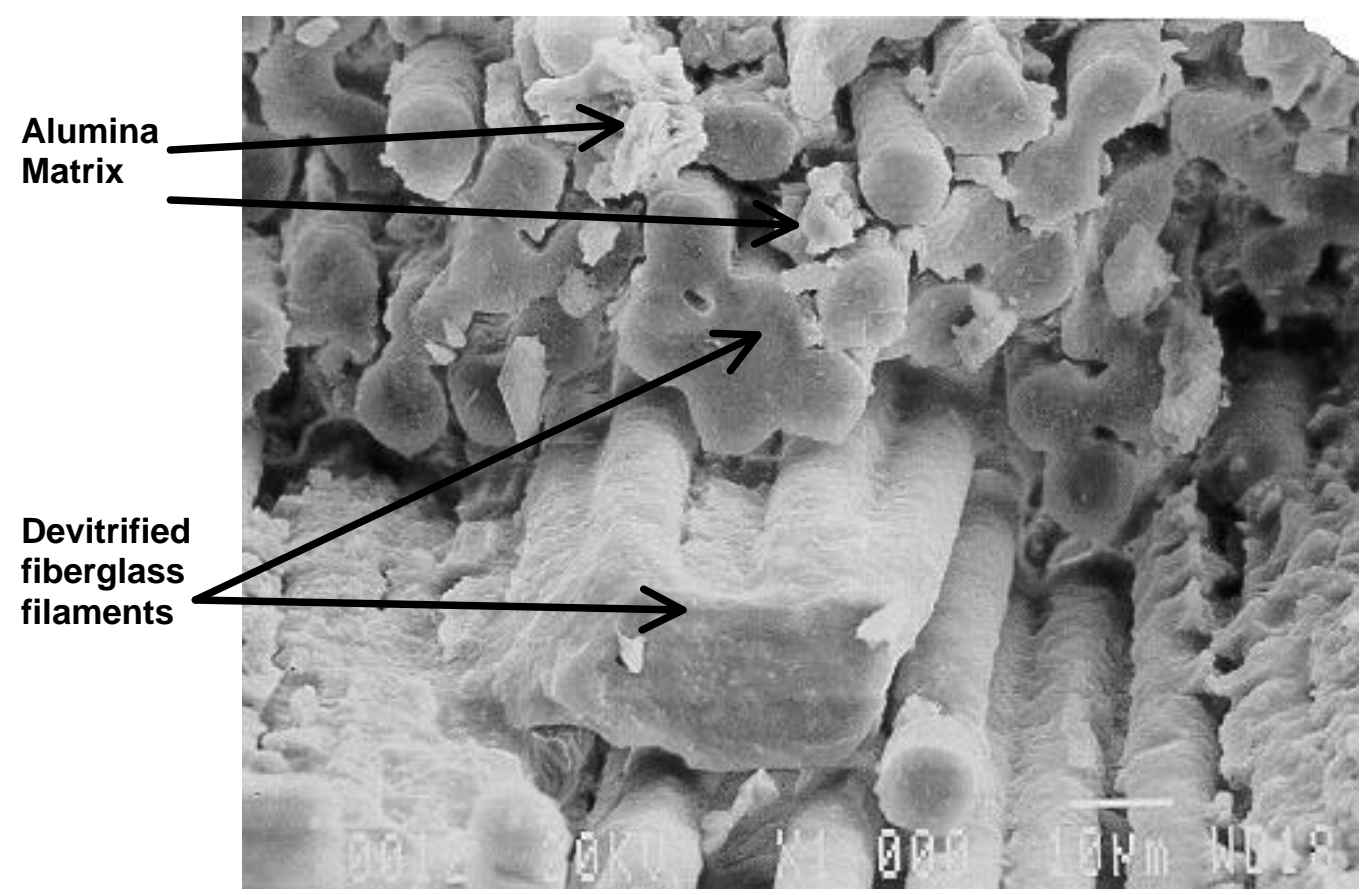

Figure 99 - Fresh-fracture SEM of \#C567 in low-alumina region at 1,000X 
Although this examination showed no apparent difference between the high-strength and low-strength filters, it did exemplify the variety of microstructures that result from the presence of both high and low alumina regions, which are inherent in the manufacturing process. In addition, the SEM examinations suggested that all microstructures had similar levels of porosity.

Besides the raw materials used in the production of the filters, another concern was the amount of alumina within the final product. The presence of alumina, around and within the yarn bundles, can dramatically effect the microstructure, as documented with the use of optical and scanning electron microscopes. For filters made with Alumina Lot \#2, all o-ring diametrical compression data was collected and plotted as a function of "\% Alumina". The "\% Alumina" was defined as the additional weight percent of alumina particulate added during the winding process. It was calculated by comparing the weight of the wound filter to the amount of fiberglass yarn used to fabricate it. The average strength was plotted in Figure 100, but no apparent correlation with alumina content existed.

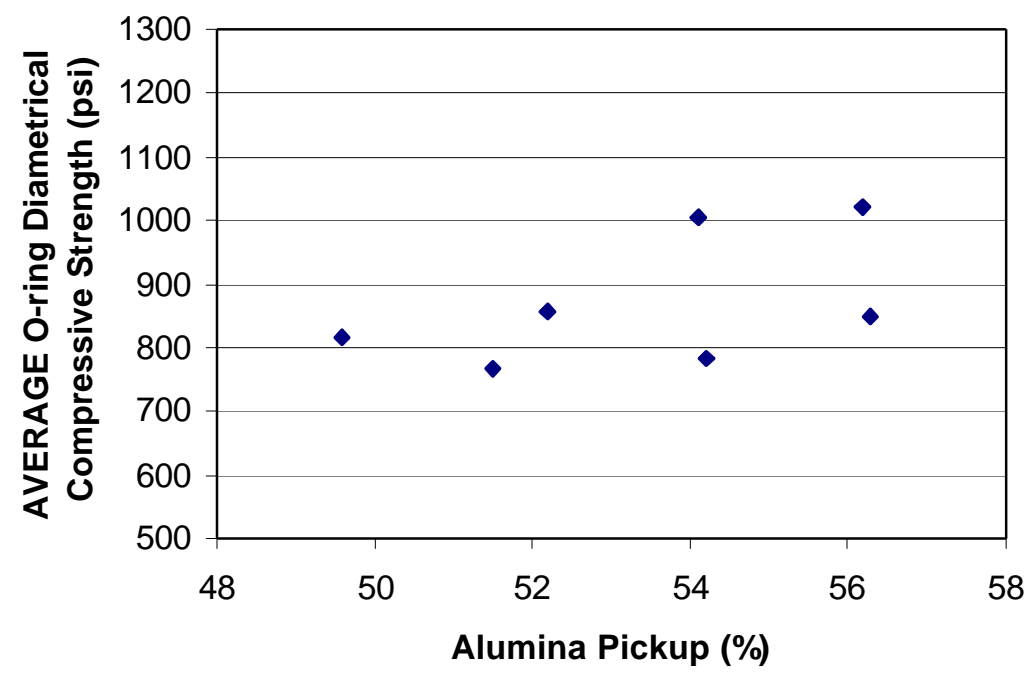

Figure 100 - Alumina content versus average o-ring diametrical compressive strength for as-manufactured PRD-66 filters made with Alumina Lot \#2

A similar analysis was performed on the axial tensile data collected on several of these filters, but no correlation was found. The fact that all of the broken filters had a low alumina content, therefore, could not be sited as the reason for their failure. 
Another feature that all of the broken filters had in this study was the fact that they fractured in a location that corresponded to "the edge of the infiltration zone" and "the base of the filter-nut supported the element". (The development of this "strengthened flange" was described in Section 3.2.4, Page 15). This coincidence raised concerns that the point where the infiltration ends could be more fragile then the area to either side, or if the zone was too short it would not provide adequate support.

Since some of the axial tensile coupons used in the study described in Figure 89 (Page 102) contained the infiltrated region of the flange, an analysis of the breaking characteristics was conducted. It was noted that all of the coupons, which had at least nominal alumina content, broke near the center. A couple of the "low alumina" specimens, however, broke at the edge of the infiltrated zone. There were too few data points to draw any supportable conclusions, but additional investigations were warranted, particularly in regard to how this region of the filter behaves under flexural loads. Flexural loads could become a significant factor when the filter vibrates in-service. The staff of the PSDF had long been concerned about the effect of vibration on the filters, particularly the PRD-66C and McDermott filters that visibly "wiggled" while raising and lowering the plenum ${ }^{13}$. It was beyond the scope of this study to analyze vibration characteristics, however, ways to measure flexural strength of the PRD-66 filter were investigated. Initially, a "Cantilever Flange Bend Test", developed at Virginia Tech ${ }^{16}$, was considered, but only one test could have been performed on each filter. Unfortunately, there were not enough unexposed candles from the "problem lot" of filters to obtain meaningful results.

Another test method considered was a "4-point bend" technique, which could be conducted on several coupons from a single flange. In order to determine how the "infiltrated", "uninfiltrated" and "transition" zones responded to flexural stresses, one filter was tested to determine if it gave meaningful results. Coupons were prepared by slicing 3" long X 0.6" wide bars from a filter, as shown in Figure 101 and Figure 102. The coupons were taken from various regions, such that the test fixtures would apply force in one of three locations: in the infiltrated zone, in the uninfiltrated zone, in the transition zone. 


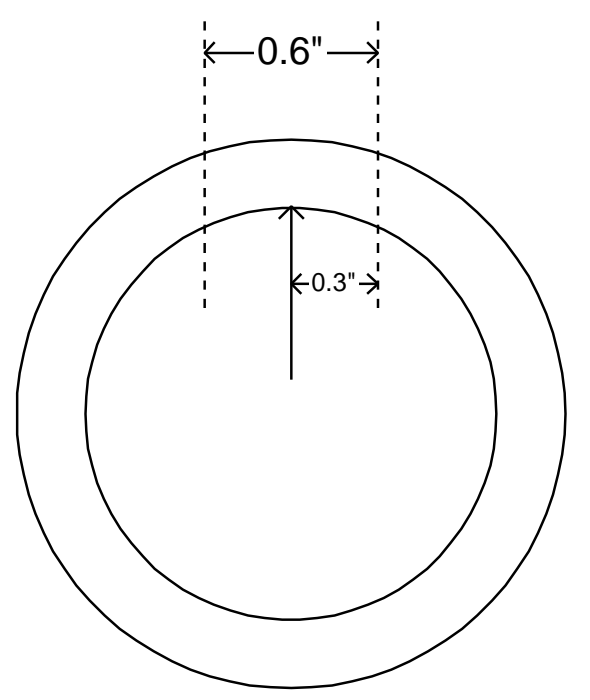

Figure 101 - PRD-66 4-point flex coupons - cross-section

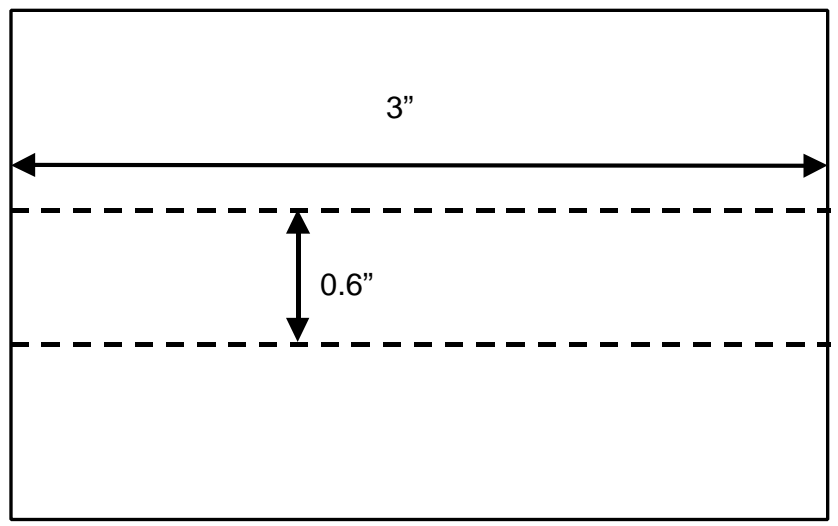

Figure 102 - PRD-66 4-point flex coupons - side-cut view

In the test, the membrane side of the coupon was positioned "down" with the span of the lower supports equal to $40 \mathrm{~mm}$; the pressure was applied to the inside diameter surface of the coupon over a $20 \mathrm{~mm}$ span as shown in Figure 103. This test orientation puts the membrane surface in tension.

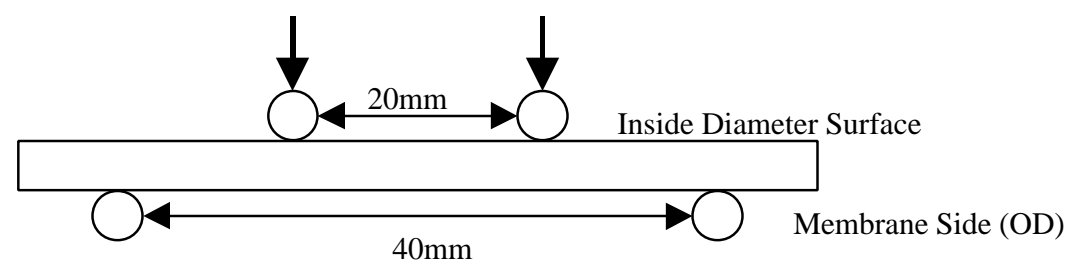

Figure 103 - 4-Point Flex Test set-up of PRD-66 coupons from regions within 4" of the flange

A comparison of the "Maximum Load" in the three types of zones is shown in Figure 104. Although these coupons were all from the same hot gas filter, the variability in the data for each zone was too high to discern any difference between the zones. 


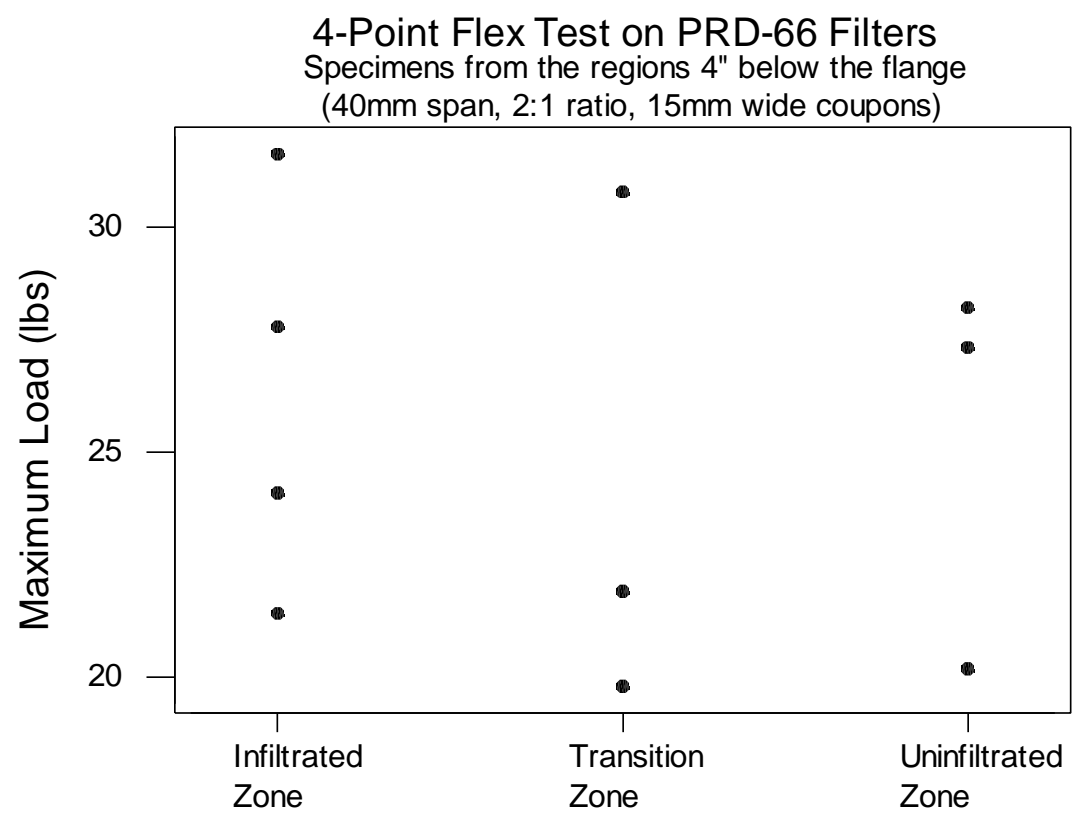

Figure 104 - Maximum 4-Point Load for the Flex Testing of PRD-66 filters in three different regions: infiltrated zone, transition zone, and uninfiltrated zone

The shapes of the "Load versus Displacement" curves were also studied. Within the "infiltrated" and "uninfiltrated" groups of data, there was considerable variability in the peak height and the width of the curve. This suggests that there is too much variability in the material or too much variability in the technique to give consistent results. The shape of the "Load versus Displacement" curves for the "transition (edge) zones" were also very different from each other, as shown in Figure 105. In fact, they have two distinctly different shapes; this may indicate two different types of failure. It is possible that the exact position of the edge of the transition zone within the $20 \mathrm{~mm}$ span is very critical. 


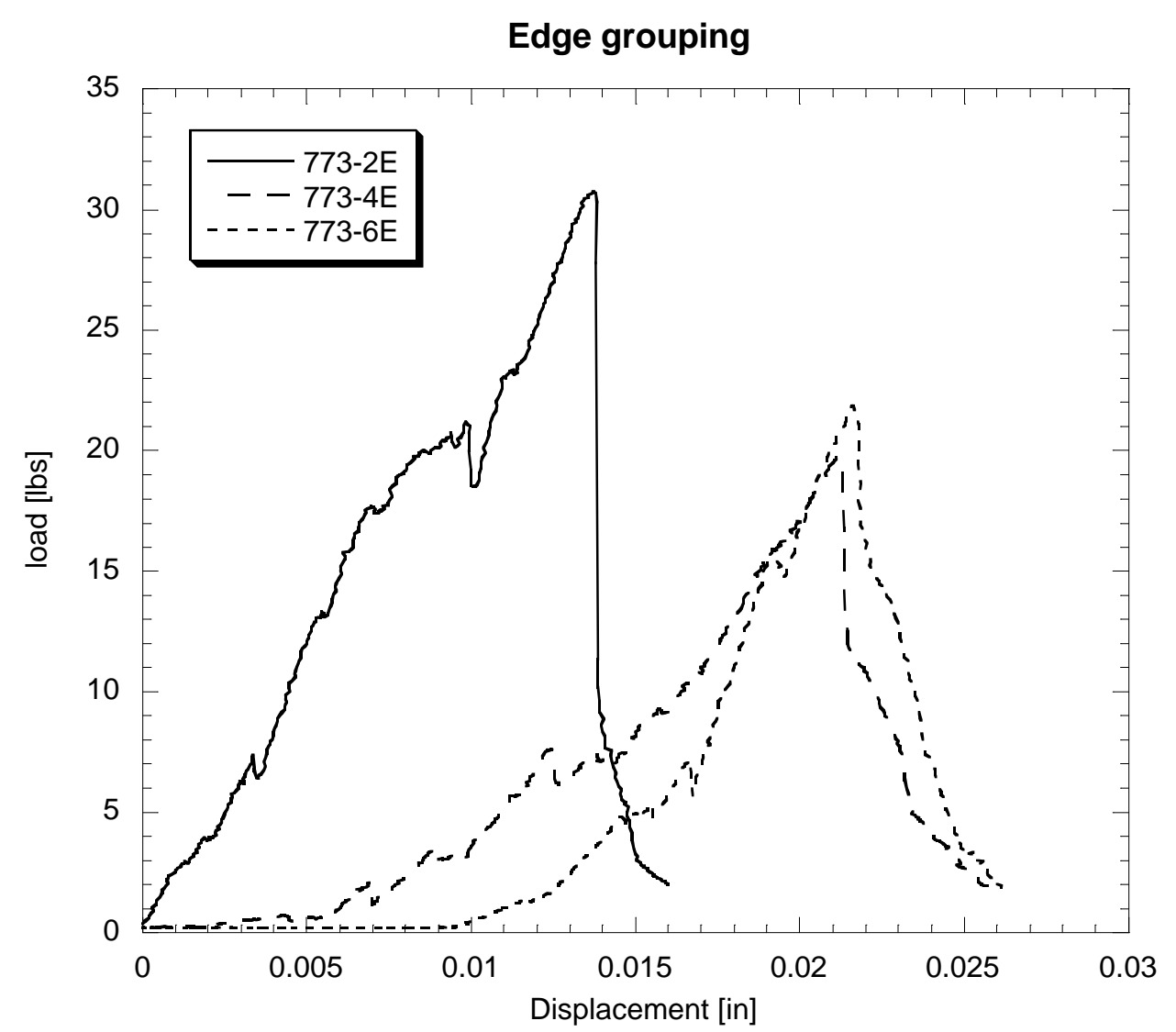

Figure 105 - Load-Displacement Curves for 4-point flex tests in the "transition (edge) zones".

It was hoped that a comparison of the flexural strength of the different zones would indicate which areas were weakest or strongest. The variability in the data, however, made it impossible to draw any meaningful conclusions. A preferred test would actually simulate the fatigue flexure of a cylinder.

This concern over the location of the "edge of the infiltration zone" was brought to the attention of the designers of the Siemens Westinghouse fixtures. Their staff was asked to review the length of the infiltration zone, relative to the location of the base of the filter nut. After their review, a 1" longer infiltration zone was recommended; this change would position the edge of the infiltration zone well below the base of the filter nut. In examining the as-manufactured filters, which were available, it was found that the infiltration zone of the newer filters averaged $3 \mathrm{~mm}$ shorter than the older filters, although they were still within specifications. 


\subsubsection{Non-Destructive Evaluation of PRD-66 Filters}

There are many benefits to being able to test the strength of a candle filter without destroying it. Research programs, being conducted by Roger Chen at the University of West Virginia (UWV) ${ }^{17}$ and Bill Ellingson at Argonne National Lab (ANL) ${ }^{18}$, were primarily focused on evaluating the retained strength of a filter element after exposure. By knowing the retained strength, one can potentially predict how much "life" the filter still has in it and determine whether to reinstall it. It would also be beneficial to use non-destructive methods to assure the quality of as-manufactured filters, particularly if the technique could be used as a predictor of strength.

Roger Chen characterized one PRD-66C filter made with Alumina Lot \#1, but when a filter made with Alumina Lot \#2 was shipped for comparison, it was severely damaged due to mishandling of the shipping crate. Future studies would be useful if more full-length filters were available.

The technique used by Argonne National Lab, however, can be used on segments of filters, which are at least eight inches long. Portions of three as-manufactured PRD-66C filters were evaluated using their acousto-ultrasound (AU) system. Adjacent segments had been subjected to o-ring diametrical compression testing. Figure 106 shows the average "stress wave values" (SWF) for each filter plotted against the average o-ring diametrical compressive strength. Preliminary results appear promising, however, there is considerable spread in the actual data which could not be readily plotted in this figure.

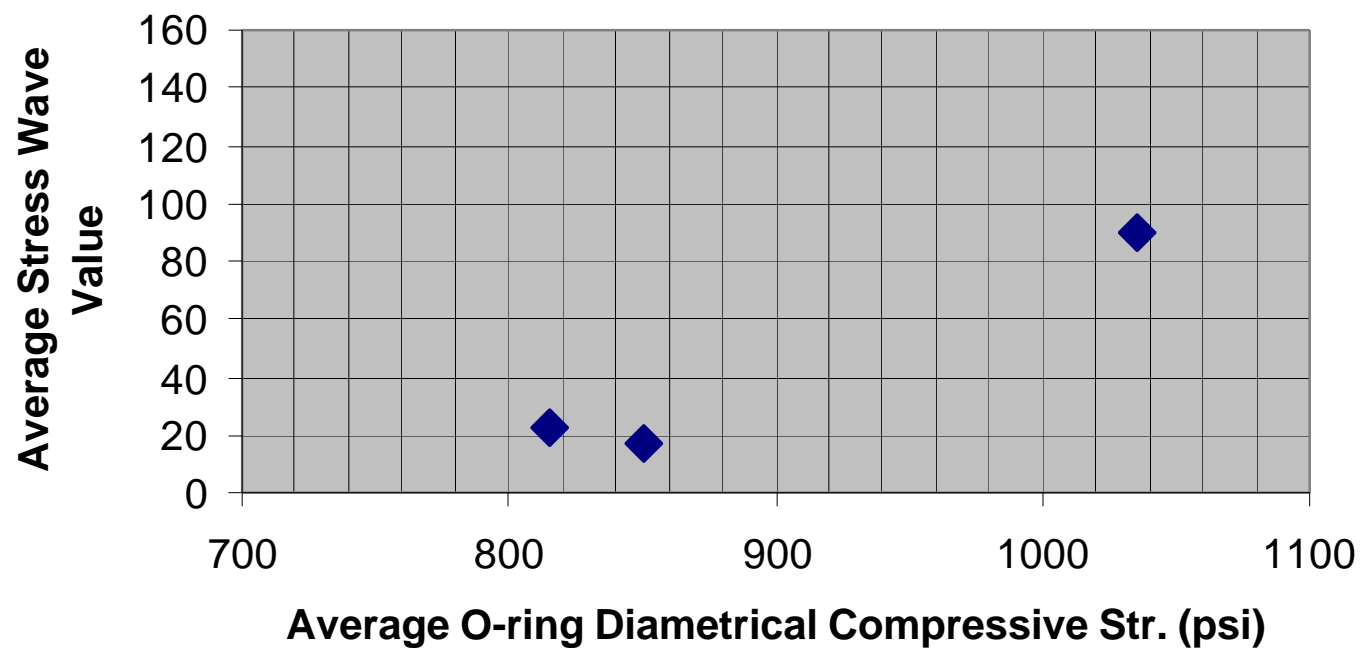

Figure 106 - Preliminary NDE results on PRD-66 hot gas filters, comparing ANL's Stress Wave Value to the o-ring diametrical compressive strength of the same filter. 
ANL also conducted their acousto-ultrasound test on a 1.5-meter, as-manufactured filter. Measurements were taken along the length of the filter as well as around the circumference. The data is shown in Figure 107. Unfortunately, this filter broke in the middle during shipping, and the data points in the center of the location are estimated from the measured SWF on either side of the break. The "composite average" means that readings were taken at three radial positions at each axial location, and the average for each location was plotted. The data seems to indicate a trend in the SWF along the length of this filter. Some radial variability was also observed; the average standard deviation for readings at each location was 29 . At the time this report was published, this filter had not been cut up and tested for mechanical properties.

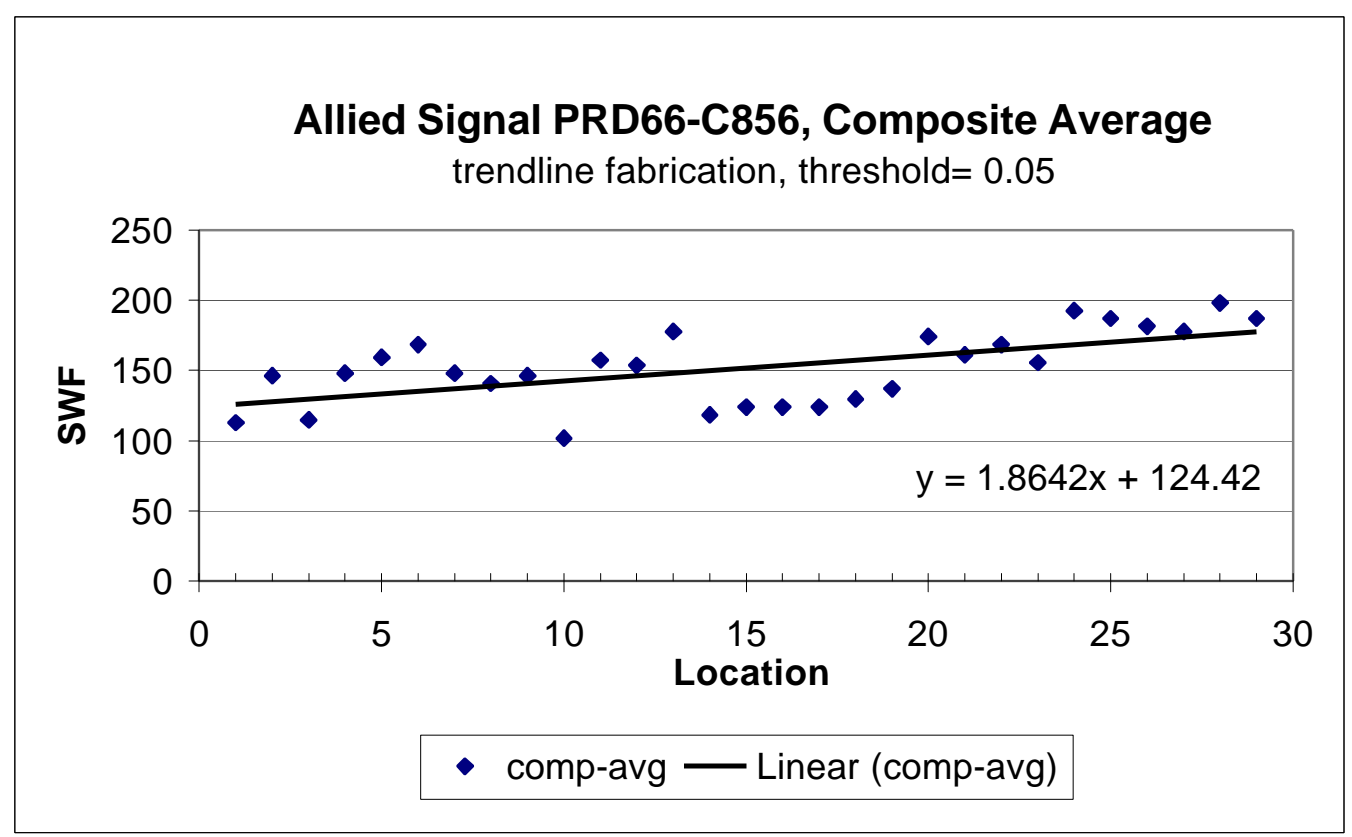

Figure 107 - Stress Wave Factor (SWF) trend along the length of PRD-66 filter C856

Although this measurement technique may be of use in predicting the mechanical strength of PRD-66C filters, much more data will be required to determine if a useful correlation exists. It may also be feasible to use this device to detect localized problems, such as an internal delamination, but testing of filters with known defects will be required. 


\section{CONCLUSIONS}

\section{GENERAL}

Inherent thermal shock resistance and low cost raw materials made PRD-66 a promising candidate for a hot gas filtration applications. The support and funding provided by NETL, however, was critical to the implementation of needed product and process improvements. The final product is now ready to serve the filtration needs of advanced coal combustion systems and provide reliable performance despite the thermal upsets that frequently accompany the start-up of a new facility.

TASK 3

1. Under the initial objectives of Task 3, product modifications were conducted and evaluations were performed on the "baseline" PRD-66 Hot Gas Filter.

- Filters were produced which had lower backpressure, good membrane adhesion and a stronger flange region.

- These filters passed permeability and "particle collection efficiency" tests conducted by Westinghouse Science and Technology Center (W-STC).

- Strength characterization of the filter material, conducted by W-STC and by DLC, deemed PRD-66 to have sufficient strength for PFBC applications.

- The feasibility of producing a wound ("yarn only") membrane on the inside diameter of the filter was demonstrated.

2. Independent field trials of the "baseline" PRD-66 filter, at American Electric Power's Tidd Facility, suggested that inadequacies existed in the membrane and the underlying support wall. These problems would not have been corrected by the modifications under evaluation at that time. More radical changes were required and evaluated.

3. Modifications to the alumina slurry composition were effective at reducing the interlaminar voids within the wall of the filter element.

4. A new DLC lab-scale test procedure (PIT) was developed to evaluate the membrane integrity of 2" long specimens at room temperature. Once it was possible to differentiate between "good" membranes and "poor" membranes, membrane experiments could be conducted. 
5. A preferred membrane construction, which combined a wound slurry-coated yarn and an particulate alumina, produced the best combination of good surface filtration and low backpressure.

6. The preferred membrane construction was fine-tuned, and two types were selected for continued evaluation.

- $\quad$ PRD-66C - nominal $25 \mu$ pore size

- $\quad$ PRD-66M - nominal $10.5 \mu$ pore size

7. Significant progress was made in developing a membrane coating for the inside surface of PRD-66 filters.

8. Both PRD-66C and PRD-66M Hot Gas Filters successfully passed high temperature and high pressure (HTHP) tests conducted by Westinghouse.

9. PRD-66C filters were evaluated in pressurized circulating fluidized bed (PCFBC) conditions in Foster Wheeler's Karhula facility for 691 hours.

- Throughout the testing, no in-process failures, no delaminations, no cracking, and no "divots" occurred.

- Examination of the cross-section of exposed filters confirmed that the elements had provided effective surface filtration.

- Exposed filters proved to be both chemically and physically stable, as determined by evaluating strength, composition, and microstructure.

10. PRD-66C filters were evaluated in a combustion environment at Southern Company Services' Power System Development Facility, in Wilsonville, Alabama.

- Several PRD-66C filters accumulated over 1,900 hours of exposure.

- PRD-66C filters survived two severe thermal shock events, which caused the catastrophic failure of a wide variety of monolithic ceramic filters.

- Membrane spalling occurred on two filters do to an unusual sensitivity to the rigors of installation.

- Problems with the contour specifications of the PRD-66 flange were responsible for cracks in the tapered regions of two filters. 
- Three filters broke at the base of the filter nut, the reason for which has not been conclusively proven. The problem is believed to stem from the vibration of the filter coupled with a deficiency in product strength and the specification for the length of the "strengthened region" at the open-end of the filter.

\section{TASK 4}

1. A raw materials plan was completed which found that the quality assurance provided by our suppliers was adequate for the needs of PRD-66 filter manufacturing.

2. All critical in-process instrumentation and calibration procedures were reviewed; improvements were implemented where necessary.

3. An analysis of process sensitivity, as it related to the WINDING OF THE FILTER, was conducted at the extremes of the normal process limits.

- Product quality was stable within normal process limits except for a slight decrease in alumina pickup when the slurry viscosity was very low. The "low-viscosity limit" was raised.

- Winding interruptions of less than fifteen minutes had no impact on product quality, unless the relative humidity of the winding environment fell below the normal process limit. This allowable "window" makes it possible to use "short" bobbins of feed yarn without risk to the quality of the filter.

4. An analysis of process sensitivity, as it related to the fabrication of the filter membrane, was conducted at the extremes of the normal process limits.

- Slightly higher amounts of fusible binder improved the adhesion of the Type-C particulate membrane.

- The backpressure of the filter was insensitive to normal variations in amount of particulate membrane applied.

- Cracks in the membrane occasionally resulted where the particulate membrane was noticeable "too thick".

- A few extremely fine cracks in the Type-M membrane were common in most PRD$66 \mathrm{M}$ filters, when examined in transmitted light. 
5. A reasonable $70 \%$ yield was demonstrated during a process capability run of thirty filters made to the specifications required by the Westinghouse Advanced Particulate Filtration System.

- A variety of equipment modifications were implemented throughout the "capability demo" which improved processability, including different mandrel designs and a different filter cutting technique.

- The "length of the flange" and the "inside diameter of the flange" were the most difficult specifications to meet.

- The equipment utilization was well below expectations due to a high level of maintenance and repair required for the prototype winders.

6. Significant progress was made in modifying the prototype winding equipment to increase reliability and demonstrate several features necessary for increased production quantities.

\section{TASK 5}

1. Fifty filters were produced using multiple winding stations.

2. Observed increased variability in filter dimensions and properties, along with a reduced yield, compared to the filters produced by one winding station in Task 4.

3. Twelve filters were provided for field testing in a combustion environment at Southern Company Services' Power System Development Facility, in Wilsonville, Alabama

4. Eight filters were provide for field testing in a gasification environment at the Wabash River Coal Gasification Repowering Project, near Terre Haute, Indiana 


\section{RECOMMENDATIONS}

1. Additional exposure of PRD-66C Hot Gas Filters is recommended in Advanced Coal-Based Power programs, particularly those being sponsored by the DOE. Additional PFBC field experience is necessary to determine their long-term potential.

2. In an effort to improve the reliability of PRD-66 Hot Gas Filters, it is recommended that Honeywell Advanced Composites implement the following changes to production protocols:

- Any new batches of raw materials should be use to make several qualification filters. Large-scale use of this material should be determined by the strength of these filters. Samples of all new raw lots of alumina should be characterized for particle size distribution. Samples of all raw material lots should be retained indefinitely

- The dimensional specifications for PRD-66C flanges in Siemens Westinghouse filtration systems should be changed, specifically a shortening of the nominal length by $2 \mathrm{~mm}$.

- The "strengthened" region at the open-end of the PRD-66C filter should be lengthened by one inch.

3. It is recommended that Honeywell Advanced Composites evaluate the following:

- A modified flange contour to minimize localized stresses.

- A nondestructive evaluation, which can be used to predict strength or find localized flaws in PRD-66 filters.

- A more humid winding environment to increase product strength

4. It is recommended that users of PRD-66 filters:

- Modify handling techniques, wherever feasible, to reduce impact damage to the filters.

- Continue to study the vibrational characteristics of their systems. 


\section{ACKNOWLEDGMENTS}

The authors and Honeywell Advanced Composites Inc. gratefully acknowledge the contributions of several individuals whose support has been vital to this program.

We appreciate the technical guidance and assistance of Ted McMahon, our DOE-NETL COR, as well as his support, throughout this program.

Mary Anne Alvin of Westinghouse Science and Technology Center made significant contributions to our understanding of the field performance of our products, and provided valuable feedback on our product modifications.

During the failure analysis of the Tidd-5 filters, we were extremely fortunate to have technical assistance from Rich Dennis and Duane Smith of NETL, Tom Lippert and Rich Newby of Westinghouse Science and Technology Center, Tina Watne and John Holmes of UND's Energy and Environmental Research Center, and Dick Tressler of Penn State. Their knowledge, experience and creativity provided an excellent foundation for the work in this task.

We are grateful for the assistance of Juhani Isaksson, Reijo Kuivalainen \& Timo Eriksson, of Foster Wheeler Energia Oy. The exposure of our material at their Karhula R\&D Center, was PRD-66C's first field trial, and their input was critical to understanding our product's performance.

The staff of Southern Company Services' Power Systems Development Facility played a vital role in the evolution of the PRD-66 Hot Gas Filter. Howard Hendrix and Xiaofeng Guan, along with Jack Spain at Southern Research Institute, and were instrumental in helping us to understand the behavior of PRD-66 filters in the field. We truly valued their contribution.

We appreciated the assistance of Bill Ellingson and Dick Koehl at Argonne National Lab and Roger Chen at West Virginia University for their efforts in characterizing the PRD-66 filters by non-destructive means. Their techniques show great promise.

Finally, we wish to acknowledge Jeffrey Chambers, our former program manager. Before assuming new responsibilities within DuPont, he had developed a comprehensible technical agenda that made the achievements of this program possible. 


\section{REFERENCES}

1. M.A. Alvin, R.E. Tressler, T.E. Lippert, and E.S. Diaz, "Evaluation of Ceramic Filter Material, Selection for Application," Proceedings of the Coal-Fired Power Systems '93 Advances in IGCC and PFBC Review Meeting. DOE/METC-93/6131 (DE93000289) June 1993.

2. M.A. Alvin, T.E. Lippert, E.S. Diaz, and E.E. Smeltzer, "Filter Component Assessment," Proceedings of the Advanced Coal-Fired Power Systems '95 Review Meeting - Volume I. DOE/METC-95/1018 (DE95009732) June 1995.

3. M.A. Alvin, "Filter Component Assessment," Monthly Status Report, December'96, DOE DE-AC21-94MC31147 December 1996.

4. M.A. Alvin, "Advanced Ceramic Materials for Use in High Temperature Particulate Removal Systems," Ind. Eng. Chem. Res. 1996, 35, 3384-3398.

5. M.A. Alvin, T.E. Lippert, E.S. Diaz, E.E. Smeltzer and R.E. Tressler, "Durability of Ceramic Filters," Proceedings of the Advanced Coal-Fired Power Systems '94 - Advances in IGCC and PFBC Review Meeting, 545-571, DOE/METC-94/1008.

6. M.A. Alvin, T.E. Lippert, E.S. Diaz, E.E. Smeltzer, and G.J. Bruck, "Filter Component Assessment," Proceedings of the Advanced Coal-Fired Power Systems '97 Review Meeting, Pittsburgh, PA, July 22-24, 1997.

7. J.A. Chambers, "Development and Testing of PRD-66 Hot Gas Filters," Proceedings of the Advanced Coal-Fired Power Systems '95 Review Meeting - Volume II. DOE/METC-95/1018 (DE95009732) June 1995.

8. J.A. Chambers, "Performance of PRD-66 Hot Gas Candle Filters in the AEP/Tidd PFBC Facility," Proceedings of the Advanced Coal-Fired Power Systems '96 Review Meeting. July 16-18, 1996.

9. M. Davidson, E. Galloway, X. Guan, H. Hendrix, R. Read, P. Scarborough, R. Dahlin, and C. Landham, "Combustion Testing of the Westinghouse Advanced Particle Filter at the Power Systems Development Facility (PSDF)," Symposium on High-Temperature Particulate Cleanup for Advanced Coal-Based Power Systems, Birmingham, AL, April 20-23, 1998.

10. O.M. Jadaan, D.L. Shelleman, J.C. Conway Jr., J.J. Mecholsky Jr., and R.E. Tressler, "Prediction of the Strength of Ceramic Tubular Components: Part I - Analysis," Journal of Testing and Evaluation, JTEVA, Vol. 19, No. 3, May 1991, pp. 181-191.

11. ASTM Designation: C 1239-93, Standard Practice for Reporting Uniaxial Strength Data and Estimating Weibull Distribution Parameters for Advanced Ceramics, Published June 1993.

12. E.S. Connolly and G.D. Forsythe, "Advanced Hot Gas Filter Development", Task 3 and Task 4 Topical Report, December 22, 1998, DOE/FETC Contract No. DE-AC21-94MC31214, Appendix 3: MA Alvin, “Advanced Hot Gas Filter Development:”, Subcontractor's Summary Report, March 31, 1998

13. M. Davidson, E. Galloway, X. Guan, H. Hendrix and P. Scarborough, "Overview of PCD Test Program", Filter Element Workshop, Wilsonville, AL, November 1998.

14. M.A. Alvin, "Hot Gas Filter Development and Performance", High Temperature Gas Cleaning Symposium, Karlsruhe, Germany, September 1999. 
15. J.D. Spain and H.S. Starrett, "Preliminary Evaluation of Candidate Candle Filter Materials", Proceedings of Advanced Coal-Based and Environmental Systems '98, Morgantown, WV, July 1998

16. R.A. Wagner, "Ceramic Composite Hot Gas Filter Development", Proceedings of Advanced Coal-Based and Environmental Systems'98, Morgantown, WV, July'98

17. Roger H.L. Chen and A.C. Kiriakidis, "Nondestructive Evaluation of Ceramic Candle Filters Using Vibration Response", Proceedings of Advanced Coal-Based and Environmental Systems'98, Morgantown, WV, July'98

18. W.A. Ellingson, E.R. Koehl, J.G. Sun, C. Deemer, H. Lee, and T. Spohnholtz, "Development of NDE Methods for Hot Gas Filters", U.S. Department of Energy, Office of Fossil Energy/Advanced Research and Technologies/Materials Program and Hot Gas Clean-Up Program under Contract W-31-109-Eng-38, Nov'99

19. E.S. Connolly and G.D. Forsythe, “Advanced Hot Gas Filter Development”, Task 3 and Task 4 Topical Report, December 22, 1998, DOE/FETC Contract No. DE-AC21-94MC31214

20. M.J. Hickey and T.A. Lynch, "Particulate Filters at the Wabash River Coal Gasification Repowering Project", Proceedings of Advanced Coal-Based and Environmental Systems'98, Morgantown, WV, July'98 
Appendix 1

United States Patent 5,902,363

CERAMIC HOT-GAS FILTER 


\section{United States Patent}

[11] Patent Number:

[45] Date of Patent:
$5,902,363$

May 11, 1999

\section{[54] CERAMIC HOT-GAS FILTER}

[75] Inventors: Elizabeth Sokolinski Connolly, Wilmington, Del.; George Daniel Forsythe, Landenberg, Pa.; Daniel Matthew Domanski, New Castle; Jeffrey Allen Chambers, Hockessin, both of Del.; Govindasamy Paramasivam Rajendran, Boothwyn, Pa.

[73] Assignee: AlliedSignal Composites Inc., Newark, Del.

[21] Appl. No.: 08/896,372

[22] Filed: Jul. 18, 1997

[51] Int. Cl. ${ }^{6}$ B01D 39/20; C04B 41/50

[52] U.S. Cl. $\mathbf{5 5} / \mathbf{4 8 7} ; 55 / 523 ; 55 / 527$

[58] Field of Search $55 / 523,527,486$, $55 / 487,529,498$

$[56]$
References Cited

\section{U.S. PATENT DOCUMENTS}

3,843,561 10/1974 Sobel .............................. 252/465

3,949,109 4/1976 McBride ................................ 428/36

3,986,528 10/1976 Green .................................. 138/177

4,092,194 5/1978 Green ................................. 156/89

$4,629,483 \quad 12 / 1986$ Stanton ................................. 55/487

$4,732,594 \quad 3 / 1988$ Mizrah et al. .............................. $55 / 523$

4,746,341 5/1988 Komoda ................. 55/487

$4,810,273 \quad 3 / 1989$ Komoda ..................................... 55/487

$4,846,906 \quad 7 / 1989$ Helferich et al. ........................ 156/89

$4,889,630 \quad 12 / 1989$ Reinhardt et al. ..................... 210/490

4,946,487 8/1990 Butkus .................................. 55/523

4,968,467 11/1990 Zievers ................................ 264/62

4,976,760 12/1990 Helferich et al. ........................ 55/487

$5,071,457$ 12/1991 Schmidt, Jr. et al. .................. 55/523

\begin{tabular}{|c|c|c|}
\hline & & . \\
\hline & & orsythe .......... \\
\hline & & ndo et al. \\
\hline & 2 & $55 / 52$ \\
\hline & $12 /$ &. .502 \\
\hline & & $428 /$ \\
\hline & & .55 \\
\hline & & \\
\hline & $10 / 1$ & 55 \\
\hline & & \\
\hline 60 & $2 / 1997$ & Connolly et al. \\
\hline
\end{tabular}

\section{FOREIGN PATENT DOCUMENTS}

0216729 4/1987 European Pat. Off. .

Primary Examiner-Duane S. Smith Attorney, Agent, or Firm - Larry J. Palguta

[57]

ABSTRACT

A ceramic hot-gas candle filter having a porous support of filament-wound oxide ceramic yarn at least partially surrounded by a porous refractory oxide ceramic matrix, and a membrane layer on at least one surface thereof. The membrane layer may be on the outer surface, the inner surface, or both the outer and inner surface of the porous support. The membrane layer may be formed of an ordered arrangement of circularly wound, continuous filament oxide ceramic yarn, a ceramic filler material which is less permeable than the filament-wound support structure, or some combination of continuous filament and filler material. A particularly effective membrane layer features circularly wound filament with gaps intentionally placed between adjacent windings, and a filler material of ceramic particulates uniformly distributed throughout the gap region. The filter can withstand thermal cycling during backpulse cleaning and is resistant to chemical degradation at high temperatures.

19 Claims, 3 Drawing Sheets 


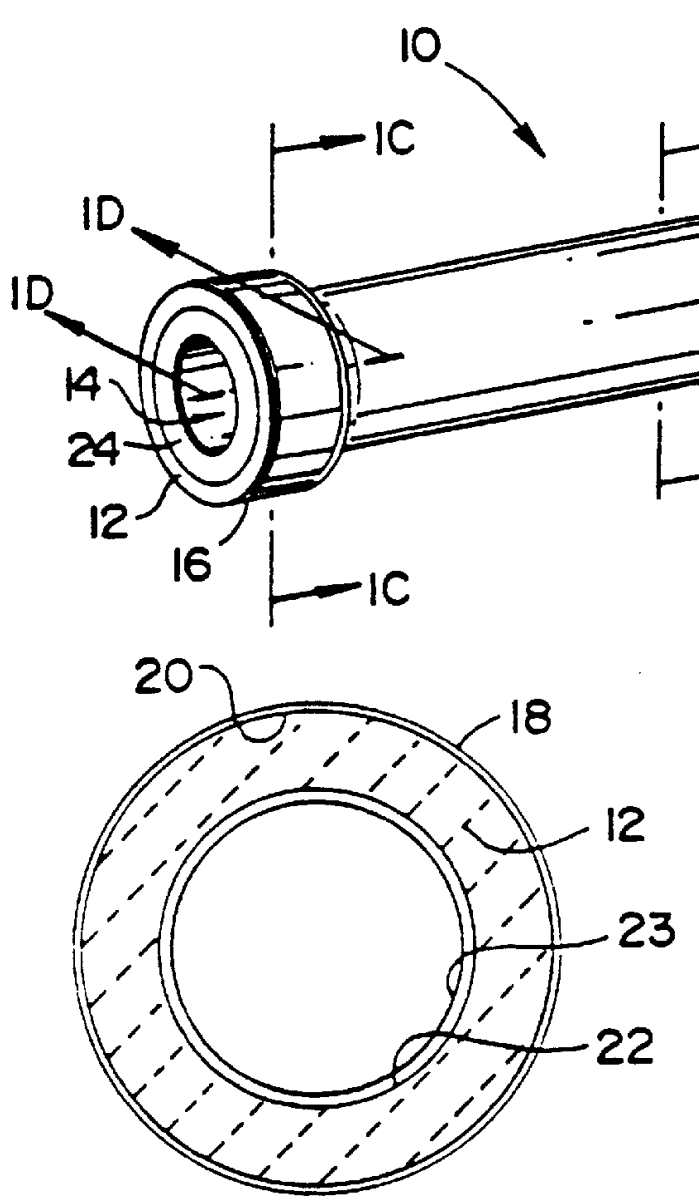

$F|G|$.

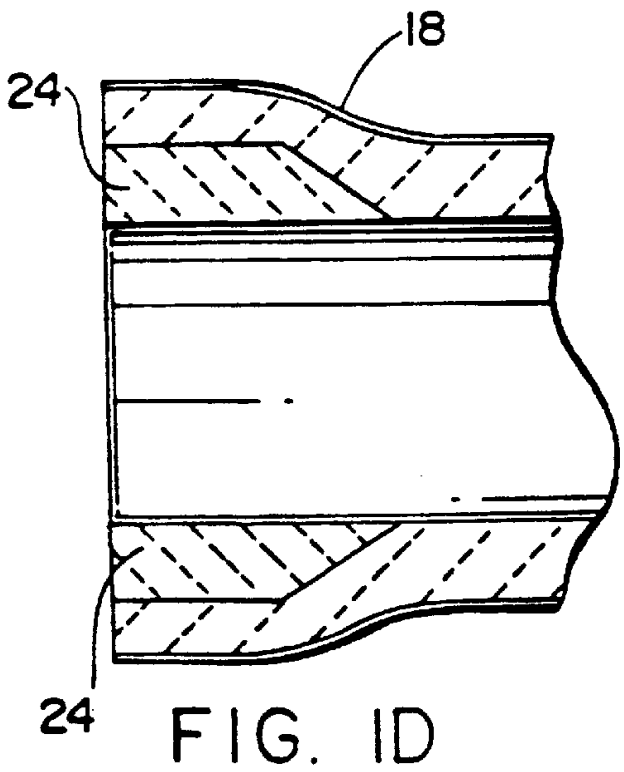

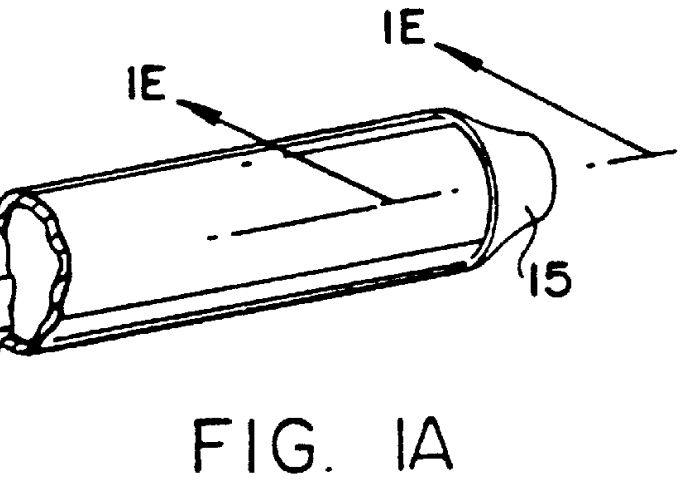

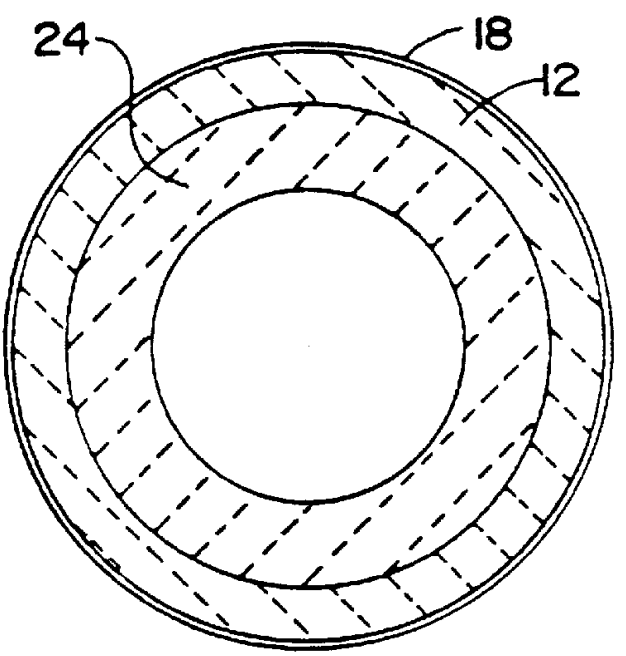

FIG. IC

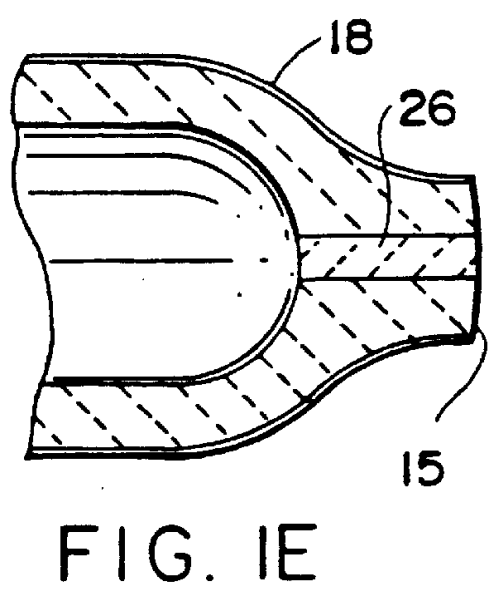




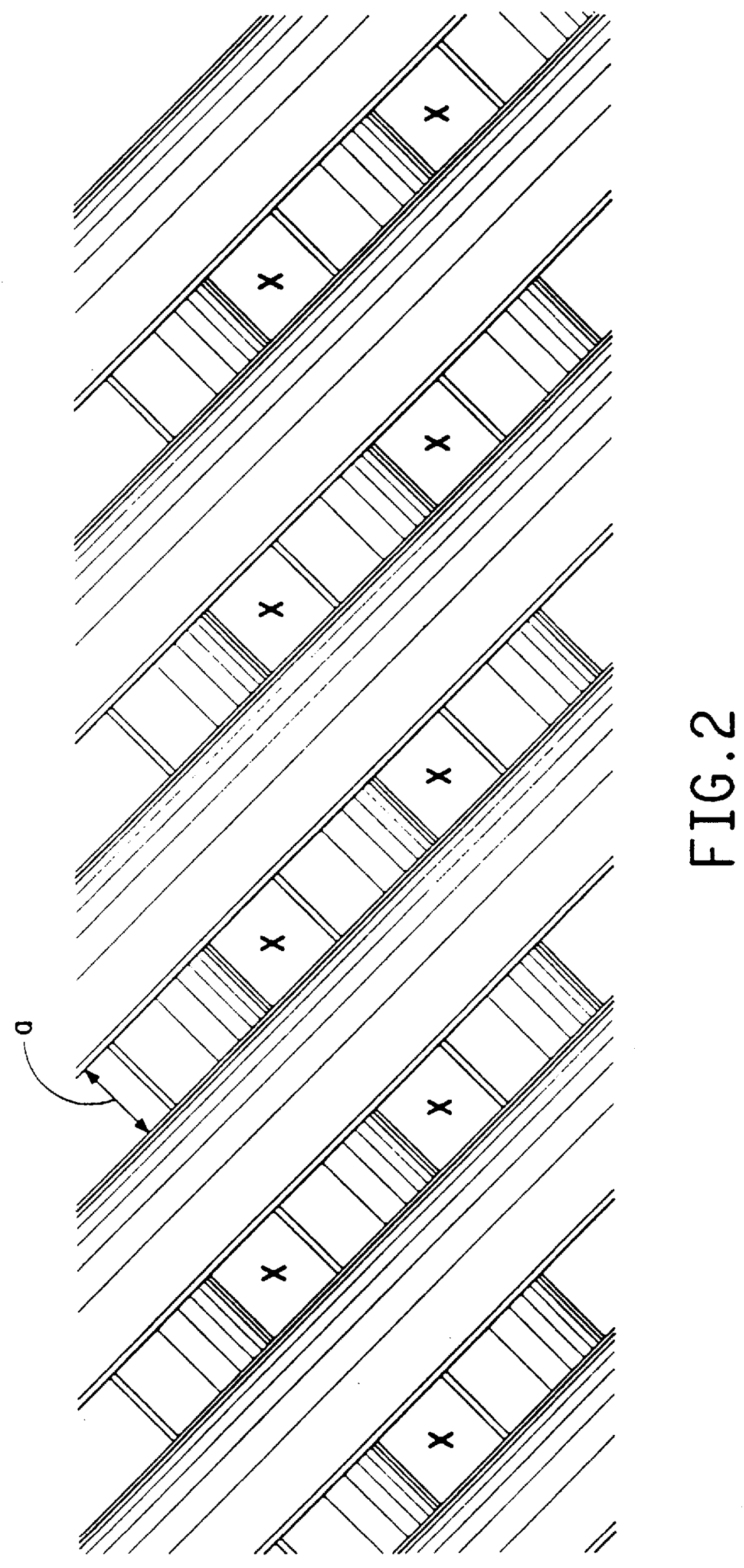



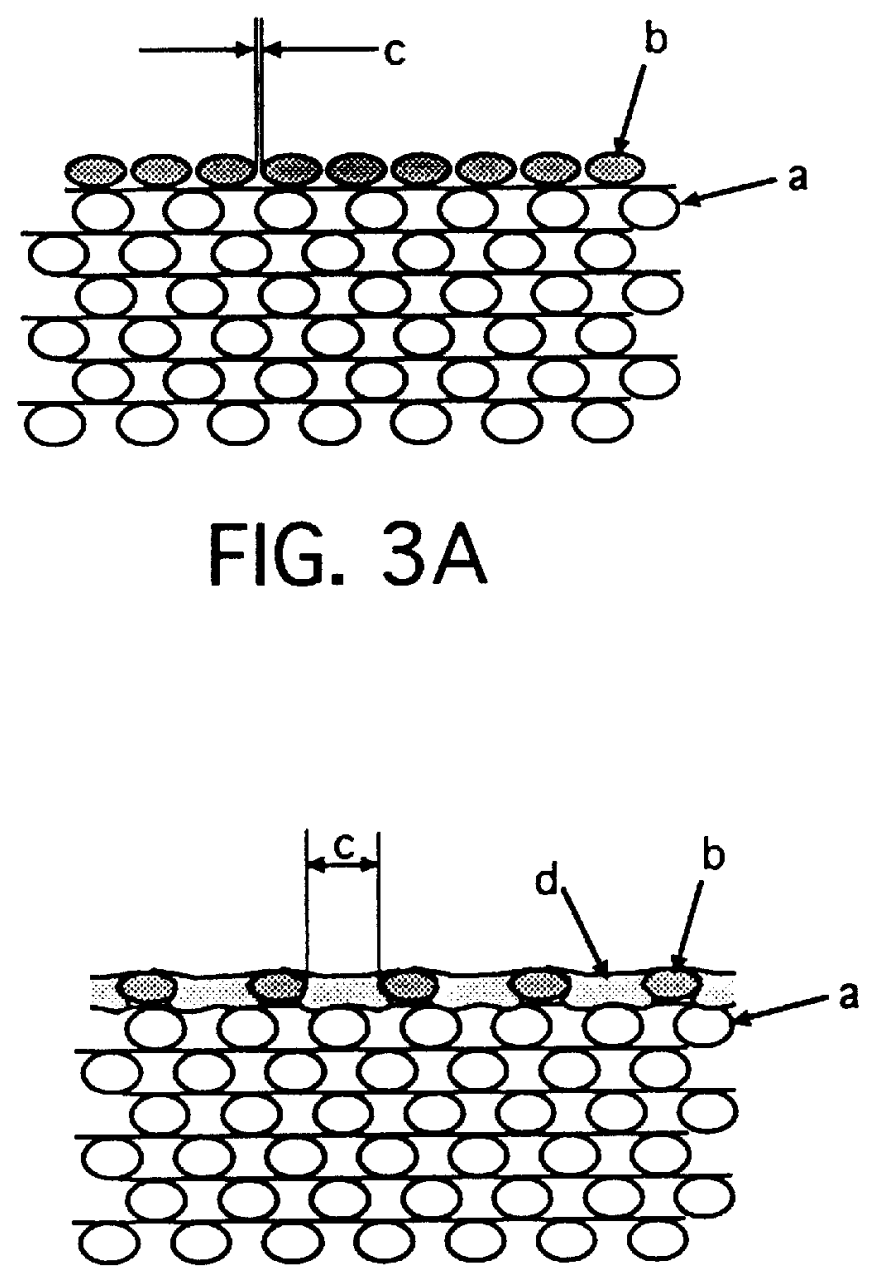

FIG. 3B

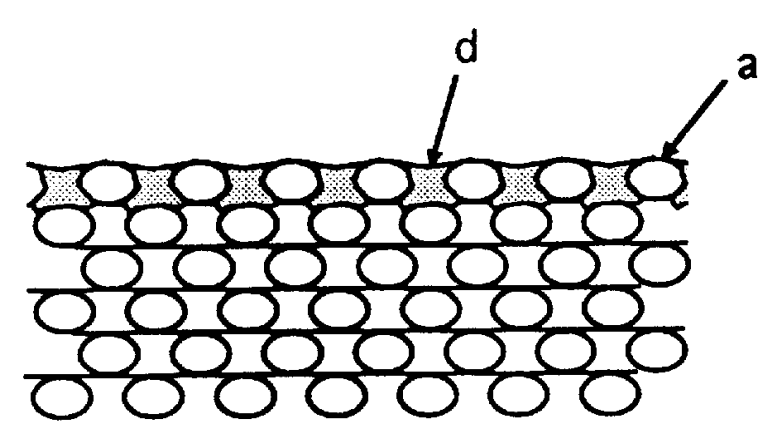

FIG. 3C 


\section{CERAMIC HOT-GAS FILTER}

This invention was made with Government support under Contract No. DE-AC21-94MC31214 awarded by the Department of Energy. The Government has certain rights to this invention.

\section{FIELD OF THE INVENTION}

The present invention relates to a composite ceramic candle filter for removing particulates from a hot gas stream, and a method for making said filter.

\section{DESCRIPTION OF RELATED ART}

Ceramic filters have been tested in processes such as coal gasification and coal combustion to remove particulates from hot flue gases to protect downstream equipment from corrosion and erosion and to comply with EPA NSPS (New Source Performance Standards) regulations. Ceramic filters in a tubular (candle) form, with one end closed and the other end open have been shown to remove the particulates efficiently. The hot gas to be filtered typically flows from the outside to the inside of the filter, with particulate-free gas exiting from the open end. The candle geometry is also suited for removal of the filtered cake by backpulsing with compressed gases.

Ceramic hot-gas candle filters must withstand exposure to chemically corrosive gas streams at temperatures in excess of $800^{\circ} \mathrm{C}$. In addition, they are subjected to significant thermal stresses during backpulse cleaning which can cause catastrophic failure of the ceramic candle filter element.

Ceramic hot-gas candle filters known in the art are generally fabricated from either porous monolithic materials or porous ceramic fiber-containing composite materials. Monolithic ceramic candle filters are either weak or can fail catastrophically in use. Composite filters are less susceptible to catastrophic failure and generally have improved strength, toughness, and thermal shock resistance versus monolithic ceramic filters.

Candle filters may have relatively uniform porosity throughout the filter or they may comprise a porous support with a thin layer, or membrane, of fine porosity on the outer surface of the support. The membrane layer is typically applied to the filter using a variety of methods such as coating from a dispersion containing finer grains than those used in the support for smaller membrane pore sizes, bonding randomly arranged chopped ceramic fibers to the support using colloidal (or sol) materials, or forming a ceramic matrix by chemical vapor infiltration.

Materials used to fabricate ceramic hot-gas filters generally include oxides such as aluminosilicates, glass, and alumina, and non-oxides such as silicon carbide and silicon nitride. Oxide-based ceramic filters have adequate resistance to flue gas atmospheres and fly-ash for the design life of the filters; however, they generally have low thermal shock resistance. Non-oxide ceramics generally have good thermal shock resistance, however they are susceptible to oxidation in the corrosive environment to which they are subjected which results in a degradation of mechanical properties.

The disadvantages of ceramic candle filters known in the art include failure, often catastrophic, due to thermally induced stresses caused by backpulse cleaning, chemical degradation caused by species present in the hot gases being filtered, delamination of the membrane layer, incomplete removal of the filter cake upon backpulsing, and high cost. They also tend to be heavy, requiring expensive support structures to hold an array of the candles in the filter unit.

\section{SUMMARY OF THE INVENTION}

The present invention is directed to a ceramic hot gas filter comprising a porous elongated filter support and a porous membrane layer on at least one surface thereof. Specifically, the porous membrane may be on the outer surface, the inner surface, or both the outer and inner surface of the porous elongated filter support. The membrane layer(s) is firmly adherent to the support and therefore does not suffer from delamination problems. The porosities of the support and membrane are controlled such that the support functions as a bulk filter and the membrane layer functions as a surface filter. The support has an opening at one end into a hollow interior, a closed end opposite the open end, and an external flange integral with the open end. The support is formed of a plurality of layers of oxide ceramic support yarn, each layer being arranged in a crisscrossing relationship with neighboring layers to form a plurality of quadrilateralshaped openings. The yarn in the support is coated with a first oxide ceramic material which, upon heat treatment, forms a porous refractory oxide support matrix. The membrane layer(s) may be formed of an ordered arrangement of continuous filament oxide ceramic membrane yarn, a uniform coating of ceramic filler material, or some combination ${ }_{5}$ of the two. Any yarn present in the membrane layer is (preferably prior to winding) coated with a second oxide ceramic material which, upon heat treatment forms a porous refractory oxide membrane matrix. Preferably, the support yarn and the continuous filament membrane yarn each 30 contain at least 20 weight percent alumina $\left(\mathrm{Al}_{2} \mathrm{O}_{3}\right)$ and have softening points above about $750^{\circ} \mathrm{C}$. The ceramic coating materials are generally particulates of oxides or oxide compounds, or mixtures thereof and may also include oxide precursor materials. The membrane layer(s) has a porosity ${ }_{5}$ that is less than that of the support. Preferably the quadrilateral-shaped openings have dimensions of about 100 to about 500 microns after heat treatment so that the support functions as a bulk filter. The membrane layer(s) preferably has pore diameters of about 0.1 to 50 microns and functions as a surface filter. In a preferred embodiment of the invention, the support yarn has generally the same composition as the membrane yarn and the support matrix has generally the same composition as the membrane matrix.

The present invention also provides a method for making 45 a ceramic hot gas filter involving the steps of fabricating an elongated porous filter support by coating a ceramic oxide support yarn with a first coating composition, winding the coated support yarn onto a mandrel to form a plurality of layers of the coated support yarn, each layer being arranged $5_{0}$ in a crisscrossing relationship with neighboring layers to form a plurality of quadrilateral-shaped openings. The mandrel may be contoured to provide an integral external flange adjacent to one end of the support. Alternatively, a separate collar insert may be slid onto a uniformly cylindrical man5 drel to form the flange portion of the support. The resulting support has an open end adjacent to the flange, an outside surface, and a second open end opposite the flanged end.

A membrane layer is then formed on at least one surface of the support. For example, the membrane layer may be 60 formed on the outer surface by coating a continuous filament oxide ceramic membrane yarn with a second coating composition and applying the coated membrane yarn in an ordered arrangement on the outer surface of the support. Methods for forming the ordered arrangement membrane 5 layer(s) include hoop winding a single yarn, multiple yarn winding, fabric wrapping and coating with a particulate slurry or a solution containing ceramic precursor materials. 


\section{3}

In a preferred embodiment, the ordered arrangement comprises a circular or hoop winding of the continuous filament oxide ceramic so as to define a gap of predetermined width between adjacent windings. The gap is then filled with additional ceramic filler material, preferably an oxide material, which upon subsequent heat treatment, forms a porous refractory membrane matrix. The width and uniformity of the gap between adjacent hoops or windings is not particularly critical; however, uniform filling of the gap with filler material is desirable, both around the circumference 10 and along the length of the filter. In another embodiment, a membrane layer is formed by winding the coated continuous filament such that adjacent hoops or windings are as close to one another as possible and no such filler material is applied. Yet another embodiment features a membrane layer comprising ceramic filler material but without hoop-wound filaments; i.e., an infinitely large gap between hoop windings. In this embodiment, for example, ceramic particulates, preferably of an oxide material and preferably in the form of a slurry, are applied to the support layer as uniformly as 20 possible, so as to essentially close off the diamond shaped openings formed by the crisscrossing filaments of the support layer. A slurry is a convenient form for the filler material because the slurry is amenable to being painted by brush or spray, or being dip coated, etc. Another useful form for 25 providing the ceramic filler material to the developing candle filter is as a paste, which may then be applied using, for example, a spatula-like flexible applicator. Other media for communicating the filler material to the developing candle filter may occur to an artisan of ordinary skill and should be considered therefore to be within the scope of the present invention.

Once the support layer has been wound, the support mandrel removed, and the membrane layer(s) formed, the second open end (opposite the flange end) is closed using an 35 oxide ceramic material. The support and membrane layer(s) are heat treated to convert the first coating composition to a porous refractory oxide support matrix and to convert the various coating compositions to a porous refractory oxide membrane matrix.

The present invention provides a strong, lightweight ceramic hot-gas candle filter which has a greater than $99.5 \%$ particulate collection efficiency, thus meeting EPA NSPS regulations. Failure of the filter is generally not catastrophic since if the membrane is damaged, the support quickly blinds at the location of the damage due to its bulk filtration properties, thus preventing release of particulates and protecting downstream process equipment such as gas turbines or sorbent beds. The filter of the present invention is resistant to chemical degradation due to the oxide compositions used, 50 and at the same time provides excellent thermal shock resistance which is not generally typical of oxide materials. The smoothness of the membrane surface(s) results in efficient removal of the filter cake during backpulse cleaning. In addition to the above-mentioned advantages, the filter 55 of the current invention is potentially low cost relative to most of the commercially available candle filters.

\section{DEFINITIONS}

"Ceramic" as used herein means crystalline or partially crystalline materials, or non-crystalline glasses, which comprise essentially inorganic, nonmetallic substances.

"Continuous fiber or filament" as used herein means a fiber or filament having a length which is at least 1000 times the diameter of such fiber or filament.

"Filler material" or "membrane filler material" as used herein means those bodies in the membrane layer other than

\section{4}

those bodies making up the yarn or any slurry material coated on the yarn. As such, the filler material may be in the form of powders, particulates, whiskers, chopped fibers, platelets, flakes, spheres, tubules, pellets, etc.

"Membrane" or "membrane layer" as used herein refers to that layer which is deposited or applied onto at least one surface of the support layer, has a lower porosity than the support layer, and which provides the majority of the filtering action

10 "Oxide" as used herein is meant to include oxides, oxide compounds (e.g. mullite, spinel), or precursors thereof.

"Support" or "support layer" as used herein refers to the structure formed by winding single or multiple continuous ceramic fiber or filament around a mandrel in a crisscrossing arrangement to produce an ordered array of diamond shaped openings. The function of the support or support layer is to provide a suitably strong foundation to which the membrane adheres.

\section{BRIEF DESCRIPTION OF THE DRAWINGS}

FIG. 1A is a schematic perspective view of an embodiment of a filter element of the current invention, including an optional flange collar section.

FIG. $1 \mathrm{~B}$ is a cross section of the filter element taken on line $1 \mathrm{~B}-1 \mathrm{~B}$ of FIG. $1 \mathrm{~A}$.

FIG. $1 \mathrm{C}$ is a cross section of the flange section taken on line $1 \mathrm{C}-1 \mathrm{C}$ of FIG. 1A.

FIG. 1D is a cross section of the flange section taken on line 1D-1D of FIG. 1A.

FIG. 1E is a cross section of the closed end taken on line 1E-1E of FIG. 1A.

FIG. 2 shows openings formed by the overlap of two 35 layers of yarn in a support layer comprising an embodiment of the present invention.

FIGS. 3A, 3B, and 3C are cross-sectional views of the filter wall which illustrate the construction variations of the membrane layer. 40

\section{DETAILED DESCRIPTION OF THE INVENTION}

The hot-gas filter of the current invention is of the candle 5 filter type and comprises a porous ceramic support having a porous ceramic membrane layer on at least one surface thereof. Specifically, the porous membrane may be on the outer surface, the inner surface, or both the outer surface and inner surface of the porous ceramic support. The membrane is less porous than the support and serves as a surface filter, preventing pollutant particulates from penetrating therethrough. The support has good filtration capacity for fly-ash and serves as a bulk filter, capable of trapping particulates between its inner and outer surface, should membrane 5 leakage occur.

Referring to FIGS. 1A-1E, the filter 10 comprises a support 12 having a generally elongated tubular shape with an open end 14 at one end into a hollow interior. The end $\mathbf{1 5}$ of the support opposite the open end is generally closed. The 60 support further includes an external flange 16 integral with the open end $\mathbf{1 4}$ which supports the filter in a tube sheet in use. The flange may also include an optional collar insert 24, integral with the flange, and described in more detail below. The membrane layer(s) 18, 23 are formed on the outer 65 surface $\mathbf{2 0}$ of the support and/or the inner surface $\mathbf{2 2}$ of the support. End $\mathbf{1 5}$ is generally closed by filling with a ceramic material 26, and the flange section 16 and tip section of the 
5

support adjacent the closed end $\mathbf{1 5}$ are made impervious as described below.

The overall porosity of the support layer is determined by a combination of the open volume created by the diamond or parallelogram-shaped openings (macropores) and the porosity of the matrix coating surrounding the individual yarns (micropores). The porosity of the membrane layer is due primarily to the porosity between adjacent particles making up the layer (micropores) or due to microcracks.

The macroporosity of the support may be calculated from the volume of the support (calculated from the measured dimensions of the support), the weight of the support, and the bulk density of the support (fiber and matrix, including any microporosity). The bulk density is measured using mercury porosimetry.

The matrix is applied in such a way that the channels in the support are not substantially closed. The matrix generally imparts integrity and mechanical strength to the support and also provides an excellent degree of thermal shock resistance because of the ability of the porous matrix to absorb thermally induced mechanical stresses which might otherwise fracture the fibers in the filter.

The support is formed of a plurality of layers of continuous ceramic oxide yarns which are laid down in spaced helical coils in a crisscrossing relationship with neighboring layers to form a plurality of diamond or quadrilateral-shaped openings having dimensions between 100 and 500 microns after firing. The openings form channels extending between the inner 22 and outer 20 surfaces of the support which follow tortuous, curved paths (see FIG. 1B). If the filter is damaged, for example by damaging the membrane layer during installation, it will quickly "self-heal" by functioning as a bulk filter and blinding with particulates in the hot gas stream. A support containing a significant number of straight radial channels will not blind as readily, resulting in failure of the filter. Forsythe, U.S. Pat. No. 5,192,597, incorporated herein by reference, describes filament winding of reticulated ceramic tubes in a preferred winding pattern. The yarns in adjacent layers of diamond-like patterns are laid down in such a manner that the yarns forming the walls of the diamonds of each layer substantially cover the diamond shaped openings of each adjacent layer. This forms a tubular structure comprising series of interconnected diamond shaped openings, each layer of which interfere with the direct flow of gas from one layer to the next.

The winding pattern described above is for the elongated central body section of the support (i.e. the generally cylindrical section of the filter between the flange and closed end). Due to the contoured closed end and flange sections of the filter, the described winding pattern is not achieved at the flange and closed end.

FIG. 2 shows two adjacent layers of yarn in a support prepared according to U.S. Pat. No. 5,192,597 (the matrix layer is not shown in this Figure) which define openings designated by " $x$ ". The size of the openings is controlled by the spacing between the yarns in each layer which is determined by the wind angle and yarn denier in addition to the amount of matrix material applied to the yarn. The spacing " $\mathrm{a}$ " between adjacent yarns is preferably controlled 60 to provide openings having dimensions " $\mathrm{a}$ " of between about 100 and 500 microns in the final support, after high temperature firing. The openings have more of a square shape near the inner surface of the support, with one of the diagonals gradually increasing in size, as winding continues, 65 to the outer surface, thereby according the opening more of a diamond shape. The dimension " $a$ " can be calculated based

\section{6}

on the yarn spacing and the amount of matrix applied to the yarn. Alternately, "a" can be measured visually in the final support. A support having the described construction and having openings in this size range will function as a bulk filter which can trap particulates within the wall of the support while maintaining a pressure drop that is insignificant relative to the pressure drop across the membrane layer.

The support may be formed by winding a ceramic oxide yarn on a suitably designed mandrel using a filament winder designed to maintain a constant winding ratio (rotational speed of the mandrel divided by the speed of the traverse arm). A constant winding ratio is necessary to maintain the proper size and distribution of channels throughout the wall. The flange section of the support is formed by using a 15 mandrel that is wider at one end, the wide end being contoured to give the desired flange geometry. Filament winding on such a mandrel produces a tube with an external flange section at the open end and a small hole at the opposite end, which is generally closed in the final support 20 with a ceramic material 26 as shown in FIG. 1E. Alternately, if it is desired that the inside wall of the support be straight as opposed to contoured at the flange section, a filament wound collar insert 24, shown in FIG. 1C and FIG. 1D, having a composition similar to that of the support and 25 having an inner diameter approximately equal to the outer diameter of the mandrel and an outer surface contoured to give the appropriate flange geometry may be used. The collar is then placed on the mandrel and the support is then wound on the combined mandrel and collar. When the support is removed from the mandrel, at least a portion of the collar remains with the support as part of the flange section, as illustrated in Example 2 below.

Field tests have demonstrated that hot-gas candle filters commonly fail at the flange section. According to the current 35 invention, the flange and the body of the support are formed as a single unit to ensure homogeneity of the support material across the entire filter and to eliminate any stresses or weak spots arising from joining materials. The shape of the flange is not critical but should be reproducible. The 40 flange should provide a good seal with the tubesheet that supports the filter in use so that no dust leakage occurs. The shape of the closed end is generally round, but various shapes are possible by suitably shaping the mandrel. The diameter of the opening at the closed end of the tube depends 45 on the diameter of the shaft that supports the mandrel.

The membrane layer is applied to the outer surface of the support, the inner surface of the support, or to both the outer and inner surface of the support. The membrane layer usually comprises an ordered arrangement of continuous 50 filament oxide ceramic yarns. The membrane layer optionally may also comprise one or more ceramic filler materials to help fill gaps or plug cracks, voids, etc. between adjacent yarns. In the alternative, the membrane layer may comprise the ceramic filler material, but no ceramic yarns. The mem55 brane layer(s) in the final filter, after heat treatment, has pore diameters of between about $0.1-50$ microns, preferably 5-25 microns. Preferably, the average pore size and size distribution is substantially invariant around the circumference and along the length of the filter.

60 For those embodiments in which the membrane layer(s) comprises yarns, the ordered arrangement of yarns in the membrane layer(s) may be formed by various methods including circular (hoop) winding, multiple yarn winding, or wrapping with yarns prearranged in two or three dimen65 sional forms such as fabric or braided materials. The membrane yarns should be arranged so as to obtain a smooth membrane surface. A smooth membrane surface is desirable 


\section{$5,902,363$}

7

because it facilitates complete removal of the filtered material during backpulse cleaning because the filter cake readily debonds from the smooth surface. If the surface is rough, the filtered cake tends to be mechanically anchored to the surface making it difficult to completely remove the cake by backpulse cleaning. The circular winding produces a smooth membrane surface.

The yarns used to form the support and membrane layer (s) preferably comprise ceramic fibers having softening points of at least about $750^{\circ} \mathrm{C}$., more preferably at least $1000^{\circ} \mathrm{C}$. The phrase "softening point" is used herein to mean both the softening point of a glass ceramic and the melting point of a crystalline ceramic. The yarns used in the membrane layer(s) may be the same as or different than the yarns used in the support.

Suitable oxide fibers include, for example, certain glass fibers such as $\mathrm{S}$ glass (high tensile strength glass containing about 24-26\% alumina $\left(\mathrm{Al}_{2} \mathrm{O}_{3}\right)$ ), "Fiber Frax" aluminasilicate fiber, and polycrystalline refractory oxide fibers containing at least about $20 \%$ by weight of alumina such as the alumina-silica fibers disclosed in U.S. Pat. No. 3,503,765 to Blaze and certain of the high alumina content fibers disclosed in U.S. Pat. No. 3,808,015 to Seufert and U.S. Pat. No. 3,853,688 to D'Ambrosio. Preferably the oxide fibers comprise between $\mathbf{2 0} \%$ and $\mathbf{8 0} \%$ by weight of aluminum oxide. Examples of commercially available aluminosilicate fibers include "Altex" (Sumitomo) and "Nextel" (3M) fibers. Fibers containing significant levels of glass-forming oxides such as $\mathrm{B}_{2} \mathrm{O}_{3}$ and $\mathrm{P}_{2} \mathrm{O}_{5}$ are not desirable because they will flux the entire structure resulting in a dense, nonporous support.

Fibers of refractory oxide precursors can also be used to form the support. After winding, the precursor fibers are converted to polycrystalline refractory oxide fibers by firing to remove volatiles, convert salts to oxides, and crystallize the fiber. The preparation of refractory oxide fibers and their precursors is disclosed in U.S. Pat. Nos. 3,808,015 and $3,853,688$.

The oxide fibers generally have diameters in the range of 0.2 to 2.0 mils $(0.005-0.05 \mathrm{~mm})$ and are used in the form of continuous yarns, preferably containing $10-2,000$ or more fibers. The fibers are preferably continuous filaments, however yarns of staple fibers can be used, especially glass. The yarns are preferably loosely twisted so that any loose or broken ends do not interfere during filament winding when the yarn is pulled through small orifices. The yarns may also be used in the form of roving. Bulked, interlaced, or textured yarns may be used. However, the yarns used in the membrane layer most preferably comprise continuous filament, untextured yarns so as to obtain a membrane layer having a smooth outer surface. Glass yarns which crystallize to form refractory oxides upon high-temperature heat treatment are preferred because they are easier to handle and less likely to break during filament winding than yarns containing crystalline ceramic fibers.

The refractory oxide matrix components of the support and membrane preferably have softening points above $1000^{\circ} \mathrm{C}$., more preferably above about $1400^{\circ} \mathrm{C}$., and most preferably above $2000^{\circ} \mathrm{C}$. Preferably the matrix comprises at least $40 \mathrm{wt} \%$ alumina.

The matrix components are generally applied to the support and membrane yarn(s) in the form of a coating composition which is then fired to form a refractory oxide matrix. The coating composition used in the support may be the same as or different than the coating composition used in the membrane. The coating composition generally com-

\section{8}

prises an aqueous solution, suspension, dispersion, slurry, emulsion, or the like which contains one or more oxide particulates or oxide precursors. Preferably the oxide particulates have a particle size of 1-20 microns, more preferably $1-10$ microns, most preferably between $1-5$ microns. Particle sizes less than 20 microns are preferred because they are readily dispersed and penetrate into voids between fibers. Slurries prepared using particle sizes less than 1 micron are generally too viscous at useful solid concentrations. Oxide particulates useful as matrix materials include alumina, zirconia, magnesia, mullite, spinel, etc. Suitable matrix precursors include water soluble salts of aluminum, magnesium, zirconium and calcium such as "Chlorhydrol®" (aluminum chlorohydrate solution sold by Reheis Chemical 15 Co.), zirconyl acetate, alumina hydrate, basic aluminum chloracetate, aluminum chloride, and magnesium acetate.

Preferably, drying control additives such as glycerol and formamide may be added to the coating composition at levels of $1-5 \mathrm{wt} \%$ based on the total weight of the coating composition. The drying control additives reduce drying stresses in the green body and also eliminate macroscopic cracks on the surface of the high-temperature fired filter. Moreover, drying stresses can be further reduced by winding the support and membrane layer(s) in an environment with 25 a relative humidity of at least about $30 \%$.

The coating composition preferably includes a ceramic oxide precursor to increase the green strength of the wound structure. These soluble oxide precursors which are useful as matrix precursors also function as binders. A preferred 30 binder is aluminum chlorhydrate, and in particular, the above-mentioned "Chlorhydrol". Preferably the coating composition includes between about $10-25$ wt \% binder, calculated based on the total solids content of the coating composition. The aluminum chlorohydrate serves to bond 35 the oxide particulates of the coating together and increases the green strength of the support. The binders are incorporated into the refractory matrix upon heat treatment.

The coating composition may be applied to the support by drawing the ceramic oxide yarn through the coating com40 position prior to winding on a mandrel. Preferably, the coating composition is uniformly distributed around the fibers of the yarn. The distribution is affected by the viscosity of the coating composition, the method of application, the density (or tightness) of the yarn bundle, the nature of the 45 yarn and the amount of the coating composition. The composition should have a viscosity that is low enough to permit flow and some penetration into voids in the yarn but high enough to adhere to the yarn bundle. When the coating composition is a particulate slurry, the solids content is 50 preferably between $50-75 \mathrm{wt} \%$ and the slurry preferably has a viscosity in the range of $100-300$ centipoise. If a coating composition containing both an oxide precursor and particulate oxide powder is used, the solids content of the slurry should be adjusted to about $60-90 \mathrm{wt} \%$ of the 55 refractory oxide matrix material derived from the oxide particulate and about $10-40$ wt $\%$ derived from the precursor. It is difficult to obtain sufficient amounts of oxidecontaining materials in the coating composition using levels of precursor greater than about $40 \mathrm{wt} \%$. The amount of 60 matrix material applied to the yarn can be controlled by pulling the yarn through a suitably sized orifice to remove excess slurry. The coating composition may be also be applied to the yarn by use of a finish roll, spraying, etc. Further, the matrix coating composition may be applied to 65 the wound filamentary membrane and support by dipping the wound support in a slurry, draining off the excess and drying. Additional dipping steps may be used if necessary to 


\section{9}

provide the desired weight of matrix relative to the weight of yarn in the support. In general, it is difficult to apply the matrix coating composition by dipping without closing a significant portion of the channels in the support, which is not desirable and results in increased backpressure.

The membrane matrix coating composition may be applied to the membrane yarn using methods similar to those described for the support. Preferably the combined weight of the matrix components of the support and membrane layers comprises about $40-70 \%$ of the final weight of the filter, more preferably about $50-60 \%$. To avoid thermal stresses, it is preferable that the support yarn has generally the same composition as any membrane yarn and the support matrix has generally the same composition as the membrane matrix. In certain applications, however, different compositions may be desirable. For the same reason, it is preferable to have a weight ratio of fiber to matrix which is essentially the same in the membrane and the support.

In one embodiment, multiple yarns are combined and wound on the support at substantially the same wind angle as that of the support to fill the underlying (or the eventual overlaying) openings in the support. This may be accomplished by feeding the separate yarns through tensioning devices, dipping in a ceramic matrix particulate slurry, and combining the yarns just prior to pulling through a larger sizing orifice than that used for single yarn ends and winding on the support if an outer surface membrane is desired, and on the mandrel if an inner surface membrane is desired. The diameter of the sizing orifice is selected as described above for hoop winding.

A membrane comprising a single filament wound layer on the support or on the inner surface of the support is generally adequate for many filtration applications. Additional layers of wound yarns may be applied to increase the thickness of the membrane layer. This usually increases the particulate collection efficiency and the back pressure of the filters.

In another embodiment, the membrane layer is formed by wrapping the support or mandrel with a ceramic fabric. The fabric is wrapped on the filter support or mandrel and a matrix slurry similar in composition to that used in the support is brushed on the fabric. The slurry wets the fabric and the support, and provides bonding to the support. Any wrinkles in the fabric are removed while still wet. Additional layers of fabric are wrapped on the support or mandrel as necessary to increase the filtration efficiency. The fabrics useful for building the membrane layer include tightly woven plain and satin weaves. It may be necessary to use a matrix slurry containing matrix particulates having a smaller particle size than the matrix particulates used to wind the support in order to improve the adhesion between the filter support and fabric membrane layer. This is because the smaller particles will more readily infiltrate the interstices in the woven fabric. In general, this method is less preferred because it is more difficult to control the amount of matrix applied to the membrane layer. In addition, it has been found that the fabric layers tend to be less strongly adhered to the support than membranes formed using the filament winding techniques described above.

In still another embodiment, the membrane is formed by 60 hoop winding. The oxide ceramic membrane yarn is coated with the membrane matrix coating composition, for example by passing through a bath containing a coating composition, followed by passing through a sizing orifice to remove excess slurry, and winding at approximately 90 degrees to 65 the axis of the mandrel. Preferably, the diameter of the sizing orifice is carefully selected to give a matrix pick-up that

\section{0}

yields similar weight ratios of fiber and matrix in the membrane and support layers. The rate of mandrel rotation relative to the rate of the movement of the transverse arm controls the spacing between adjacent yarns. FIG. 3A illustrates the cross-sectional view of a filter created in this fashion, where "a" represents the end view of a yarn in the support body, "b" represents the end view of a yarn in the hoop-wound membrane, and "c" represents the spacing between adjacent yarns in the hoop-wound membrane. In one version of this embodiment, the slurry coated membrane fiber or yarn is wound as close as possible with substantially no overlapping of yarns or intentional gaps between yarns in the membrane layer (e.g., dimension "c" in FIG. 3A equals zero). Filtering action is provided by the micro-cracks in the matrix material between adjacent yarns. Optionally, filler material may be applied to the wound membrane to fill in any unintentional gaps between adjacent yarn hoops.

In another version of this embodiment, an intentional gap is left between adjacent hoop windings of the slurry coated 20 yarn. Additional ceramic filler material, (e.g., particulates) or a precursor to a ceramic filler material, preferably in the form of a slurry, is then deposited in this gap. A membrane formed in this way is termed a "combination membrane". Preferably the gap-filling slurry should contain a suspension 25 agent to maintain a uniform consistency. The desirable viscosity will depend on the method of application chosen; low viscosities are best suited for a brushing technique, paste-like high viscosities are more appropriate when applying with a spatula. FIG. 3B illustrates the cross-sectional 30 view of a filter having a combination membrane, where "a" represents the end view of a yarn in the support body, "b" represents the end view of a yarn in the hoop-wound membrane, "c" represents the spacing between adjacent yarns in the hoop-wound membrane, and " $\mathrm{d}$ " represents the 35 filler material used to fill the spaces between adjacent yarns. This additional ceramic filler material may be of the same chemical composition as the membrane matrix material coating the membrane yarn, or it may have a different chemical composition. Typically, constituents used for gap filling are larger (e.g., 25-75 microns) than those particulates used for matrix formation (e.g., 3-5 microns). Further, the intentional spacing "c" is almost infinitely variable; it may range from substantially zero to many times the diameter of a yarn.

In yet another embodiment, because there appears to be no upper limit to the size of the gap between adjacent windings in the membrane layer, it is possible to dispense completely with the hoop wound slurry coated yarn, leaving the membrane layer to consist essentially of the ceramic 50 filler material. FIG. 3C illustrates the cross-sectional view of a filter created in this fashion, where "a" represents the end view of a yarn in the support body and "d" represents the ceramic filler material used to fill the quadrilateral-shaped openings at the surface of the support body. Again, such 55 material preferably is in the form of a slurry or solution which can be applied directly to the support layer by brushing, spraying, dip coating, etc. Also, the preferred size of the constituents making up a "filler material only" membrane layer is about $25-75$ microns in diameter.

The above discussion generally pertains to the form of the invention in which the membrane layer is applied to the outer surface of the support layer. When the membrane layer is to be applied to the interior surface of the support layer, the fabrication procedures may have to be modified. For 65 example, when a fiber or fabric is to make up the membrane layer, it may be preferred to wind or wrap such fiber or fabric over the mandrel before winding the support layer. Also, if 
11

the inner membrane layer is "filler material only" or if an additional slurry or solution is to be deposited onto a hoop-wound filament layer, it may be preferred to do so once the support layer has been formed and the mandrel has been removed. Further, it may be impractical to apply a slurry or solution to the interior of a tube by brushing or spraying. In such a case, slip casting or drain casting should achieve the desired results.

The flange section and the closed end may be reinforeed and made impervious to any gas streams by saturating with additional ceramic slurry or using a ceramic cement composition. To avoid reactions with the underlying support material and to match the thermal expansion of the support, the matrix material used in the support is preferred for this purpose. After winding and reinforcing one or both ends, the candle filter is dried at room temperature while on the mandrel until it is strong enough to handle.

After overnight drying (about 12-16 hours) at ambient temperature, the ends of the developing filter are cut off so that the mandrel may be removed. Specifically, the collar portion of the filter is sliced such that a section of the original collar remain in the flange section of the support layer (see FIG. 1D).

The developing candle filter may then be fired at temperatures below the softening point of the ceramic yarn and sufficiently above the boiling point of any volatiles, typically around $300^{\circ} \mathrm{C}$. to $800^{\circ} \mathrm{C}$, , to remove the volatiles and stabilize the filter. This is especially important when oxide precursors are used.

Closing off the tip may then be accomplished using commercial high temperature cements or by filling with a high viscosity paste (similar in composition to the matrix coating slurry) mixed with a small amount of the type of yarn used in the support structure, or by filling with thickened paste similar to the membrane filler material. The 35 solids in commercial cement should not react with the tube material to reduce the thermal stability of the filter. It is also preferable to have fired the candle filter, as described above, prior to the application of a ceramic filler material to the membrane layer(s).

An additional firing at high temperatures is then carried out, typically at $1200^{\circ}$ to $1400^{\circ} \mathrm{C}$., to form stable crystalline phases. Firing above $1450^{\circ} \mathrm{C}$. may melt some of the phases and result in a fused product which is undesirable due to reduced thermo-mechanical properties. Preferably, the heating rate during the high temperature firing does not exceed $20^{\circ} \mathrm{C}$. per minute, in order to allow any glass phases to crystallize, and may be as low as $0.1^{\circ} \mathrm{C}$. per minute. During high temperature firing glass fibers may devitrify into crystalline phases, the matrix may convert to stable crystalline phases or the crystalline phases in the fiber and matrix may react to form new stable crystalline phases. The final phase composition of the product depends on the amounts of fiber and matrix, the heating profile, soaking time at intermediate temperatures and the dwell time at the highest firing temperature. The typical crystalline phases are corundum, mullite, cordierite and cristobalite. As used herein, the term cordierite is intended to include indialite, a crystalline material having the same composition as cordierite, but a slightly disordered crystal structure. Excess cristobalite formation is undesirable since cristobalite undergoes a volume change at $200-270^{\circ} \mathrm{C}$., which contributes to poor thermal shock resistance. The final filter should contain no more than $10 \%$ by weight cristobalite. Preferably the final composition of the filter is $3-7$ parts by weight magnesia, $20-45$ parts silica 65 and 45-70 parts alumina. More preferably the final filter comprises between about $60 \%-70 \%$ alumina.

\section{2}

In a preferred embodiment, the yarn used to prepare both the support and membrane comprises glass fibers comprising 61-66\% $\mathrm{SiO}_{2}, 24-26 \% \mathrm{Al}_{2} \mathrm{O}_{3}$, and $9-10 \% \mathrm{MgO}$. A coating composition consisting essentially of alumina is 5 applied to the yarn prior to winding in an amount sufficient to provide a refractory oxide matrix comprising $40-70 \%$ of the final weight of the filter. The coating composition contains a binder comprising aluminum chlorhydrate and alumina matrix particulates having an average particle size

of $2-3$ microns. The membrane is applied to the support or mandrel by hoop winding. The as-wound filter element is heated to remove volatiles and then high temperature fired at temperatures above about $1350^{\circ} \mathrm{C}$, preferably at a temperature of about $1380^{\circ} \mathrm{C}$. During high temperature firing, the 5 glass fiber softens and a portion of the silica and magnesia in the glass combine with the alumina matrix material to form cordierite and mullite. The final filter comprises about $20-40 \%$ by weight $\mathrm{SiO}_{2}$, about $3-6 \%$ by weight $\mathrm{MgO}$ and about $50-70 \%$ by weight $\mathrm{Al}_{2} \mathrm{O}_{3}$. The final crystalline 20 composition, after heat treatment, is $25-40 \%$ cordierite, $5-15 \%$ mullite, $40-60 \%$ corundum and $0-10 \%$ cristobalite, based on the total crystalline content. Approximately $50-90$ $\mathrm{vol} \%$ of the material is crystalline with the remainder being amorphous. The formation of crystals of mullite, cordierite, 25 and corundum, each having different coefficients of thermal expansion, leads to formation of microcracking in the structure. The microcracks form along crystalline boundaries as well as within regions having only a single crystal phase. The microcracks are believed to absorb stresses caused by 30 thermal shock. After firing, the filter is stable up to $1200^{\circ} \mathrm{C}$. for extended periods of time and has excellent thermal shock resistance.

\section{EXAMPLES}

All percentages referred to herein are weight percent, unless otherwise indicated.

The filament winder used to wind the support in the Examples below had a chain-driven traverse of approximately 70 inches $(178 \mathrm{~cm})(278$ teeth of 0.5 inch $(1.27 \mathrm{~cm})$ 40 pitch passing in a narrow loop driven and supported by $\mathbf{1 1}$ tooth drive sprockets at each end). The drive ratio was set such that the spindle rotated at a speed of 50 and 10/111 revolutions for each complete rotation of the chain loop for winding of the filter support. The mandrel was a tube having 4 a length of 65 inches $(165 \mathrm{~cm})$ and an outer diameter of 1.75 inches $(4.45 \mathrm{~cm})$ with end closures at each end. One of the end closures was conical with about a 30 degree taper on each side of the cone with a 0.50 inch $(1.27 \mathrm{~cm})$ diameter drive shaft mounted at its axis. The second end closure was 0 hemispherical $(1.75$ in $(4.45 \mathrm{~cm})$ diameter) with a 0.25 inch $(0.64 \mathrm{~cm})$ drive shaft mounted at its axis. The mandrel was attached to and driven by the spindle in such a position as to be traversed along its length by the traversing yarn guide. The mandrel was attached to and driven by the spindle via 5 the 0.50 inch $(1.27 \mathrm{~cm})$ shaft and supported in a bearing at the 0.25 inch $(0.64 \mathrm{~cm})$ shaft. It was mounted parallel to the chain-driven traverse guide such that the guide traversed above the mandrel surface at a distance of about 0.75 inch $(1.91 \mathrm{~cm})$ from the surface of the mandrel and the traverse 60 stroke extended from about 0.75 inch $(1.91 \mathrm{~cm})$ past the hemispherical closure onto the 0.25 inch $(0.64 \mathrm{~cm})$ shaft and to about 0.75 inch $(1.91 \mathrm{~cm})$ past the conical closure onto the 0.5 inch $(1.27 \mathrm{~cm})$ shaft.

A plastic collar having a $7 \mathrm{~mm}$ wall thickness and a 45 65 degree edge relative to the axis of the collar was inserted on the mandrel near the conical end to form the flange on the filter support for Examples 1 and 3. 


\section{$5,902,363$}

13

For Example 2, a separate winder having a 6 inch (15.2 $\mathrm{cm})$ traverse stroke with means to adjust this stroke to contour the package ends was used to form a collar insert for the flange section of the filter. The drive ratio was set such that the spindle rotated at a speed of 4 and 11/180 revolutions for each complete rotation of the traverse cam to provide the same wind angle in the collar insert as the wind angle in the support. A mandrel comprising a short piece of 1.75 inch $(4.45 \mathrm{~cm})$ outer diameter tube was mounted on the spindle and wrapped with 2 layers of 0.002 inch $(0.005 \mathrm{~cm})$ thick "Mylar" polyester film to facilitate removal of the wound unit. The mandrel was wrapped with 90 grams of S-glass (S-2 CG150 1/2 636, available from Owens-Corning Fiberglas Corporation of Toledo, Ohio) that was coated with an aqueous A-17 alumina slurry (see Example 1 for composition of slurry) applied in such a quantity to form a unit having 50-60 wt \% ceramic from the slurry and 40-50 wt \% ceramic derived from the feed yarn after drying. The collar insert, as wound, had the form of a cylinder of approximately 1.75 inch $(4.45 \mathrm{~cm})$ inner diameter and a $3 / 8$ inch 20 $(0.95 \mathrm{~cm})$ wall thickness with the ends of the cylinder wall exhibiting a taper of approximately $45^{\circ}$. The insert was removed from its mandrel while still wet and transferred to the mandrel on the main filament winder, described above. The insert was positioned so as to leave about 57 inches (145 25 $\mathrm{cm}$ ) of the straight tube portion of the mandrel exposed between the insert edge and the junction of the tube with the hemispherical end closure.

The filter support units were wound onto the mandrels with either the collar insert or plastic collar mounted thereon. Winding was carried out with the spindle set at a rotational speed of approximately 500-520 revolutions per minute. The final (fired) support units had diamond-shaped openings on the outer surface having dimensions of about 175-250 microns.

\section{TEST METHODS}

The density and porosity of the membrane layers was determined using mercury porosimetry. Membrane samples were prepared for porosimetry measurements using either of two methods. The membrane layer can be readily debonded from the support prior to firing of the candle assembly. The debonded membrane layer is then high-temperature fired and submitted for porosimetry measurements. Alternatively, the membrane sample may be prepared by scraping away the support layer from a sample of a high-temperature fired candle assembly. The median pore size is reported in microns and the porosity is reported in volume percent. The median pore size is the value obtained at the maximum intrusion volume.

The average oxide composition was determined using X-ray Fluorescence spectroscopy. The samples and standards were fused in a lithium tetraborate flux and the X-ray emission lines for the elements of interest were measured. The results are reported as weight percent with the samples being dried at $130^{\circ} \mathrm{C}$.

Crystalline phase compositions were determined using X-ray diffraction using a Scintag Pad X theta-theta diffractometer using $\mathrm{Cu} \mathrm{K}$-alpha radiation. The following conditions were used: copper tube operated at 45 kilovolts, 40 milliamps, goniometer radius $250 \mathrm{~mm}$, beam divergence 0.24 degree, scatter slit 0.43 degrees, receiving slit $0.2 \mathrm{~mm}$, germanium solid state detector bias $1000 \mathrm{~V}$, scan speed 0.2 degrees 2-theta per minute, chopper increment 0.03 degrees 65 2-theta, scan range 3 to 112 degrees 2-theta (overnight scans), samples front packed against filter paper in a 1 inch

\section{4}

square aluminum well-type sample holder, single sample changer. The samples were wet milled in acetone for 5 minutes in a McCrone vibratory mill using corundum grinding elements and dried under a heat lamp. The percentages of crystalline phases were determined based on a mixture of standard materials with $20 \%$ fluorite as an internal standard. Standard materials used were NIST (NBS) 674 alpha alumina (corundum), Baikowski high purity cordierite (indialite) standard, Coors mullite standard, NIST (NBS) 1879 cristobalite, NIST (NBS) 1878 quartz, and Coors spinel standards. The samples themselves were not mixed with an internal standard but were normalized to $100 \%$ of the crystalline components after dividing each measured intensity by its respective reference intensity ratios. Analysis 15 lines were: indialite at 10.4, 18.2 and 29.5 degrees; mullite at 16.5 and 26.1 degrees, corundum at 25.6 and 52.6 degrees, cristobalite at 21.8 degrees (overlap corrected for indialite), and quartz at 20.8 degrees.

\section{Example 1}

This example illustrates the fabrication of a ceramic filter according to the current invention, wherein the membrane layer is applied to the outer surface of the support and is formed using a woven glass fabric.

An alumina slurry was prepared by charging 7.0 liters of water and $20.0 \mathrm{ml}$ of formic acid in a mixing vessel. Fumed alumina having an average particle size of $13-15 \mathrm{~nm}$ (manufactured and sold by Degussa Corp., Ridgefield, N.J.) $(2.0 \mathrm{~kg})$ was added slowly with stirring. The $\mathrm{pH}$ of the dispersion was adjusted to 4.0 to 4.1 using formic acid. After stabilizing at this $\mathrm{pH}$ for two hours, $11.0 \mathrm{~kg}$ of Grade A-17 alumina (average particle size 23 microns, manufactured and sold by Alcoa Industrial Chemicals Div., Bauxite, Ark.) was added in portions and stirred overnight. Glycerol was added to the slurry at a level of $3 \mathrm{wt} \%$ based on the total weight of the slurry. The solids content of the dispersion was 62-65 weight percent and the viscosity was adjusted to 140 centipoise by water addition, measured with a Brookfield viscometer (Model No. RV1) using the \#1 spindle.

A 2-ply glass yarn (150 filaments/ply) comprising $65.2 \%$ $\mathrm{SiO}_{2}, 23.8 \% \mathrm{Al}_{2} \mathrm{O}_{3}$, and $10.0 \% \mathrm{MgO}$ having a hydrophilic sizing to aid wetting by the aqueous coating composition (S glass, designation S-2 CG150 1/2 636, available from Owens45 Corning Fiberglass Corporation) was fed through a ball tensioner, passed through the alumina slurry, and pulled out through a 0.017 in diameter $(0.043 \mathrm{~cm})$ sizing orifice to remove excess slurry. The sizing orifice controlled the amount of slurry applied to the yarn so that, after drying, 50 about $50-60 \%$ by weight of ceramic in the support was from the slurry and about $40-50 \%$ by weight was derived from the yarn. The wet yarn was then passed through a guide attached to the traverse arm of the filament winding machine and wound onto the contoured mandrel described above 55 wrapped with 2 layers of 0.002 in $(0.005 \mathrm{~cm})$ "Mylar" polyester film. The winding was stopped after about $\mathbf{1 0 0 0}$ grams of yarn were wound onto the mandrel, when the support reached the desired outside diameter (approximately $60 \mathrm{~mm}$ ). After drying overnight at room temperature, the filament-wound tube was removed from the mandrel by cutting through the wound material at about the center of the raised flange section (indicating the location of the plastic collar insert) and removing the two pieces from the opposite ends of the mandrel.

The outer membrane layer was attached to the support as follows. S-2 glass fabric (plain weave, $1.5 \mathrm{oz} / \mathrm{square}$ yard) available from Burlington Glass Fabric (Altavista, Va.) was 


\section{$5,902,363$}

15

cut into pieces of length and width approximately equal to the length and circumference of the tube respectively. Each piece was wrapped on the body of the tube and an alumina slurry containing Grade A-16 alumina (manufactured and sold by Alcoa, average particle size 0.45 micron) with 55 to 60 weight percent solid content, 3 wt \% glycerol, and 100 to $120 \mathrm{cps}$ viscosity, was brushed on the fabric. The fabric was not applied to the flange and the bottom end of the tube. Any wrinkles in the fabric were removed by rubbing with a wet sponge while the fabric was still wet before adding additional layers of fabric. Two additional layers of fabric were attached in a similar manner such that the closing of the ends in each layer of fabric fell approximately 120 degrees apart in the final filter. After all fabric layers were applied, the tube was dried overnight at room temperature. It was then lowtemperature fired at $700^{\circ} \mathrm{C}$. for one hour in a muffle furnace to remove volatiles and stabilize the structure.

The flange section was reinforced and sealed by dipping one time in an alumina slurry (fumed alumina/A-17 alumina, described above) and draining off the excess. A wad of S-2 glass fibers was inserted into the hole in the bottom end of the filter and the bottom end was then dipped in the A-17 alumina slurry. After thorough drying and firing at $700^{\circ} \mathrm{C}$. for one hour, the filter was fired in a high temperature furnace. The temperature was increased to $800^{\circ} 25$ C. in about 40 minutes, held for about 20 minutes, then increased to $1300^{\circ} \mathrm{C}$. at a rate of $2^{\circ} \mathrm{C}$. minute, held for 2 hours, then heated at a rate of $1^{\circ} \mathrm{C}$. $/$ minute to $1380^{\circ} \mathrm{C}$, held for two hours and cooled to $800^{\circ} \mathrm{C}$. at a rate of $5^{\circ} \mathrm{C}$. $/$ minute, followed by unrestrained cooling of the furnace to $200^{\circ} \mathrm{C}$. The filter was then removed from the furnace and allowed to cool to room temperature in air

The membrane layer had a bulk density of $1.62 \mathrm{~g} / \mathrm{cc}$ and a volume porosity of $39 \%$ with a median pore diameter of 0.45 micron, measured by mercury porosimetry. The average oxide composition of the filter, determined by X-ray fluorescence, was $27 \%$ silica, $68 \%$ alumina and $4 \%$ magnesia. The crystalline phase composition, determined by $\mathrm{X}$-ray diffraction, was $35 \%$ cordierite (indialite), $6 \%$ mullite, $50 \%$ corundum and $9 \%$ cristobalite.

\section{Example 2}

This example illustrates the fabrication of a ceramic filter of the current invention, wherein the outer membrane layer is formed by circular winding.

A filter support was prepared in a manner similar to that described in Example 1 except that the filament-wound collar insert was used to form the flange section instead of the plastic collar. When the support element was cut through for removal from the mandrel, the wound collar was cut through as well such that a section of the original collar remained in the flange section of the support. The mandrel with the support wound thereon was immediately transferred to a specialized winder for formation of the membrane layer. 55

The outer membrane was applied to the support by circular (hoop) winding of a glass yarn (Owens-Corning S-2 CG $150 \frac{1}{2}$ 636) on the surface of the support. The filament winder used for formation of the membrane layer had a screw driven traverse, with the drive ratio set such that the 6 spindle rotated at a speed of 75 complete revolutions for each 1 inch $(2.54 \mathrm{~cm})$ travel of the traverse guide so that the yarn was placed at a spacing of 75 yarns per linear inch (30 yarns per linear $\mathrm{cm}$ ) of tube surface. Adjacent yarn windings were as close to each other as possible without overlapping. The yarn was soaked in the A-17/fumed alumina slurry, and pulled through a 0.017 in $(0.043 \mathrm{~cm})$ sizing orifice prior to

\section{6}

winding. About 60 grams of yarn were wound on the support surface to form a single layer of winding over its length. The circular winding was done across the entire length of the filter, bottom end and flange section. After overnight drying (12-16 hours) at ambient temperature, the tube was removed from the mandrel as described in Example 1. After inspection for defects, the filter unit was fired at 700 degrees C. for two hours. Then the bottom hole was then filled with a wad of S-glass yarn. The flange and bottom sections of the tube were dipped in the A-17/fumed alumina slurry, the excess drained off, and dried thoroughly. The combined support and membrane was then high-temperature fired as described in Example 1.

The membrane layer had a bulk density of $1.61 \mathrm{~g} / \mathrm{cc}$ and 15 a volume porosity of $39 \%$ with a median pore diameter of $0.43 \mu \mathrm{m}$, as measured by mercury porosimetry. The average oxide composition of the filter, determined by X-ray fluorescence, was $27 \%$ silica, $68 \%$ alumina and $4 \%$ magnesia. The crystalline phase composition, determined by $\mathrm{X}$-ray diffraction, was $33 \%$ cordierite, $8 \%$ mullite, $49 \%$ corundum and $10 \%$ cristobalite.

\section{Example 3}

This example illustrates the fabrication of a ceramic filter of the current invention, wherein the outer membrane layer is formed by multiple yarn winding.

A filter support element was prepared as described in Example 1.

30 The outer membrane layer was formed using the same filament winder as was used to form the support. Yarns from three different bobbins of S-2 CG $1501 / 2636$ glass yarn were combined and fed through a tension device, dipped in the A-17/fumed alumina slurry described in Example 1, pulled 35 through a 0.025 in $(0.64 \mathrm{~mm})$ diameter sizing orifice, and wound on the support. The same wind angle, mandrel rotation rate, and traverse arm speed used for the support was used for winding the membrane layer. The winding was continued until two layers of yarn had been wound onto the

40 mandrel so that the yarn covered the entire surface of the support. After drying overnight, the bottom end and flange sections were treated as described in Example 2. The assembly was then high temperature fired as described in Example 1.

The membrane layer had a bulk density of $1.75 \mathrm{~g} / \mathrm{ce}$ and a volume porosity of $37 \%$ with a median pore diameter of $0.64 \mu \mathrm{m}$, as measured by mercury porosimetry. The average oxide composition, determined by X-ray fluorescence, was $27 \%$ silica, $68 \%$ alumina and $4 \%$ magnesia. The crystalline phase composition, determined by X-ray diffraction, was $35 \%$ cordierite, $6 \%$ mullite, $50 \%$ corundum and $9 \%$ cristobalite.

\section{Example 4}

This example illustrates the fabrication of a ceramic filter of the current invention, wherein membrane layers are applied to both the inner and outer surfaces of the support and are formed by circular winding.

The inner membrane was formed by the circular winding of glass yarn, saturated with a ceramic particulate slurry, around a plastic-wrapped mandrel. After preparing a mandrel (as described in Example 1), a filament-wound collar insert (as described in Example 2) was positioned so as to 65 leave about 57 inches $(145 \mathrm{~cm})$ of the straight tube portion of the mandrel exposed between the insert edge and the junction of the tube with the hemispherical end closure. The 
19

alumina slurry, and pulled through a 0.017 inch $(0.043 \mathrm{~cm})$ sizing orifice prior to winding. The circular winding was done across the entire length of the filter, bottom end and flange section. During winding, a humidity level of at least 30 percent was maintained.

Heat guns were set up to dry the reinforced regions, while maintaining rotation, for at least 20 minutes. The developing filter and mandrel were then removed from the winder and placed in a vertical support rack.

After overnight drying (about 12-16 hours) at ambient 10 temperature, the ends of the developing filter were cut off so that the mandrel could be removed. The collar portion of the filter was sliced such that a section of the original collar remained in the flange section of the support layer. The developing filter was then heated from ambient to a tem- 15 perature of about $700^{\circ} \mathrm{C}$. in a muffle furnace equipped with a hydrochloric acid scrubber. After maintaining this low firing temperature for about one hour, the furnace and its contents were permitted to furnace cool.

Next, a paste for closing the tip end of the tube and for 20 filling in the gap between windings in the membrane layer was prepared. Specifically, about $980 \mathrm{~g}$ of de-ionized water was measured out in an open container. While stirring, about $20 \mathrm{~g}$ of "Superloid" ammonium alginate (Kelco Co., San Diego, Calif.) was added. Stirring of this mixture was 25 continued until a smooth-flowing solution, free from gel particles, was obtained. Then, while continuing to stir, about $330 \mathrm{~g}$ of talc (Grade MP 12-62, manufactured by Minerals Technologies) was added to the solution. When the talc had been evenly dispersed, an additional $2700 \mathrm{~g}$ of 320 grit 38 Alundum ${ }^{\circledR}$ alumina particulate (Norton-St. Gobain, Worcester, Mass., 32 microns ave. particle size) was slowly added. Mixing was continued until a smooth paste, without apparent lumps or agglommerates, was obtained.

The low fired candle filter was slid back onto a mandrel 35 and put back onto the winder. The mandrel was rotated at approximately 100 RPM while the particulate paste was applied to the surface of the filter with a plastic spatula until the entire surface was covered. Sufficient pressure and "drag" were then applied with a clean spatula to remove most of the excess material. A cross-sectional schematic view of the tube wall is illustrated in FIG. 3B.

After removing the developing candle filter from the mandrel once again, the $1 / 4$ inch $(6 \mathrm{~mm})$ diameter opening in the tip of the candle was filled with the above-identified paste. After overnight drying, a 1.25 inch (32 mm) diameter, 4 inch $(102 \mathrm{~mm})$ long, 40-watt illuminated light bulb was inserted into the open end of the filter. All room lights were extinguished and the surface of the filter was examined. In any location where there were bright points of light ("pin holes") additional particulate paste was applied.

The candle filter was then high temperature fired as follows. The candle filter was placed into an air atmosphere furnace at about ambient (e.g., about $20^{\circ}$ C.) temperature. The furnace temperature was increased to about $800^{\circ} \mathrm{C}$. in about 40 minutes, held for about 1 hour, then increased to about $1300^{\circ} \mathrm{C}$. at a rate of about 20 per minute, held for about 2 hours, then increased to about $1380^{\circ} \mathrm{C}$. at a rate of about $1^{\circ} \mathrm{C}$. per minute, held for about 2 hours, cooled to about $800^{\circ} \mathrm{C}$. at a rate of about $5^{\circ} \mathrm{C}$. per minute, and finally furnace cooled to about $200^{\circ} \mathrm{C}$. The furnace was then opened and its contents permitted to cool naturally to ambient temperature.

\section{Example 7}

A ceramic hot gas filter was produced substantially in accordance with Example 5 except that no circularly wound

\section{0}

filaments or yarns made up the membrane layer. The slurry for the membrane layer was applied by brush, and excess particulates were gently rubbed off of the filter tube. A cross-sectional schematic view of the tube wall is illustrated in FIG. 3C.

What is claimed is:

1. A ceramic hot gas filter, comprising:

a porous elongated filter support, said support having an outer surface, an opening at one end into a hollow interior defined in part by an inner surface, a closed end opposite said open end, and an external flange integral with said open end, said support being formed of a plurality of layers of oxide ceramic yarn, each layer being arranged in a crisscrossing relationship with neighboring layers to form a plurality of quadrilateralshaped openings, said yarn being coated with first oxide ceramic material, said first oxide ceramic material providing, upon heat treatment, a porous refractory oxide support matrix; and

a porous membrane layer contacting the outer surface or inner surface of said support, said membrane layer being less porous than said support and comprising (1) at least one circularly wound continuous filament oxide ceramic yarn, adjacent windings of said ceramic yarn defining a gap therebetween, said yarn being coated with a second oxide ceramic material, and (2) at least one ceramic filler material disposed in said gap and substantially uniformly distributed therein.

2. The filter of claim 1, wherein said at least one ceramic filler material comprises a particulate oxide ceramic material.

3. The filter of claim $\mathbf{1}$, wherein said at least one ceramic filler material comprises a form selected from the group consisting of powders, particulates, whiskers, chopped fibers, platelets, flakes, spheres, tubules and pellets.

4. The filter of claim 1 , wherein said filter has a crystalline composition of about $25-40 \%$ cordierite, $5-15 \%$ mullite, $40-60 \%$ corundum, and $0-10 \%$ cristobalite, based on the total crystalline content of the filter.

5. The filter of claim 1, wherein said quadrilateral-shaped openings have dimensions of about 100 to about 500 40 microns after heat treatment.

6. The filter of claim 1, wherein said membrane layer defines pores having diameters of about 0.1 to about 50 microns.

7. The filter of claim 1, wherein said membrane layer 5 defines pores having diameters of about 5 to about 25 microns.

8. The filter of claim 7, wherein an average size and size distribution of said pores is substantially invariant around a circumference and along a longitudinal extent of said mem50 brane layer.

9. The filter of claim 1 , wherein said second oxide ceramic material provides, upon heat treatment, a porous refractory oxide membrane matrix.

10. The filter of claim 9, wherein between about 40 to 55 about 70 percent of the total weight of said filter is from the combined weight of said support matrix and said membrane matrix.

11. The filter of claim 1, wherein said support yarn has generally the same composition as said continuous filament 60 membrane yarn and wherein said first oxide ceramic material has generally the same composition as said second oxide ceramic material.

12. The filter of claim 1, wherein said first and second oxide ceramic materials each comprise $\mathrm{Al}_{2} \mathrm{O}_{3}$.

65 13. The filter of claim 1 , wherein said porous membrane layer contacts both the outer surface and the inner surface of said porous elongated filter support. 


\section{1}

14. A ceramic hot gas filter, comprising:

(a) a porous elongated filter support, said support having an outer surface, an opening at one end into a hollow interior defined in part by an inner surface, and a closed end opposite said open end, said support being formed of a plurality of layers of oxide ceramic yarn, each layer being arranged in a crisscrossing relationship with neighboring layers to form a plurality of quadrilateralshaped openings, said yarn being coated with first oxide ceramic material, said first oxide ceramic mate- 10 rial providing, upon heat treatment, a porous refractory oxide support matrix; and

(b) a porous membrane layer contacting at least one of the outer surface or inner surface of said support, said membrane layer being less porous than said support ${ }^{15}$ and comprising (1) at least one hoop wound continuous filament oxide ceramic yarn, adjacent windings of said ceramic yarn defining a gap therebetween, said yarn being coated with a second oxide ceramic material, said second oxide ceramic material providing, upon heat 20 treatment, a porous refractory oxide membrane matrix, and (2) at least one ceramic filler material deposited in said gap and substantially uniformly distributed therein.

15. The filter of claim 14, wherein said support yarn and 25 said continuous filament membrane yarn each comprise at least 20 weight percent alumina and have a softening point above about $750^{\circ} \mathrm{C}$

16. The filter of claim 14 , wherein a weight ratio of said yarn to said matrix is essentially the same in said membrane 30 layer as in said support.

17. The filter of claim 3 , wherein said first and said second oxide ceramic materials comprise particulate, and further

\section{2}

wherein said form of said at least one ceramic filler material has a size which is larger than a size of said particulate.

18. The filter of claim 14, wherein said at least one filler material comprises bodies having a size of about 25 to 75 microns.

19. The filter of claim 14 , wherein said membrane layer contacts the outer surface of said support, and is made by a method comprising:

(a) fabricating said elongated porous filter support by coating a ceramic oxide support yarn with a first coating composition, winding said coated ceramic oxide support yarn onto a mandrel to form a plurality of layers of said coated support yarn, each layer being arranged in a crisscrossing relationship with neighboring layers to form a plurality of quadrilateral-shaped openings, said first coating composition providing, upon heat treatment, a porous refractory oxide support matrix;

(b) coating at least one continuous filament oxide membrane yarn with a second coating composition, and winding said coated yarn onto said filter support, said winding being conducted so as to leave a gap between adjacent windings of said coated yarn;

(c) depositing a slurry or paste comprising a suspending agent and at least one ceramic filler material into said gap;

(d) drying said paste to form said membrane layer; and

(e) firing said support and membrane layers. 
Appendix 2

\section{DLC Raw Materials Specification Form}


SOP DLC-8.2

Page 1 of 8

Effective Date:

July 1,1996

SUBJECT: $\quad$ Material Specifications

Rev. A EO: 4

PURPOSE: To document the procedure to develop and update Material Specifications for Essential Materials to be purchased

EXPLANATION OF CHANGE: ORIGINAL ISSUE

AUTHORIZED BY:*

Manufacturing Manager (DLC) Gene Mathis/s/

Date: $\underline{6 / 10 / 96}$

Business Manager (DLC)

Gary Knox/s/

Date: $\underline{6 / 14 / 96}$

Technical Manager (DLC)

Aspi Patel/s/

Date: $\underline{6 / 5 / 96}$

Purchasing Mgr (Lanxide Corp)

Debbie Facciolo/s/

Date: $\underline{6 / 11 / 96}$

* Electronic EO signatures on file in the TPN Fileserver; paper EO signatures on file with the EO Coordinator

DU PONT LANXIDE COMPOSITES INC.

1300 MARROWS ROAD

NEWARK, DELAWARE 19714 
1. SCOPE

\subsection{Purpose}

1.1.1 This document establishes the content and administration of Material Specifications for Du Pont Lanxide Composites Inc. (DLC).

\subsection{Applicability}

1.2.1 This procedure applies to all goods and services that are Essential Materials for DLC products sold to customers. This SOP does not apply to materials bought for internallyfunded experiments and conceptual development.

\subsection{Terminology}

1.3.1 An Essential Material is any material (including tooling) that directly impacts product quality and that cannot be changed without affecting plant performance, customeruse requirements, or product quality.
1.3.2 Quality Manual Section 3.0 (Terms and Definitions) contains definitions of other terms used in this document.

\subsection{Auditing}
1.4.1 The Management Representative will audit this SOP at least once a year.

\section{REFERENCES}

2.1 Quality Manual Sections 3.0 (Terms and Definitions) and 8.0 (Quality in Procurement),

2.2 SOP DLC-7.1, Document Control

2.3 SOP DLC-8.1, Purchase of Goods and Services

2.4 SOP DLC-11.1, Material Receiving Inspection 


\section{RESPONSIBILITIES}

3.1 The Project Engineer (or equivalent responsibility) is responsible to develop a Material Specification (MS) for each new Essential Material to be bought and used to make a product sold to a customer.

3.2 The Project Engineer is also responsible to make sure the MS is kept up-to-date during the production life of the product. As part of the set-up for a new or revised material, the Project Engineer also completes a new Material Receipt Inspection Log in the TPN Fileserver (SOP DLC-11.1, Material Receiving Inspection).

3.3 The requisitioner of an Essential Material will:

- print and attach a copy of the MS to each "Purchase Requisition/Blanket Order Release" form submitted to Lanxide Purchasing to buy the respective Essential

- attach a copy of the Material Safety Data Sheet (MSDS) to a Purchase Order whenever the MS references an MSDS (if DLC does not have an MSDS on file, the requisitioner requests one from the supplier)

- list such items as Certificates of Analysis or Conformance as deliverable items on the Purchase Requisition.

\section{PROCEDURE}

4.1 Attachment 1 is a template for the contents of each MS. The MS will be generated and kept in the "Material Specification" database on the TPN Fileserver.

4.2 Attachment 2 lists the Quality Assurance Codes which print their respective statements on a printed MS when specified in the database.

4.3 The Engineering Order (E.O.) form is the mechanism to approve new or revised MSs (ref.: SOP DLC-7.1, Document Control)

4.4 The Quality Plan for each Control Level 1 product will specify Essential Materials and will reference the MS numbers. 


\section{Attachment 1}

Material Specification (MS) Content

1. Material

Application

Chemical Formula: (if applicable)

MS Number and Revision No.

DLC Part No

DuPont MS replaced (if applicable)

2. Approved Supplier(s)

Addresses

Supplier's phone number

Supplier's Part No:

3. Physical Specifications:

Dimensions:

Weights:

Workmanship Standards:

Materials:

Material Lot Numbers:

Drawing Numbers

Other (Thermal specifications, Conductivity, etc.):

4. Yarn/Fabric/Prepreg Specifications

Property Units Aim Lower Limit Upper Limit

Other Specifications

5. Chemical Specifications: (if applicable)

Property Units Aim Lower Limit Upper Limit Test Method

Appearance:

Chemical Identification Method:

Other:

6. Packaging:

Container Type:

Container Material:

Container Size:

Container Labeling:

Other Packaging Info: 
7. Acceptance/Rejection

Lot Size:

Inspection/Test

Inspection/Test Method

Decision Criteria (“Accept If”):

8. Safety, Health, and Environmental Information:

Hazardous Material: Yes No

MSDS No. Rev Date:

Is this, or does this contain, an ozone-depleting substance: Yes_No

DOT Reg.: (if applicable)

9. Handling, Storage, Preservation and Disposal Information:

Expiration Date, if any

Handling Requirements:

Storage Requirements:

Disposal Requirements:

Shipping Requirements:

10. Quality Assurance Requirements:

(Inserts appropriate paragraph to match QA codes entered. Nothing will be printed if Code " 00 "” is entered-a "required entry" field))

Key Characteristics (if any - to accompany Code \#15)

Other Quality Requirements

11. Pertinent Information

Applicable Documentation

12. Other Information: (e.g., minimum order quantity...)

13. Revision History

Revision Date:

MS Change

EO Number:

Author: 
Attachment 2

Quality Assurance Codes

Code

Description

$00 \quad$ No Extra Quality Systems Requirements

(None printed—the "default" required entry)

$01 \quad$ Certificate of Conformance

The supplier shall submit a Certificate of Conformance with each shipment that is signed by an authorized supplier's representative and states that the materials supplied to Du Pont Lanxide Composites are in conformance with applicable requirements of the contract, drawings, and specifications and that supporting documentation is on file and will be made available to Du Pont Lanxide Composites, Du Pont Lanxide Composites' Customer, or Government representatives upon request. The Certificate of Conformance must include: Du Pont Lanxide Composites part number, purchase order number, revision level, quantity, and any exceptions to specification or purchase requisition requirements.

02 Certificate of Analysis

The supplier shall submit a Certificate of Analysis with each supplier's material lot in each shipment that is signed by an authorized supplier's representative and states that each property value contained was the result of a valid laboratory test or analysis. The Certificate of Analysis must include: Du Pont Lanxide Composites' part number, purchase order number revision level, manufacturer's lot number, manufacturer's lot production date, analyses and test values, corresponding analysis or test method number (including reference to ASTM or equivalent standard method).

03 Receiving Inspection at Du Pont Lanxide Composites

Items purchased under this purchase order are subject to incoming inspection and final acceptance at the Du Pont Lanxide Composites facility named on the purchase order.

04 Du Pont Lanxide Composites Inspection at the Supplier's Facility

Du Pont Lanxide Composites source inspection is required before shipment of items from your facility. Notify Lanxide Corporation buyer (agent for Du Pont Lanxide Composites) at least three (3) working days before the scheduled date of shipment from your facility.

05 Government Inspection at the Supplier's Facility

Government inspection is required before the shipment of this item. Upon receipt of this purchase order, promptly notify the Government Representative who normally services your plant to plan appropriately for Government inspection. If not, notify the nearest Defense Supply Agency Inspection office in your area.

06 Customer Inspection at the Supplier's Facility

Inspection by Du Pont Lanxide Composites' is required before the shipment of this item. Notify Lanxide Corporation buyer (agent for Du Pont Lanxide Composites) at least five (5) working days before the scheduled date of shipment from your facility.

07 Dimensional Inspection Report

Dimensional inspection data for all drawing attributes shall be included in an Inspection Report on all items delivered under this purchase order. This report shall reference part number, revision level, serial number (if applicable) and the purchase order number. This report will be shipped with the material, else the material will be rejected by receiving inspection and may be returned at the supplier's expense.

08 Special Process Certification 
The supplier shall have records of any special process(es) he is qualified/certified to perform available for review by Du Pont Lanxide Composites personnel. Examples of special processes are: cleaning, welding, plating, soldering, and non-destructive testing. The supplier shall identify any sub-tier suppliers that perform special processes and supply this information to $\mathrm{Du}$ Pont Lanxide Composites with each shipment. Approval of Inspection Procedures

The supplier shall provide a detailed inspection procedure that describes the inspections to be performed, where they occur in the manufacturing cycle, and the equipment to be used. These procedures are subject to Du Pont Lanxide Composites' approval before starting actual work. Approval of Test Procedures

The supplier shall provide a detailed test procedure that describes the tests to be performed, test methods, test equipment and environment, and the sequence of testing and test data requirements. These procedures are subject to Du Pont Lanxide Composites' approval before starting actual work.

11 Customer Witness

A representative of Du Pont Lanxide Composites' customer may witness any inspection or test without affecting Du Pont Lanxide Composites' exclusive right to give direction to the supplier or to accept or reject any procedure, test data, or item.

12 Government Witness

A Government representative may witness any inspection or test without affecting Du Pont Lanxide Composites' exclusive right to give direction to the supplier or to accept or reject any procedure, test data, or item.

13 Written Approval for Changes

The supplier shall notify Du Pont Lanxide Composites of any changes in design, fabrication methods, or processes and obtain Du Pont Lanxide Composites' written approval before making the changes.

14 Reporting of Test Data

All test data shall be reported in the correct format: either 1) "variables" format when the test method produces data on a continuous numeric scale, or 2) "attribute" format for such counted data and defects or "pass/fail". In addition to the lot average data, the sample standard deviation(s) and Sample size are to be reported for each characteristic. If multiple test replicates are run on product samples from the same lot, portion average will be used for the lot average (use as single data point) and not each individual replicate.

15 Key Characteristics

Key Characteristics (those specified in the Purchase Order or Material Specifications) of product supplied must have a minimum process capability, Cpk, of 1.0 with a $90 \%$ confidence level (this translates into Cpk of 1.30 minimum for a sample size of 20 data points to a Cpk of 1.07 for sample sizes of 250 data points). This process capability shall be substantiated by process capability calculations on the certifications supplied with the shipment.

16 Material Safety Data Sheet to be Provided

The supplier shall include a copy of the latest Material Safety Data Sheet (MSDS) with the first shipment of each item in this purchase order.

17 Proof of Statistical Control

Supplier shall provide proof of statistical control of key properties. The proof will be in the form of property histograms and control charts for the lot(s) shipped. 


\section{Appendix 3}

This appendix contains a copy of the Summary Report of work performed by Westinghouse Electric Corporation, Science and Technology Center, under a subcontract of this program. 


\title{
ADVANCED HOT GAS FILTER DEVELOPMENT SUMMARY REPORT
}

\author{
M. A. Alvin \\ March 31, 1998
}

\author{
By \\ Westinghouse Electric Corporation \\ Science and Technology Center \\ 1310 Beulah Road \\ Pittsburgh, PA 15235-5098 \\ Under \\ Westinghouse Reference No. WL-1 3059-CE \\ DuPont/DOE FETC Contract No. DE-AC2I-94MC31214A
}

\author{
For \\ DuPont Lanxide Composites \\ 1300 Marrow Road \\ P. 0. Box 6077 \\ Newark, DE 19714
}




\title{
ADVANCED HOT GAS FILTER DEVELOPMENT
}

\section{SUMMARY REPORT}

\author{
M. A Alvin \\ March 31, 1998
}

\begin{abstract}
During the past five years, the filament wound DuPont PRD-66 filter element has undergone considerable development to improve the structural integrity of the outer membrane, and to produce a nearly complete barrier vs. bulk filter element. Additional improvements have included the incorporation of a strengthened, integral flange and reinforced end cap area, and achievement of acceptable gas flow resistance through the as-manufactured filter body.

DuPont PRD-66 filters were installed and operated in the Westinghouse Advanced Particulate Filtration unit at the American Electric Power pressurized fluidized-bed combustion test facility in Brilliant, OH, in 1994 and 1995, and at the Foster Wheeler pressurized circulating fluidized-bed combustion test facility in Karhula, Finland, in 1997. Both field test operations, as well as bench-scale qualification testing conducted in Westinghouse's pressurized fluidized-bed combustion simulator test facility in Pittsburgh, PA, have identified several life limiting issues that warrant continued development prior to commercial use of the filament wound PRD-66 candle. Additional efforts remain to be focused on the development and production of a dual membrane, barrier candle filter; further strengthening of the flange; and incorporation of a chip resistant outer surface. This report provides a summary of the efforts conducted at Westinghouse which have supported the development, manufacture, and field test operation of the DuPont PRD-66 candle filters.
\end{abstract}

\section{Introduction}

Two tasks were conducted by Westinghouse in support of DuPont's DOE/FBTC program entitled "Advanced Hot Gas Filter Development" (Contract No. DE-AC21-94MC3 1214A). These included:

Task 2- Test Plan Definition

Task 3- Development, Qualification, and Testing of Hot Gas Filters. 
Initially Task 3 was identified to include:

Task 3.1 - Material Qualification

Task 3.2- Corrosion Testing

Task 3.3 - High Temperature, High Pressure (HTHP) Filter Testing.

Due to budget constraints incurred by DuPont, Task 3.2 was eliminated from Westinghouse's workscope. In the following sections, a summary of the results obtained at Westinghouse between February 9, 1995 and March31, 1998 for conduct of Task 2, Task 3.1, and Task 3.3 is provided.

\section{Program Overview}

On January 20, 1994, the dimensional tolerances and filtration characteristics that are required for retrofit of porous ceramic candle filters into Westinghouse's Advanced Particulate Filtration (APE) systems were provided to the DuPont Lanxide Corporation $(\text { DLC })^{1}$. During 1994, filter elements were fabricated by DLC, and were delivered for use in the Westinghouse APE slipstream test facility that was operated at the American Electric Power (AEP) pressurized fluidized-bed combustion (PFBC) Tidd Demonstration plant in Brilliant, Ohio. The Westinghouse APF system at AEP consisted of three filter clusters (i.e., nine filter arrays) which housed 384, $1.5 \mathrm{~m}$ filter elements.

Testing of three, $1.5 \mathrm{~m}$, DLC PRD-66 filament wound candles in the PFBC environment was initiated in July 1994, and continued for a period of 1705 hours [1]. At the conclusion of testing in October 1994, the filter vessel was slow cooled and inspected. Posttest inspection indicated that all three filters elements remained intact.

Additional 1.5-m PRD-66 filter elements were fabricated for inclusion in Test Segment 5 at AEP (January through March 1995). Twenty-two PRD-66 candle filters were installed in the Westinghouse APF system, filling an entire top array. After 232 hours of operation, sections of the PRD-66 matrix were identified in the ash hopper discharge, implying that failure of an element or elements had occurred. Testing continued, and after 775 hours of operation, additional sections of the PRD-66 filter matrix were found in the ash hopper discharge.

At the conclusion of 1110 hours of operation in Test Segment 5, the filter vessel was slow cooled and inspected. Only two ERD-66 filter elements remained intact, four had suffered either mid-body fracture or failure at a location that was $\sim 3 / 4$ below the flange, and sixteen filters had fractured at the base of the flange. The outer surface of the intact and fractured filters was generally "ash free", particularly along the portion of the body that was adjacent to the plenum support pipe, and to approximately mid-way down the length of each filter element. Alternately a 1-2 mm ash deposit remained along the outer surface of the PRD-66 candles, primarily near the bottom end cap. Surface "divot-like" formations resulted in lines which ran parallel down both sides of the remaining intact and fractured filter elements. Localized "divoting" was also observed below the gasket sleeve, which was installed around the filter flange, as well as in alternate, isolated areas along the filter body.

\footnotetext{
${ }^{1}$ Proprietary Westinghouse filter specifications served in part fulfill Task 2- Test Plan Definition.
} 
The mechanisms leading to divoting and mid-body failure of the FRD-66 filter elements in Test Segment 5 were considered to be primarily related to delamination areas that were present within the wall of the filament wound matrix (i.e., uneven winding and/or localized drying or positioning of the elements during manufacturing of the elements). Posttest inspection indicated that ash and sorbent fines were present within the $7 \mathrm{~mm}$ PRD-66 filter wall. These were expected to have resulted from penetration of submicron fines through the PRD-66 outer membrane, or were back pulsed into the matrix after failure of an alternate candle(s). PFBC ash which had been shown by Westinghouse to have a high thermal coefficient of expansion in comparison to the ceramic filter matrix, may have induced localized internal failure within the filter wall during the plant shutdown and startup cycles in Test Segment 5. Mid-body failure of the element conceivably resulted once the filter wall had sufficiently weakened or thinned after "divoting" had occurred. Failure at the base of the PRD-66 filter flange was attributed to the low load bearing capability of the filter flange to support the thermal expansion loads applied by the ash, once fines became "wedged" in between the outer surface of the filter element and the metal holder.

In Task 2, Westinghouse recommended that

- The flange be densified and/or strengthened

- Modifications be made to the membrane to prevent fines infiltration into subsurface layers. In this manner, accumulated ash fines would not lead to fracture of the filament winding pattern during system startup and cooldown (i.e., higher thermal coefficient of expansion of the ash relative to the ceramic filter matrix).

- Modifications be made to the winding pattern to prevent localized internal delamination areas within the filter matrix,

in an attempt to mitigate failure of the PRD-66 filter element during continued process operation.

As a result, during conduct of the originally proposed contract with DOE/FETC, DLC supplied six, 1.5 m, PRD-66 candle filters to Westinghouse on February 28, 1995. Production modifications which had been made by. DLC included:

- $\quad$ Strengthening of the flange and end cap(2 Standard or baseline filter elements identified as D-337 and D-338)

- $\quad$ Strengthening of the flange and end cap, and providing a higher permeability outer surface (o.d.) membrane (2 Improved membrane filter elements identified as D-325 and D-331)

- $\quad$ Strengthening of the flange and end, providing a higher permeability o.d. membrane, as well as an inner surface (i.d.) membrane (2 Improved dual membrane filter elements identified as D-328 and D330). ${ }^{2}$

Westinghouse initially performed room temperature permeability measurements on the six modified PRD-66 filter elements to confirm DLC's measurements (Task 3.1). One filter type

\footnotetext{
${ }^{2}$ Fabrication of the dual membrane candle was recommended by Westinghouse as a result of ash penetration along the i.d. surface of intact fitter elements (i.e., AEP Test Segments 1-3) after failure. of alternate candles had occurred within the filter array during process operation. Westinghouse patent pending.
} 
of each element was then returned to DLC and sectioned. Sections were returned to Westinghouse for characterization of fines penetration into the matrix, as well as permeability measurements (Task 3.1). Following this effort, one element of each filter type was subjected to high temperature, high pressure (HTHP), simulated pressurized fluidized-bed combustion (PFBC) testing at the Westinghouse test facilities in Pittsburgh, PA (Task 3.3). After two hours of simulated PFBC exposure, and cooldown of the test facility, debonding of the outer membrane was evident. As a result continued HTHP testing was terminated, and DLC undertook an extensive effort to reformulate the manufacture and application of the membrane along the o.d. surface of the PRD-66 filter elements.

In 1997, DLC provided Westinghouse with newly formulated filter elements for qualification testing under simulated PFBC test conditions in Task 3.1. The viability and performance of the filter elements during qualification testing in Pittsburgh, PA, served as the basis for acceptance or rejection of elements for possible inclusion within Westinghouse's APF array which was installed at the Foster Wheeler pressurized circulating fluidized-bed combustion (PCFBC) test facility in Karhula, Finland. Twelve candles were subsequently manufactured and shipped directly to Karhula, Finland. After initial inspection, seven elements were identified for installation and operation in the PCFBC environment.

\section{Development, Qualification, and Testing of Hot Gas Filters}

\section{Material Qualification}

\section{Candle Filter Permeability Measurements Task 3.1)}

Westinghouse specifications for an initial pressure drop across an as-manufactured $1.5-\mathrm{m}$ candle filter is $6+/-2 \mathrm{mbar}$ at $52 \mathrm{~m}^{3} / \mathrm{hr} /$ candle at $70^{\circ} \mathrm{F}$ air $(2.41+/-0.8 \mathrm{in}-\mathrm{wg}$ at $30.6 \mathrm{scfm}$ at $70^{0} \mathrm{~F}$ air). With an outer filtration surface area of $2.76 \mathrm{ft}^{2} /$ candle filter, and a flow of 30.6 scfm, a face velocity of 11.1 fpm results.

Initial room temperature gas flow resistance measurements were conducted on the following filter elements:

- Standard or baseline candles identified as D-337 and D-338 (Strengthened flange and end cap candles)

- Improved membrane candles identified as D-325 and D-33 1 (Strengthened flange and end cap candles with a higher permeability o.d. membrane)

- Improved dual membrane candles identified as D-328 and D-330 (Strengthened flange and end candles with a higher permeability outer surface membrane, and an inner membrane).

As shown in Figure 1, relative homogeneity resulted for the standard PRD-66 candle filters which had undergone flange and end cap strengthening or densification (i.e., D-337 and D-338). Extrapolating from the gas flow resistance measurements presented in Figure 1, the pressure drop across the standard filter elements at a face velocity of $11.1 \mathrm{fpm}$ ranged between 3 and 3.4 in-wg (i.e., 7.5-8.5 mbar). Based on the room temperature gas flow resistance measurements, the standard PRD-66 candles were considered to be within the Westinghouse pressure drop specifications for as-manufactured candle filter elements. 


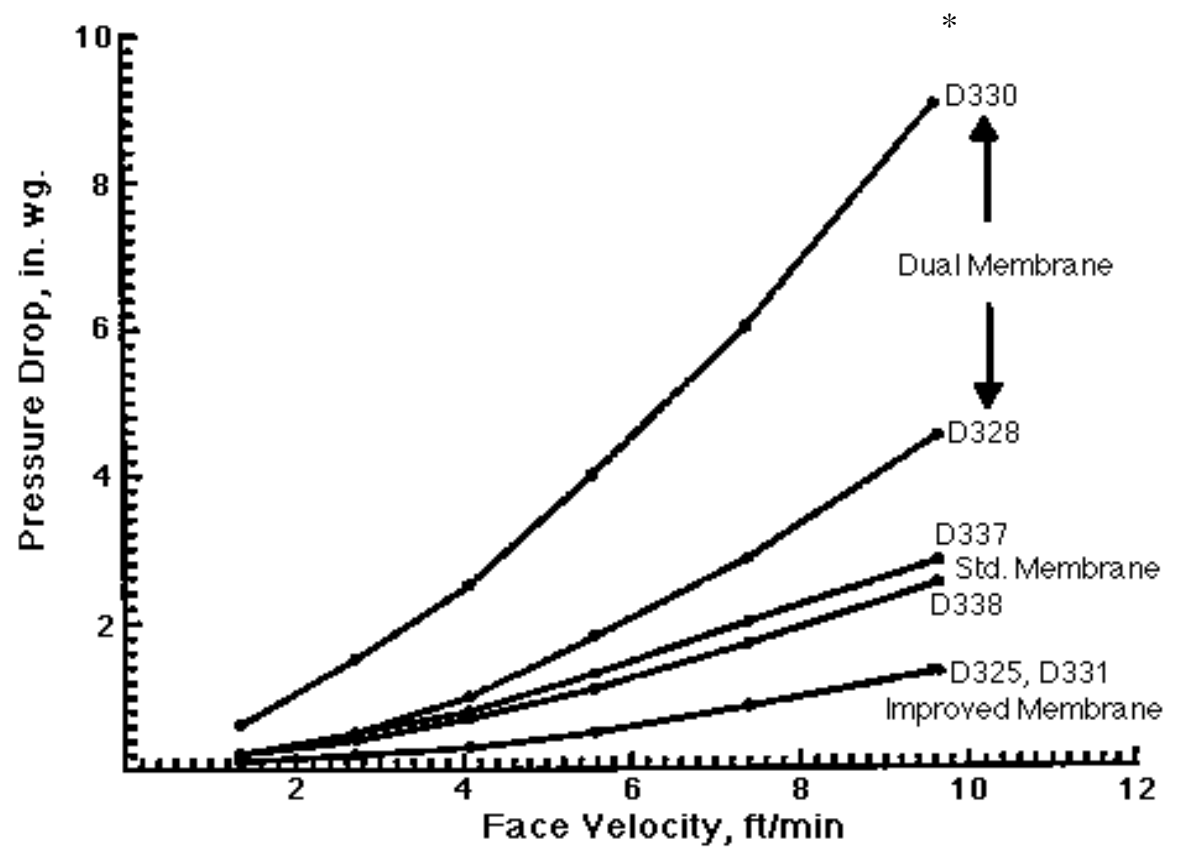

* Westinghouse As-Manufactured Specifications

Figure 1 - Room temperature gas flow resistance measurements 
With respect 10 candles that had been manufactured with an improved membrane, as well as a strengthened or densified flange and end cap (i.e., D-325 and D-331), a lower gas flow resistance resulted. As shown in Figure 1, the gas flow resistance through these elements was quite reproducible. For the improved membrane filters, the pressure drop across the candle at a face velocity of $11.1 \mathrm{fpm}$ was 1.6 in-wg (i.e., 4 mbar). This was considered to be acceptable in view of the Westinghouse as-manufactured filter element pressure drop specifications.

When the improved membrane was applied to the outside surface of the PRD-66 filament wound filter element, and an internal membrane was also applied to the i.d. surface of the filter wall, the gas flow resistance across the filer matrix increased. As shown in Figure 1 , a relatively wide range in gas flow resistance resulted between the two as-manufactured, dual membrane candle filters (i.e., D-328 and D-330). Based on the extrapolated gas flow resistance shown in Figure 1, the pressure drop across the dual membrane candles ranged between 5.6 and 11.0 in-wg (i.e., 14-27.4 mbar) for a gas face velocity of $11.1 \mathrm{fpm}$, which exceeded the Westinghouse pressure drop specifications for as-manufactured candle filters.

Based on these results, Westinghouse recommended:

- Establishing reproducibility in the manufacturing process for production of the dual membrane filter elements

- $\quad$ Further reduction of the gas flow resistance through the as-manufactured dual membrane candle filters while maintaining bulk material strength.

\section{Coupon Gas Flow Resistance and Particle Collection (Task 3.1)}

Table 1 provides a summary of the room temperature gas flow resistance measurements for twelve cylindrical PRD-66 filter samples that were supplied to Westinghouse by DLC on April 25, 1995 (i.e., D-35813, D-358C, D-358G, D-358H, D-358L, D-358M, D-359B, D-359C, D-359G, D-359H, D-359L, and D-359M). The higher gas flow resistance of samples that were designated as D-358 was supported by the visibly tighter filament winding pattern along the inner surface of the cylinders. The visibly tighter i.d. winding indicated that this series of cylinders had been manufactured with a dual membrane. In contrast, the lower gas flow resistance observed for the D-359 test sample series, as well as the open diamond weave, indicated that these samples were manufactured with only a single outer surface membrane.

The room temperature gas flow resistance of the D-359 single membrane PRD-66 cylinders was determined to be $0.51+/-0.08 \mathrm{in}-\mathrm{wg} / \mathrm{fpm}$ which indicated the relative uniformity of the six samples that were removed from various locations along the length of a single candle filter body. The room temperature gas flow resistance of the dual membrane D358 PRD-66 cylinders was determined to be $1.01+/-0.20 \mathrm{in}-\mathrm{wg} / \mathrm{fpm}$. The greater scatter in the gas flow resistance measurements for the dual membrane samples tended to indicate a reduction in production homogeneity along the length of the $1.5 \mathrm{~m}$ candle filter.

As shown in Table 1, four sections out of six of the D-358 cylinder series were within the Westinghouse gas flow resistance specifications (i.e., $<1$ in-wg/fpm), while two exceeded the as-manufactured gas flow resistance specifications. The wide range in gas flow resistance may be expected to possibly cause uneven dust cake removal. Perhaps the manner in which the membrane was applied (i.e., wetter yarn applied in one area versus another; variation in yarn 
TABLE 1

\section{GAS FLOW RESISTANCE MEASUREMENTS FOR THE IMPROVED o.d. AND i.d./o.d. MEMBRANE-COATED CYLINDERS}

$\begin{array}{ccccc}\begin{array}{c}\text { Filter } \\ \text { Identification }\end{array} & \begin{array}{l}\text { System } \\ \text { Pressure, } \\ \text { Number }\end{array} & \begin{array}{l}\text { Velocity, } \\ \text { fsig }\end{array} & \begin{array}{c}\text { Pressure } \\ \text { Drop, } \\ \text { in-wg }\end{array} & \begin{array}{c}\text { Gas Flow } \\ \text { Resistance, } \\ \text { in-wg/fmm }\end{array} \\ \text { D-358B } & 8.5 & 12.29 & 16.0 & 1.30 \\ \text { D-358C } & 8.3 & 12.24 & 12.0 & 0.98 \\ \text { D-3580 } & 5.7 & 11.51 & 10.0 & 0.87 \\ \text { D-358H } & 7.8 & 12.10 & 12.0 & 0.99 \\ \text { D-358L } & 5.7 & 11.51 & 8.5 & 0.74 \\ \text { D-358M } & 5.8 & 11.54 & 13.5 & 1.17 \\ & & & \text { Average }+/-1 \Leftrightarrow & 1.01+/-0.20 \\ \text { D-359B } & 6.0 & 11.58 & 6.0 & 0.52 \\ \text { D-359C } & 7.5 & 12.02 & 7.0 & 0.58 \\ \text { D-359G } & 5.7 & 11.51 & 5.0 & 0.43 \\ \text { D-359H } & 6.5 & 11.74 & 5.0 & 0.43 \\ \text { D-359L } & 5.6 & 11.48 & 5.5 & 0.48 \\ \text { D-359M } & 7.5 & 12.02 & 7.5 & 0.62 \\ & & & \text { Average }+/-1 \Leftrightarrow & 0.51+/-0.08\end{array}$

Cylinders: $58 \mathrm{~mm}$ o.d.; $50 \mathrm{~mm}$ length; Assumed uniform effective surface area during bonding/sealing along edge. 
thickness; closer wrap positioning etc.), or possibly the extent of "sealing" which was added along the edges of each cylinder to provide an adequate test sealing surface were responsible for The gas flow resistance variations which led to what appeared to be a non-homogeneous filter body.

In an attempt to demonstrate particle collection efficiency, dust was delivered to each of the twelve cylindrical samples at room temperature for a period of 3 minutes. Both the clean inner surface appearance, as well as the absence of detectable fines in the off-gas stream indicated excellent particle collection efficiency of the PRD-66 matrix (Figure 2). When a particle challenged cylinder from the D-358 and D-359 series was fast fractured, fines were evident below the outer membrane-coated surface. As shown in Figure 3, the depth of fines penetration into the $6 \mathrm{~mm}$ filter wall varied from 1 to $3 \mathrm{~mm}$ indicating that the PRD-66 matrix had bulk rather than barrier filtration characteristics. Examination of the fast fractured surface indicated that the fines did not permeate across the entire $6 \mathrm{~mm}$ filter wall during the 3 minute dust exposure. Continued dust exposure testing would be needed to demonstrate the extent of fines penetration and/or plugging which may result during extended process operation.

\section{High Temperature, High Pressure Simulated PFBC Testing (Task 3.3)}

Three full length filters were subjected to high temperature, high pressure (HTHP) testing in Westinghouse's pressurized fluidized-bed combustion (PFBC) simulator in Pittsburgh, PA. These included candle filters D328 (improved, lower flow resistance dual membrane candles with a strengthened flange), D338 (standard membrane candles with a strengthened flange), and D325 (improved, lower flow resistance outer surface membrane candles with a strengthened flange). All three filter elements were mounted in the HTHP test facility, and the system was brought to temperature $\left(1550^{\circ} \mathrm{F}\right)$, and maintained at steady state conditions for two hours of operation with dust feed. After cool-down of the unit, areas along the outer surface of candle filter D328 and D325 were seen to have spalled off (Figure 4 ), while the standard outer surface membrane along candle filter D338 remained intact. The standard D338 membrane had typically been used at Tidd during the 1705 hour, Test Segment 4, and 1110 hour, Test Segment 5 campaigns. The failed membrane areas along D328 and D325 typically extended 1-2 inches, running parallel with the outer membrane winding pattern, and for 3-4 filament winding turns. Removal of the subsurface diamond pattern support structure was not evident (i.e., absence of initiation/propagation of "divoting"). Further development was recommended by Westinghouse to manufacture low gas flow resistance filter elements which maintained the integrity of the outer surface membrane.

\section{Modified Filter Membrane Evaluation (Task 3.1)}

Manufacturing modifications were undertaken to improve the bonding and integrity of the outer surface membrane of the PRD-66 candle, while maintaining the Westinghouse gas flow resistance criteria for as-manufactured filter elements. On October 16, 1996, two, 2 inch, PRD66 filter sections were received at Westinghouse. These were identified as:

- PRD-66 Combination membrane filter sample (492-5D)

- PRD-66 Particulate membrane filter sample (490-C).

Figure 5 illustrates the general appearance of both production configurations. The combination membrane consisted of: 


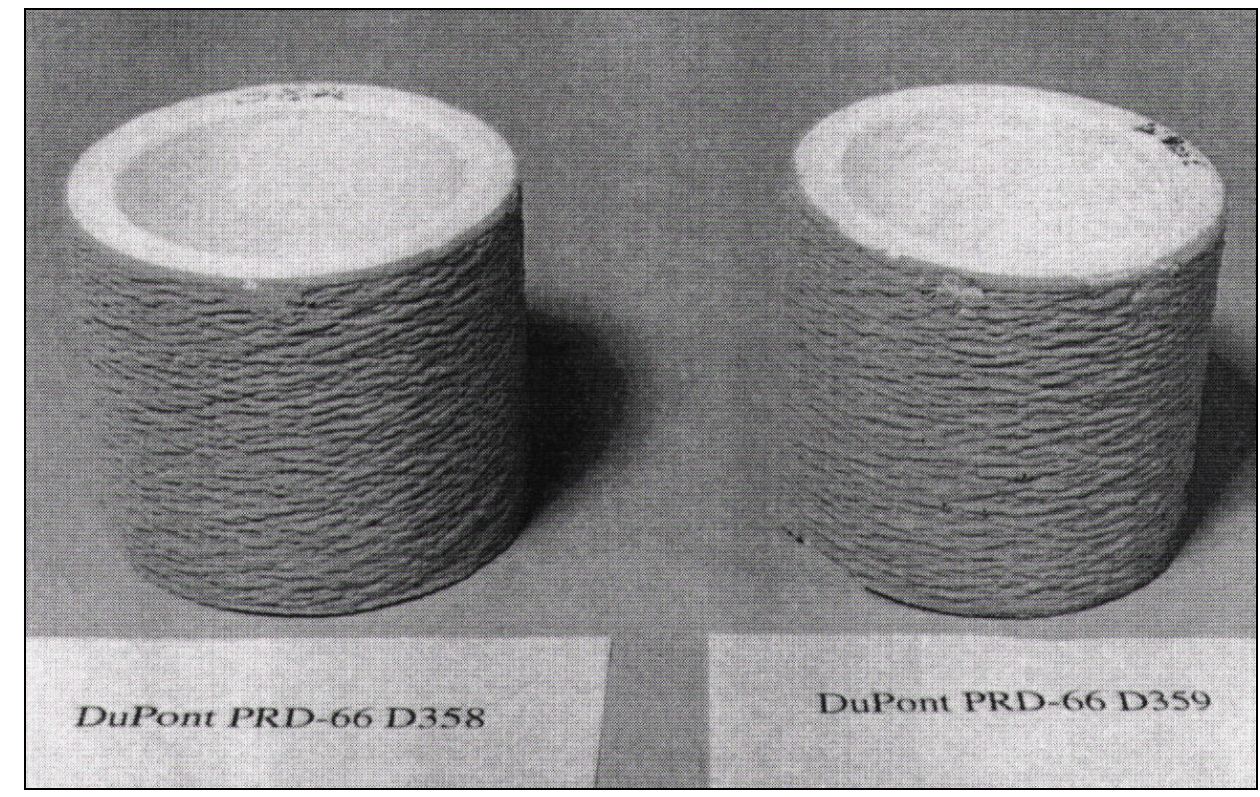

Figure 2 - DuPont PRD-66 filter matrices after room temperature particle collection and gas flow resistance testing. 

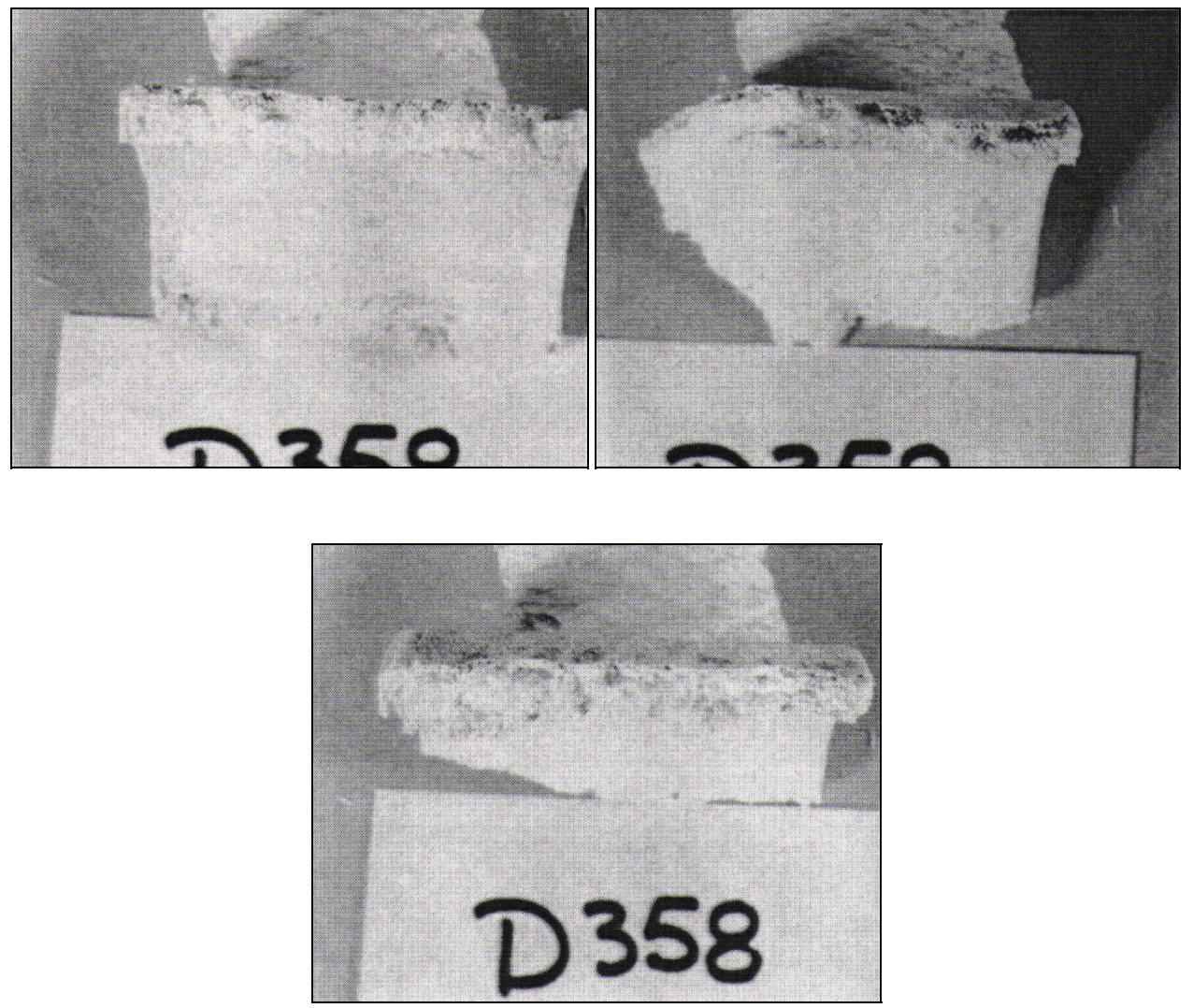

Figure $3 a-$ Fresh fractured surface of the particle challenged D-358 filter matrix. 

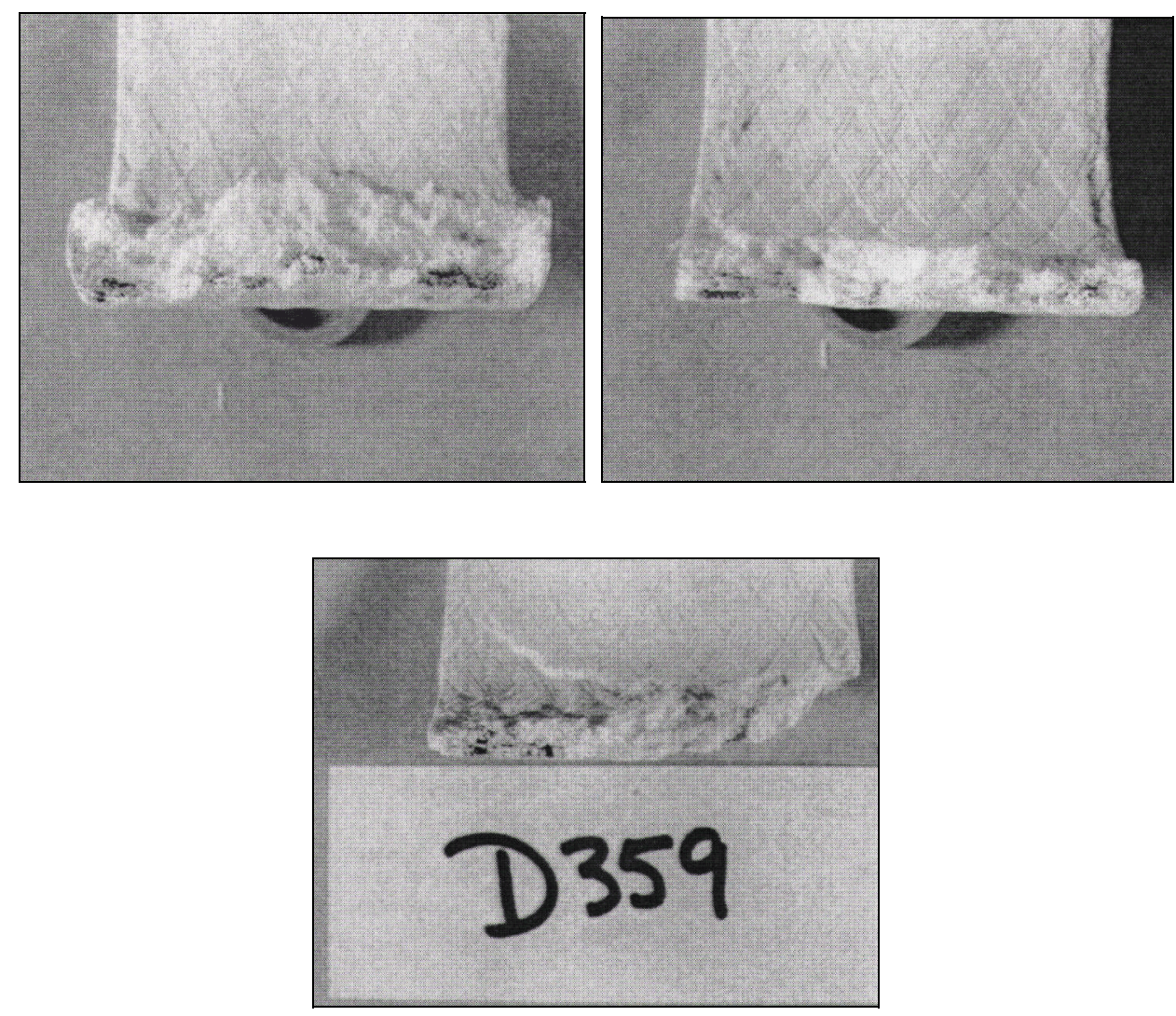

Figure $3 b$-- Fresh fractured surface of the particle challenged D-359 filter matrix. 

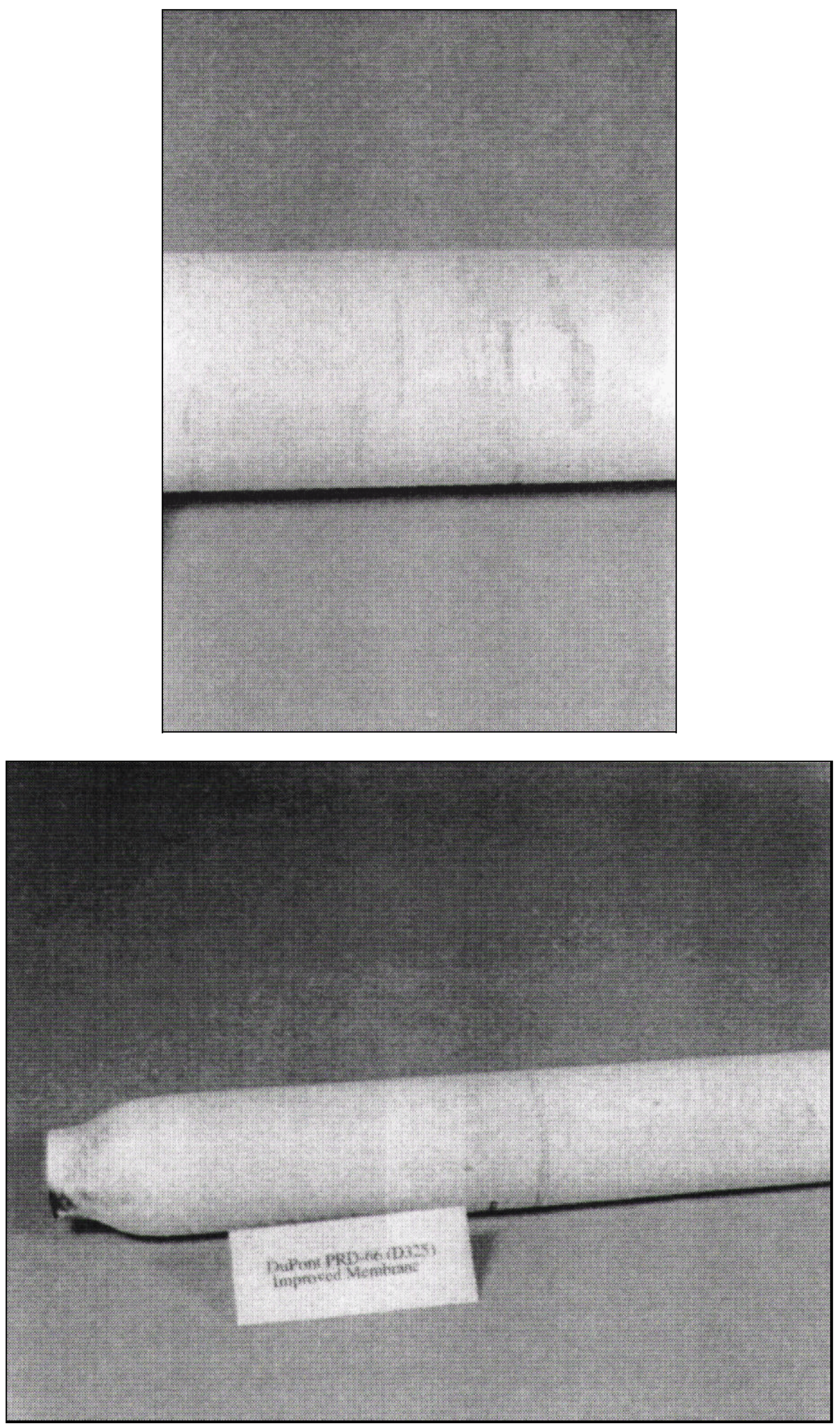

Figure 4a - HTHP-tested DuPont PRD-66 candle filter (Improved o.d. membrane; Strengthened flange). 


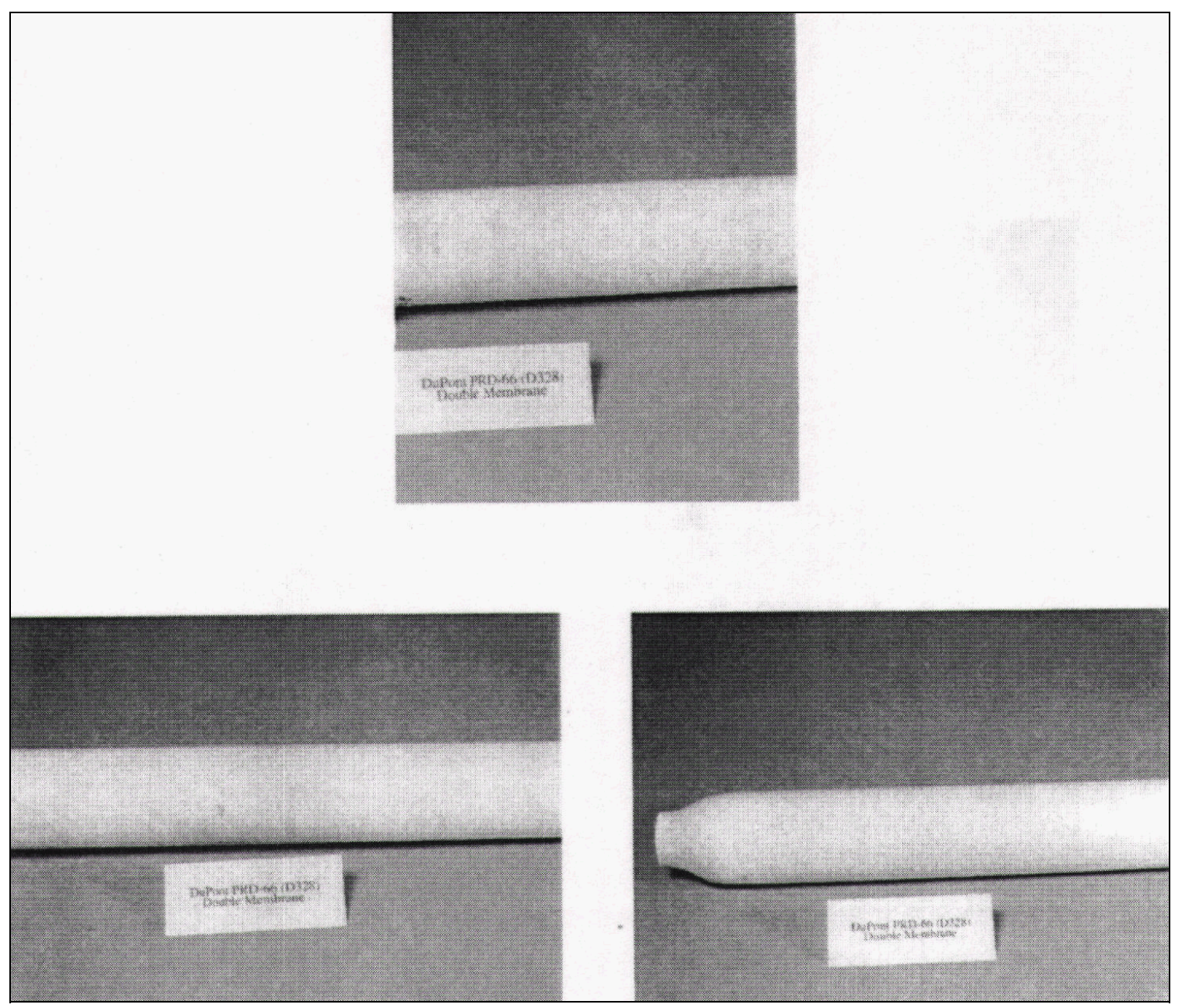

Figure 4b - HTHP-tested DuPont PRD-66 candle filter (Improved dual membrane; Strengthened flange). 


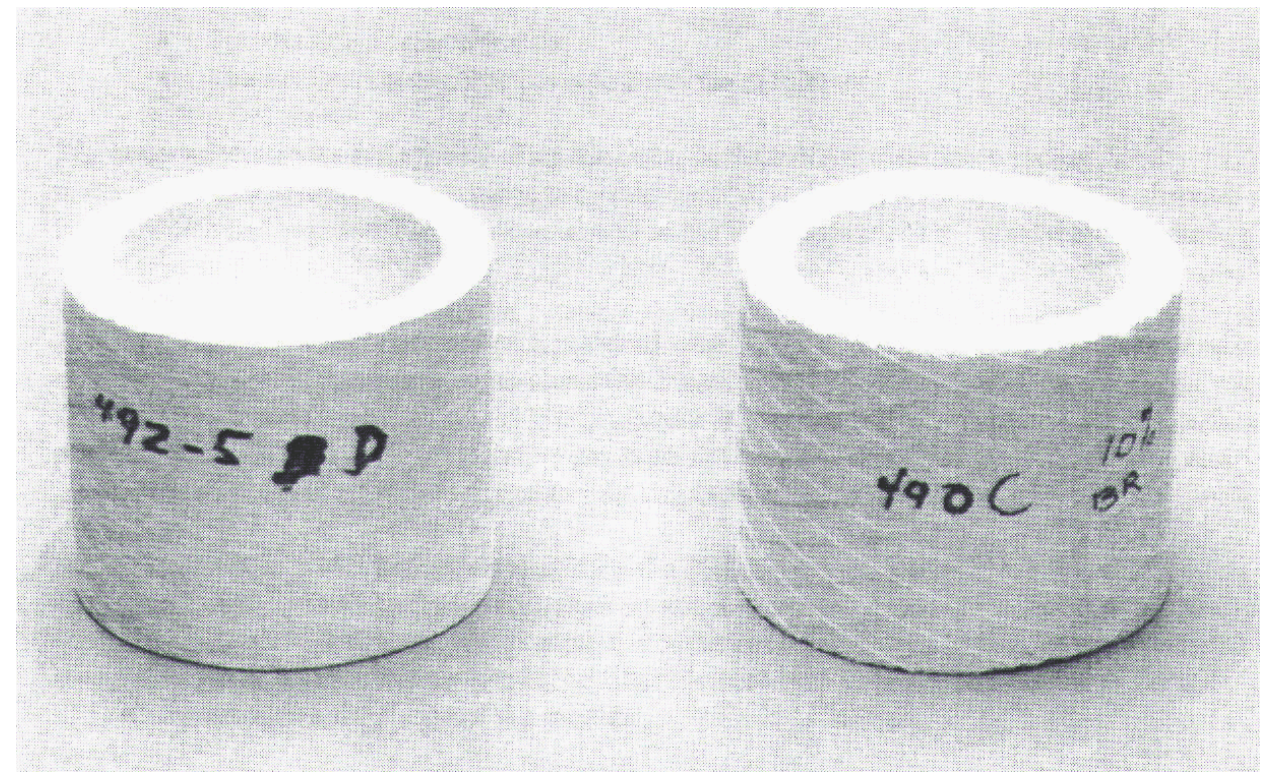

Figure 5 - PRD-66 combination membrane and particulate membrane filter concepts. 
- The prior diamond winding pattern which served as the bulk or support matrix

- An additional external hoop winding which formed a smooth surface outer membrane

- The application of an additional particulate slurry infiltration which was expected to reduce the gaps between the outer hoop winding, resulting in the formation of the combined hoop wrap and particulate membrane.

In contrast the particulate membrane filter concept consisted of:

- The diamond support matrix

- The infiltration of particulates to form the membrane.

The hoop winding was not applied along the outer surface of the diamond winding. Both matrices were developed in an attempt to circumvent "divoting" and subsequent filter element failure which had previously been experienced in the Westinghouse APF system at Tidd during Test Segment 5.

Initially 8-inch sections of each material were shipped to Westinghouse for consideration and/or evaluation. The uneven edges along the 2 -inch pieces which resulted from cutting of the filter sections at DLC were ground at Westinghouse in order to provide a smooth sealing surface prior to conduct of the room temperature gas flow resistance measurements. After testing and inspection, both samples were returned to DLC on October 21, 1996.

Table 2 provides comments regarding the PRD-66 combination membrane and particulate membrane filter concepts. Based on not only general appearance, but also the gas flow resistance measurements, Westinghouse recommended continued future development and manufacture of the combination membrane filter element with enhanced strengthening of the PRD-66 matrix along the flange of the candles.

Issues which remained to be addressed, however, included:

- Demonstrating the relative strength of both membrane filter concepts to identify if differences existed

- Demonstrating the relative load-to-failure for both membrane filter concepts to identify if differences existed

- Manufacturing of the filter sections and/or body with comparable o.d. dimensions. For the samples provided, the o.d. dimensions were not identical.

Based on the above information, Westinghouse supported production of the PRD-66 filter element with the combination membrane for use in future process simulation and/or field testing. Should the hoop wrap prove to be ineffective (i.e., bulk filtration vs. complete barrier filtration performance), additional modifications to the PRD-66 particulate membrane filter would be needed.

\footnotetext{
${ }^{3}$ Both the diamond winding pattern and external hoop were conceptually similar to what had previously been utilized to manufacture the filter elements installed at AEP.
} 
TABLE 2

COMPARISON OF PRD-66 FILTER MEMBRANE CONCEPTS

\begin{tabular}{|c|c|}
\hline $\begin{array}{c}\text { Combination Membrane } \\
\text { Hoop Wrap with Particle Infiltrate }\end{array}$ & Particulate Membrane \\
\hline $\begin{array}{c}\text { W-STC Gas Flow Resistance: } \\
0.5 \text { in-wg/fpm }\end{array}$ & $\begin{array}{c}\text { W-STC Gas Flow Resistance: } \\
1.07 \text { in-wg/fpm }\end{array}$ \\
\hline $\begin{array}{c}\text { DLC Gas Flow Resistance: } \\
0.9 \text { in-wg/fpm } \\
\end{array}$ & $\begin{array}{c}\text { DLC Gas Flow Resistance: } \\
1.2 \text { in-wg/fpm } \\
\end{array}$ \\
\hline $\begin{array}{l}\text { Gaps Between Hoop Wrap Winding Were } \\
\text { Evident. Potential Issues Include: } \\
\text {-- Penetration of Submicron Fines } \\
\text {-- Divot Formation Due to Thermal Expansion } \\
\text { of Penetrated Submicron Fines } \\
\text {-- Divoting Leading To Failure of The Element }\end{array}$ & $\begin{array}{l}\text { Particulate Infiltrate May Be More Evenly } \\
\text { Distributed Along The External Diamond Wrap Pattern. } \\
\text { If So, Then } \\
\text {-- Areas For Fines Penetration Into The Matrix } \\
\text { Which May Mitigate Or Reduce Divoting/Failure Of The } \\
\text { Filter Elements May Be Eliminated }\end{array}$ \\
\hline $\begin{array}{l}\text { Relatively Smooth Outer Surface } \\
\text {-- A Conditioned Ash Cake Layer May } \\
\text { Not Form Which May Lead To } \\
\text { Penetration Of Submicron Fines Into } \\
\text { The Interior Of The Filter Wall, } \\
\text { Potentially Causing Divoting and/or I } \\
\text { Failure Of The Element }\end{array}$ & $\begin{array}{l}\text { Stepped Surface Due To Diamond Patterns May } \\
\text {-- Be Potential Areas To Accumulate and/or Retain Ash } \\
\text { Fines } \\
\text {-- Lead To The Formation Of A Conditioned Ash Layer } \\
\text { Which Could Possess Bulk Filtration Characteristics } \\
\text {-- Pending Accumulation Of Fines Along The Diamond } \\
\text { Weave Edges, Localized Removal Of Fines May Not } \\
\text { Occur Leading To A High Pressure Drop Across The } \\
\text { Filter Element. }\end{array}$ \\
\hline \multicolumn{2}{|c|}{-- Minimal "Crumbling" Of Cut Surfaces In Contrast To Original Matrices } \\
\hline \multicolumn{2}{|c|}{$\begin{array}{c}\text {-- Along Cut Surfaces, Potential Delamination Areas Still Exist } \\
\text { Most Likely As A Result Of Bulk Substrate Winding Patterns. }\end{array}$} \\
\hline
\end{tabular}

* Differences between the Westinghouse and DuPont gas flow resistance measurements may be due to variations in the uniformity of the 2-inch vs. 8-inch sections, or alternately the measurement technique. 


\section{Qualification Testing for PCFBC Applications (Task 3.3)}

Eight, 1.5 m, PRD-66 candle filters were received from DuPont on March27, 1997. In the manufacturing process, either a coarse or medium grade hoop wrapped membrane was applied to the outer surface of the filter elements. The results of the room temperature gas flow resistance measurements of the eight, as-manufactured, $1.5 \mathrm{~m}$, candle filters are shown in Figures 6 and 7. Both sets of filter elements met the Westinghouse gas flow resistance tolerance of $<1 \mathrm{in}-\mathrm{wg} / \mathrm{fpm}$ for as-manufactured candles.

During April 1997, one candle of each filter element type was subjected to high temperature, high pressure (HTHP), simulated pressurized fluidized-bed combustion (PFBC) testing in Westinghouse's test facility in Pittsburgh, PA. Testing included exposure of the PRD-66 candle filters with alternate monolithic and advanced fiber reinforced candle filter elements in order to support pressurized circulating fluidized-bed combustion (PCPBC) test initiatives in Karhula, Finland. The filter array was subjected to 120 hours of steady state operating conditions at temperatures of $1550^{\circ} \mathrm{F}$, and subsequently 2200 accelerated pulse cycling, and 12 mild thermal transients events.

Post-test inspection of the filter army indicated that both PFBC-exposed PRD-66 filter elements remained intact. As a result, both elements, and an unexposed filter of each element type were subsequently subjected to mechanical strength characterization, and x-ray diffraction and microstructural analyses. The results of these efforts are summarized in the following sections. ${ }^{4}$

Figure 8 provides photographs of the residual dust cake layer that remained along the outer surface of the qualification-tested filter elements. Due the manner in which the qualification test was performed, the thin dust cake layer was considered to reflect the conditioned layer that generally remains attached to the outer surface of the candle during field exposure. Post-test gas flow resistance measurements of the qualification-tested candles are provided in Figure 9. The coarse membrane-coated filter element initially had a lower pressure drop in comparison the medium membrane-coated filter element. After qualification testing, this relationship was retained

\section{Bulk Strength Analysis}

As shown in Table 3, the strength of the coarse and medium membrane qualification tested DLC PRD-66 candle filters tended to be greater than the strength of comparable asmanufactured filter elements. As previously demonstrated by Westinghouse, the bulk strength of the DLC PRD-66 matrix tended to increase during simulated or field exposure [2] This was considered to result from the bulk vs. barrier filtration characteristics of the material, whereby submicron and micron fines penetrated through the membrane of the PRD-66 filter element and become entrapped within the filter wall. Although divot formations along the outer membrane did not occur during the qualification test program, the potential may still exist during extended

\footnotetext{
${ }^{4}$ Sections of both the coarse and medium membrane-coated, qualification-tested, PRD-66 filter elements were also returned to DLC on June 20, 1997, for additional inspection and characterization.
} 


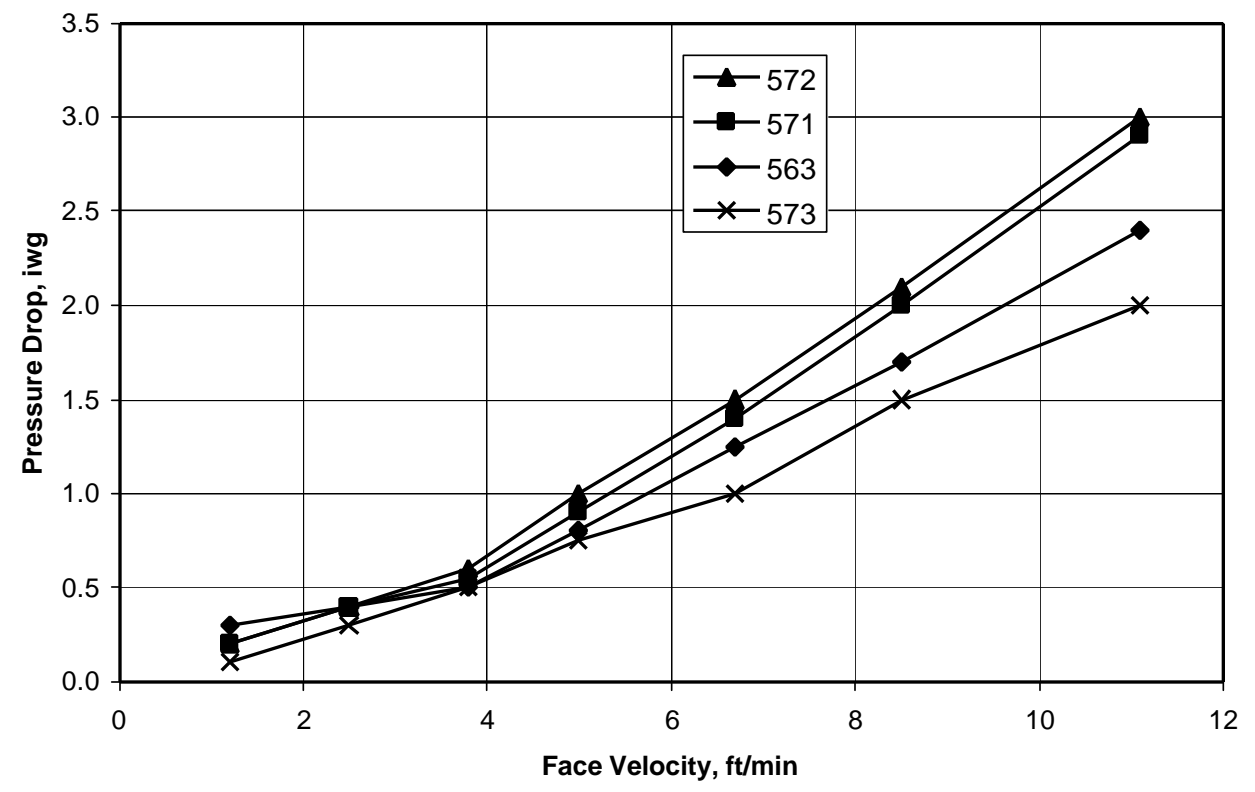

Figure 6 - Room temperature gas flow resistance measurements of the course membrane PRD-66 candle filters. 


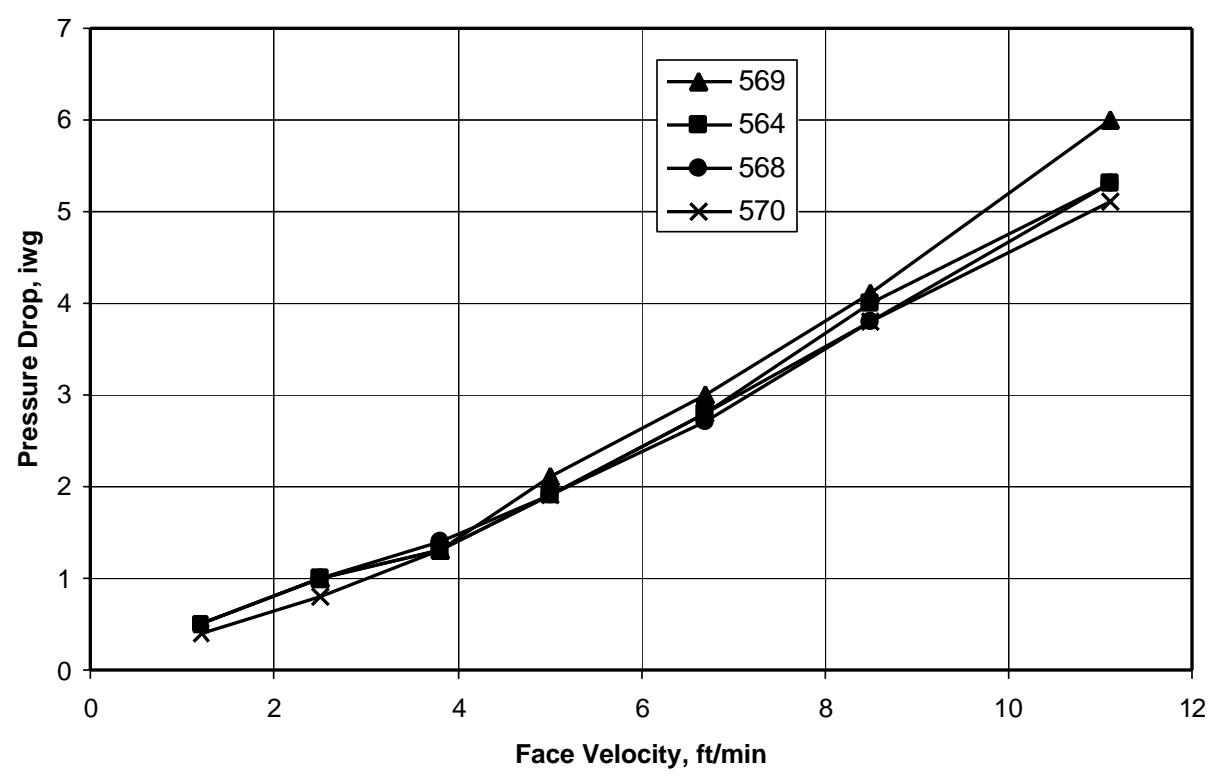

Figure 7 - Room temperature gas flow resistance measurements of the medium membrane PRD-66 candle filters. 


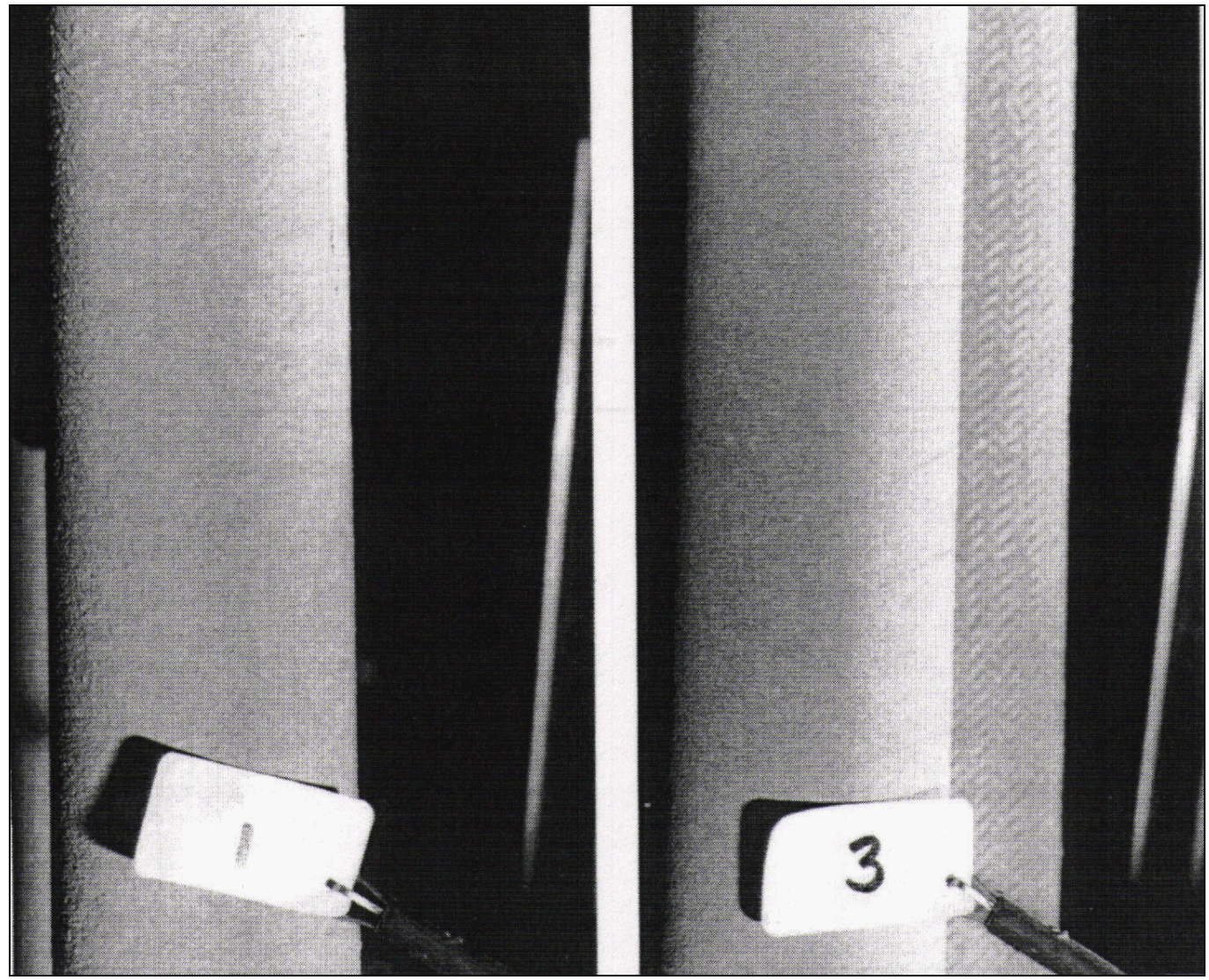

Figure 8 - Photograph illustrating the residual ash cake layer that remained along the outer surface of the PRD-66 candle filters after qualification testing that was conducted under simulated PFBC conditions. 

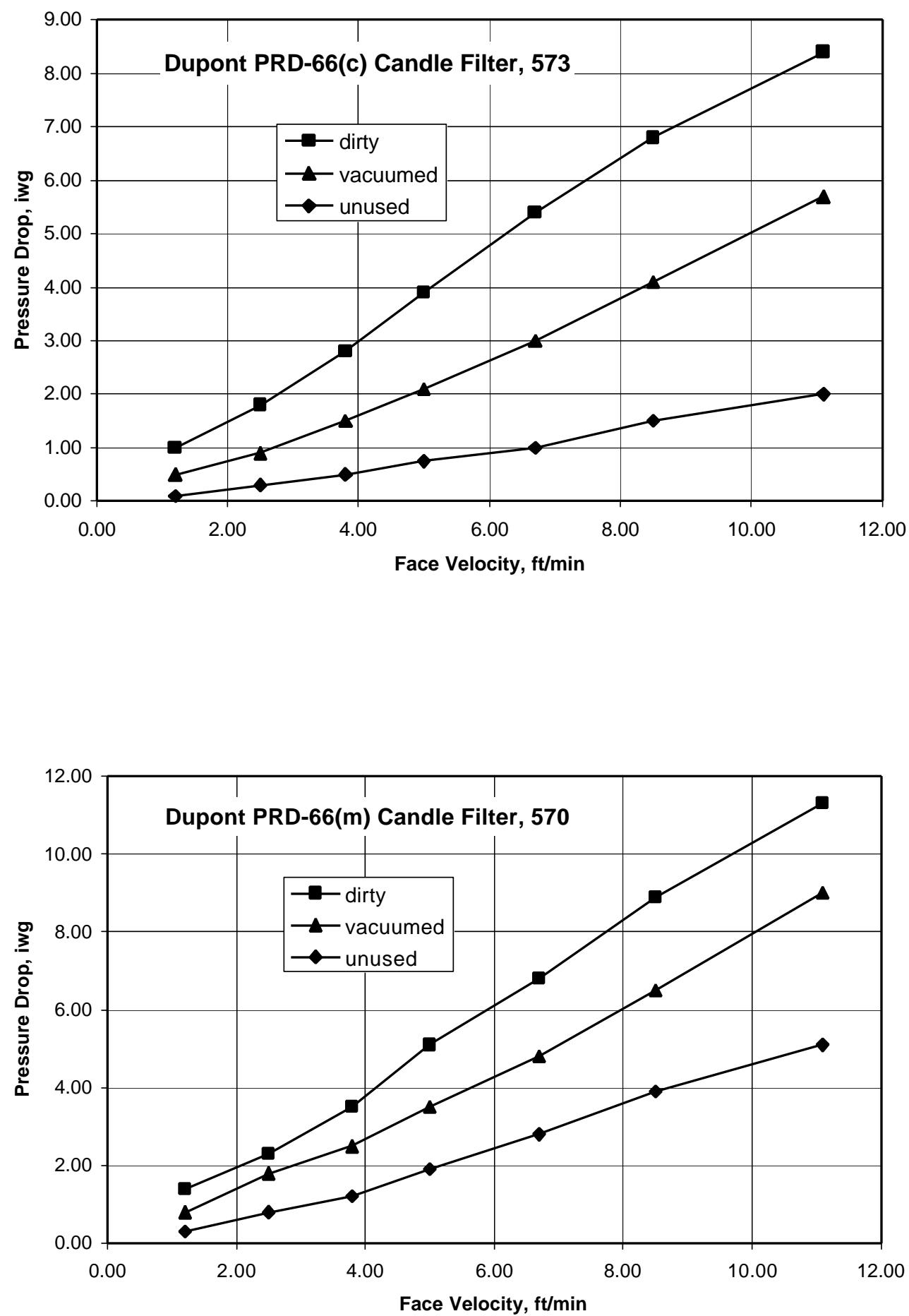
TABLE 3

\begin{tabular}{|c|c|c|c|c|c|}
\hline \multicolumn{6}{|c|}{$\begin{array}{c}\text { ROOM TEMPERATURE AND PROCESS STRENGTH OF THE } \\
\text { AS-MANUFACTURED AND QUALIFICATION-TESTED } \\
\text { DUPONT PRD-66 CANDLE FILTERS }\end{array}$} \\
\hline $\begin{array}{c}\text { Candle } \\
\text { Identification }\end{array}$ & \multirow[t]{2}{*}{ Status } & \multicolumn{2}{|c|}{$\begin{array}{c}\text { C-Ring Compressive Strength, } \\
\text { psi }\end{array}$} & \multicolumn{2}{|c|}{$\begin{array}{c}\text { C-Ring Tensile Strength, } \\
\text { psi }\end{array}$} \\
\hline Number & & $25-\operatorname{deg} C$ & 843-degC & $25-\operatorname{deg} C$ & 843-degC \\
\hline \multicolumn{6}{|c|}{ DuPont PRD-66 (Coarse Membrane) } \\
\hline D-563c & As-Manufactured & $955+/-62(9)$ & $962+/-92(8)$ & $809+/-154(9)$ & $1009+/-103(7)$ \\
\hline $\mathrm{D}-573 \mathrm{c}$ & Qualification Tested & $1214+/-67(9)$ & $1210+/-86(9)$ & $990+/-82(9)$ & $1195+/-166(9)$ \\
\hline \multicolumn{6}{|c|}{ DuPont PRD-66 (Medium Membrane) } \\
\hline D-564m & As-Manufactured & $990+/-130(9)$ & $883+/-79(9)$ & $846+/-105(9)$ & $918+/-104(9)$ \\
\hline $\mathrm{D}-570 \mathrm{~m}$ & Qualification Tested & $1021+/-127(9)$ & $1019+/-88(9)$ & $973+/-165(9)$ & $1193+/-149(8)$ \\
\hline
\end{tabular}

TABLE 4

\begin{tabular}{|c|c|c|c|c|c|}
\hline \multicolumn{6}{|c|}{$\begin{array}{c}\text { ULTIMATE LOAD APPLIED DURING STRENGTH CHARACTERIZATION } \\
\text { OF THE AS-MANUFACTURED AND QUALIFICATION-TESTED } \\
\text { DUPONT PRD-66 CANDLE FILTERS }\end{array}$} \\
\hline \multirow{2}{*}{$\begin{array}{l}\text { Candle } \\
\text { Identification } \\
\text { Number }\end{array}$} & \multirow[t]{2}{*}{ Status } & \multicolumn{2}{|c|}{$\begin{array}{l}\text { C-Ring Compressive } \\
\text { Load-to-Failure, psi }\end{array}$} & \multicolumn{2}{|c|}{$\begin{array}{c}\text { C-Ring Tensile } \\
\text { Load-to-Failure, psi }\end{array}$} \\
\hline & & 25-degC & 843-degC & 25-degC & 843-degC \\
\hline \multicolumn{6}{|c|}{ DuPont PRD-66 (Coarse Membrane) } \\
\hline \begin{tabular}{|c|}
$\mathrm{D}-563 \mathrm{c}$ \\
\end{tabular} & As-Manufactured & $8.2+/-0.5(9)$ & $8.2+/-0.9(8)$ & $5.2+/-1.1(9)$ & $6.7+/-0.7(7)$ \\
\hline D-573c & Qualification Tested & $10.3+/-0.6(9)$ & $10.3+/-0.6(9)$ & $6.4+/-1.2(9)$ & $7.6+/-1.0(9)$ \\
\hline \multicolumn{6}{|c|}{ DuPont PRD-66 (Medium Membrane) } \\
\hline D-564m & As-Manufactured & $8.0+/-0.9(9)$ & $7.3+/-0.6(9)$ & $5.2+/-0.6(9)$ & $5.7+/-0.6(9)$ \\
\hline D-570m & Qualification Tested & $8.3+/-1.0(9)$ & $8.3+/-0.8(9)$ & $6.1+/-0.9(9)$ & $7.4+/-0.8(8)$ \\
\hline
\end{tabular}


field operation, particularly if thermal expansion of the ash fines occurs within the filter wall during plant startup cycles [3], or hydration of the ash resulted during shutdown cycles.

In relation to alternate filter elements [4], the PRD-66 candle filter body was considered to be a moderately low load bearing matrix (Table 4). Additional material properties as burst strength, modulus, and Poisson's ratio, which were developed at Westinghouse are provided in Table 5.

\section{X-ray Diffraction Analysis}

An alternate explanation for increased strength conceivably is through crystallization of the matrix as a response of the material to the process gas chemistry and operating temperature. X-ray diffraction (XRD) analyses of the PRD-66 filter matrix identified the presence of $30 \%$ cordierite and $\sim 50 \% \alpha$-alumina, with mullite as a minor phase. The XRD patterns for the as-manufactured coarse and medium membrane matrices, and qualificationtested coarse and medium matrices appeared to be virtually identical. Since neither the qualification test exposure nor coarseness of the membrane affected phase assemblage, the concept of increased bulk strength as a result of fines infiltration was supported.

\section{Microstructural Characterization}

Sections of the PRD-66 filter matrices were removed from the qualification-tested filter elements, and were subjected to microstructural analyses via scanning electron microscopy energy disperse $x$-ray analyses (SEM/EDAX). Figures 10 and 11 illustrate the surface morphology of the coarse membrane-coated, qualification-tested, PRD-66 filter element. Random areas of ash were identified along the outer surface of the "cleaned" filter element (i.e., Area 1, Figure 10: relatively ash-free surface; Area 2, Figure 10: presence of fines). Although what appeared to be limited adherence of ash along the outer surface of the element, when viewed at higher magnification (Area 1, Figure 11), fines were readily seen to entrapped between adjacent, slurry deposited alumina-rich grains which formed the outer membrane surface. When viewed in cross-section, the fine graine membrane was seen to be adherently bonded to the underlying filament wound support fiber bundle structure (Figure 12). At higher magnification, ash fines were seen to be attached to individual grains contained within the membrane layer(Figure 13). Based on the microstructural analyses of the "cleaned", coarse membrane-coated, PRD-66 filter, the open porosity of the element was nearly completely retained after being subjected to simulated PFBC, qualification testing.

Similar microstructural analyses were conducted on the medium membrane-coated, qualification-tested, PRD-66 filter element. As shown in Figure 14 (i.e., Area 1), areas of ash were retained along the outer surface of the candle. When viewed at higher magnification, ash fines (Area 1, Photo 3, Figure 15; Photo 4, Figure 15) were seen to be contained between adjacent alumina-rich grains that were present in the outer membrane (Area 2, Photo 3, Figure 15). When fresh fractured, the cross-sectioned PRD-66 filter wall appeared to retain its relatively open porosity through both the membrane, as well as underlying filament wound structural support (Figure 16). At higher magnification (Figure 17), isolated ash fines were identified to adhere to either the outer surface of the alumina-rich membrane grains, or to the outer surface of the filament wound fiber bundles. 
TABLE 5

\begin{tabular}{|c|c|c|c|c|c|}
\hline \multicolumn{6}{|c|}{$\begin{array}{c}\text { MATERIAL PROPERTIES } \\
\text { OF THE AS-MANUFACTURED AND QUALIFICATION-TESTED } \\
\text { DUPONT PRD-66 CANDLE FILTERS }\end{array}$} \\
\hline $\begin{array}{c}\text { Candle } \\
\text { Identification } \\
\text { Number }\end{array}$ & Status & $\begin{array}{c}\text { Burst } \\
\text { Pressure, } \\
\text { psi }\end{array}$ & $\begin{array}{l}\text { Ultimate } \\
\text { Hoop } \\
\text { Stress, psi }\end{array}$ & $\begin{array}{l}\text { Modulus, } \\
\text { psi x } 10^{6}\end{array}$ & $\begin{array}{c}\text { Poisson's } \\
\text { Ratio }\end{array}$ \\
\hline \multicolumn{6}{|c|}{ DuPont PRD-66 (Coarse Membrane) } \\
\hline $\mathrm{D}-563 \mathrm{c}$ & As-Manufactured & 148 & 555 & 7.96 & 0.86 \\
\hline D-573c & Qualification Tested & 158 & 597 & 6.11 & 0.82 \\
\hline \multicolumn{6}{|c|}{ DuPont PRD-66 (Medium Membrane) } \\
\hline D-564m & As-Manufactured & 180 & 691 & 7.09 & $\overline{0.84}$ \\
\hline D-570m & Qualification Tested & 170 & 653 & 5.42 & 0.84 \\
\hline
\end{tabular}

TABLE 6

\begin{tabular}{|l|c|}
\hline \multicolumn{2}{|c|}{$\begin{array}{c}\text { Pressurized Circulating Fluidized-bed Combustion Testing at the } \\
\text { Foster Wheeler Test Facility in Karhula, Finland - TS2-97 }\end{array}$} \\
\hline Date & \begin{tabular}{c} 
September 4, 1997 - November 7, 1997 \\
\hline Number of Filter Elements Tested
\end{tabular} \\
\hline Filter Operating Temperature, deg.C & $700-750$ \\
\hline Filter Operating Pressure, bar & $9.5-11$ \\
\hline Coal Feed & Eastern Kentucky \\
\hline Sorbent & Florida Limestone \\
\hline Time, hrs & $581(6)^{*}, 342(1), 239(1)$ \\
\hline Face Velocity, cm/sec & $2.8-4.0$ \\
\hline Particle Load, ppmw & $6000-9000$ \\
\hline Particle Size, microns & $<1-150$ \\
\hline Thermal Excursions & None \\
\hline Number of Startup/Shutdown Cycles & 7 \\
\hline
\end{tabular}

* All elements remained intact. The number in parentheses indicates the number of elements exposed for the respective PCFBC operating hours. 


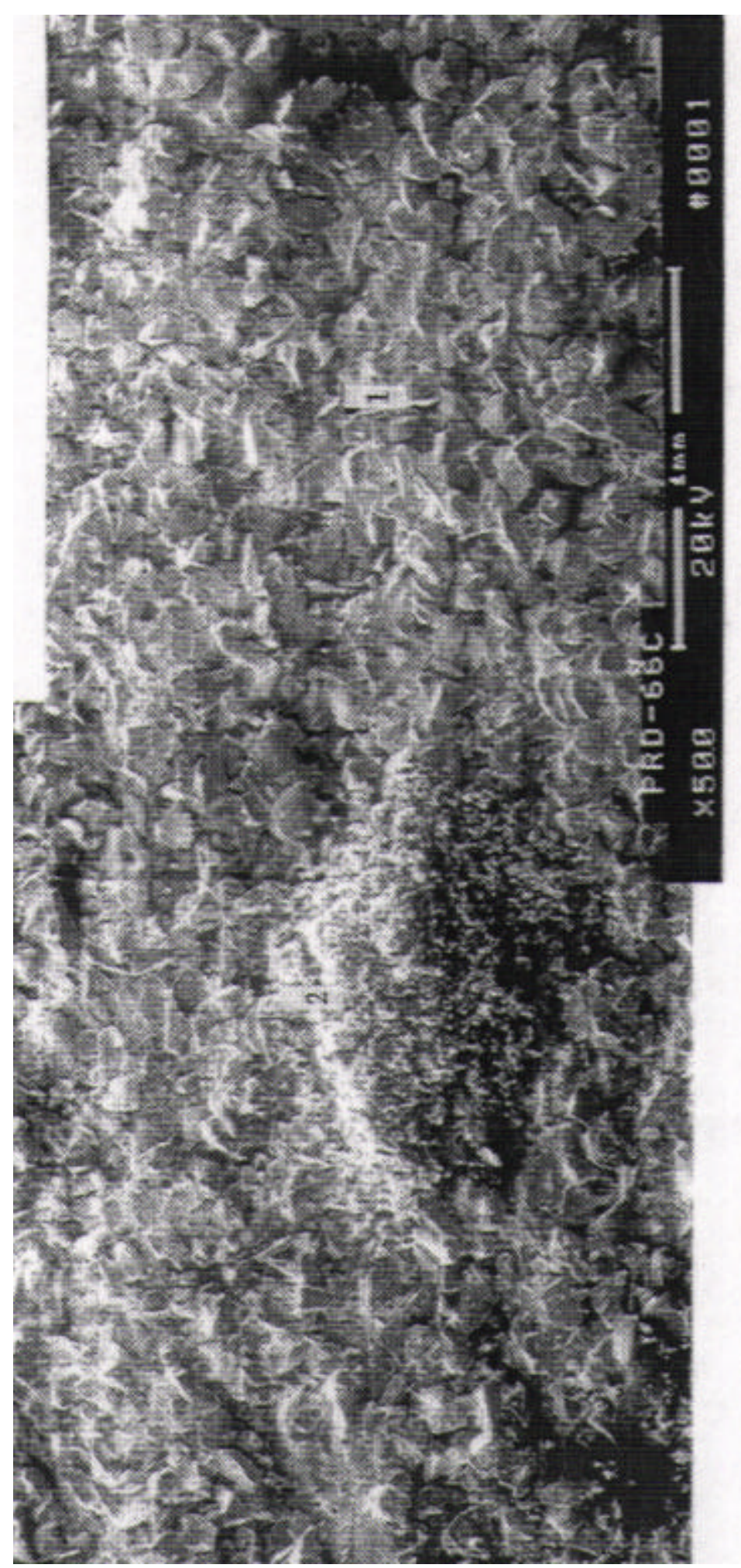

Figure 10 - Micrograph montage illustrating localized adherence of ash fines along the outer surface of the qualification-tested, coarse membrane-coated, PRD-66 filter element. 


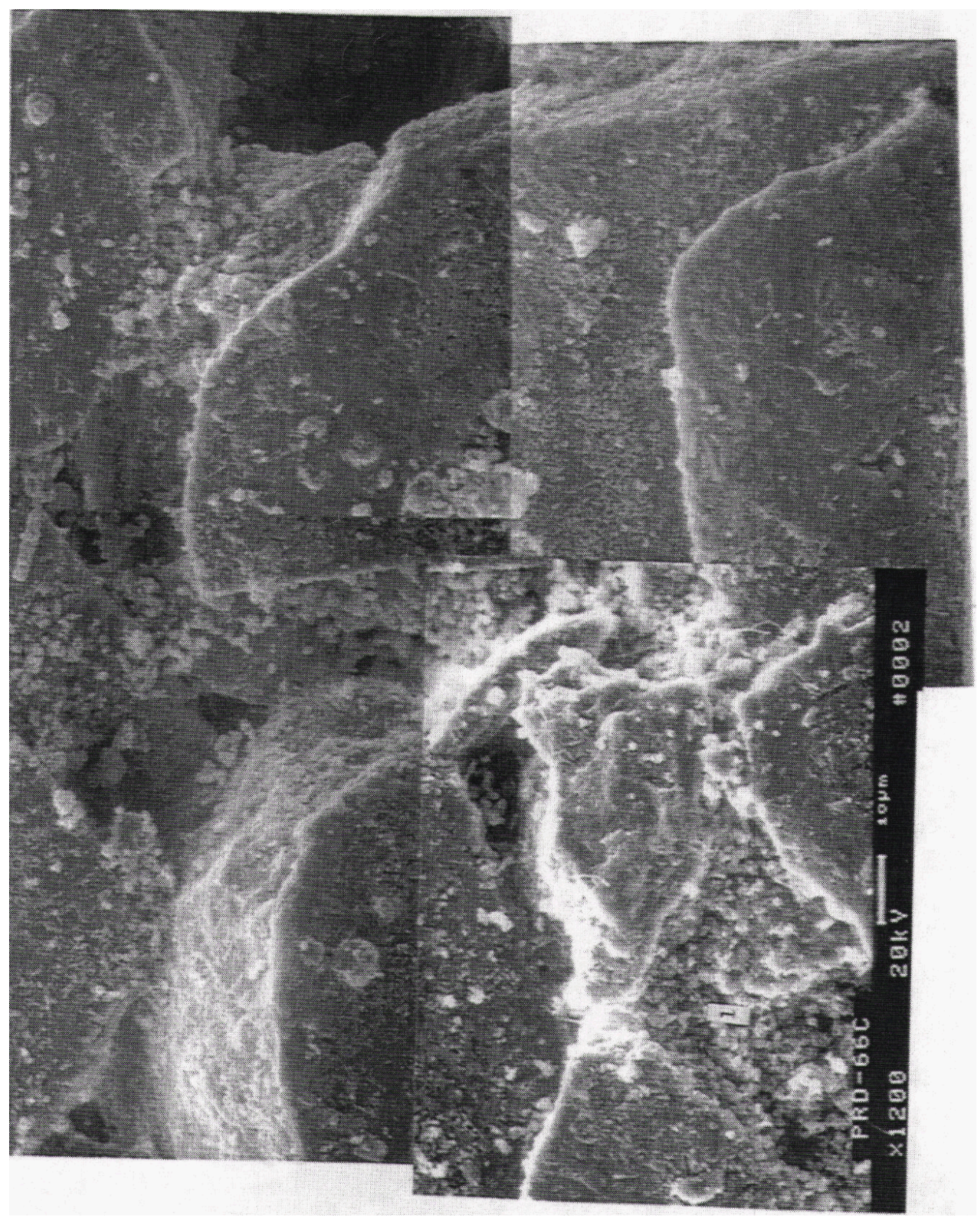

Figure 11 - Higher magnification micrograph montage illustrating the adherence of ash fines between adjacent alumina-rich grains present along the outer surface of the qualification-test, coarse membrane-coated, PRD-66 filter element. 


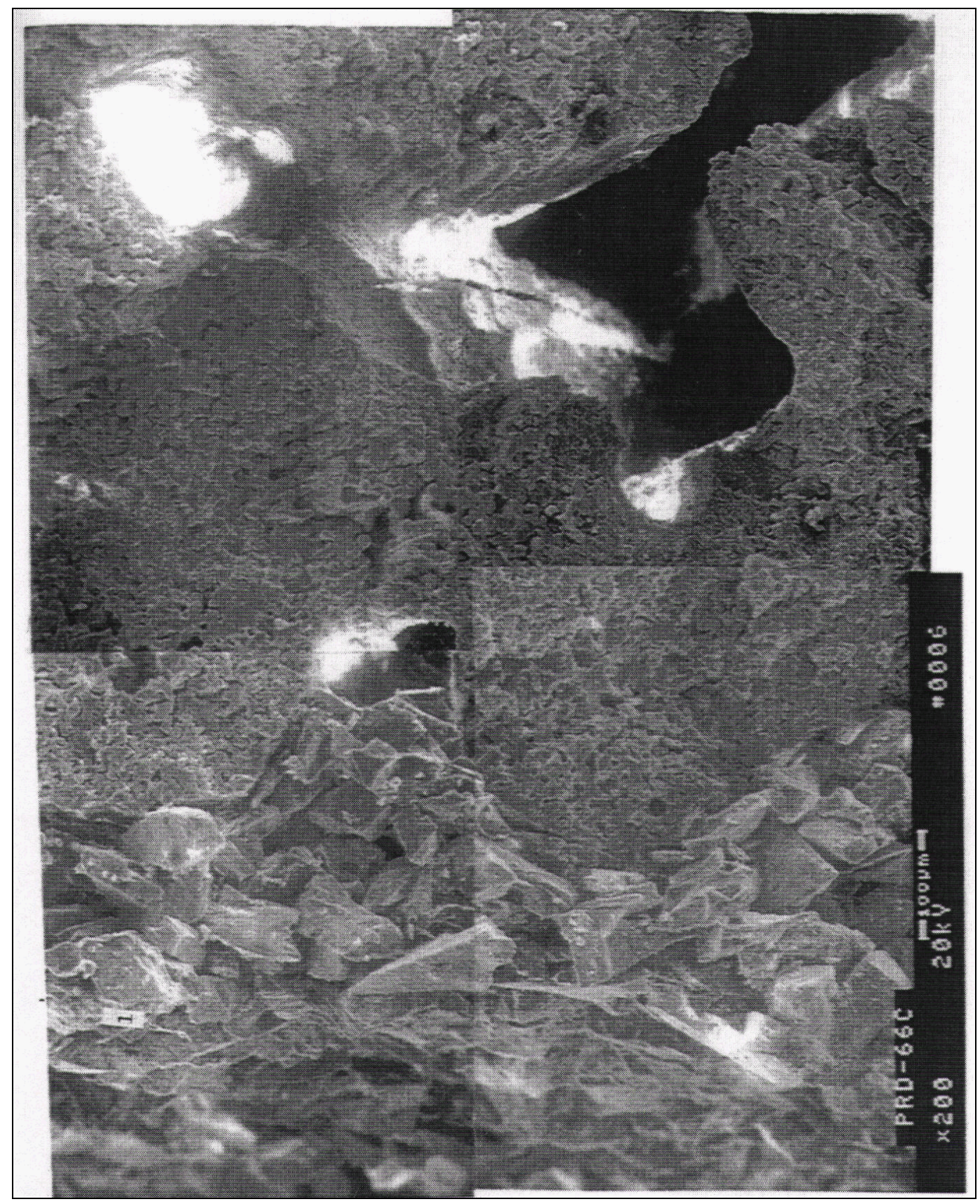

Figure 12 - Micrograph montage illustrating the morphology of the cross-sectioned filter wall of the qualification-test, coarse membrane-coated, PRD-66 filter element. 


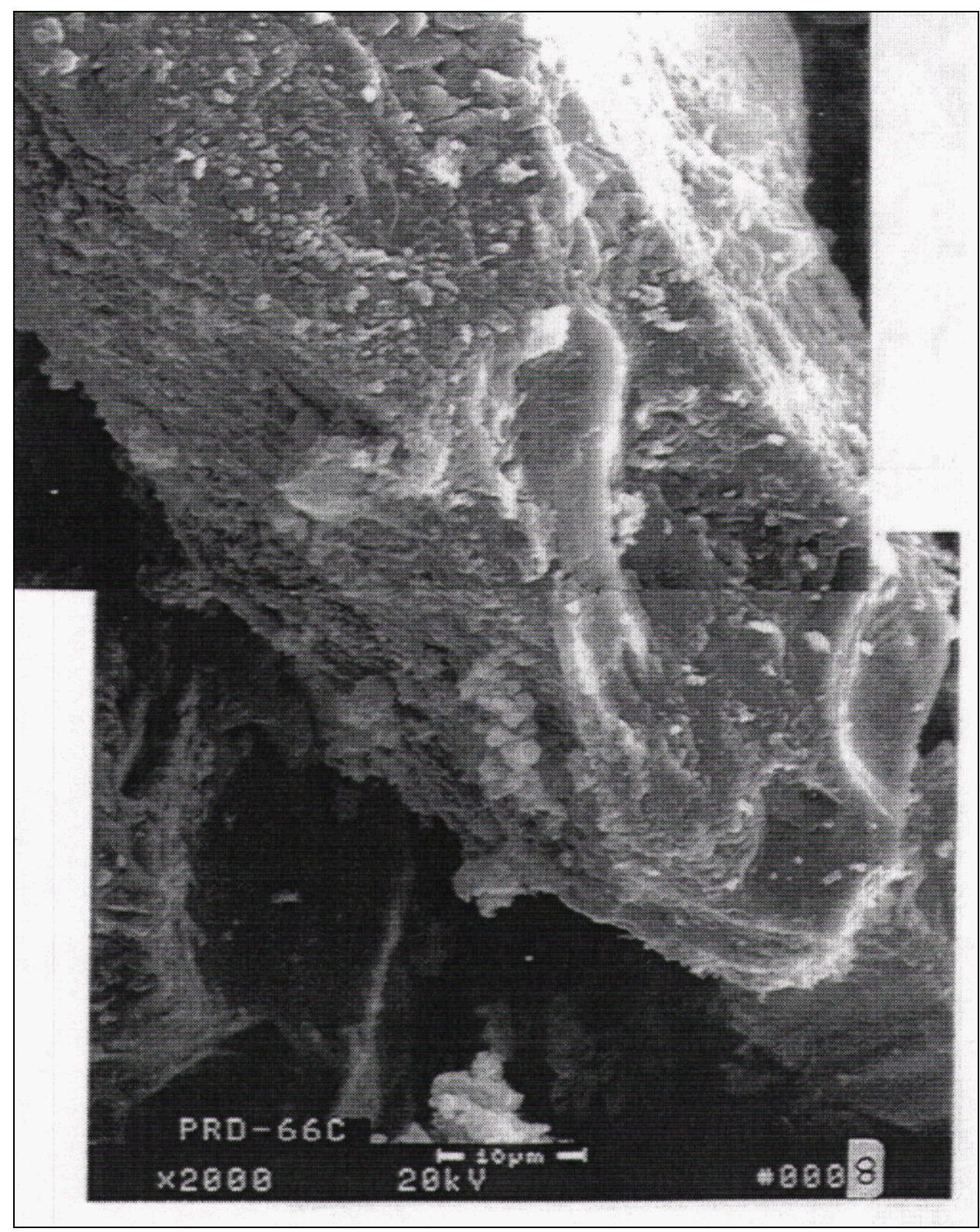

Figure 13 - Adherence of ash fines along the surface of the alumina-rich grains that were present within the outer surface membrane of the qualification-tested PRD-66 filter element. 


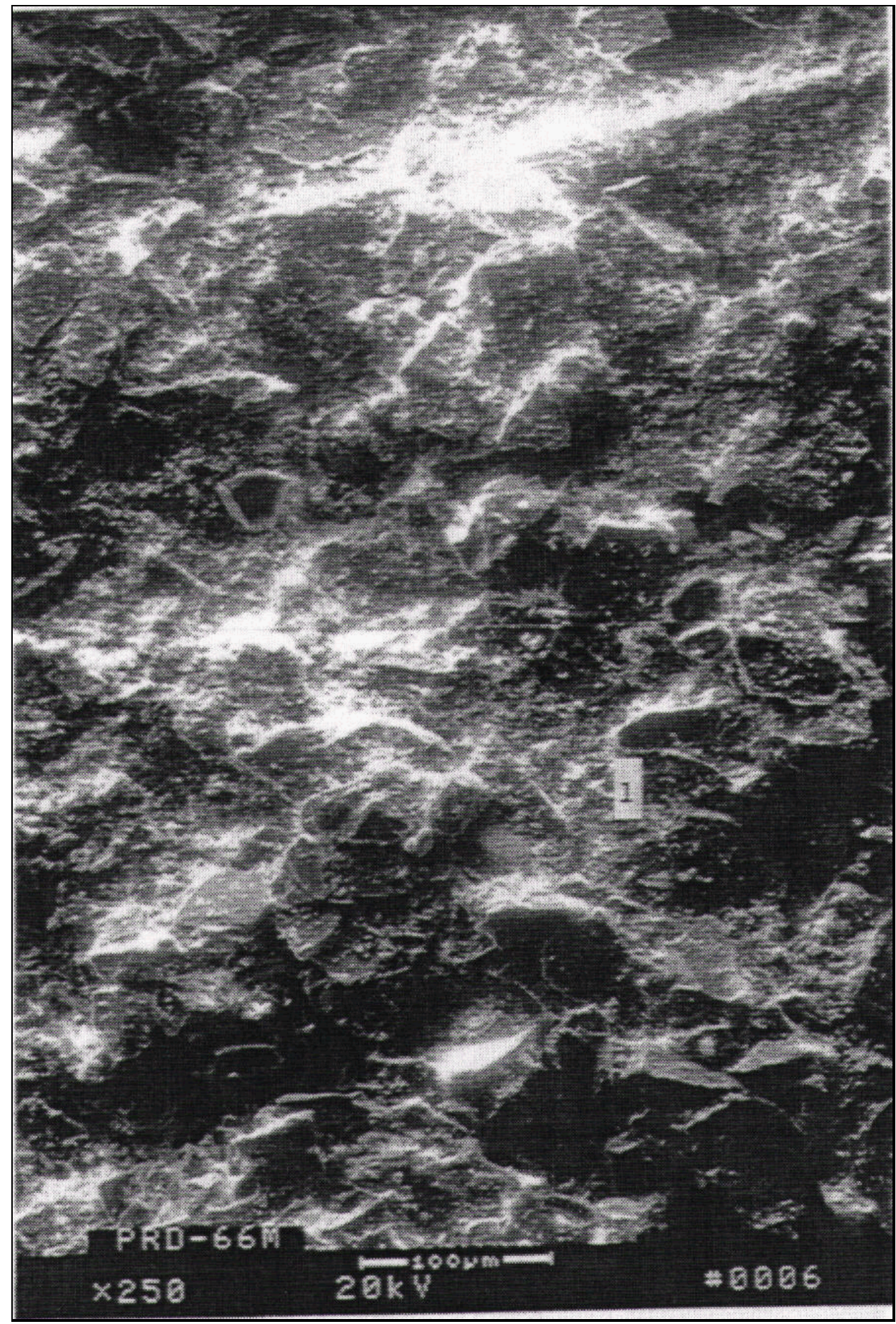

Figure 14 - Micrograph montage illustrating localized adherence of ash fines along the outer surface of the qualification-test, medium membrane-coated, PRD-66 filter element. 

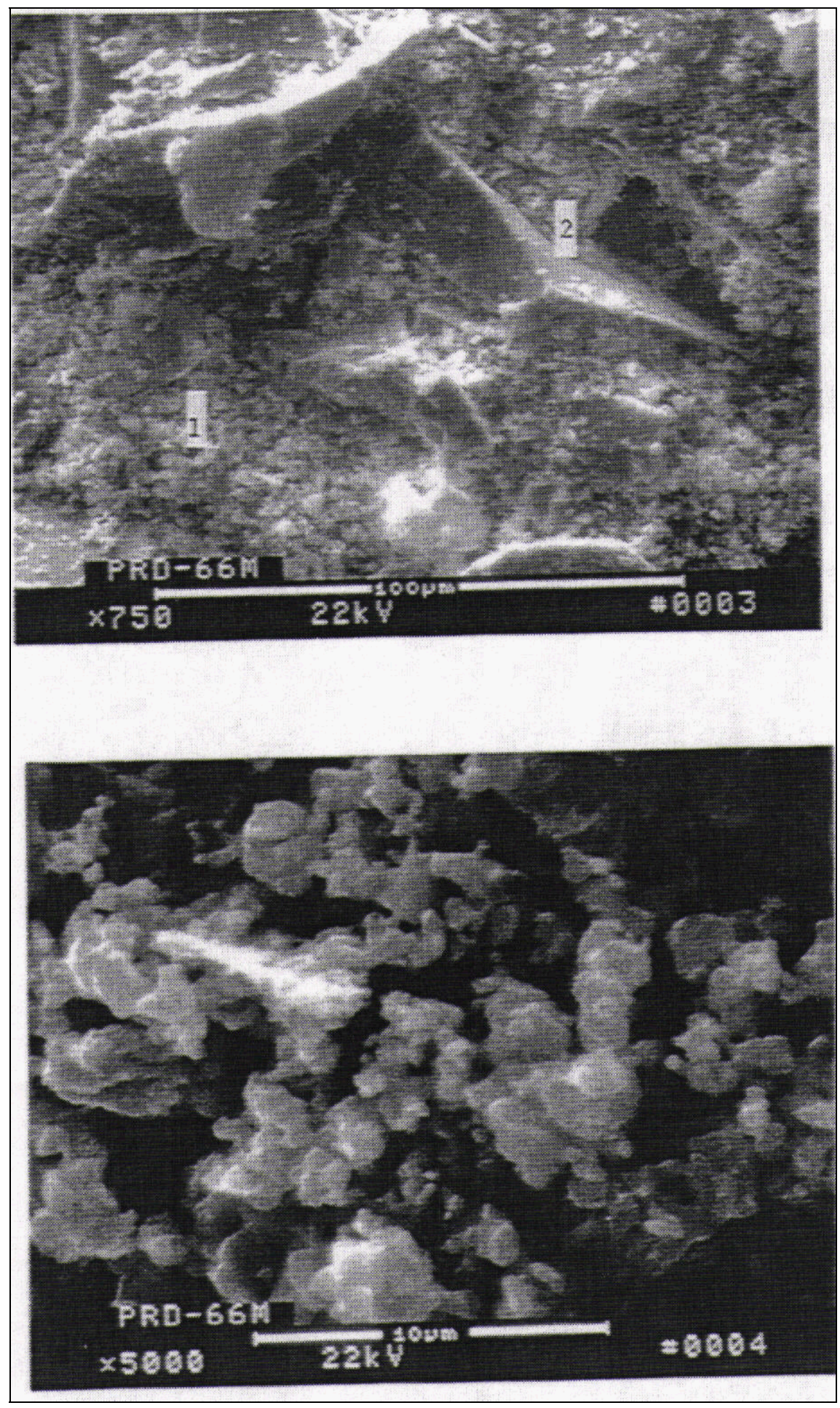

Figure 15 - Higher magnification micrographs illustrating the adherence of ash fines between adjacent alumina-rich grains present along the outer surface of the qualification-test, medium membrane-coated, PRD-66 filter element. The highly porous network of ash fines is shown in the lower micrograph. 


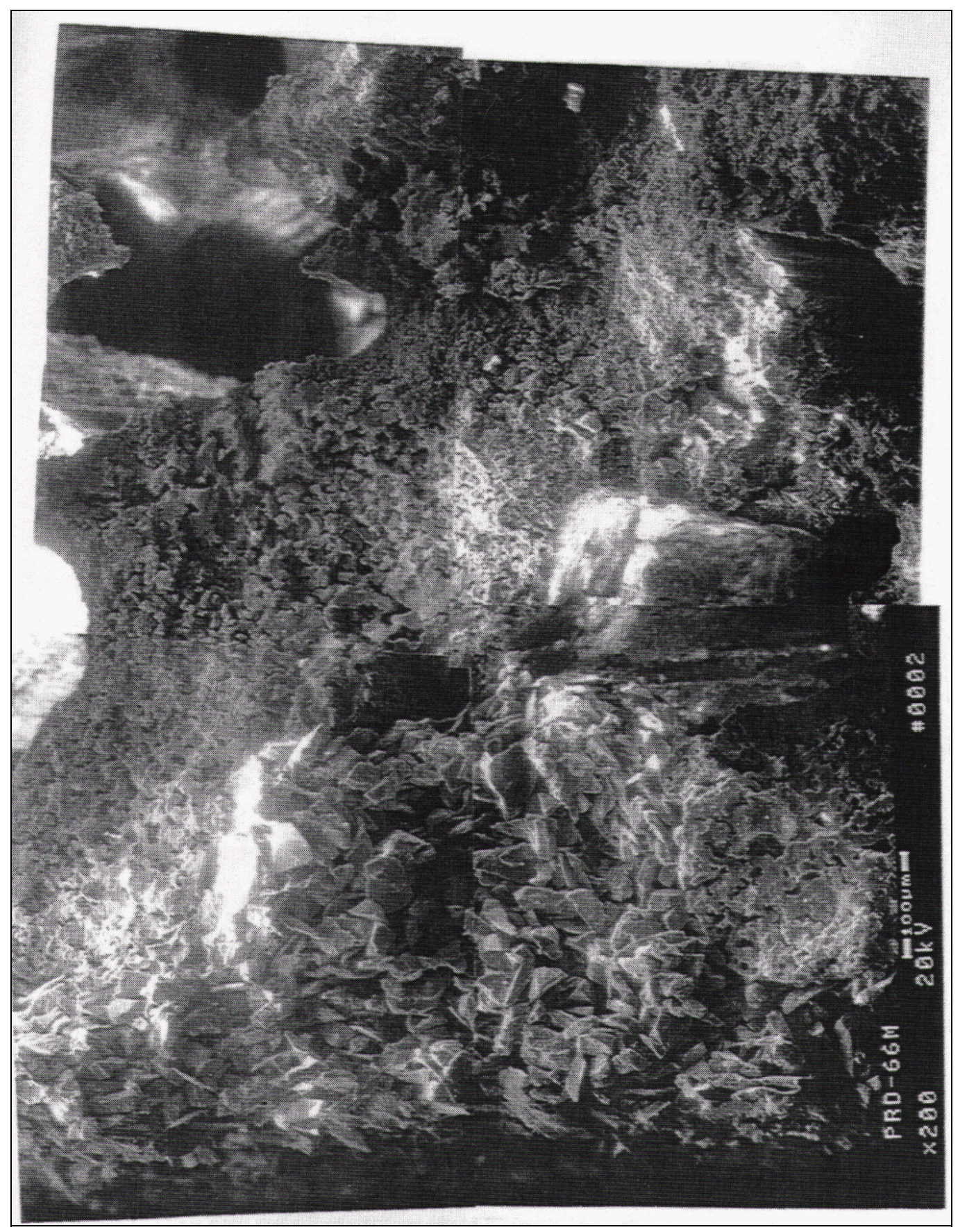

Figure 16 - Micrograph montage illustrating the morphology of the cross-sectioned filter wall of the qualification-test, medium membrane-coated, PRD-66 filter element. 


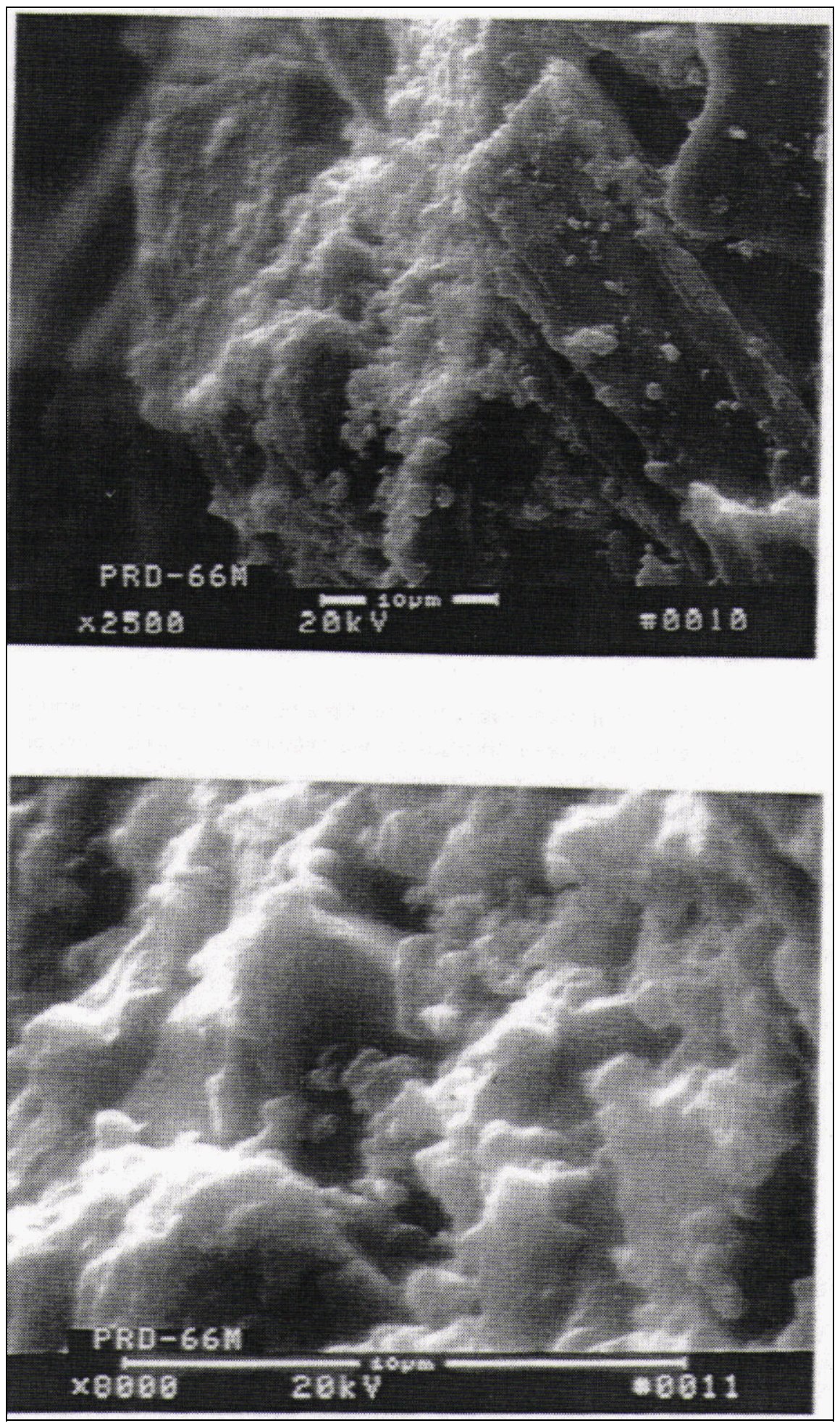

Figure 17 - High magnification micrographs illustrating the adherence of ash fines along the outer surface of the alumina-rich grains that were present within the membrane of the qualification-tested, medium membrane-coated, PRD-66 filter element. 


\section{Comment}

Limited penetration of ash fines into the membrane-coated filament wound filter matrix was identified for sections of the PRD-66 filter elements examined in this effort. Characterization of additional sections removed from the qualification-tested filter elements, and extended field operation (i.e., $>500-1000$ hours) are needed to confirm whether the DLC PRD-66 element performs as a barrier vs. bulk filter.

Based on the results of the qualification testing, both coarse and medium membranecoated filter elements were considered to be acceptable for use in Westinghouse's APF system at the Foster Wheeler PCFBC test facility in Karhula, Finland. In view of the gas flow resistance measurements for the as-manufactured candles, production of the coarse membrane-coated elements was selected as the filter type of choice for use at Karhula.

\section{PCFBC Candle Filter Testing}

Twelve, $1.5 \mathrm{~m}$, DuPont PRD-66 candle filters were manufactured with the coarse membrane coating, and shipped to Karhula at the end of July 1997. All twelve filter elements arrived intact, and were initially inspected, prior to consideration for inclusion within the Westinghouse APF. During inspection of the elements, the following comments were made:

- Generally all elements had a smooth outer surface finish

- Questions arose as to whether there would be an acceptable fit of the candle within the metal filter holder due to the extended length of the DLC hemispherical flange

- High intensity light source inserted along the i.d. of each filter element indicated general uniformity along the length of each candle

- On one or two of the elements, bands of denser areas of matrix were evident near the end caps

- On several elements, the intensity of the light appeared to be greater than along the body, possibly indicating a thinner area of the matrix

- If discontinuities existed, they were located at the bottom of the elements, near the end cap

- All end caps were generally uniform

- A section of the matrix ( $1-2 \mathrm{~mm}$ wide) was removed from the bottom end cap of one element during ultrasonic evaluation. This technique was modified to eliminate material removal during continued testing of the PRD-66 filter elements.

- Only one element had a slightly rougher outer membrane surface.

Seven DLC PRD-66 candles were installed in the bottom array of the Westinghouse APF, and were operated for a period of 342 to 581 hours (i.e., Test Segment 2: September 4, 1997 through November 7, 1997). Table 6 identifies the PCFBC operation conditions during conduct of this test campaign. At the conclusion of the test program, the filter vessel was slow cooled and inspected. All PRD-66 filter elements had remained intact during operation in the PCFBC environment. During removal from the filter array, one element failed at the base of the flange due to binding of the candle with ash in the filter holder mount, and the force required for disassembly. Divoting was not evident along the outer surface of the filter elements, implying that the integrity of the combination membrane had been retained during the first 581 hours of service life. Due to the relatively "soft" and fragile nature of the PRD-66 filter matrix, removal 
of the membrane (i.e., "nicks") occurred along several areas of the candles during disassembly of the elements from the filter array, as well as during cleaning and subsequent handling.

\section{Summary and Conclusions}

- The as-manufactured, outer membrane-coated DLC PRD-66 filter elements achieved the gas flow resistance specifications identified by Westinghouse.

- Continued production modifications have lead to the development and application of a coarse membrane coating along the hoop wrapped, outer surface of the filter elements. After 581 hours of exposure in the PCFBC environment, the integrity of the coarse membrane was retained.

- Further efforts are needed to address the barrier vs bulk filtration characteristics, of the PRD-66 filter element during long-term operation in PFBC, PCFBC, or gasification applications. This includes extensive microstructural analyses of the elements which have experienced greater than 500-1000 hours of field test exposure.

- Additional efforts remain to be focused on the development and production of the dual membrane, barrier candle filter; further strengthening of the flange; and the incorporation of a chip resistant outer surface.

\section{References}

I. M. A. Alvin, "Performance and Stability of Porous Ceramic Candle Filters during PFBC Operation," Materials at High Temperatures, 1997, 14 (3), 355-364.

2. M. A. Alvin, "Advanced Ceramic Materials for Use in High Temperature Particulate Removal Systems,"Ind. Eng. Chem. Res. 1996, 35, 3384-3398.

3. M. A. Alvin, T. E. Lippert, B. S. Diaz, E. B. Smeltzer, and R. E. Tressler, "Durability of Ceramic Filters," Proceedings of the Coal-Fired Power Systems '94-Advances in IGCC and PFBC Review Meeting, 545-571, DOE/METC-94/1008.

4. M. A. Alvin, T. B. Lippert, E. S. Diaz, B. B. Smeltzer, and G. J. Bruck, "Filter Component Assessment," Proceedings of the Advanced Coal Based Power and Environmental Systems 397 Contractor's Review Meeting, Pittsburgh, PA, July 22-24, 1997. 